\title{
Evaluation of the
}

\section{Miami-Dade Pedestrian Safety}

\section{Demonstration Project}
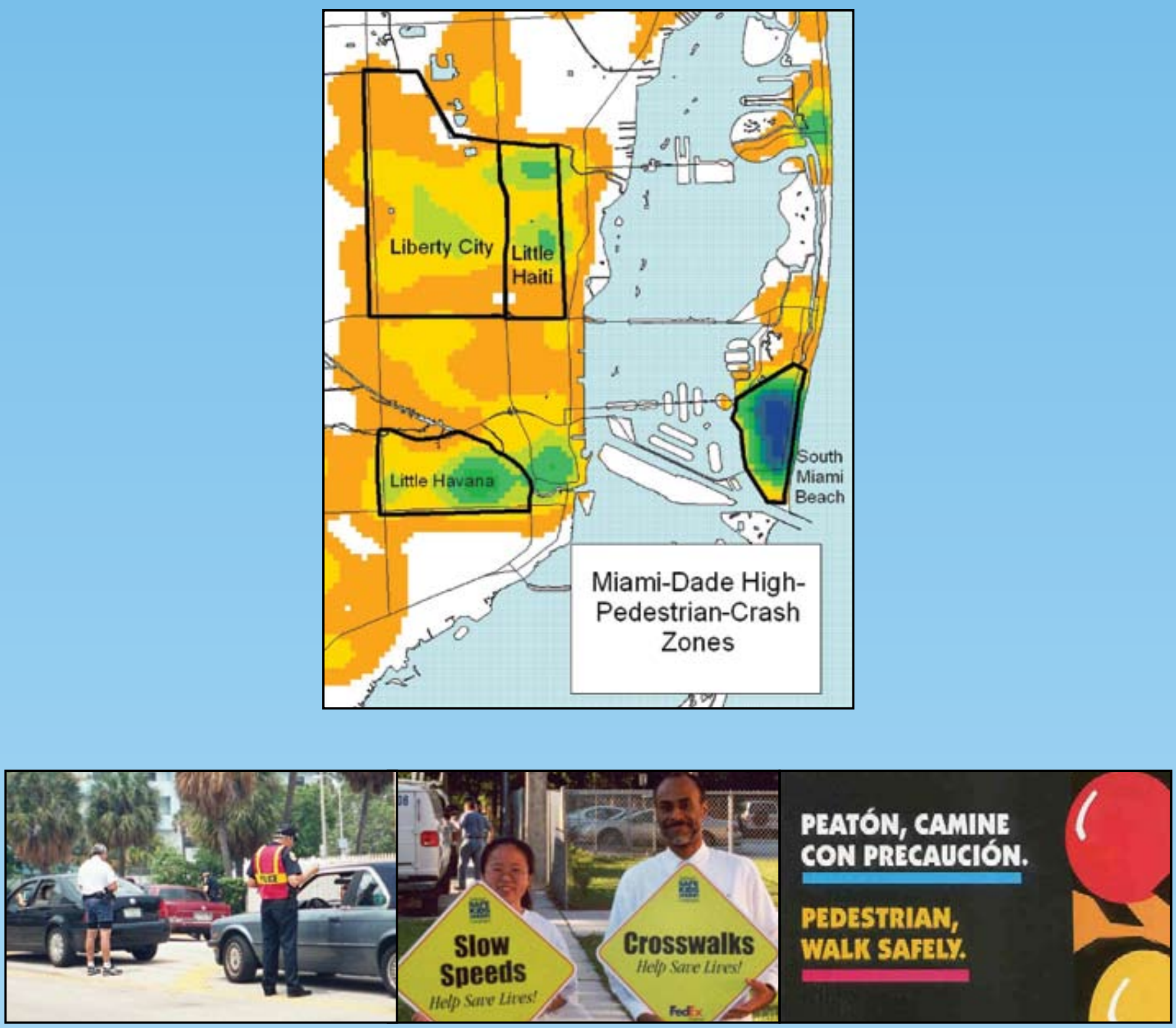
Technical Report Documentation Page

\begin{tabular}{|c|c|c|c|c|}
\hline 1. Report No. & \multicolumn{2}{|l|}{ 2. Government Accession No. } & \multicolumn{2}{|c|}{ 3. Recipient's Catalog No. } \\
\hline \multicolumn{3}{|c|}{$\begin{array}{l}\text { 4. Title and Subtitle } \\
\text { Evaluation of the Miami-Dade Pedestrian Safety Demonstration Project }\end{array}$} & \multicolumn{2}{|c|}{ 6. Performing Organization Code } \\
\hline \multirow{2}{*}{\multicolumn{3}{|c|}{$\begin{array}{l}\text { 9. Performing Organization Name and Address } \\
\text { University of North Carolina } \\
\text { Highway Safety Research Center } \\
730 \text { Martin Luther King Jr. Blvd, CB \#3430 } \\
\text { Chapel Hill, NC } 27599\end{array}$}} & \multicolumn{2}{|c|}{ 10. Work Unit No. (TRAIS) } \\
\hline & & & $\begin{array}{r}\text { 11. Contract or C } \\
\text { DT }\end{array}$ & \\
\hline \multirow{3}{*}{\multicolumn{3}{|c|}{$\begin{array}{l}\text { 12. Sponsoring Agency Name and Address } \\
\text { National Highway Traffic Safety Administration } \\
1200 \text { New Jersey Avenue SE. } \\
\text { Washington, DC } 20590\end{array}$}} & \multirow{2}{*}{\multicolumn{2}{|c|}{$\begin{array}{l}\text { 13. Type of Report and Period Covered } \\
\qquad \text { Final Report } \\
\text { October } 1998 \text { to September } 2007\end{array}$}} \\
\hline & & & & \\
\hline & & & \multicolumn{2}{|c|}{ 14. Sponsoring Agency Code } \\
\hline \multicolumn{5}{|c|}{$\begin{array}{l}\text { The contract manager for this report was Dr. Marvin M. Levy (NHTSA). Dunlap and Associates, Inc., of Stamford, } \\
\mathrm{CT} \text {, and the Miami-Dade Metropolitan Planning Association were subcontractors. Eric Rodgman contributed to the } \\
\text { data processing and analysis. Laura Sandt and Michael Daul contributed to the report design, layout, and editing } \\
\text { process. }\end{array}$} \\
\hline \multicolumn{5}{|c|}{$\begin{array}{l}\text { The purpose of this study was to identify and implement a comprehensive countermeasure program that could reduce } \\
\text { deaths and injuries among pedestrians in a large urban environment. Miami-Dade County, Florida, was selected as the } \\
\text { focus of this study. Using pedestrian crash data from } 1996 \text { to } 2001 \text {, four zones were identified within the county as } \\
\text { having abnormally high pedestrian-crash experiences. Based on locational crash characteristics, as well as pedestrian } \\
\text { (age, ethnicity) factors, a total of } 16 \text { different types of education, enforcement, and engineering treatments were } \\
\text { selected and targeted to reduce pedestrian crashes specifically in the four zones, and also countywide. }\end{array}$} \\
\hline \multicolumn{5}{|c|}{$\begin{array}{l}\text { A before-after study was used with three separate control groups to evaluate the effects of the combined pedestrian } \\
\text { safety program on pedestrian crashes. A three-year "after" period was used (2002-2004). Multivariate intervention } \\
\text { autoregressive integrated moving average (ARIMA) time series analysis was used, along with nonparametric (i.e., } \\
\text { Mann-Whitney U-tests) to test for statistically significant differences in pedestrian crash experiences. Results showed } \\
\text { that, at the peak of the program effects in } 2003 \text { and } 2004 \text {, the pedestrian safety program reduced countywide pedestrian } \\
\text { crash rates by between } 8.5 \% \text { and } 13.3 \% \text {, depending on which control group was used. This effect translates to } \\
\text { approximately } 180 \text { fewer crashes annually in Miami-Dade County, or } 360 \text { pedestrian crashes reduced in } 2003 \text { and } 2004 \\
\text { combined. Countywide, the greatest crash reductions were found among children and adult pedestrians as a result of } \\
\text { the program. Educational and other measures to reduce crashes involving older pedestrians showed no effect. A } \\
\text { number of lessons learned were identified for future implementation of such a program. }\end{array}$} \\
\hline \multicolumn{2}{|c|}{$\begin{array}{l}\text { 17. Key Words: } \\
\text { Pedestrian, safety, education, enforcement, engineering, } \\
\text { effectiveness, evaluation, countermeasure, comprehensive } \\
\text { safety program, crash analysis, local partnerships }\end{array}$} & \multicolumn{3}{|c|}{$\begin{array}{l}\text { 18. Distribution Statement } \\
\text { This report is free of charge from the NHTSA Web site } \\
\text { at www.nhtsa.dot.gov }\end{array}$} \\
\hline 19. Security Classif. (of this report) & \multicolumn{2}{|c|}{$\begin{array}{l}\text { 20. Security Classif. (of this page) } \\
\text { Unclassified }\end{array}$} & 21. No. of Pages & 22. Price \\
\hline
\end{tabular}

Form DOT F 1700.7 (8-72)

Reproduction of form and completed page is authorized 


\section{ACKNOWLEDGEMENTS}

The authors wish to acknowledge a number of people for their contribution to the overall study effort. These include:

- Cindy Magnole, Jackson Memorial Medical Center

- Sue Ignash, Florida Dept of Health

- Frances Gray, Miami-Dade County Health Department

- Carole Steans, Lehman Injury Research Center

- Frank Saavedra, Miami-Dade Fire Rescue

- Henry Oliver, Miami-Dade Police Department

- Armando Penedo, Miami-Dade Police Department

- Lewis Sayre, Florida Department of Transportation (FDOT)

- Chief Pat Kiel, City of West Miami Police Dept

- Vivian Villaamil, Miami-Dade County Public Schools

The authors also appreciate the efforts by Mimi Sutherland, Brian Hannigan (Miami Children's Hospital, chair of Miami-Dade Safe Kids Coalition), Dr. Gillian Hotz (University of Miami/Jackson Memorial Medical Center), Dr. Ron Van Houten (Western Michigan University), Dr. Ilir Bejleri (University of Florida), Dr. Ruth Steiner (University of Florida), and Pat Pieratte (Florida Department of Transportation). 


\section{TABLE OF CONTENTS}

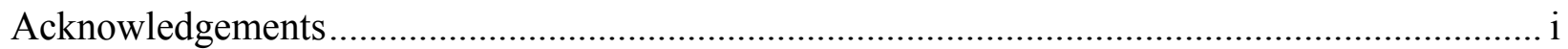

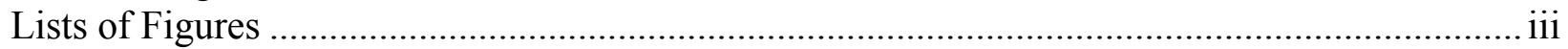

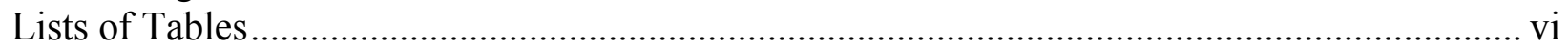

Technical Summary .................................................................................................

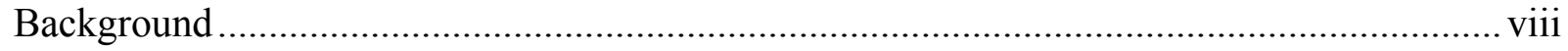

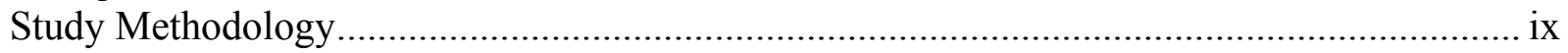

Description of Pedestrian Safety Treatments.....................................................................

Educational Countermeasures.......................................................................................

Enforcement Countermeasures .................................................................................. xiv

Engineering Countermeasures ……………………................................................. xiv

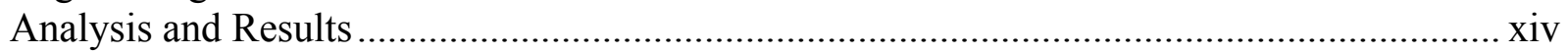

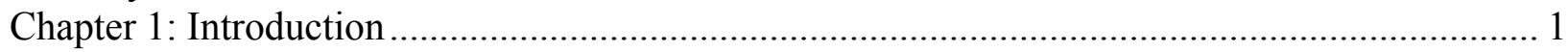

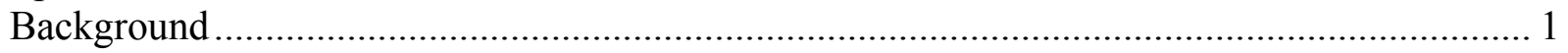

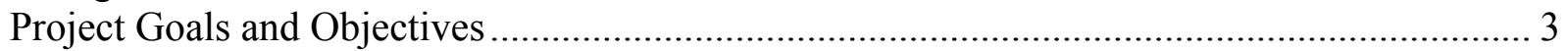

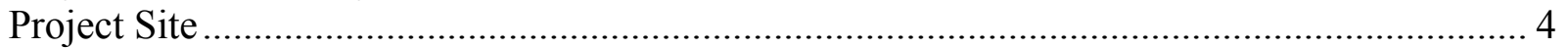

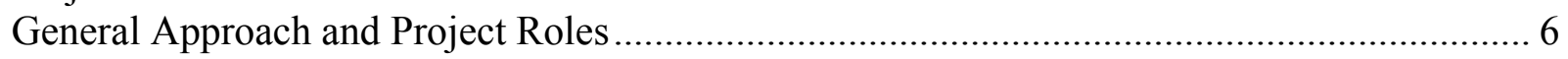

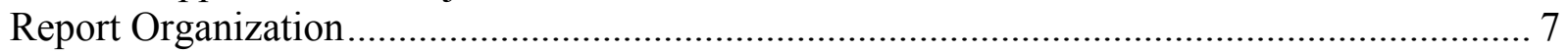

Chapter 2: Past Research and Related Programs ..................................................................... 8

Review of Comprehensive Community-Wide Programs ……………………………......... 10

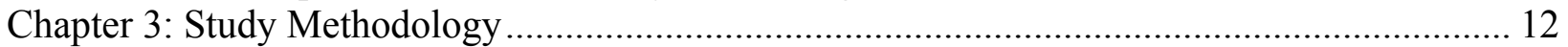

Local Partnership Development...................................................................................... 12

Study Design and Data Sources..................................................................................... 13

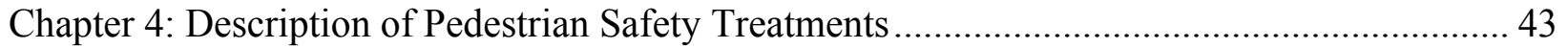

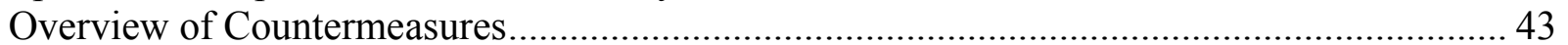

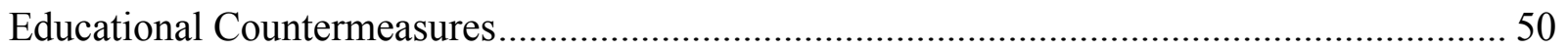

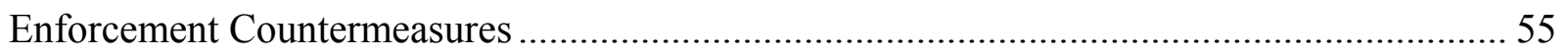

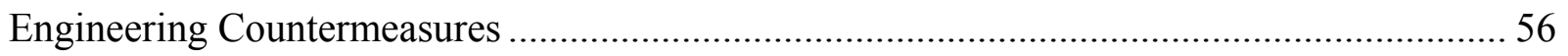

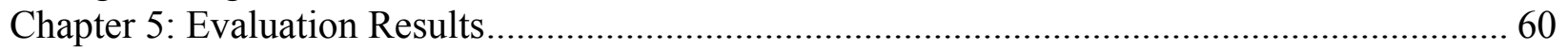

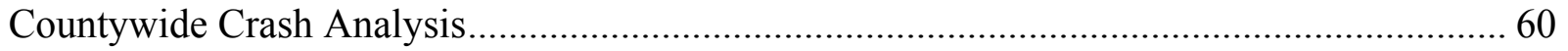

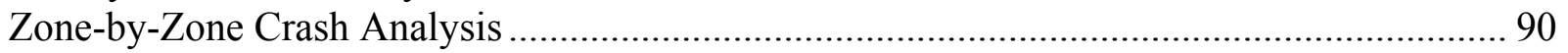

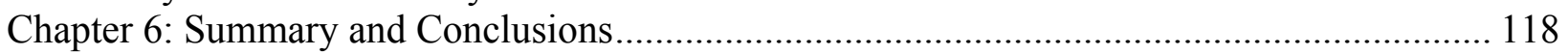

Results of Additional Projects .................................................................................... 124

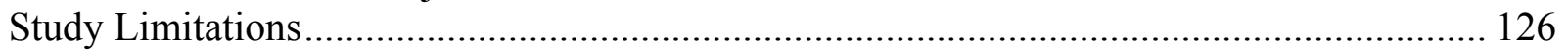

Discussion and Lessons Learned ................................................................................. 128

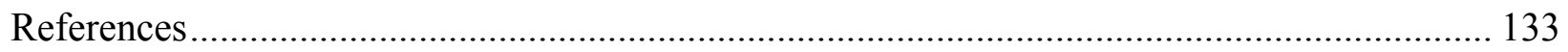

Appendix A: Crash Typing Codes ................................................................................. A-1

Appendix B: Pedestrian Crash Counts and Population For Miami-Dade and Controls .............. B-1

Appendix C: Pedestrian Crash Counts by Age Group, Zone, and Month .................................. C-1

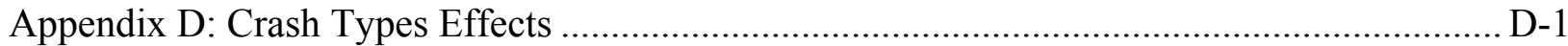

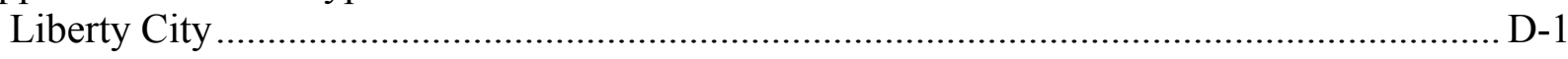

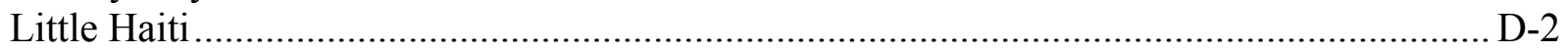

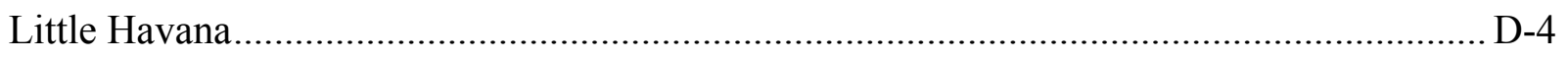

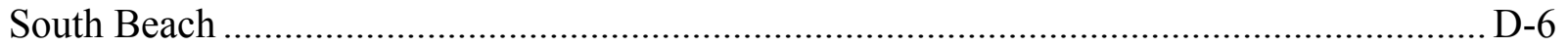




\section{LISTS OF FIGURES}

Figure 1. Miami-Dade High-Pedestrian-Crash Zones. ............................................................ xi Figure 2. Monthly Pedestrian Crashes per 100,000 Population in Miami-Dade County, Broward

County, Six Metropolitan Counties (Combined), and Statewide From 1996-2004 ............ XV

Figure 3. Map of Miami-Dade County, FL ..................................................................... 4

Figure 4. Miami-Dade County Municipalities..................................................................... 5

Figure 5. Map of All Miami-Dade County Pedestrian Crashes, 1996 to 2000 .......................... 18

Figure 6. Pedestrian Crashes by Lighting Conditions. ........................................................... 19

Figure 7. Map of Pedestrian Crashes in Relation to Schools................................................... 20

Figure 8. Map of Pedestrian Crashes in Relation to Nursing Homes. ....................................... 21

Figure 9. Miami-Dade Pedestrian Crash Concentration Areas........................................... 23

Figure 10. Miami-Dade High-Pedestrian-Crash Zones. ..................................................... 25

Figure 11. Pedestrian Crashes by Age of Pedestrian. ........................................................ 26

Figure 12. Pedestrian Crashes by Time of Day. ................................................................. 26

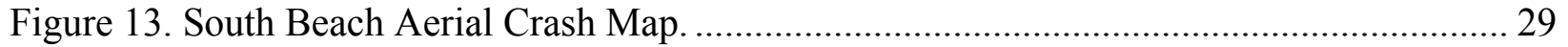

Figure 14. South Beach Crashes by Pedestrian Age.............................................................. 30

Figure 15. South Beach Crashes by Pedestrian Injury Severity. .......................................... 31

Figure 16. Left-Turning Vehicles Failing to Yield to Pedestrians.......................................... 32

Figure 17. Pedestrians Failing to Obey Traffic Signals........................................................ 32

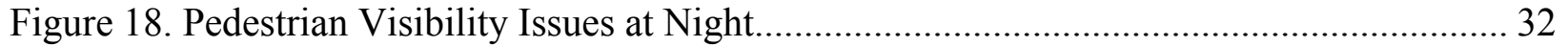

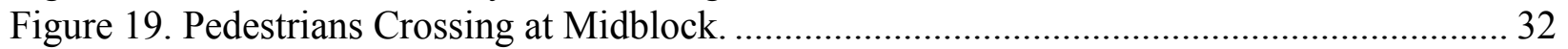

Figure 20. Motorist Failing to Yield to a Pedestrian. ........................................................... 32

Figure 21. Plantings on Bulbouts Blocking Sight Distance................................................... 32

Figure 22. Liberty City/Little Haiti Aerial Crash Map........................................................... 34

Figure 23. Liberty City/Little Haiti Crashes by Pedestrian Age.............................................. 35

Figure 24. Liberty City/Little Haiti Crashes by Pedestrian Injury Severity. ............................. 36

Figure 25. Sight Distance Issues at Intersections................................................................ 37

Figure 26. Middle School Children Walking to School. ........................................................ 37

Figure 27. Pedestrian Crossings at Midblock Locations. ...................................................... 37

Figure 28. Drivers Fail to Yield to Pedestrians in Crosswalks............................................... 37

Figure 29. Pedestrian Signal Violations........................................................................... 37

Figure 30. Children Wait for School Bus in Liberty City..................................................... 37

Figure 31. Little Havana Aerial Crash Map............................................................................ 39

Figure 32. Little Havana Crashes by Pedestrian Age. ............................................................ 40

Figure 33. Little Havana Crashes by Pedestrian Injury Severity........................................... 41

Figure 34. Older Pedestrians Crossing Wide Streets. ....................................................... 42

Figure 35. Pedestrians Violating Walk Signals. .................................................................... 42

Figure 36. Pedestrians Crossing Street to Catch a Bus in Conflict With Cars. ........................... 42

Figure 37. Pedestrian Conflicts With Turning Vehicles........................................................ 42

Figure 38. Pedestrian Conflicts With Bus and Traffic.......................................................... 42

Figure 39. Missing Sidewalk Links and Lack of Facilities for Pedestrians............................. 42

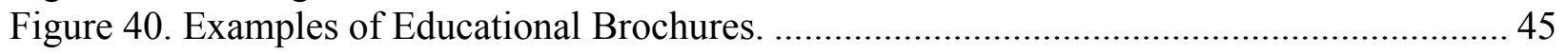

Figure 41. Example Video "La Cita." ............................................................................... 46

Figure 42. Example Walk Safe Miami Posters.................................................................... 47

Figure 43. Countermeasure Implementation Timeline................................................... 49 
Figure 44. Heroes of Haitian Independence Trading Cards. ................................................ 53

Figure 45. Pedestrian Safety Workshop for Older Pedestrians................................................ 54

Figure 46. "Decoy" Pedestrian Crossing the Street in Enforcement Operation. .......................... 55

Figure 47. Enforcement Operations in Miami-Dade. ............................................................ 56

Figure 48. Signs for Drivers to Yield to Pedestrians. ......................................................... 57

Figure 49. Statewide Percentage Distribution of Pedestrian Crashes in Florida by Hour of Day.61

Figure 50. Monthly Numbers of Pedestrian Crashes in Miami-Dade County, Broward County,

Six Metropolitan Counties (Combined), and Statewide From 1996-2004.

Figure 51. Monthly Pedestrian Crashes per 100,000 Population in Miami-Dade County, Broward

County, Six Metropolitan Counties (Combined), and Statewide From 1996-2004. 63

Figure 52. Monthly 1- to 13-Year-Olds' Pedestrian Crashes per 100,000 Population in Miami-

Dade County, Broward County, Six Metropolitan Counties (Combined), and Statewide

From 1996-2004 68

Figure 53. Monthly 5- to 12-Year-Olds' Pedestrian Crashes per 100,000 Population in Miami-

Dade County, Broward County, Six Metropolitan Counties (Combined), and Statewide

From 1996-2004....

Figure 54. Monthly 14- to 64-Year-Olds' Pedestrian Crashes per 100,000 Population in Miami-

Dade County, Broward County, Six Metropolitan Counties (Combined), and Statewide

From 1996-2004. 72

Figure 55. Monthly 65-and-Older Pedestrian Crashes per 100,000 Population in Miami-Dade

County, Broward County, Six Metropolitan Counties (Combined), and Statewide From 1996-2004.

Figure 56. Monthly Male Pedestrian Crashes per 100,000 Population in Miami-Dade County,

Broward County, Six Metropolitan Counties (Combined), and Statewide from 1996-2004.

Figure 57. Monthly Female Pedestrian Crashes per 100,000 Population in Miami-Dade County,

Broward County, Six Metropolitan Counties (Combined), and Statewide from 1996-2004.

Figure 58. Monthly 6 a.m. to 9:59 a.m. Pedestrian Crashes per 100,000 Population in Miami-

Dade County, Broward County, Six Metropolitan Counties (Combined), and Statewide

From 1996-2004.....

Figure 59. Monthly 10 a.m. to 1:59 p.m. Pedestrian Crashes per 100,000 Population in Miami-

Dade County, Broward County, Six Metropolitan Counties (Combined), and Statewide

from 1996-2004.

Figure 60. Monthly 2 p.m. to 5:59 p.m. Pedestrian Crashes per 100,000 Population in Miami-

Dade County, Broward County, Six Metropolitan Counties (Combined), and Statewide

From 1996-2004.

Figure 61. Monthly 6 p.m. to 9:59 p.m. Pedestrian Crashes per 100,000 Population in Miami-

Dade County, Broward County, Six Metropolitan Counties (Combined), and Statewide

from 1996-2004.

Figure 62. Monthly 10 p.m. to 5:59 a.m. Pedestrian Crashes per 100,000 Population in Miami-

Dade County, Broward County, Six Metropolitan Counties (Combined), and Statewide

from 1996-2004.

Figure 63. Crash Zone Locations in Miami-Dade County, Florida.

Figure 64. Miami-Dade County Pedestrian Crash Density Map (Kernel density estimation; bandwidth $=0.5$ mile) 
Figure 65. Miami-Dade County Pedestrian Crash Density Map (Kernel density estimation;

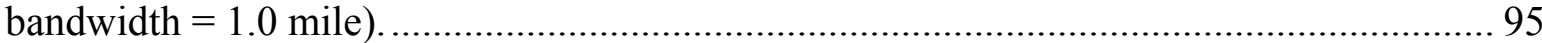

Figure 66. Crash Density Map of Four Crash Zones (Kernel density estimation; bandwidth $=0.2$ mile). 96

Figure 67. Liberty City Crash Density Map (Kernel density estimation; bandwidth $=0.2$ mile). 97 Figure 68. Little Haiti Crash Density Map (Kernel density estimation; bandwidth $=0.2$ mile). . 98 Figure 69. Little Havana Crash Density Map (Kernel density estimation; bandwidth $=0.2$ mile). 99

Figure 70. South Beach Crash Density Map (Kernel density estimation; bandwidth $=0.2$ mile) .

Figure 72. Liberty City Before-After Comparison of the Cell-Based Crash Density Values. ... 103 Figure 73. Little Haiti Before-After Comparison of the Cell-Based Crash Density Values. ..... 104 Figure 74. Little Havana Before-After Comparison of the Cell-Based Crash Density Values. . 105 Figure 75. South Beach Before-After Comparison of the Cell-Based Crash Density Values.... 106 Figure 76. Monthly Pedestrian Crash Frequency by Crash Zones (1996-2004). ....................... 107 Figure 77. Pedestrian Crash Frequency by Age Groups in Liberty City (1996-2004)................ 108 Figure 78. Pedestrian Crash Frequency by Age Groups in Little Haiti (1996-2004)................. 109

Figure 79. Pedestrian Crash Frequency by Age Groups in Little Havana (1996-2004)............. 110 Figure 80. Pedestrian Crash Frequency by Age Groups in South Beach (1996-2004). ............. 111

Figure 81. Crash Frequency Involving School Kids (Age 5 to 12) by Zones (1996-2004). ...... 113 Figure 82. Crash Frequency Involving Adult Pedestrians (Age 14 to 64) by Zones (1996-2004).

Figure 83. Crash Frequency Involving Older Pedestrians (Age 65 and Older) by Zones (19962004). 


\section{LISTS OF TABLES}

Table 1. List of Countermeasures Implemented....................................................................

Table 2. Mean Difference in Monthly Crashes From Before Period to After Period................. xviii

Table 3. Common Crash Types and Descriptions. ..................................................................... 1

Table 4. Summary of Pedestrian-Motor Vehicle Crashes by Year for the Florida DHSMV

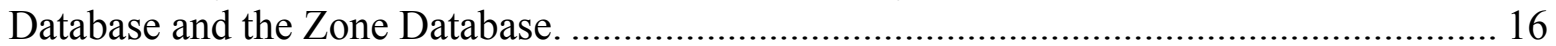

Table 5. Annual Crash Frequency by Year and Crash Zones........................................................ 24

Table 6. Countermeasure Locations in Miami-Dade County.......................................................5 58

Table 7. Unadjusted Post-Intervention Percentage Difference in Overall Average Monthly

Pedestrian Crash Rates (per 100,000 Population) in Each Florida Jurisdiction by Intervention Date, 1996-2004.

Table 8. Summary of Sudden-Permanent ARIMA Models of Effect in Miami-Dade County for Overall Pedestrian Crash Rates per 100,000 Population Using Three Different Control Series.

Table 9. Re-Estimated Intervention Effect Sizes for the January 2003 Intervention Point on Overall Miami-Dade Pedestrian Crashes per 100,000 Population Using Three Different Control Series.

Table 10. Unadjusted Post-Intervention Percentage Difference in 1- to 13-Year-Olds' Average Monthly Pedestrian Crash Rates in Each Florida Jurisdiction by Intervention Date, 19962004

Table 11. Summary of Sudden-Permanent ARIMA Models of Effect in Miami-Dade County for 1- to 13-Year-Olds' Pedestrian Crash Rates per 100,000 Population Using Three Different Control Series.

Table 12. Unadjusted Post-Intervention Percentage Difference in 5- to 12-Year-Olds' Average Monthly Pedestrian Crash Rates in Each Florida Jurisdiction by Intervention Date, 19962004

Table 13. Summary of Sudden-Permanent ARIMA Models for 5- to 12-Year-Olds' Pedestrian Crash Rates per 100,000 Population Using Three Different Control Series.

Table 14. Unadjusted Post-Intervention Percentage Difference in 14- to 64-Year-Olds' Average Monthly Pedestrian Crash Rates in Each Florida Jurisdiction by Intervention Date, 19962004

Table 15. Summary of Sudden-Permanent ARIMA Models of Effect in Miami-Dade County for 14- to 64-Year-Olds' Pedestrian Crash Rates per 100,000 Population Using Three Different Control Series 74

Table 16. Unadjusted Post-Intervention Percentage Difference in 65+ Age Group Average Monthly Pedestrian Crash Rates in Each Florida Jurisdiction by Intervention Date, 19962004

Table 17. Summary of Sudden-Permanent ARIMA Models of Effect in Miami-Dade County for 65-and-Older Pedestrian Crash Rates per 100,000 Population Using Three Different Control Series. 76

Table 18. Unadjusted Post-Intervention Percentage Difference in Male Average Monthly Pedestrian Crash Rates in Each Florida Jurisdiction by Intervention Date, 1996-2004....... 77 
Table 19. Summary of Sudden-Permanent ARIMA Models of Effect in Miami-Dade County for Male Pedestrian Crash Rates per 100,000 Population Using Three Different Control Series.

Table 20. Unadjusted Post-Intervention Percentage Difference in Female Average Monthly

Pedestrian Crash Rates in Each Florida Jurisdiction by Intervention Date, 1996-2004...... 79

Table 21. Summary of Sudden-Permanent ARIMA Models of Effect in Miami-Dade County for

Female Pedestrian Crash Rates per 100,000 Population Using Three Different Control Series.

Table 22. Unadjusted Post-Intervention Percentage Difference in 6 a.m. to 9:59 a.m. Average Monthly Pedestrian Crash Rates in Each Florida Jurisdiction by Intervention Date, 19962004

Table 23. Summary of Sudden-Permanent ARIMA Models of Effect in Miami-Dade County for 6 a.m. to 9:59 a.m. Pedestrian Crash Rates per 100,000 Population Using Three Different Control Series.

Table 24. Unadjusted Post-Intervention Percentage Difference in 10 a.m. to 1:59 p.m. Average

Monthly Pedestrian Crash Rates in Each Florida Jurisdiction by Intervention Date, 19962004

Table 25. Summary of Sudden-Permanent ARIMA Models of Effect in Miami-Dade County for 10 a.m. to 1:59 p.m. Pedestrian Crash Rates per 100,000 Population Using Three Different Control Series.

Table 26. Unadjusted Post-Intervention Percentage Difference in 2 p.m. to 5:59 p.m. Average Monthly Pedestrian Crash Rates in Each Florida Jurisdiction by Intervention Date, 19962004

Table 27. Summary of Sudden-Permanent ARIMA Models of Effect in Miami-Dade County for 2 p.m. to 5:59 p.m. Pedestrian Crash Rates per 100,000 Population Using Three Different Control Series. 86

Table 28. Unadjusted Post-Intervention Percentage Difference in 6 p.m. to 9:59 p.m. Average Monthly Pedestrian Crash Rates in Each Florida Jurisdiction by Intervention Date, 19962004

Table 29. Summary of Sudden-Permanent ARIMA Models of Effect in Miami-Dade County for 6 p.m. to 9:59 p.m. Pedestrian Crash Rates per 100,000 Population Using Three Different Control Series.

Table 30. Unadjusted Post-Intervention Percentage Difference in 10 p.m. to 5:59 a.m. Average Monthly Pedestrian Crash Rates in Each Florida Jurisdiction by Intervention Date, 19962004

Table 31. Summary of Sudden-Permanent ARIMA Models of Effect in Miami-Dade County for 10 p.m. to 5:59 a.m. Pedestrian Crash Rates per 100,000 Population Using Three Different

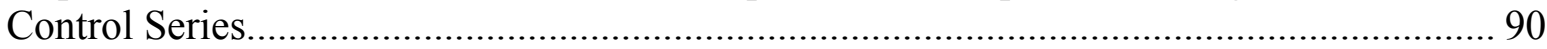

Table 32. Pedestrian Crash Frequency by Crash Zones (1996-2004). .................................... 92

Table 33. Mean Difference in Monthly Crashes from Before Period to After Period................ 112

Table 34. Summary of Results From the Countywide Analysis............................................ 122

Table 35. Liberty City Overall Crash Group Frequencies and Percentages by Year................... 2

Table 36. Little Haiti Overall Crash Group Frequencies and Percentages by Year. .................... 4

Table 37. Little Havana Overall Crash Group Frequencies and Percentages by Year................. 5

Table 38. South Beach Overall Crash Group Frequencies and Percentages by Year.................... 7 


\section{TECHNICAL SUMMARY}

\section{Background}

The number of pedestrians killed in United States traffic crashes has declined over $40 \%$ since peaking in the late 1970s and early 1980s. Still, in 2005 there were 4,881 recorded pedestrian fatalities, representing $11 \%$ of all U.S. traffic deaths (NHTSA, 2006, Table 53). In urban areas where pedestrian activity and traffic volumes are greater compared to rural areas, pedestrians often comprise $25 \%$ of traffic deaths or more.

During the 1970s, a research project series sought to identify causal factors of pedestrian crashes and appropriate countermeasures. The research by Snyder and Knoblauch (1971) focused on urban pedestrian crashes, but subsequent studies extended the methodology to rural and freeway crashes (see Stutts et al., 1992, for a review). From this research evolved the basic pedestrian crash "typology" to describe behaviorally similar pre-crash actions that lead to characteristic pedestrian-motor vehicle collisions. The aim in typing crashes is to gain a better understanding of underlying factors and causes so that appropriate countermeasures can be developed.

During the late 1990s, the National Highway Traffic Safety Administration also developed the concept of pedestrian safety zones, to focus improvements where the problem is greatest. By concentrating efforts where the majority of the problem or the target audience exists, funds are used more efficiently and activities that would be prohibitively expensive if applied to an entire community can be applied for greatest benefit on a smaller scale. For example, in Phoenix six circular zones and one linear zone were identified that accounted for $54.9 \%$ of the city's olderadult crash population in about $4.6 \%$ of the land area (Blomberg \& Cleven, 1998).

\section{Project Goals and Objectives}

The overall goal was to reduce pedestrian deaths and injuries in a large urban environment by implementing a long-term pedestrian safety program within the jurisdiction. The specific project objectives were to work with stakeholders in the community to:

1. Obtain and analyze pedestrian crash data to identify zones of high incidence of pedestrian crashes and the special characteristics of those crashes;

2. Identify and implement a comprehensive program of educational, engineering, and enforcement strategies (see Chapter 4 for details) to address the problems identified;

3. Evaluate the safety benefits of the program; and

4. Document the process for other cities or urban areas that may want to replicate the process. 


\section{Project Site}

The project was conducted in Miami-Dade County, Florida. This location was selected because of the significant pedestrian injury and fatality problems the area was experiencing. In 2001, just before the project began, Florida was the fourth-largest State in terms of population (16.4 million), but ranked first in the number of pedestrian fatalities (489). In Florida, Miami-Dade County (in 2001) led the State in pedestrian deaths and injuries.

In addition to its large urban population (2.4 million people) and significant pedestrian safety problem, there were a number of other reasons that led to the site choice, including excellent sources of available data, multidisciplinary interest among local agencies, and strong leadership and support from the State and county level.

\section{Study Methodology}

\section{Local Partnership Development}

Several partnerships were developed between local, regional, and State agencies to promote a sustainable program and capitalize on existing activities. Key partners included departments of transportation, injury prevention coalitions, the Miami-Dade County Metropolitan Planning Organization (MPO), and others.

\section{Study Design and Data Sources}

This study reports on the results of a comprehensive pedestrian safety program implemented in Miami-Dade County. The design for the Miami-Dade pedestrian demonstration included the use of pedestrian crashes as the sole outcome measure of effectiveness. While intermediate measures of pedestrian and driver behavior have been used in other evaluation studies of pedestrian countermeasures, they were not feasible in the current effort for two reasons. First, the interventions in the project were to be comprehensive and therefore would cover a multitude of potentially relevant behaviors, most of which would be difficult or costly to measure in a valid and reliable manner. Second, the available pedestrian crash data for Miami-Dade, both baseline and post-interventions, were sufficiently large to support a sensitive assessment of program success based on the ultimate crash reduction outcome measure.

With crashes as the project's evaluation measure, effort turned to building a database of crashes to support analyses of effectiveness. This involved selecting a source for crash data, retrieving and coding the data, and defining appropriate subsets for analysis for the years 1996-2004.

\section{$\underline{\text { Data Collection and Processing }}$}

The Florida Department of Highway Safety and Motor Vehicles (DHSMV) database consisting of all the State's reported pedestrian-related crashes was the original data source used for the countywide crash evaluation. Additional effort was required to refine the data, assign crash types using Pedestrian and Bicycle Crash Analysis Tool (PBCAT) software, and perform address 
matching to locate the crash event within the electronic street map of a geographic information system (GIS).

\section{Data Sample}

Over the nine years of the project period examined, there were 17,308 total pedestrian crashes in the DHSMV Miami-Dade County, which included 724 fatal crashes (4.2\%). After screening and geocoding all crash reports, a total of 15,472 pedestrian-motor vehicle crashes remained, which were used in the zone analysis. Of these, there were 670 fatal injuries reported and 3,002 crashes involving serious injury. Crashes fluctuated during the years of the study period, with an apparent downward trend in total crashes from 1996 to 1999, before the program countermeasures were implemented. Most of the countermeasures were implemented after January of 2002, so the "before period" used in the analysis is 1996 through 2001, and the "after period" is 2002 through 2004. The year 2002 was selected as the first year of the after period since some countermeasures were implemented near the beginning of 2002.

\section{Generating Pedestrian Crash Maps}

The team produced maps of pedestrian crashes for the before-analysis period of 1996-2001. A crash location (pin) map was analyzed to reveal different crash-related factors, such as age of pedestrian, injury severity, light conditions, and crash type, based on data from the police report. The pedestrian crash data was combined with other Miami-Dade County GIS data to show the relationship of crashes to other spatial data, such as locations of schools, nursing homes, transit stops, and aerial images. These and other pedestrian crash maps were also later used at the zone level (see next section) in the process of countermeasure development.

\section{$\underline{\text { Identification of High-Crash Zones and Problems }}$}

Crash density per acre was calculated using GIS to identify high-crash areas, corridors, and intersections for prioritizing countermeasure resources. Based on this analysis, four zones were identified for further investigation and targeted pedestrian safety measures. These zones included South Beach, Liberty City, Little Havana, and Little Haiti, shown in Figure 10. The area size of the identified four zones is 9,891 acres, less than $1 \%$ of the total area size of Miami-Dade County. However, from 1996 to 2004, the number of pedestrian crashes in the four zones comprised about $20 \%$ (3,078 of 15,474 crashes) of the total number of pedestrian crashes in Miami-Dade County. 


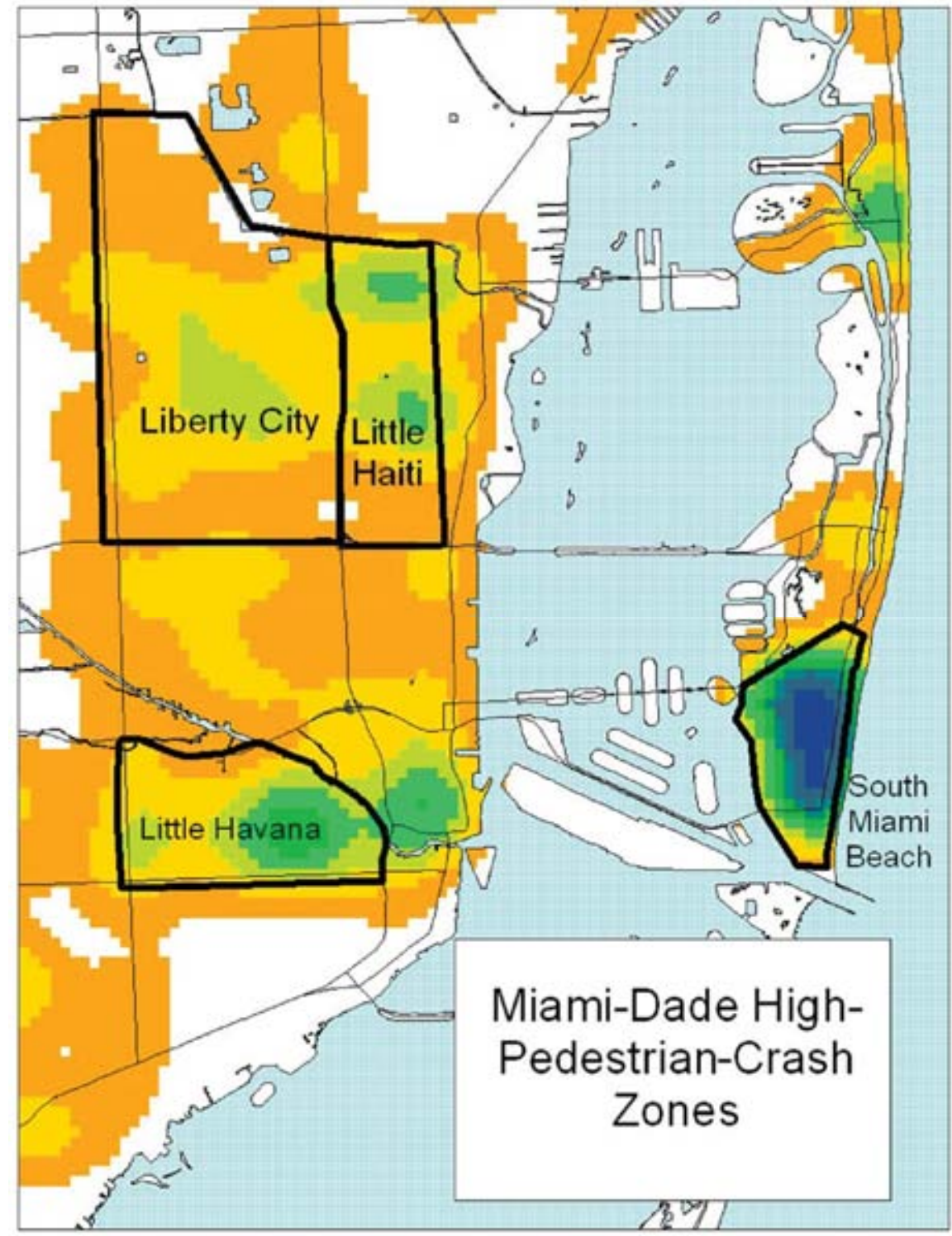

Figure 1. Miami-Dade High-Pedestrian-Crash Zones.

For each zone, detailed crash maps were generated to show the pedestrian crash patterns that have occurred along various corridors and at certain intersections. Crash data were further analyzed by mapping crashes by pedestrian age, time of day, and other factors. This analysis revealed several general trends that further distinguished each study zone's pedestrian problems.

In each of the four identified high-crash zones, a detailed review was conducted of the crash maps plus individual police crash reports. Project team members also conducted on-site investigations of the high-crash zones and visited many of the high-crash corridors and locations within each of these zones. Site reviews included observing motorist and pedestrian behaviors, identifying obvious or potentially problematic roadway features that could contribute to pedestrian crashes; and listing potential engineering, education, and enforcement treatments. 


\section{Description of Study Zones}

The four zones had several common pedestrian safety and operational issues, some of which were addressed through engineering treatments after the end of this study period. The zones also had many behavior-related concerns in common, such as:

- Motorists failing to yield to pedestrians in crosswalks and at unsignalized intersections;

- Motorists running red signals, particularly those making turns on red;

- Pedestrians walking or running into the street at midblock in front of on-coming traffic;

- Unaccompanied young school children walking to school and crossing wide streets; and

- Pedestrians crossing against the traffic signal or at midblock between parked cars.

Below is a brief description of some of the specific issues within each of the zones:

- South Beach - Many crashes involved young adults and older pedestrians, and a highnight-time-crash problem.

- Liberty City and Little Haiti-Many crashes involved young children who were struck by motor vehicles. However, some of the crashes involved adults and older adults, particularly those trying to cross wide (4- and 5-lane) streets.

- Little Havana-Little Havana's population is largely Hispanic, with a substantial percentage of people of Cuban origin. A high percentage of the pedestrian crashes involved older pedestrians of Hispanic descent.

These safety concerns helped local, county, and State officials determine which countermeasures were needed in each zone.

\section{Description of Pedestrian Safety Treatments}

Drawing on the pedestrian safety issues identified, a total of 16 pedestrian safety treatments were targeted to areas within Miami-Dade County, and particularly within the four selected zones (see Table 1 for details). Countermeasure implementation began at different times and many have continued beyond the end point of the project. 
Table 1. List of Countermeasures Implemented.

\begin{tabular}{|c|c|c|c|}
\hline \# & Countermeasure & $\begin{array}{l}\text { Start } \\
\text { Year }\end{array}$ & Location* \\
\hline \multicolumn{4}{|c|}{ Educational Countermeasures } \\
\hline 1 & $\begin{array}{l}\text { WalkSafe Program and Ryder Trauma Center Classroom Education-PProgram } \\
\text { aimed at reducing the incidence of children struck by vehicles by educating } \\
\text { elementary-school-age children and their teachers, parents, and communities about } \\
\text { traffic safety. The program used an educational training intervention, appropriate } \\
\text { engineering countermeasures, and an enforcement component to help achieve its goal. } \\
\text { An evaluation of the program can be found in Hotz et al. (2004). }\end{array}$ & 2003 & LC, $\mathrm{LH}$ \\
\hline 2 & $\begin{array}{l}\text { Pedestrian Safety Message Mounted in Bus and Metrorail Train Posters- } \\
\text { Included six sets of different pedestrian education posters aimed at increasing } \\
\text { pedestrian safety practices, including safe practices when walking at night. The } \\
\text { posters' safety messages were in English, Spanish, and Creole. The target audiences } \\
\text { were primarily adults. }\end{array}$ & 2003 & $\begin{array}{l}\text { County- } \\
\text { wide }\end{array}$ \\
\hline 3 & $\begin{array}{l}\text { Walk to School Day Sponsored by SAFE KIDS Walk This Way-Thousands of } \\
\text { students from } 8 \text { schools participated in Walk to School Day. The National SAFE KIDS } \\
\text { Campaign provided banners, signs, pedestrian safety pamphlets, and walkability } \\
\text { surveys. Over } 100,000 \text { copies of the "Walking Through the Years" brochure were } \\
\text { distributed at events from } 2001 \text { to } 2005 \text {, as well as } 10,000 \text { retro-reflective zipper pulls } \\
\text { and wrist bands. }\end{array}$ & 1999 & $\begin{array}{l}\text { County- } \\
\text { wide }\end{array}$ \\
\hline 4 & $\begin{array}{l}\text { Pedestrian Education by the Community Affairs Bureau of the Miami-Dade } \\
\text { Police Department--The Pedestrian Safety Section of the Miami-Dade Police } \\
\text { Department's Community Affairs Bureau made numerous traffic safety presentations } \\
\text { in schools, distributed several safety booklets and material, and helped establish the } \\
\text { WalkSafe Miami program. The target audiences were primarily children. }\end{array}$ & 1999 & $\begin{array}{l}\text { County- } \\
\text { wide }\end{array}$ \\
\hline 5 & $\begin{array}{l}\text { Haitian Creole Elementary School and Older Pedestrian Safety Education } \\
\text { Programs-The elementary school program consisted of four 45-minute workshops } \\
\text { conducted at three elementary schools, reaching } 389 \text { children. Both programs were } \\
\text { supported by radio advertisements, Haitian Web sites, a brochure in Haitian Creole, } \\
\text { and Haitian Creole trading cards. }\end{array}$ & 2001 & $\mathrm{LC}$ \\
\hline 6 & $\begin{array}{l}\text { Brochure: Safety Tips for Pedestrians in Haitian Creole-Pamphlet that provides } \\
\text { pedestrian safety advice to adults. These were handed out at senior centers and social } \\
\text { service providers. }\end{array}$ & 2002 & $\mathrm{LC}$ \\
\hline 7 & $\begin{array}{l}\text { Heroes of Haitian Independence Trading Cards-Four cards that each depict a hero } \\
\text { of Haitian independence on one side and provide pedestrian safety tips on the other. } \\
\text { These were distributed at senior centers, schools, and health fair events. }\end{array}$ & 2002 & $\mathrm{LC}$ \\
\hline 8 & $\begin{array}{l}\text { Public Service Announcements (PSAs)—PSAs about pedestrian safety were } \\
\text { distributed and broadcasted on city and county access channels in Spanish and English } \\
\text { and on selected Spanish-speaking radio stations. }\end{array}$ & 2003 & $\begin{array}{l}\text { County- } \\
\text { wide }\end{array}$ \\
\hline 9 & $\begin{array}{l}\text { Brochure: Pedestrian, Walk Safely-Brochure providing families with the } \\
\text { pedestrian safety advice in both English and Spanish. Brochures were delivered to } \\
\text { organizations such as the Miami-Dade School Board, hospitals, public libraries, police } \\
\text { departments, and elected officials' offices. }\end{array}$ & 2002 & LC, LH \\
\hline 10 & $\begin{array}{l}\text { Walking Through the Years: Pedestrian Safety for Your Child-Brochure (in } \\
\text { English and Spanish) providing safety guidelines to parents and caregivers to help } \\
\text { protect children from pedestrian crashes. Brochures were delivered to organizations } \\
\text { such as the Miami-Dade School Board, hospitals and medical departments, public } \\
\text { libraries, police departments, and elected officials' offices. }\end{array}$ & 2002 & LC, LH \\
\hline 11 & $\begin{array}{l}\text { Pedestrian Safety Workshops for Older Populations-The Miami-Dade MPO } \\
\text { pedestrian-bicycle coordinator began providing workshops on pedestrian safety to older } \\
\text { pedestrians and groups working with older populations in 2002. Presentations were } \\
\text { made at more than } 20 \text { assemblies and senior health fair events. }\end{array}$ & 2002 & $\begin{array}{l}\text { SB, LC, } \\
\text { LH }\end{array}$ \\
\hline
\end{tabular}




\begin{tabular}{|c|c|c|c|}
\hline \# & Countermeasure & $\begin{array}{l}\text { Start } \\
\text { Year }\end{array}$ & Location* \\
\hline \multicolumn{4}{|c|}{$\begin{array}{c}\text { Educational Countermeasures } \\
\end{array}$} \\
\hline 12 & $\begin{array}{l}\text { Walking Through the Years: Pedestrian Safety for the Older Adult-Booklet } \\
\text { prepared for older }(65+) \text { adults and implementers of programs for older adults. } \\
\text { Brochures were delivered to organizations such as the Miami-Dade School Board, } \\
\text { hospitals and medical departments, retirement homes, public libraries, police } \\
\text { departments, and older affairs and elected officials' offices. }\end{array}$ & 2002 & SB, LH \\
\hline 13 & $\begin{array}{l}\text { Caminando a Traves de los Anos: Seguridad para Peatones de Tercera Edad } \\
(65+) \text {-Booklet in Spanish prepared for implementers of pedestrian programs for the } \\
\text { older }(65+) \text { adult. Brochures were delivered to organizations such as the Miami-Dade } \\
\text { School Board, hospitals, retirement homes, public libraries, police departments, and } \\
\text { older affairs and elected officials' offices. }\end{array}$ & 2002 & SB, LH \\
\hline 14 & $\begin{array}{l}\text { Nighttime Conspicuity Enhancements-More than } 400 \text { posters on nighttime } \\
\text { conspicuity related to pedestrian safety were distributed to organizations to display in } \\
\text { public buildings. }\end{array}$ & 2002 & $\begin{array}{l}\text { SB, LC, } \\
\text { LH }\end{array}$ \\
\hline \multicolumn{4}{|c|}{ Enforcement Countermeasures } \\
\hline 15 & $\begin{array}{l}\text { Enforcement of Driver Yielding Behavior Study, Two Police Pedestrian Safety } \\
\text { Training Programs, and Enforcement-Van Houten and Malenfant (2003) } \\
\text { conducted a study of driver yielding behavior and enforcement at four crosswalks in } \\
\text { two high-crash corridors in Miami Beach. For enforcement results, review Van Houten } \\
\text { and Malenfant (2003). Additionally, police officers in Miami Beach and Miami } \\
\text { Springs received training on pedestrian safety and enforcement activities. }\end{array}$ & 2002 & SB \\
\hline \multicolumn{4}{|c|}{ Engineering Countermeasures } \\
\hline 16 & $\begin{array}{l}\text { Florida Department of Transportation Engineering Projects Related to } \\
\text { Pedestrians-During the implementation period of January } 2002 \text { through December } \\
31,2004 \text {, numerous engineering and roadway treatments were implemented by FDOT. } \\
\text { These included measures such as adding raised medians on selected multilane roads, } \\
\text { installing missing sidewalk links, installing pedestrian warning signs at specific } \\
\text { locations, revising traffic signal timing, implementing safer facilities in selected school } \\
\text { zones, and others. Over } \$ 6.5 \text { million in pedestrian safety projects were programmed or } \\
\text { implemented on } 12 \text { corridors. }\end{array}$ & 2002 & $\begin{array}{l}\text { County- } \\
\text { wide }\end{array}$ \\
\hline
\end{tabular}

\section{Analysis and Results}

\section{Countywide Crash Evaluation Results}

For the countywide pedestrian crash evaluation, several control groups were identified to remove the effects of preexisting downward trends and other changes that could be mistaken for program effects. These control groups included Broward County (the county just north of Miami-Dade County that includes Ft. Lauderdale), the six metropolitan counties in Florida (Duval, Hillsborough, Pinellas, Palm Beach, Orange, and Broward Counties, combined), and all Florida pedestrian crashes (excluding Miami-Dade County).

It was not considered feasible to determine the effect of each of the individual countermeasures on pedestrian crashes, since several of the treatments had similar or overlapping implementations and target populations. Thus, the evaluation focused on the overall pedestrian safety program. Multivariate intervention auto-regressive integrated moving average (ARIMA) time series analysis was used to determine the overall impact of the program. 
To account for changes in the underlying population, the monthly counts were turned into rates per 100,000 population. The monthly total pedestrian crash rates are illustrated in Figure 51. The 12 -month moving average in each series is also shown to help with interpreting or identifying the trends.

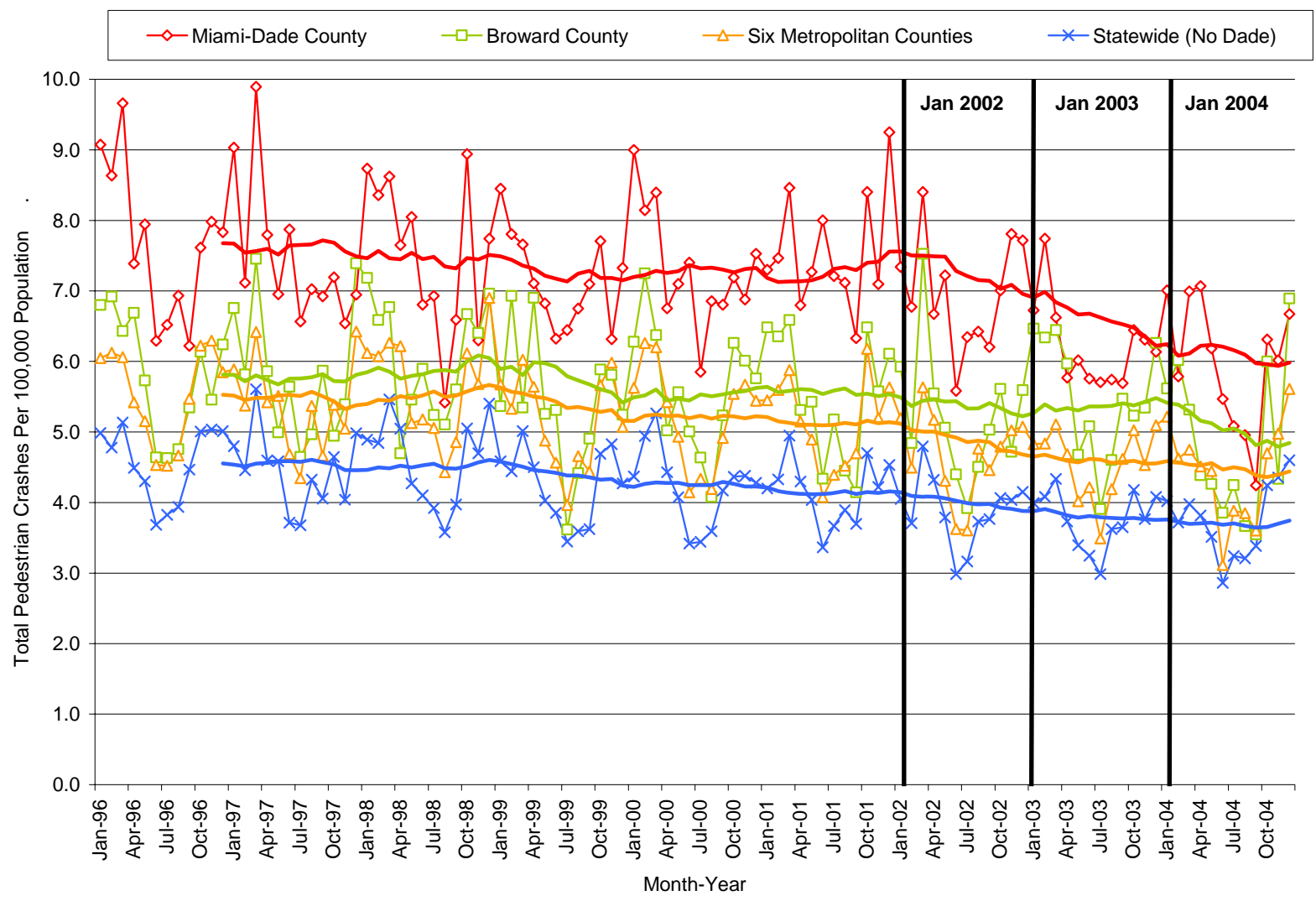

Figure 2. Monthly Pedestrian Crashes per 100,000 Population in Miami-Dade County, Broward County, Six Metropolitan Counties (Combined), and Statewide From 1996-2004.

Miami-Dade had higher pedestrian crash rates than any of the other series, including the rest of Florida. The decrease in pedestrian crashes in Miami-Dade County is much more apparent in the per-capita figure, and it does appear to coincide with the time period during which the pedestrian safety program was underway. However, also apparent in the other control series are downward trends in pedestrian crashes that began some time before the interventions in Miami-Dade County. This downward trend is also apparent in the Miami-Dade County series, but is much more gradual than the sharp decrease in the pedestrian crash rate that began in early 2002. It is unknown why pedestrian crash rates were slowly decreasing in Florida during the time period shown, but some evidence shows that the drop could partly be a sign of decreased walking activities. Census data show that from 1990 to 2000, the percentage of people walking to work dropped from $2.51 \%$ to $1.71 \%$ in Florida. In Miami-Dade County, the percentage of people walking to work dropped from $2.53 \%$ to $2.15 \%$ (Census, 2000). It was important to remove this trend from the Miami-Dade County series before evaluating the effect of the interventions; thus, control series were included in the analyses. Also note that the pedestrian crash rate in Miami- 
Dade actually appears to have leveled off in early 2001 and then began to increase in the later half of 2001. At this point there is a "bump" in the pedestrian crash rate. A similar bump can be seen in the Broward County series around January 2003. In other words, the pedestrian crash rate in Miami-Dade County was increasing in late 2001 before the countermeasure program was implemented, beginning in early 2002.

To determine the overall impact of the pedestrian safety program, ARIMA time series analysis was used (Box \& Jenkins, 1970; Box \& Tiao, 1975). Based on the timeline in which interventions were implemented in Miami-Dade County, three different intervention points were tested in each model: (a) January 2002, (b) January 2003, and (c) January 2004.

Below is a summary of the key findings from the ARIMA countywide time series evaluations:

1. The first significant effect of the pedestrian safety program on overall pedestrian crashes was the intervention point in January 2003. The total effect of the Miami-Dade pedestrian safety program was estimated to be a $13.3 \%$ reduction in pedestrian crashes based on using Broward County as a control series, and an $8.5 \%$ reduction based on using the six metropolitan counties or the statewide crash rates as control series. These reductions were significant at the .05 level. The benefits of the pedestrian safety program continued beyond 2003 in that the average number of pedestrian crashes in 2004 remained lower than the pre-2003 level. However, there was no independent additive reduction detected that could be associated with the pedestrian safety activities conducted during 2004. The ARIMA analyses showed that there was a large reduction in pedestrian crashes in MiamiDade County during the combined 2003-to-2004 time period after adjusting for other temporal trends (e.g., fuel prices and changes in traffic safety laws) and seasonality using the various comparison series of Florida jurisdictions. The conclusion that this reduction can largely be attributed to the overall pedestrian safety program is supported by the fact that the reductions in Miami-Dade pedestrian crashes were consistently larger than those for other Florida jurisdictions, regardless of how the comparison group was formed.

Thus, pedestrian crashes in Miami-Dade County were reduced by about 180 per year for a total of 360 fewer pedestrian crashes during the two-year 2003 and 2004 "after" period. A possible cause of this reduction is the combined pedestrian safety program efforts that began in 2003. The fact that pedestrian crashes per month leveled off during 2004 may indicate that additional countermeasures (or increased countermeasure intensity) are needed to achieve additional reductions in the monthly rate of pedestrian crashes after 2004 , or that additional data points are necessary to be able to detect any additional independent effect of the activities in 2004.

2. Several of the countermeasures that were part of the overall pedestrian safety program were directed at reducing crashes among children. One of the primary countermeasures was the "WalkSafe" program, which was a countywide pedestrian safety education program implemented in virtually all of the Miami-Dade County elementary schools. Examination of pedestrian crashes for children (considering ages 1 to 13 and 5 to 12 separately, to better account for elementary age children affected by the intervention) showed mixed results on a countywide basis. Although Miami-Dade experienced a large 
decrease in pedestrian crashes among children after January 2003, so did some of the control jurisdictions. Specifically, the analysis results showed a significant reduction in child pedestrian crashes as of January 2003, using Broward County as the control series, which would correspond to an $18.5 \%$ decrease. However, the results of the analysis did not indicate a significant change (.05 level) in the child pedestrian crash rates using the six metropolitan county or statewide control series. This result was clearly affected by the continuing drop in child pedestrian crashes statewide and in the six metro areas, particularly since October 2000.

Such gradual but steady reductions in crashes in these two control groups may have been the result of factors such as less walking exposure (e.g., fewer children walking to school) and/or the result of statewide pedestrian safety initiatives carried out by FDOT in recent years. The Miami-Dade pedestrian safety education program "WalkSafe" was initiated in the latter part of 2003, and thus the full benefit of the educational program may have occurred later than the January 2003 intervention period. More discussion of such an evaluation for the high-crash zones is provided later.

3. The ARIMA analysis of 14- to 64-year-olds' pedestrian crash rates indicated a significant reduction among this age group in Miami-Dade County starting in January 2003, regardless of the control group used. There was a downward trend in crashes involving this age group in each of the control groups, as well as a steeper downward trend in Miami-Dade County. Using the statewide control series to estimate the magnitude of this effect, the 2003 intervention date was associated with a 0.60 monthly reduction in Miami-Dade 14- to 64 -year-olds' pedestrian crashes per 100,000 population, or about an $8.6 \%$ annual reduction in the average level prior to the pedestrian safety program.

4. The average crash rate for older pedestrians (those 65 and older) was lower in MiamiDade County and also in each of the control groups in the after period compared to the before period. None of the ARIMA models, however, indicated a significant change in the 65-and-older pedestrians' rates in Miami-Dade County at any of the intervention points after controlling for variability using the control series. More discussion on this issue is provided later, particularly with respect to Little Havana, where several countermeasures were directed at older Hispanic pedestrians.

5. The effects of the three-year program were also examined with respect to gender and time of day. These analyses showed mixed results, with generally greater reductions in crashes for males and during daylight hours (between 10 a.m. and 6 p.m.) when compared to Broward County crashes.

\section{Zone-by-Zone Crash Evaluation Results}

In addition to the countywide crash analysis, the project team evaluated changes in pedestrian crashes in each of the high-crash zones that were targeted for countermeasure implementation.

For the zone analysis, numbers of pedestrian crashes (not crash rates) were used. Since no untreated control sites were available for this analysis, the resulting crash effects are less precise 
than if acceptable control zones had been available. This analysis was primarily intended to document the trends in pedestrian crashes for the specific pedestrian age and ethnic groups which were the targets of the countermeasures in those zones. Nonparametric tests (e.g., MannWhitney-U tests) were used for statistical significance testing, since the data were not normally distributed. The major findings appear in Table 33 and are summarized below:

1. Pedestrian crash frequency in Liberty City and South Beach decreased significantly for all pedestrian crashes from pre-program period to post-program period, while Little Haiti and Little Havana showed no significant changes in overall monthly crash frequency. Results of the tests for statistical significance are shown in Table 33.

Table 2. Mean Difference in Monthly Crashes From Before Period to After Period.

\begin{tabular}{|c|c|c|c|c|c|c|c|c|}
\hline \multirow[b]{2}{*}{ Age Group } & \multirow[b]{2}{*}{ Crash Zone } & \multicolumn{2}{|c|}{$\begin{array}{c}\text { Pre-Program } \\
\text { Period } \\
(1996 / 01-2002 / 01) \\
\end{array}$} & \multicolumn{2}{|c|}{$\begin{array}{c}\text { Post-Program } \\
\text { Period } \\
(2002 / 02-2004 / 12) \\
\end{array}$} & \multirow{2}{*}{$\begin{array}{c}\text { Mean } \\
\text { Diff. }\end{array}$} & \multirow{2}{*}{$\begin{array}{l}\text { T-TEST } \\
\text { p-value }\end{array}$} & \multirow{2}{*}{$\begin{array}{c}\text { Mann- } \\
\text { Whitney } \\
\text { U-TEST } \\
\text { p-value }\end{array}$} \\
\hline & & Mean & Std. Dev. & Mean & Std. Dev. & & & \\
\hline \multirow[t]{4}{*}{ Total } & Liberty City & 10.21 & 3.68 & 7.60 & 2.70 & -2.605 & $0.000^{*}$ & $0.000 *$ \\
\hline & Little Haiti & 4.77 & 1.95 & 4.71 & 2.55 & -0.053 & 0.905 & 0.798 \\
\hline & $\begin{array}{l}\text { Little } \\
\text { Havana }\end{array}$ & 6.60 & 3.01 & 6.89 & 2.45 & 0.283 & 0.629 & 0.476 \\
\hline & South Beach & 8.29 & 2.97 & 6.46 & 2.80 & -1.831 & $0.003 *$ & $0.004 *$ \\
\hline \multirow{4}{*}{$\begin{array}{l}\text { Child } \\
\text { Pedestrian } \\
(1-13)\end{array}$} & Liberty City & 2.89 & 1.81 & 2.11 & 1.32 & -0.776 & $0.026^{*}$ & $0.023 *$ \\
\hline & Little Haiti & 1.30 & 1.15 & 0.83 & 0.89 & -0.473 & $0.035^{*}$ & $0.047^{*}$ \\
\hline & $\begin{array}{l}\text { Little } \\
\text { Havana }\end{array}$ & 0.68 & 0.80 & 0.43 & 0.61 & -0.256 & 0.096 & 0.125 \\
\hline & South Beach & 0.29 & 0.51 & 0.11 & 0.32 & -0.173 & 0.070 & 0.079 \\
\hline \multirow{4}{*}{$\begin{array}{l}\text { School-Age } \\
\text { Child } \\
\text { Pedestrians } \\
(5-12)\end{array}$} & Liberty City & 2.18 & 1.51 & 1.37 & 0.97 & -0.807 & $0.005^{*}$ & $0.003^{*}$ \\
\hline & Little Haiti & 0.96 & 0.99 & 0.66 & 0.72 & -0.302 & 0.112 & 0.182 \\
\hline & $\begin{array}{l}\text { Little } \\
\text { Havana }\end{array}$ & 0.48 & 0.71 & 0.31 & 0.53 & -0.165 & 0.224 & 0.308 \\
\hline & South Beach & 0.22 & 0.45 & 0.09 & 0.28 & -0.133 & 0.110 & 0.116 \\
\hline \multirow{4}{*}{$\begin{array}{l}\text { Adult } \\
\text { Pedestrian } \\
(14-64)\end{array}$} & Liberty City & 5.90 & 2.28 & 4.89 & 2.62 & -1.018 & $0.041^{*}$ & $0.026^{*}$ \\
\hline & Little Haiti & 2.74 & 1.69 & 3.00 & 1.97 & 0.260 & 0.480 & 0.543 \\
\hline & $\begin{array}{l}\text { Little } \\
\text { Havana }\end{array}$ & 3.60 & 2.09 & 3.91 & 1.65 & 0.312 & 0.441 & 0.297 \\
\hline & South Beach & 6.26 & 2.48 & 4.80 & 2.21 & -1.460 & $0.004 *$ & $0.007 *$ \\
\hline \multirow{4}{*}{$\begin{array}{l}\text { Old } \\
\text { Pedestrian } \\
(>=65)\end{array}$} & Liberty City & 0.68 & 0.80 & 0.43 & 0.61 & -0.256 & 0.096 & 0.125 \\
\hline & Little Haiti & 0.41 & 0.57 & 0.34 & 0.68 & -0.068 & 0.589 & 0.305 \\
\hline & $\begin{array}{l}\text { Little } \\
\text { Havana }\end{array}$ & 1.73 & 1.71 & 2.11 & 1.41 & 0.388 & 0.246 & 0.080 \\
\hline & South Beach & 1.36 & 1.23 & 1.34 & 1.19 & -0.013 & 0.958 & 0.989 \\
\hline
\end{tabular}

2. For crashes involving school-age (age 5 to 12 ) child pedestrians, only Liberty City experienced significant decreases from the pre-program period to the post-program period. Liberty City, which had been identified as having the highest concentration of child pedestrian crashes in the pre-treatment period, experienced the greatest absolute reduction in child pedestrian crashes after the pedestrian safety program was 
implemented. For the four zones combined, there was an overall decrease of child pedestrian crashes from 3.84 per month ( 46 per year) to 2.43 per month ( 29 per year), a reduction of about $37 \%$.

The child pedestrian safety education program "WalkSafe" was initially implemented at all of the schools in Liberty City and was next implemented in Little Haiti, and then to approximately half of the 200 elementary schools throughout Miami-Dade County. Therefore, one might expect that any effect on reduced child pedestrian crashes would be more pronounced in those zones (i.e., Liberty City and Little Haiti) where the education programs began sooner and were also most intense. In fact, the largest absolute reductions in child pedestrian crashes occurred in these two zones.

3. In terms of crashes involving adult pedestrians (age 14 to 64), no significant changes were found in Little Haiti and Little Havana. From the pre-program to post-program periods, both Liberty City (17.2\% reduction) and South Beach (23.3\% reduction) experienced a significant drop in the number of crashes involving adult pedestrians. The countywide decrease was not statistically significant. The comprehensive pedestrian safety program consisted of a variety of treatments directed at different age groups and ethnic populations. To help to better understand these results, it should be remembered that some of these countermeasures (e.g., posters on transit vehicles) were directed at adult pedestrians in each of these four zones and to a lesser extent, in other parts of the county. South Beach was the zone that received a more extensive amount of pedestrian countermeasures (including being the only zone which received the special police safety enforcement program during the implementation period), which helps to explain why that zone experienced a significant reduction in crashes to pedestrians in the 14-to-64-yearold age group.

4. With respect to older pedestrians ( 65 and older), there was not a significant decline in crashes in Liberty City, Little Haiti, or South Beach. In Little Havana, there was actually an increase in older-pedestrian crashes. These results indicate that the pedestrian safety treatments directed at older adults (e.g., mostly safety education material and radio and TV PSAs) did not have the intended effect of reducing crashes involving older pedestrians.

\section{Discussion and Lessons Learned}

The study reveals that the combined Miami-Dade pedestrian safety program was associated with a significant reduction in pedestrian crashes countywide, and particularly among adult and child pedestrians within certain focus zones.

Additionally, the process of targeting countermeasures to specific age and ethnic groups appears to have been particularly successful in Liberty City and South Beach. Liberty City was the zone that received the most intense pedestrian safety education programs in all of its elementary schools, and child pedestrian crashes experienced greater absolute crash reductions compared to other zones and proportionally higher than countywide. After the pedestrian safety program, child pedestrian crashes decreased by $32.6 \%$ in the four targeted zones, and decreased by $22.1 \%$ 
countywide. These reductions agree closely with the reduction of approximately 20 to $30 \%$ in child pedestrian crashes due to "Willy Whistle" and the "And Keep on Looking" educational programs conducted in cities with comparable size and evaluated by NHTSA in the 1980s and 1990s.

Of the four zones targeted for specific countermeasures, South Beach was the recipient of the most intense amount of countermeasures, including selective police enforcement, a variety of educational and media messages, as well as a few engineering treatments. It is, therefore, encouraging that South Beach was found to be associated with a substantial reduction in pedestrian crashes $(22 \%)$ along with a $25.6 \%$ reduction in Liberty City.

However, not all the countermeasures were successful in reducing targeted crash types in all of the identified high-crash zones. Most notably, a variety of educational countermeasures in English and Spanish were implemented in Little Havana, where there had been a high prevalence of crashes involving older, Spanish-speaking pedestrians. Countermeasures included the distribution of educational material at senior centers, safety education meetings, television and radio messages, and other education measures. In spite of these efforts, there was no significant reduction in crashes involving older pedestrians or involving pedestrians in general in Little Havana as a result of the countermeasures implemented there. The reasons for the lack of success of the program in Little Havana are not known. Likewise, no significant reductions in pedestrian crashes resulted in Little Haiti. Such findings may provide some understanding about what might be expected from similar pedestrian safety programs, and perhaps how to address more challenging crash problems, such as crashes involving older pedestrians.

The greater reduction in pedestrian crashes that resulted in the targeted zones in Miami-Dade County was consistent with similar findings from the previous crash zone studies for NHTSA. In other words, the greatest reduction in pedestrian crashes occurred in the zones where countermeasure implementation was most extensive. Certainly more intensive education (with enforcement and engineering) treatments may be needed to have a clear reduction in senior pedestrian crashes.

Additional lessons learned include the importance of quality GIS data in identifying problem locations and sub-populations, quantifying specific problem types, evaluating results, and communicating the issues of pedestrian safety to enlist the support of relevant agencies; the importance of interagency relationships; and the benefits of institutionalization of a comprehensive pedestrian safety program. 


\section{CHAPTER 1: INTRODUCTION}

\section{Background}

The number of pedestrians killed in United States traffic crashes has declined over $40 \%$ since peaking in the late 1970s and early 1980s. Still, in 2005 there were 4,881 recorded pedestrian fatalities, representing $11 \%$ of all U.S. traffic deaths (NHTSA, 2006, Table 53). In urban areas where pedestrian activity and traffic volumes are greater compared to rural areas, pedestrians often comprise a much larger portion of traffic deaths. In 2005, 47\% of New York City's traffic deaths were pedestrians; and in 27 other urban areas with populations greater than 150,000, pedestrians comprised 30\% or more of total traffic fatalities (NHTSA, 2006, Table 121).

Pedestrian safety has been a focus of NHTSA's efforts to save lives and reduce injuries since the agency was established nearly four decades ago. During the 1970s, a research project series was carried out to identify the causal factors of pedestrian crashes and appropriate countermeasures to address these causes. The initial research by Snyder and Knoblauch (1971) focused on urban pedestrian crashes, but subsequent studies extended the methodology to rural crashes and crashes occurring on freeways (see Stutts et al., 1992, for a review). From this research evolved the basic pedestrian crash typology that remains a cornerstone of much of NHTSA's pedestrian safety activity, as well as that of the Federal Highway Administration (FHWA). Crash types describe behaviorally similar pre-crash actions that lead to characteristic pedestrian-motor-vehiclecollision situations (see Table 3 for examples of common crash types and descriptions). A more complete list of crash types and combinations used in the analysis is provided in Appendix A.

Table 3. Common Crash Types and Descriptions.

\begin{tabular}{|c|c|c|}
\hline $\begin{array}{c}\text { Crash } \\
\text { Type }\end{array}$ & Description* & Image \\
\hline $\begin{array}{l}\text { Midblock } \\
\text { Dash }\end{array}$ & $\begin{array}{l}\text { At a midblock location, the pedestrian } \\
\text { was struck after entering roadway and } \\
\text { the motorist's view of the pedestrian } \\
\text { was not obstructed. }\end{array}$ & \\
\hline $\begin{array}{l}\text { Intersection } \\
\text { Dash }\end{array}$ & $\begin{array}{l}\text { The motorist's view of the pedestrian } \\
\text { was blocked until an instant before } \\
\text { impact and /or the pedestrian was } \\
\text { struck while running. }\end{array}$ & \\
\hline
\end{tabular}




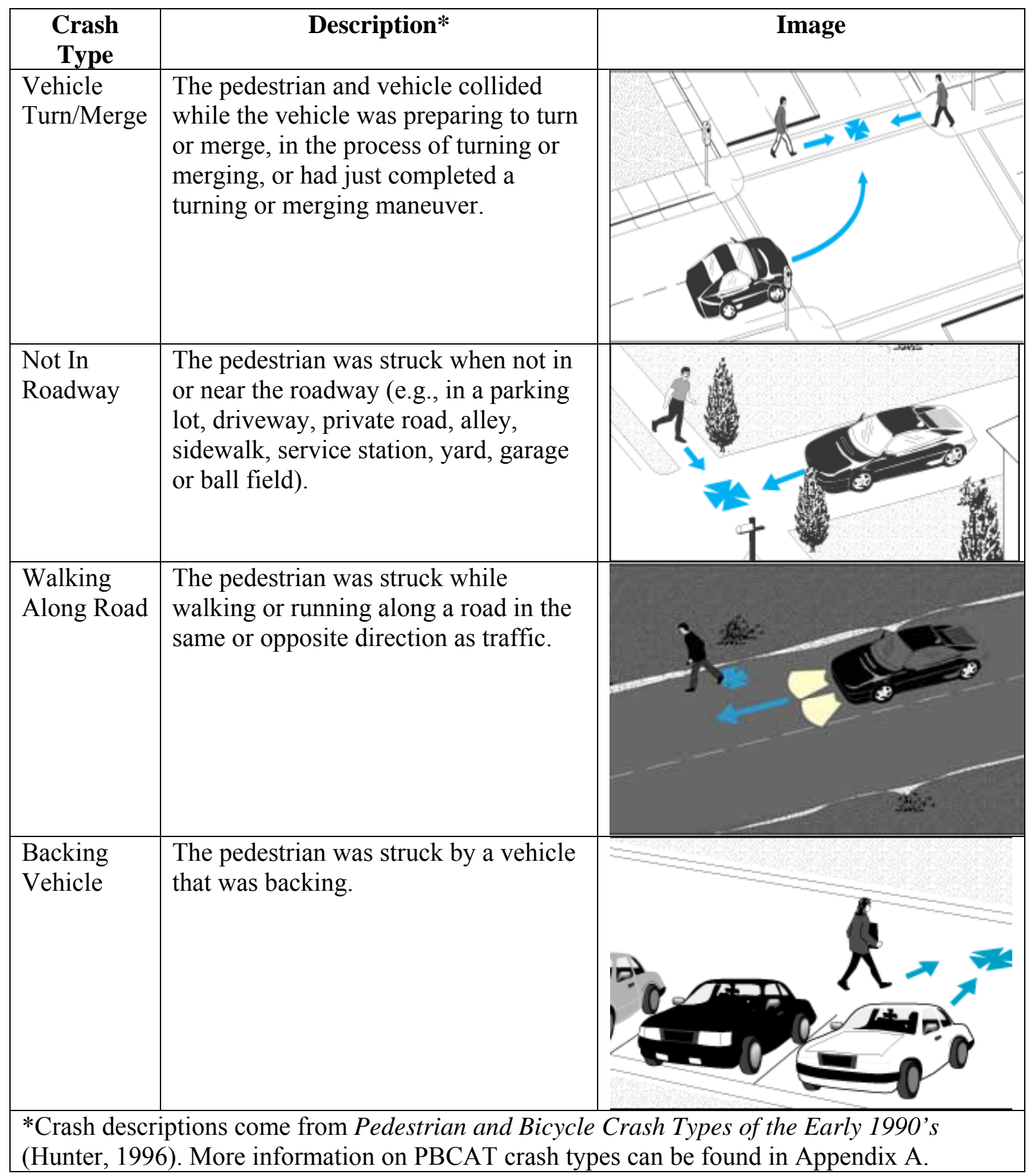

The aim in typing crashes is to gain a better understanding of the environmental correlations to crashes and behavioral errors on the part of drivers and pedestrians, so that appropriate countermeasures can be developed. The recent upgrade of the Pedestrian and Bicycle Crash Analysis Tool (PBCAT, v. 2.0), an automated typing software program developed for FHWA, includes 56 distinct crash types that may be classed into 16 groups (Harkey et al., 2006). FHWA also recently published PEDSAFE: Pedestrian Safety Guide and Countermeasure Selection System (Harkey \& Zegeer, 2004). The interactive online guide is designed to assist local 
engineers, planners, and other safety professionals in selecting the most appropriate countermeasures for addressing their particular crash types or a more general pedestrian safety objective. It includes a wide range of engineering as well as education and enforcement/regulatory tools tailored to specific crash types and locations.

During the late 1990s, NHTSA also developed the concept of pedestrian safety zones. The idea behind pedestrian safety zones is to focus improvements where the problem is greatest. By concentrating efforts where the majority of the problem or the target audience exists, funds are used more efficiently and activities that would be prohibitively expensive if applied to an entire community can be applied to greatest benefit on a smaller scale (Blomberg \& Cleven, 1998).

To apply the pedestrian safety zone approach, one first identifies the crash population of interest (school age children, older adults, impaired pedestrians, ethnic minorities, etc.). Then, pedestrian crashes involving this population are plotted onto a map of the community, either manually or using a computer-based geographic information system (GIS). Once crashes are mapped, potential zones are identified by searching for clusters of events. These may be circular areas (e.g., defined by a one-mile radius from a given point), linear zones (e.g., along a roadway segment), or irregularly shaped areas that reflect natural boundaries within the community (e.g., a Hispanic community). The goal is to identify an area where the ratio of the percentage of the problem addressed to the percentage of land area covered is 3:1 or greater. Efforts are then focused on identifying needs within this zone, along with resources and countermeasures for addressing these needs. The program is implemented, and activities are monitored and evaluated. Over the course of a long-term pedestrian safety initiative, old zones may be discontinued or new zones established. (See Blomberg \& Cleven, 1998, for a more detailed description of the approach.)

The project draws heavily upon previous NHTSA and FHWA research to identify and evaluate countermeasures for improving pedestrian safety, and applies this knowledge on a broad scale to produce tangible, community-wide safety benefits. A focus of the current demonstration project was to extend application of the pedestrian safety zone approach to a large urban area and to document and evaluate the process so that it can be replicated in other metropolitan areas afflicted by high numbers of pedestrian deaths and injuries.

\section{Project Goals and Objectives}

The overall goal of the project was to reduce deaths and injuries to pedestrians in a large urban environment by implementing a long-term pedestrian safety program within the jurisdiction. The specific project objectives were to work with stakeholders in the chosen community to:

1. Obtain and analyze pedestrian crash data to identify zones of high incidence of pedestrian crashes and the special characteristics of those crashes;

2. Identify and implement a comprehensive program of education, engineering, and enforcement strategies to address the problems identified;

3. Evaluate the safety benefits of the program; and

4. Document the process and prepare a guidance document for other cities or urban areas that may want to replicate the process. 


\section{Project Site}

The project was conducted in Miami-Dade County (formerly called Dade County). This location was selected because of the significant pedestrian injury and fatality problems the area was experiencing. In 2001, just before the project began, Florida was the fourth largest State in terms of population (16.4 million), but ranked first in number of pedestrian fatalities with 489 .

California had 711 pedestrian fatalities but had more than double (34.5 million people) the population of Florida. In terms of pedestrian fatality rate (fatalities per 100,000 population), Florida again ranked near the top at 2.98, behind only New Mexico (3.94) and Arizona (3.00). In Florida, with its experience of a high number of pedestrian fatalities, Dade County (in 2001) led the State in pedestrian deaths and injuries.

Miami-Dade County encompasses nearly 2,400 square miles (larger than Rhode Island and nearly equal to the land mass of Delaware), with one-third of this area lying within Everglades National Park. Its estimated population in 1994 was nearly 2 million people; by 2005 it was nearly 2.4 million people, making it the eighth most populous county in the United States. The county is home to the City of Miami and 34 other distinct jurisdictions. The Miami-Dade County government has responsibility for all transportation operations and improvements within the area, and works closely with the City of Miami and other local officials (see Figure 1 and Figure 4).

The area has a diverse population: it has a significant retirement population; it is a main tourist attraction and a home to large minority populations, the most predominant being Hispanic. When the project started, the county population was approximately $50 \%$ Hispanic, $20 \%$ non-Hispanic Black and 30\% non-Hispanic White.

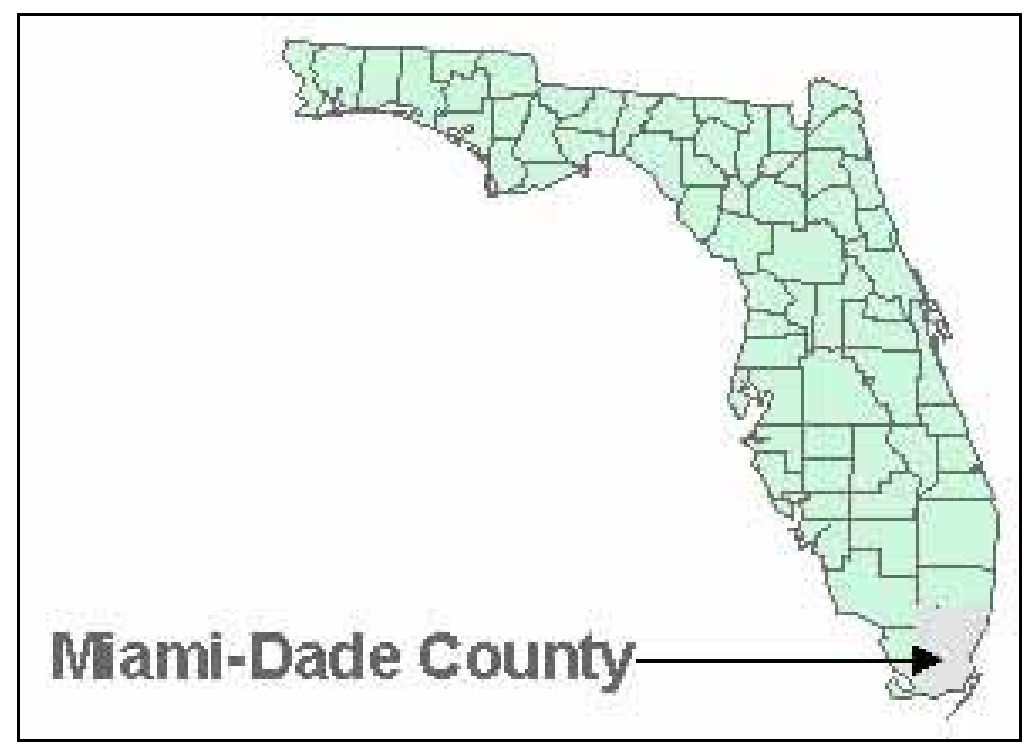

Figure 3. Map of Miami-Dade County, FL. 


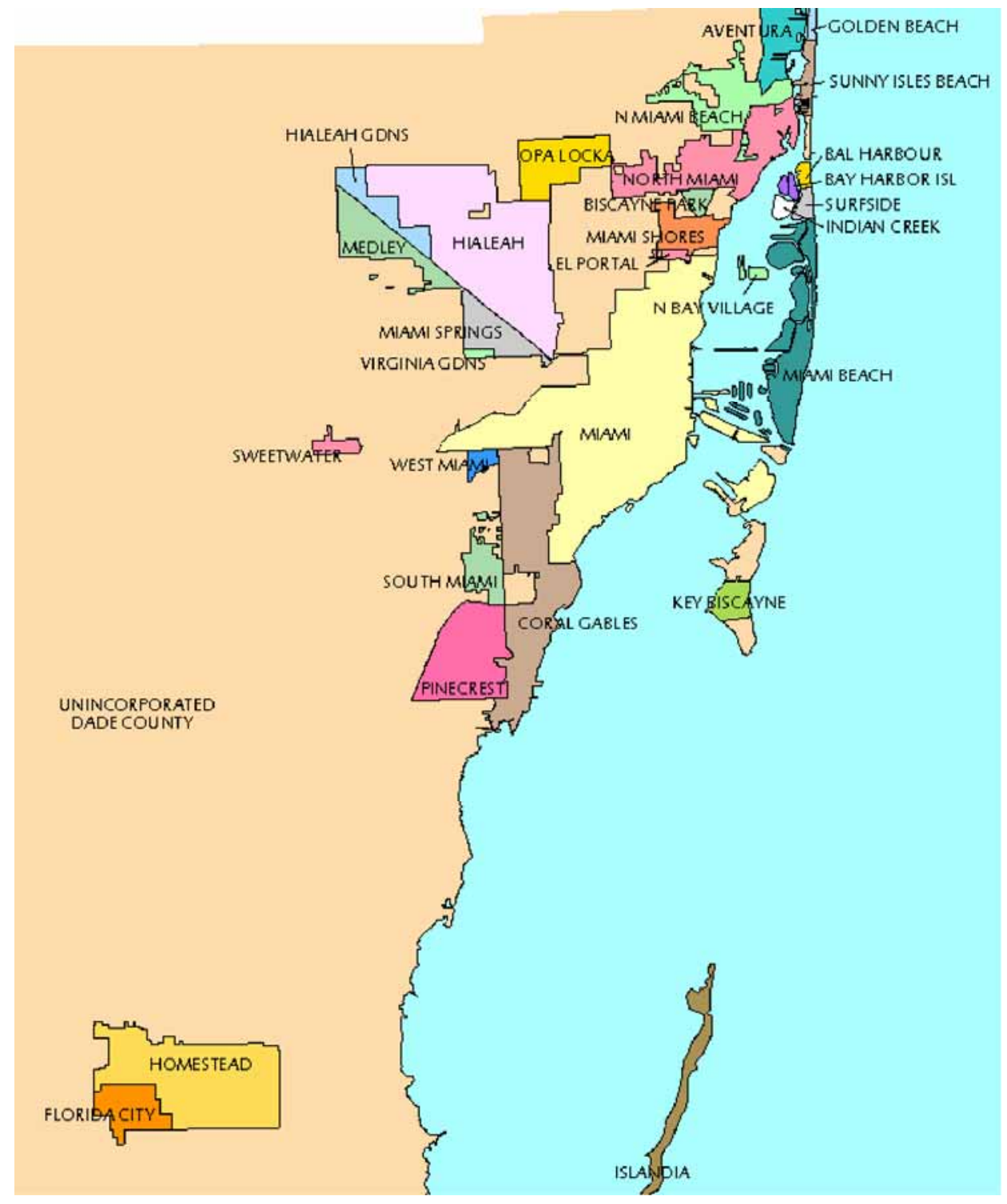

Figure 4. Miami-Dade County Municipalities.

In addition to its large urban population and significant pedestrian safety problem, there were a number of other reasons that led to the selection of Miami-Dade as the site for the proposed pedestrian demonstration project:

- A sufficiently large and diverse area to support multiple, simultaneous countermeasure implementations;

- Excellent sources of available data to assist in defining the area's pedestrian safety problems, including GIS data for pinpointing pedestrian crash locations, crash report data already being gathered by the county's pedestrian-bicycle coordinator, access to trauma center data, as well as a willingness on the part of local partners to collect observational behavior data; 
- Multidisciplinary interest among the Miami-Dade departments and agencies, necessary to implement a successful countermeasure demonstration;

- Support at the State level for developing countermeasures to address a significant portion of Florida's pedestrian crash problem;

- Strong pedestrian leadership both in the county and at the State level committed to an enduring pedestrian safety effort in the County;

- Familiarity of project staff with the area; and

- A full range of crash types and target groups - including children, seniors, a substantial minority population, etc. - that contribute to a strong prototype project.

\section{General Approach and Project Roles}

The overall approach to conducting the demonstration project was for the University of North Carolina's Highway Safety Research Center (HSRC) and staff of Dunlap and Associates, Inc., Stamford, Connecticut, to provide technical expertise and support to the project, but for a coalition of local leaders and stakeholders to oversee the selection and implementation of countermeasures as well as collection of crash and other data for evaluating the program's effectiveness. Relying on local resources and funding to implement the program was considered key to the program's long term sustainability, as well as its potential as a model for other cities.

Specifically, HSRC/Dunlap provided technical expertise in the collection and analysis of pedestrian crash data and identification of pedestrian safety zones and target populations. Project staff also provided a menu of education, enforcement, engineering, and environmental strategies for consideration by the community task force, along with actual education and awareness materials for use by the program. The project supported an $\$ 80,000$ grant to augment local dedicated personnel time for the pedestrian initiative, and staff assisted in identifying additional resources for the program, including State funding (e.g., to install engineering treatments). Members of the project team were also responsible for the overall evaluation of the project. Throughout the project period, FDOT provided additional funding directly to the Miami-Dade Metropolitan Planning Organization (MPO) to help pay for the data preparation (geocoding) process for the nine years of pedestrian crash data and also to provide additional funding for a wide variety of educational and engineering countermeasures.

For its part, Miami-Dade staff committed to making pedestrian safety a high, ongoing priority for the county; established a local pedestrian safety task force to focus attention on the problem; provided a person (David Henderson, the Miami-Dade MPO pedestrian-bicycle coordinator) to spearhead the local effort; assisted in the data collection required for planning the program and conducting the evaluation; and was primarily responsible for developing and implementing the multiyear pedestrian safety program.

Together, members of the project team conducted a detailed analysis of Miami-Dade's pedestrian crash problem for the years 1996 to 2001, using available geocoding and crash typing tools. This led to the identification of four high-incident pedestrian crash zones, or target areas for intervention. Under the direction of the task force, countermeasures were developed and implemented in each of the target areas over a period of several years. The existing Miami-Dade Injury Prevention Coalition and the Safe Kids Coalition were used in lieu of a separate, free- 
standing task force for the project. This allowed for better local participation and help with institutionalizing the program after the project was completed. The various organizations that participated in this coalition-based task force included members from the Jackson Memorial Medical Center, Miami Children's Hospital, Florida Department of Health, Miami-Dade County Health Department, Lehman Injury Research Center, Miami-Dade Fire Rescue, county and local police departments, the Florida Department of Transportation, Miami-Dade County Public Schools, Ryder Trauma Center, and others. Program activities were documented, and the overall effectiveness of the efforts evaluated using time series and other data analysis techniques. The entire project spanned almost nine years, from October 1998 through March 2007.

\section{Report Organization}

This report documents the results of this multiyear pedestrian safety demonstration and evaluation project. Chapter 2 summarizes past research and related programs. Chapter 3 describes the project methodology in greater detail, including the creation of local partnerships, the analysis of the problem, and the development of databases for evaluating the project's impact. Chapter 4 describes the countermeasures selected and implemented. The results of the project are documented in Chapter 5 and include both process outcomes (activities implemented, other projects spawned, etc.) and results from the analysis of the pedestrian crash databases. A final discussion in Chapter 6 highlights the project's accomplishments and limitations, and provides guidance on extending the project approach to other cities. 


\section{CHAPTER 2: PAST RESEARCH AND RELATED PROGRAMS}

The study team drew from prior research and program efforts developed by NHTSA, FHWA, and State and local agencies to identify appropriate safety messages, educational programs and materials, engineering, and enforcement countermeasures to address the problems identified (see Chapter 3). Following is a summary of the relevant research and program evaluations that provided a basis for development of the Miami-Dade countermeasures and strategies. Some of the discussion is drawn from Cleven and Blomberg (2007).

Crash data in the 1970s indicated that a high percentage of child pedestrian crashes occurred in local neighborhoods. Typical crashes involved young children darting out into the street, often from between parked cars, without first stopping at the curb and adequately looking for oncoming traffic. Early NHTSA research carried out by Blomberg and colleagues developed, produced, and field-tested three sets of pedestrian safety public information and education (PI\&E) messages using "Willy Whistle" to target child pedestrians (Blomberg et al., 1983). Willy Whistle is an animated police officer's whistle that teaches children age 4 to 7 how to stop and look before crossing the street and safely conduct a midblock crossing. Using print, television, and the Willy Whistle film to convey messages stressing anti-dart-out behavior a significant reduction was found in the number of dart-out pedestrian crashes involving 4- to 6year-old children by approximately $30 \%$ in the three test cities of Los Angeles, California; Columbus, Ohio; and Milwaukee, Wisconsin.

A second film titled "And Keep on Looking," targeting children in $4^{\text {th }}$ through $7^{\text {th }}$ grades, builds upon Willy Whistle by addressing more complex pedestrian traffic situations, e.g., at intersection locations. Training including looking out for turning vehicles and the meaning of traffic signals. An evaluation of the film's efficacy showed an increase in safe street crossing knowledge for children age 9 to 12 in Seattle, Washington, while an assessment in Milwaukee indicated a pedestrian crash reduction for children 9 to 12 of greater than $20 \%$ compared with children in areas surrounding Milwaukee and children in comparison cities (Preusser \& Lund, 1988). Both films, "And Keep on Looking" and "Willy Whistle" underwent updates in the early 1990s and were given new titles: "Stop and Look With Willy Whistle" and "Walking With Your Eyes" (Stutts et al., 1992, pg 28-29). Each of these films was used in the WalkSafe program in MiamiDade County.

Research examined pedestrian crashes occurring to adults. One common crash type involved vehicles that were turning and merging into traffic. Advice given to pedestrians included the need to stop and actively search for turning vehicles since drivers might miss them while turning. Another adult crash type, called the multiple threat, involves multilane situations in which a driver in the closest lane to them stops but other vehicles in adjacent lanes do not. Advice given to pedestrians included where to stop - at the stopped vehicle's edge line - and look for oncoming traffic. Radio and television spots were used to convey adult safety messages about vehicle turn/merge and multiple threat situations in a field study in Los Angeles and San Diego. The adult messages yielded positive results, especially among Spanish-speaking adult pedestrians (Blomberg et al., 1983). Overall, the public information and education messages for children and adults demonstrated that public information and education alone can, in general, be 
successful in reducing crashes if the target audience receives adequate exposure (Blomberg et al., 1983).

In the early 1980s, efforts continued into understanding and developing countermeasures to target contributing factors, crash types, and specific populations. Hale and Zeidler (1984) reviewed the literature and programs for pedestrian and bicyclist conspicuity. Researchers also conducted field tests to assess the effectiveness of various strategies and material in enhancing nighttime conspicuity (Blomberg, Hale, \& Preusser, 1984). The classic recommendation to wear white clothing was considered insufficient to provide a suitable level of detection and safety. Recommendations based on results of the study included carrying or using an active light source supplemented by retro-reflective materials, particularly arm and leg bands that highlight the shape and movement of pedestrians.

Older pedestrians are overrepresented in fatal crashes. In 2005 there were 981 fatalities among people 65 and older. Although older people represent only $12.4 \%$ of the population, older pedestrians represent $20 \%$ of all 4881 pedestrian fatalities. Blomberg, Cleven, and Edwards (1993) analyzed several pedestrian crash datasets and identified vehicle turns/merges, other intersection crashes, and backing vehicles as crash type groups, and identified conspicuity as an additional factor to be targeted with countermeasures. Through an iterative process involving five discussion groups comprised of older adults, appropriate pedestrian safety countermeasure messages were developed for each of the four defined situations. The risk and behavioral advice were documented in the background paper Walking Through the Years, which was used to create a multipage education brochure that could be directly distributed to older target audiences, titled Walking Through the Years: Pedestrian Safety for the Older Adult (65+) (Blomberg et al., 1993). Additionally, a slide series and accompanying presenters guide were prepared for summarizing study efforts for potential distribution to organizations and training groups of older pedestrians. Blomberg and colleagues' (1993) research also determined that the best media distribution plan for the pedestrian safety risk and behavioral advice was to place primary emphasis on getting groups which already had open communication channels with older populations to act as primary disseminators such as AARP (formerly the American Association of Retired Persons), AAA (formerly the American Automobile Association), and the National Safety Council (NSC).

Blomberg and Cleven (1998) developed and applied a procedure for defining pedestrian safety zones, the approach used in the present study, in a program for older adult pedestrians $(65+)$ in Phoenix, Arizona, and Chicago, Illinois. Once the safety zones had been identified, a pedestrian crash countermeasure program was developed, implemented, and evaluated within the defined zones. A combination of public education and engineering countermeasures was delivered to the older adults within each zone. The countermeasures included education countermeasures: a comprehensive video; multiple television and radio public service announcements; 13 information fliers; placards on buses; and engineering countermeasures including the installation of rumble strips near high-use crosswalks as well as better pedestrian signs, removal of objects that impeded motorist and pedestrian sight distance at intersections, and appropriate crosswalk treatments. The evaluation, which was conducted only for Phoenix, showed a significant reduction in crashes to pedestrians 65 and older within the zones, while the city experienced a increase in overall population and pedestrian crashes involving people under 65 during the same time period. Moreover, the study concluded that identifying zones was an effective and 
economically efficient means of deploying pedestrian countermeasures. One of the most successful countermeasures in the study involved the delivery of fliers to each residence in the safety zones at an approximate cost of $\$ 24,000$. Had the flier delivery gone to each residence in the entire city, the cost would have been 12 times that amount (Blomberg \& Cleven, 1998).

A 1995 study by Hunter and colleagues used the NHTSA typing methodology to type more than 5,000 pedestrian collisions in six States (Hunter et al., 1996). More than three-fourths of the crashes fell into one of eight crash-type categories, including midblock and intersection dash/ dart-outs; other midblock and intersection crash types; motor vehicle turns/merges; walking along the roadway; and backing-vehicle crashes. Other factors associated with collisions or injury severity included pedestrian age, time of day, and roadway factors such as speed limit, number of lanes, and type of location (intersection, mid-block, off-roadway). This crash factor and topology information allowed for the development of specific interventions, including a mixture of education and information, enforcement and engineering countermeasures.

\section{Review of Comprehensive Community-Wide Programs}

Numerous cities have integrated education, enforcement, and engineering countermeasures into their pedestrian safety programs. Two examples are Denver, Colorado, and Seattle, Washington. From 1977 to 1980, the Denver Pedestrian Safety Project integrated efforts from various organizations such as the Colorado Division of Highway Safety, Denver Police Department, and engineers and researchers from Applied Science Associates, Inc., to identify specific pedestrian crash problems and develop, implement, and evaluate appropriate countermeasures. These countermeasures ranged from pedestrian law enforcement at high-crash locations and public information and child education programs to traffic engineering modifications for pedestrians including a pedestrian mall and pedestrian trails. These combined efforts of the Denver program were associated with significant reductions in pedestrian collisions in Denver, compared to increases for three comparison cities over the same time period (see review in Stutts et al., 1992). The Denver Pedestrian Safety Project was an excellent example of a program using a coordinated community approach to reducing pedestrian crashes (Thackray \& Chiplock, 1981).

Pedestrian safety activities in Seattle incorporated education, engineering, and enforcement activities to reduce the number of pedestrian crashes and injuries. The main focus of the educational activities was on children and included parent-child activity books, information fliers and posters, pedestrian rodeos, information from school PTAs, and the use of "Kids and Cars Don't Mix," a school safety curriculum. The latter uses videos, worksheets, safety rap songs, posters, and other props to teach children safe street-crossing behaviors (Stutts et al., 1992). An information campaign targeting the general public included public service announcements on television, radio, and billboards, as well as announcements in local newspapers and posters on buses.

Engineering countermeasure activities involved the Seattle Engineering Department, which participated in identifying traffic situations that pose the least risk to pedestrians. Other engineering changes included installing various traffic-calming devices, improving lighting, and constructing a "priority accessible network" for older and handicapped pedestrians (Stutts et al., 1992). 
To heighten awareness of motorists' need to yield to pedestrians, one component of the enforcement countermeasures involved issuing citations to jaywalkers and to motorists failing to yield to pedestrians in crosswalks. The latter included placing pedestrian decoys at crosswalks with police officers ticketing motorists who failed to yield to the pedestrian. This practice was supported by the general public and even used citizen volunteers to serve as decoys (Stutts et al, . 1992). A 2003 study by Van Houten and Malenfant evaluated a safety enforcement effort targeted at motorists who fail to stop for pedestrians at four marked crosswalks on high-crash corridors in Miami Beach, Florida. Police stopped 1,562 motorists over a one-year period, with 307 citations given. The percentage of motorists yielding to pedestrians increased significantly at the crosswalks that received enforcement, while motorist yielding remained unchanged at the untreated control sites. This enforcement effort and analysis were conducted in conjunction with the Miami-Dade Pedestrian Safety Demonstration Study, and more details are provided later in this report. 


\section{CHAPTER 3: STUDY METHODOLOGY}

\section{Local Partnership Development}

In order to promote a sustainable program and capitalize on existing partnerships and knowledge, the existing network of safety organizations in Miami-Dade County was used to coordinate the project activities. At the start of the project, the monthly meetings of the Miami-Dade Safe Kids Coalition (coordinated through the Miami Children's Hospital) and the Miami-Dade Injury Prevention Coalition (hosted by Jackson Memorial Hospital) were used to disseminate information about the project, the pedestrian crash data, the countermeasures being implemented, and to get feedback. Membership of the Safe Kids Coalition and Injury Prevention included law enforcement, State and county health departments, Jackson Memorial and Miami Children's Hospitals, and other organizations with an interest in safety. The safety events coordinated by these coalitions were also used to distribute pedestrian safety material to the public. During the project, FDOT began to implement the Community Traffic Safety Program that resulted in the creation of several Community Traffic Safety Teams within Miami-Dade County, organized around municipal police departments. These teams were also involved in implementing pedestrian safety countermeasures, including Walk to School Day and targeted enforcement activities focusing on red-light-running, seat belt usage, and drunk driving.

Walk to School Day is a major activity of the Safe Kids Coalition, which used the project data to target schools in areas with large numbers of juvenile pedestrian traffic crashes. The local coalition works with participating schools to distribute safety and education resources provided by the parent organization, Safe Kids Worldwide. The Miami-Dade coalition also used resources from the project in schools in the target zones.

The Miami-Dade Injury Prevention Coalition (IPC) aims to address preventable injuries to people 14 and older. The traffic safety efforts of the IPC had previously focused on increasing seat belt usage and not on adult pedestrian safety; however, for the current project, pedestrian safety material was distributed through the members of the IPC.

In addition to these permanent safety groups, the University of Miami School of Medicine organized the WalkSafe Program Task Force to help promote and coordinate the pedestrian safety activities of the school-based education program, WalkSafe. The task force included staff from the Miami-Dade County Public Schools, Public Works, School of Medicine, and MPO to help develop and implement the WalkSafe educational program in elementary schools. Because of the difficulty of getting new programs introduced into classrooms it was important to have a high level of support and involvement (including a school board member) at the organizational stage of the project.

To obtain area-specific community input, additional meetings were held in target areas with the public and with local government representatives. The purpose of the meetings was to introduce the project and solicit information on the nature of the pedestrian safety problem and strategies for improvements. 
The use of existing committees and organizations to receive input and coordinate pedestrian safety efforts helped achieve the project goal of institutionalizing pedestrian safety within the community. Because most of the existing groups operated countywide (SafeKids, Miami-Dade Injury Prevention Coalition) or were created in areas with the resources to devote to safety (Community Traffic Safety Teams), there was no guarantee that the appropriate level of attention would be focused on the targeted high crash areas. However, the availability of the pedestrian crash database and GIS mapping software made it possible to illustrate the needs of the target areas in a way that members of the safety partnership could easily understand.

\section{Study Design and Data Sources}

The design for the Miami-Dade pedestrian demonstration included the use of pedestrian crashes as the sole outcome measure of effectiveness. While intermediate measures of pedestrian and driver behavior have been used in other evaluation studies of pedestrian countermeasures, they were not feasible in the current effort for at least two reasons. First, the interventions in the project were to be comprehensive and therefore would cover a multitude of potentially relevant behaviors, most of which would be difficult or costly to measure in a valid and reliable manner. Second, the available pedestrian crash data for Miami-Dade, both baseline and postinterventions, were sufficiently large to support a sensitive assessment of program success based on the ultimate crash reduction outcome measure.

Once the decision was made to focus only on crashes as the project's evaluation measure, effort turned to building a database of crashes to support analyses of effectiveness. This involved selecting a source for crash data, retrieving and coding the data, and defining appropriate subsets for analysis.

\section{Crash Data Source and Retrieval Process}

The standard source of highway crash data, including pedestrian crashes, is police crash reports (PCRs). These are typically completed by police officers or police aides at the scene of a crash or at a police station. Occasionally, PCRs are filed by involved parties without any intervention by a police officer.

Once a PCR is created, it is typically stored in at least two locations: the originating department and a State agency. Miami-Dade PCRs are generated by multiple police agencies (local police departments, the sheriff's office and the Florida Highway Patrol), each of which covers part of the county or a particular type of road, e.g., freeways. Thus, to access all PCRs for the county locally, liaison would be required with multiple organizations. On the other hand, all county PCRs are sent to the Department of Highway Safety and Motor Vehicles (DHSMV) in Tallahassee for archiving and analysis. DHSMV enters the individual reports into a computer database and then archives an image of each report that can be printed out at any time if someone requests a hard copy.

Since DHSMV had all Miami-Dade PCRs for the period of interest (1996 - 2004, inclusive), it was decided to use its files as the source of all crash data for analysis. Liaison was established with DHSMV, and it agreed to support the project and provide all required documentation on 
their crash files. Procedures were then developed to access the crash data and produce analysis files of interest.

\section{Data Collection and Processing}

The data that were used to identify high-crash areas and monitor the results of the project were developed by the MPO with assistance from the Florida DHSMV, the University of Florida Department of Urban and Regional Planning (UF-DURP), and the University of North Carolina Highway Safety Research Center (HSRC). The database of all Florida traffic crashes was obtained from the DHSMV and sent to HSRC, where it was processed to produce a single file of Miami-Dade County pedestrian crashes. This file contains all of the data contained in each traffic crash report except the crash location, sketch and narrative. The crash location, sketch, and narrative are only available from the hard copy of the report. To get the hard copies of each Miami-Dade pedestrian crash, the crash report numbers were taken from the database and used to create a "pull list." The pull list was sent to a temporary staffing agency in Tallahassee, where workers used the report numbers to find and copy the appropriate reports. The hard copies of the reports were then shipped out for crash typing and address matching. This involved processing approximately 1,600 to 1,800 pedestrian crash reports per year for nine years of crash data (1996-2004).

\section{Address Matching (Geocoding)}

Address matching is the process of locating the crash event within the electronic street map of a geographic information system (GIS). Using software developed by UF-DURP, the address data was formatted to increase the accuracy of the matching in ArcView GIS. The location of each pedestrian crash was entered manually and matched by the software or, if the software could not find the crash location, located on the street map by hand. Through the processes of crash typing and address matching, the database was refined by eliminating crash reports that were not traffic crashes involving pedestrians or located in Miami-Dade County.

\section{Description of the Study Databases}

The Florida DHSMV database used in the analysis consisted of all of the pedestrian-related crashes which were reported and coded into the electronic file. This was the original data source that was used for the evaluation purposes for the countywide crash evaluation. It was also used for creating the non-Miami-Dade control group databases, as well as the zone database. These are described below:

- Statewide Pedestrian Crash Data (Statewide Data) - Statewide data were accessed via a set of computer tapes provided by DHSMV containing all crash information for Florida. These were processed to determine whether a pedestrian was involved in the crash. The subset of all identified statewide pedestrian crashes was then extracted into an analysis file. This file was used to determine the trend of pedestrian crashes in Miami-Dade as well as the comparison of the Miami-Dade experience with the rest of the State and other comparable counties. This database was also used to produce suitable control groups of pedestrian crashes in Florida, as will be described later. 
- Miami-Dade DHSMV Pedestrian Crash Data (Miami-Dade Data) -A subset of the total State pedestrian crash file was created based on the county code stored in each record. This produced an analysis set with all crashes held by DHSMV that contained both a code indicating that a pedestrian was involved and a code indicating a location of occurrence in Miami-Dade. It was later determined while creating the zone subsets discussed below that some of the people coded as pedestrians were, in fact, bicyclists or other non-pedestrian crash participants. Also, some of the county codes were incorrect. Thus, it was decided that a filtered Miami-Dade database was needed in order to remove the crashes that clearly did not belong in an analysis set. This file was used to assess the trend over time in Miami-Dade crashes as archived by DHSMV.

- Crashes that Occurred within the Defined Zones (Zone Data) - The formation of this analysis subset required access to hard-copy PCRs for the Miami-Dade DHSMV pedestrian crash data. The DHSMV computer database does not include the specific location of a crash. Only the county and city of occurrence are part of the file. Thus, in order to determine which crashes took place within the zones, it was necessary to read each PCR to attempt to determine the precise location of the crash. The location could be determined for most of the reports. Once determined, it was entered into a geographic information system (GIS) program so that those crashes occurring in the zones could be identified. Crashes whose location could not be determined more precisely were included in the total Miami-Dade crash subset outside of the zones. Each crash was also assigned a crash type code from the NHTSA Manual Accident Typing System (MAT) for all years (1996 through 2004). Crashes from 2002 through 2004 also received a crash type in accordance with the Pedestrian and Bicycle Crash Analysis Tool, since the PBCAT tool was available for use in 2002. However, for consistency purposes, the analysis in this study made use of the MAT coding, since MAT codes were available for all years. This analysis file was used for sensitive time series analyses of crash changes over time in the defined zones. See Appendix A for information on PBCAT crash typing codes.

Therefore, this database was a filtered file of all Miami-Dade Pedestrian crashes which were geocoded and crash-typed based on all pedestrian crashes in the DHSMV computer files in Miami-Dade. Specifically, crashes not involving a pedestrian (mostly bicycle/motor vehicle crashes) and those outside of Miami-Dade were excluded. This yielded a file without misclassified crashes that was useful for examining the MiamiDade crash rate over time. ${ }^{1}$

\section{Data Sample}

Miami-Dade County has the highest incidence of pedestrian injuries and fatalities in Florida, which ranked first among the States in number of crashes as well as per capita pedestrian crashes in recent years. Over the nine years of the project period, there were 17,308 total pedestrian crashes in Miami-Dade County, which included 724 fatal crashes (4.2\%). After screening and geocoding all hard-copy crash reports, a total of 15,472 pedestrian-motor vehicle crashes

\footnotetext{
${ }^{1}$ It must be noted that this file is still missing Miami-Dade pedestrian crashes that were either miscoded as nonpedestrian events or assigned the wrong county code and therefore were not selected in the initial screen of records from the DHSMV crash tapes. All available information suggests that the number of Miami-Dade pedestrian crashes misclassified in this manner in the DHSMV database is likely small.
} 
remained, which were geocoded and used in the zone analysis. Crashes were dropped because they were out of the county, did not involve a pedestrian, or could not be geocoded. Fatal injuries were reported in 670 crashes, with 3,002 crashes involving serious injury. Table 4 shows the distribution of crashes over the nine-year study period in both of the databases used. As can be seen, there have been some up and down trends over the years of the study period; with an apparent downward trend in total crashes from 1996 to 1999, before the program countermeasures were implemented. Most of the countermeasures were implemented after January 2002, so the "before period" used in the analysis is 1996 through 2001, and the "after period" is 2002 through 2004. The year 2002 was selected as the first year of the after period, since some of the countermeasures were implemented near the beginning of 2002. Of course, more of the countermeasures were underway by 2003, so the combined program effects might be expected to be more pronounced for that year.

Table 4. Summary of Pedestrian-Motor Vehicle Crashes by Year for the Florida DHSMV Database and the Zone Database.

\begin{tabular}{|l|c|c|r|r|r|c|}
\hline \multirow{2}{*}{\begin{tabular}{c} 
Year \\
\cline { 2 - 7 }
\end{tabular}} & $\begin{array}{c}|c| \\
\text { Injury and } \\
\text { No Injury }\end{array}$ & Fatal & Total & $\begin{array}{c}\text { Miami-Dade Zone (Geocoded) Database } \\
\text { Injury and } \\
\text { No Injury }\end{array}$ & Fatal & Total \\
\hline 1996 & 1,865 & 97 & 1,962 & 1,698 & 91 & 1,789 \\
\hline 1997 & 1,859 & 82 & 1,941 & 1,686 & 67 & 1,753 \\
\hline 1998 & 1,889 & 86 & 1,975 & 1,537 & 67 & 1,604 \\
\hline 1999 & 1,833 & 82 & 1,915 & 1,558 & 78 & 1,636 \\
\hline 2000 & 1,914 & 82 & 1,996 & 1,704 & 80 & 1,784 \\
\hline 2001 & 2,016 & 71 & 2,087 & 1,850 & 71 & 1,921 \\
\hline 2002 & 1,860 & 86 & 1,946 & 1,698 & 85 & 1,783 \\
\hline 2003 & 1,690 & 75 & 1,765 & 1,535 & 69 & 1,604 \\
\hline 2004 & 1,658 & 63 & 1,721 & 1,536 & 62 & 1,598 \\
\hline Total & 16,584 & 724 & 17,308 & 14,802 & 670 & 15,472 \\
\hline
\end{tabular}

\section{Analysis Groups and Data Subsets}

The data analysis plan for the evaluation involved several separate examinations in order to assess whether the demonstration program successfully reduced crashes. These were:

- The trend of annual crashes in Miami-Dade over time from the period before the program's interventions through the period during which the program was actively attempting to reduce pedestrian crashes;

- A comparison of the rate of change in pedestrian crashes in Miami-Dade over time with the crash experience in the rest of Florida outside of Miami-Dade;

- A comparison of the rate of change in pedestrian crashes in Miami-Dade over time with the crash experience in similar and contiguous counties; and

- Analysis of crash trends in the four high-crash zones discussed earlier that were defined based on crash occurrence before the start of the program intervention. 
Addressing each of these examinations required forming several subsets of crash data from the overall file maintained by DHSMV. Various characteristics of the DHSMV file required different manipulations of the total database to extract subsets as follows:

\section{Generating Pedestrian Crash Maps}

The matched addresses were used to create an electronic map of the pedestrian crashes, as illustrated in Figure 5 for the before-analysis period of 1996-2000. The maps were made in early 2001 for use in countermeasure selection, so data were not yet available for 2001 at that time. Each red dot represents one pedestrian crash that occurred. The crash location (pin) map can be analyzed to reveal different crash-related factors, such as age of pedestrian, injury severity, light conditions (Figure 6), and crash type, based on data from the police report. The pedestrian crash data was combined with other Miami-Dade County GIS data to show the relationship of crashes to other spatial data, such as locations of schools (Figure 7), nursing homes (Figure 8), transit stops, and aerial images. These and other pedestrian crash maps were used at the zone level in the process of countermeasure development, as discussed later in more detail. 


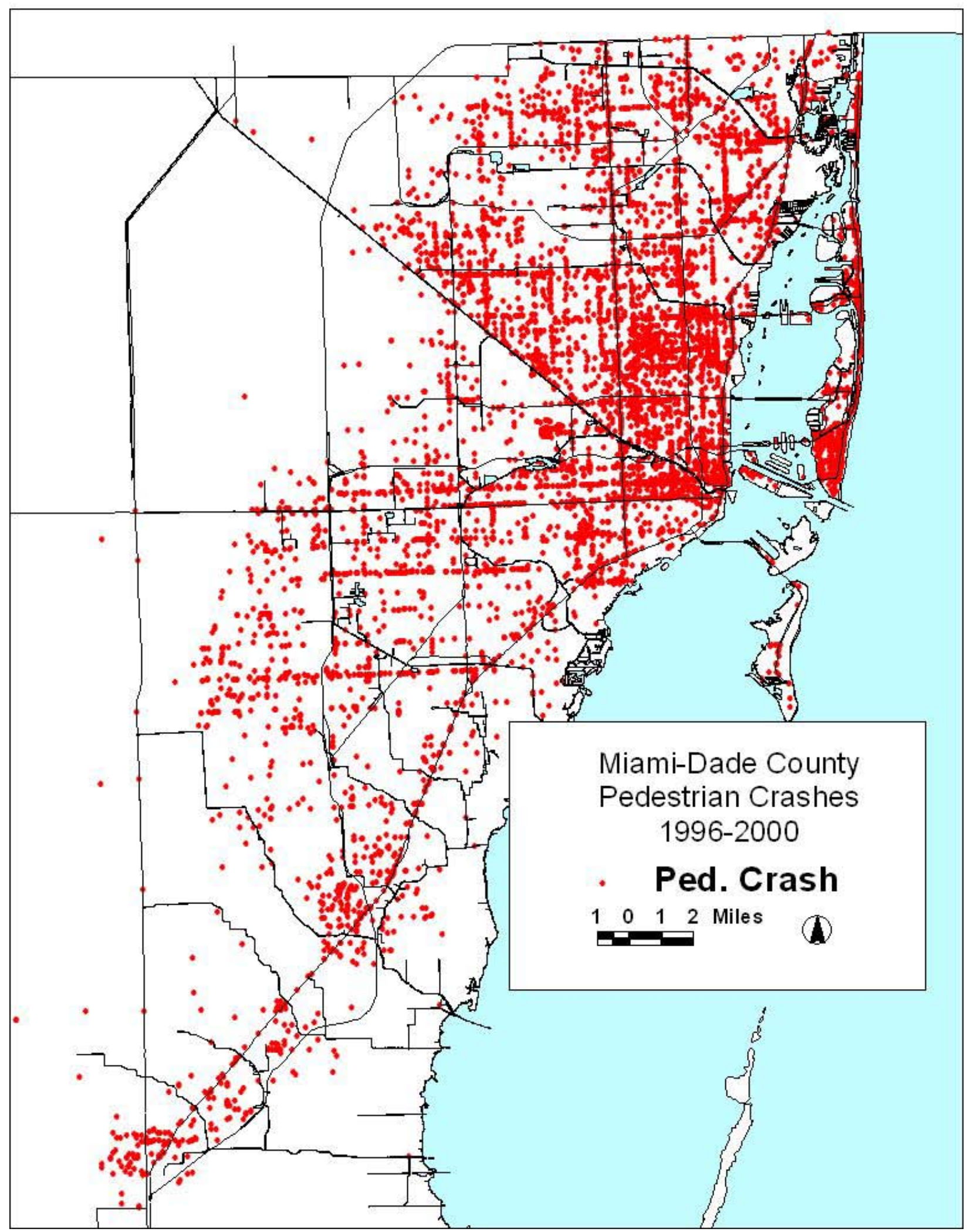

Figure 5. Map of All Miami-Dade County Pedestrian Crashes, 1996 to 2000. 


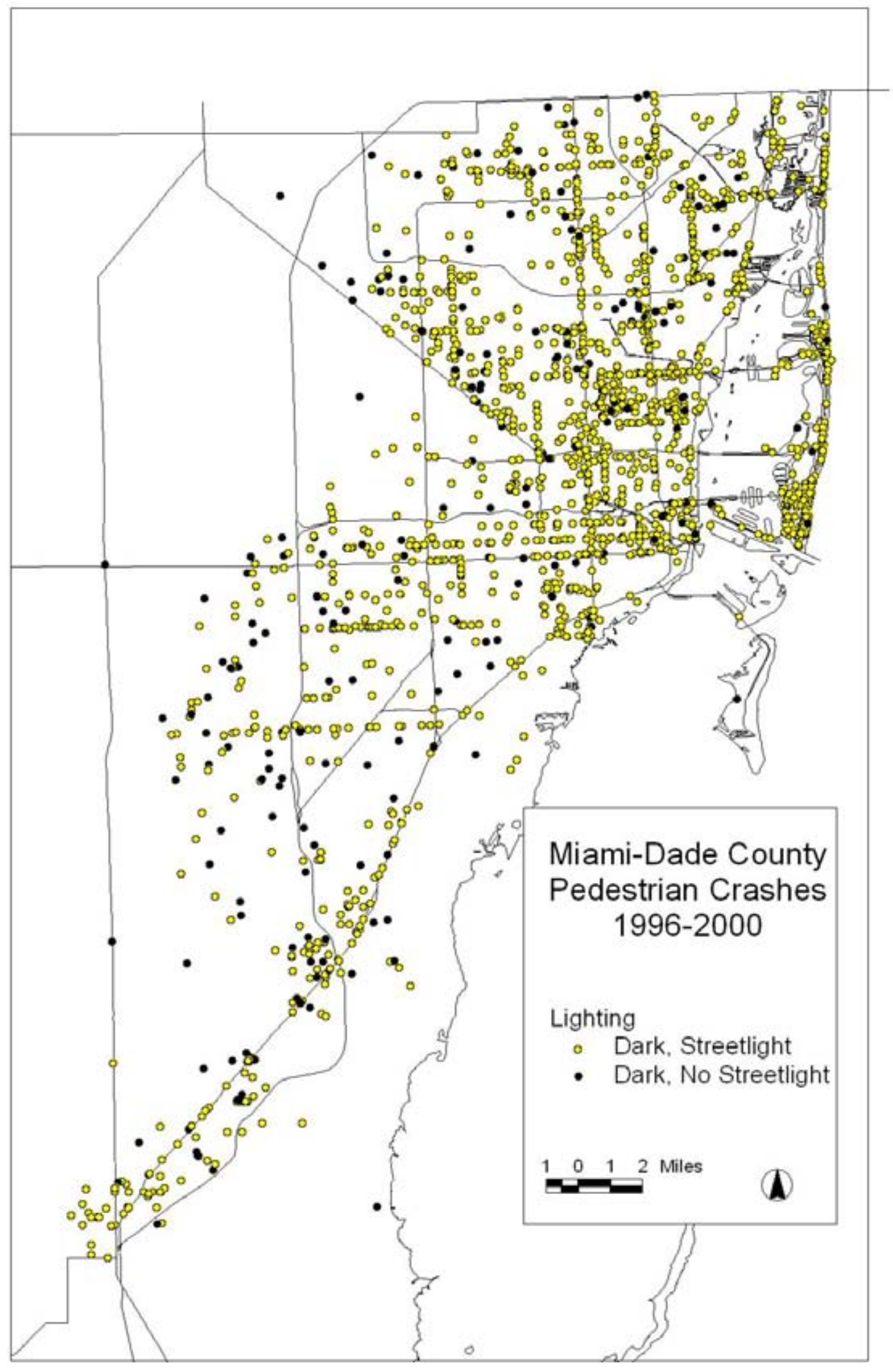

Figure 6. Pedestrian Crashes by Lighting Conditions. 


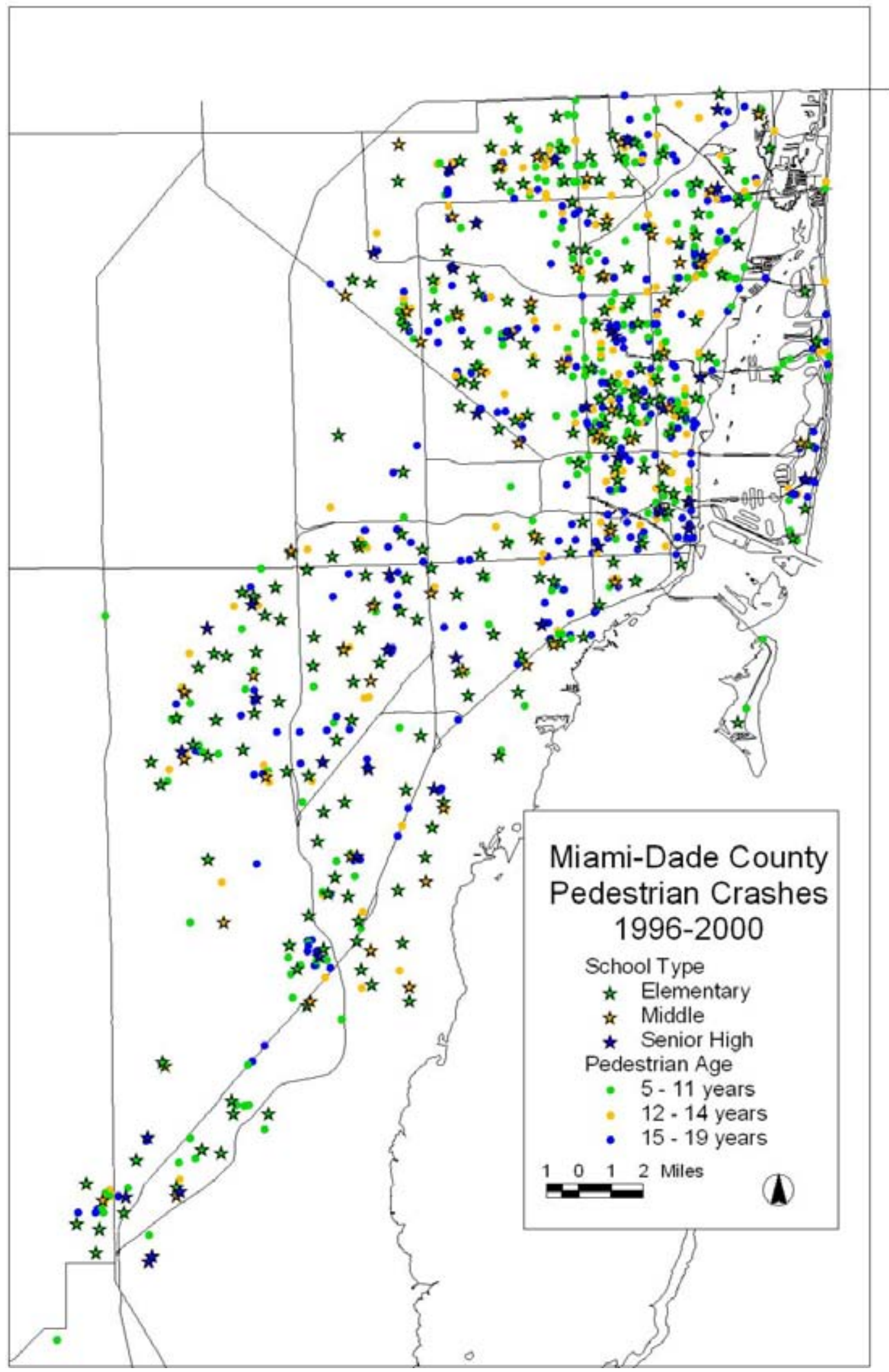

Figure 7. Map of Pedestrian Crashes in Relation to Schools. 


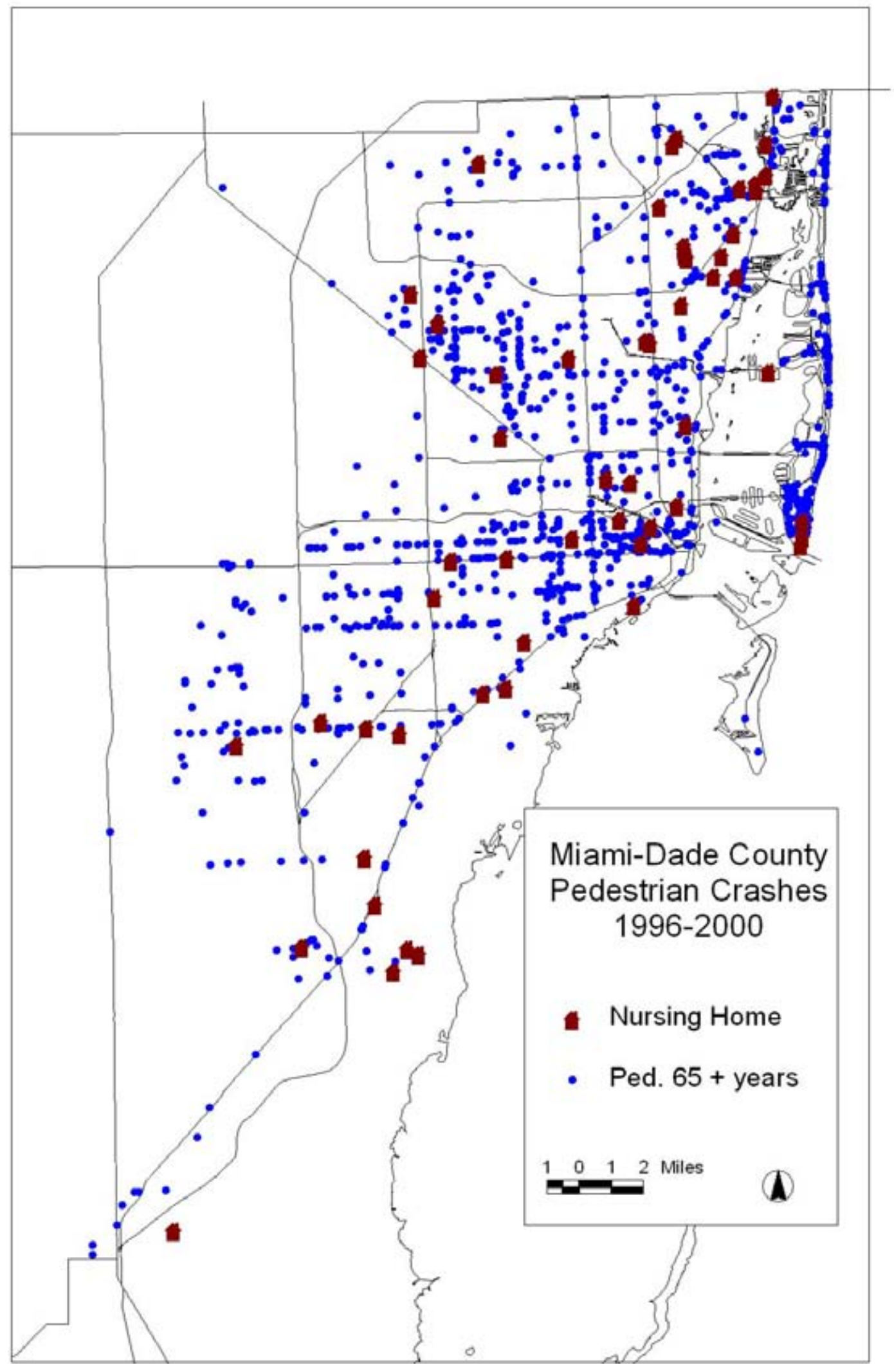

Figure 8. Map of Pedestrian Crashes in Relation to Nursing Homes. 
The GIS system contains an algorithm that calculates the density of data points based on search criteria provided by the user. The crash density per acre is shown in bands of color that reveal areas where greater numbers of pedestrian crashes have occurred. This feature was used to identify high-crash areas, corridors, and intersections for prioritizing countermeasure resources. The end result of this process is an electronic map of the pedestrian crashes, where high-crash concentration areas can be displayed by the ArcView software (see Figure 9). 


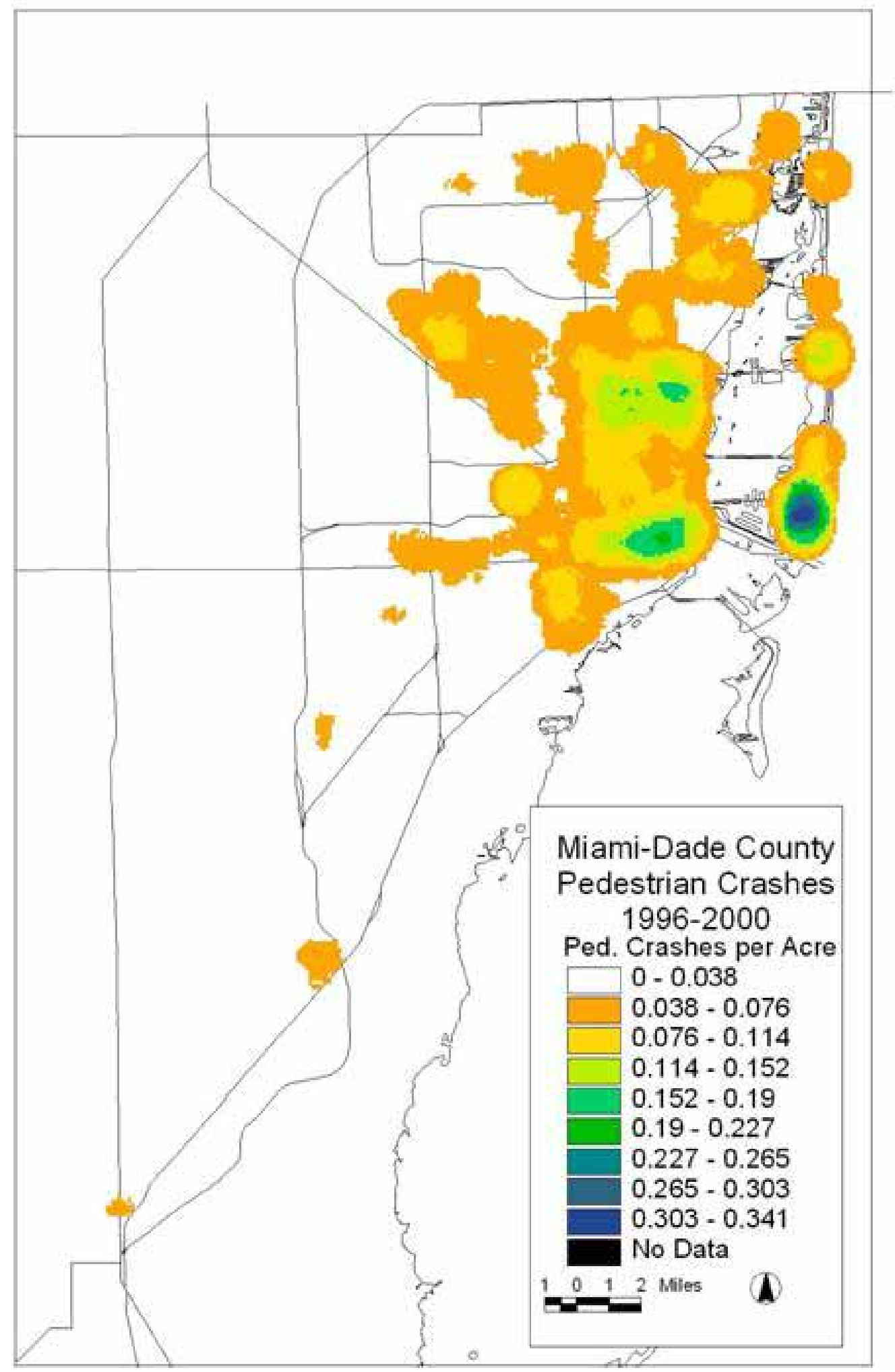

Figure 9. Miami-Dade Pedestrian Crash Concentration Areas. 
Based on this analysis, four zones were identified for further detailed investigation and targeted pedestrian safety measures. These zones included South Beach, Liberty City, Little Havana, and Little Haiti, as shown in Figure 10. The area size of the identified four zones is 9,891 acres, less than $1 \%$ of the total area size of Miami-Dade County. However, during 1996 to 2004, the number of pedestrian crashes in the four zones comprised up to $20 \%$ of the total number of pedestrian crashes in Miami-Dade County $(3,078$ of 15,474). See Table 5 for annual crash frequencies by year and crash zone. Results of the crash problem analyses are reported in Chapter 5.

Table 5. Annual Crash Frequency by Year and Crash Zones.

\begin{tabular}{|c|c|c|c|c|c|c|c|c|c|c|}
\hline Zones & Age group & 1996 & 1997 & 1998 & 1999 & 2000 & 2001 & 2002 & 2003 & 2004 \\
\hline \multirow{5}{*}{$\begin{array}{l}\text { Liberty } \\
\text { City }\end{array}$} & $0-13$ & 38 & 43 & 44 & 34 & 30 & 35 & 37 & 22 & 22 \\
\hline & $14-64$ & 67 & 81 & 64 & 68 & 72 & 70 & 69 & 48 & 63 \\
\hline & $65+$ & 13 & 6 & 8 & 5 & 6 & 12 & 5 & 2 & 8 \\
\hline & $5-12$ & 28 & 34 & 35 & 15 & 25 & 21 & 19 & 15 & 15 \\
\hline & Missing & 16 & 7 & 5 & 0 & 4 & 5 & 0 & 2 & 0 \\
\hline \multirow{5}{*}{$\begin{array}{l}\text { Little } \\
\text { Haiti }\end{array}$} & $0-13$ & 26 & 13 & 12 & 10 & 21 & 14 & 19 & 8 & 14 \\
\hline & $14-64$ & 29 & 32 & 32 & 34 & 32 & 36 & 43 & 31 & 36 \\
\hline & $65+$ & 5 & 3 & 7 & 4 & 5 & 5 & 8 & 2 & 3 \\
\hline & $5-12$ & 23 & 8 & 9 & 4 & 16 & 10 & 8 & 6 & 9 \\
\hline & Missing & 3 & 5 & 2 & 0 & 4 & 7 & 0 & 8 & 0 \\
\hline \multirow{5}{*}{$\begin{array}{l}\text { Little } \\
\text { Havana }\end{array}$} & $0-13$ & 9 & 6 & 10 & 13 & 11 & 11 & 10 & 4 & 10 \\
\hline & $14-64$ & 56 & 36 & 44 & 27 & 48 & 50 & 45 & 49 & 45 \\
\hline & $65+$ & 20 & 17 & 21 & 17 & 18 & 31 & 20 & 29 & 27 \\
\hline & $5-12$ & 3 & 4 & 8 & 4 & 8 & 8 & 3 & 2 & 6 \\
\hline & Missing & 6 & 6 & 9 & 0 & 6 & 6 & 0 & 6 & 0 \\
\hline \multirow{5}{*}{$\begin{array}{l}\text { South } \\
\text { Beach }\end{array}$} & $0-13$ & 6 & 6 & 1 & 5 & 2 & 6 & 2 & 3 & 4 \\
\hline & $14-64$ & 76 & 75 & 67 & 75 & 82 & 73 & 68 & 53 & 56 \\
\hline & $65+$ & 20 & 19 & 14 & 21 & 17 & 8 & 17 & 16 & 14 \\
\hline & $5-12$ & 5 & 4 & 1 & 1 & 1 & 4 & 1 & 2 & 0 \\
\hline & Missing & 9 & 6 & 4 & 0 & 2 & 2 & 0 & 2 & 0 \\
\hline \multirow[t]{5}{*}{ Other } & $0-13$ & 265 & 232 & 222 & 272 & 198 & 219 & 282 & 168 & 233 \\
\hline & $14-64$ & 855 & 892 & 772 & 835 & 939 & 1,032 & 928 & 865 & 854 \\
\hline & $65+$ & 205 & 208 & 208 & 216 & 213 & 229 & 230 & 226 & 209 \\
\hline & $5-12$ & 188 & 171 & 176 & 136 & 133 & 147 & 132 & 127 & 110 \\
\hline & Missing & 65 & 60 & 58 & 0 & 74 & 70 & 0 & 60 & 0 \\
\hline
\end{tabular}

\section{Assigning Crash Type Information}

Once high-crash zones were identified, hard copies of all pedestrian crashes occurring in the zones were reviewed and used to assign a crash type to each crash. PBCAT software was used to produce a file containing the crash report numbers with the numeric and descriptive crash types generated by coding. Crashes that occurred in the high-crash zones for the 1996 to 2001 baseline period and in the 2002 to 2004 implementation phase were typed. The crash type information was added to data from the State crash files, using crash report number to match. Crash types, along with other factors such as pedestrian age and time-of-day of crash were examined to 
develop targeted countermeasures to address specific crash problems. See Appendix A for more on crash types and PBCAT crash codes.

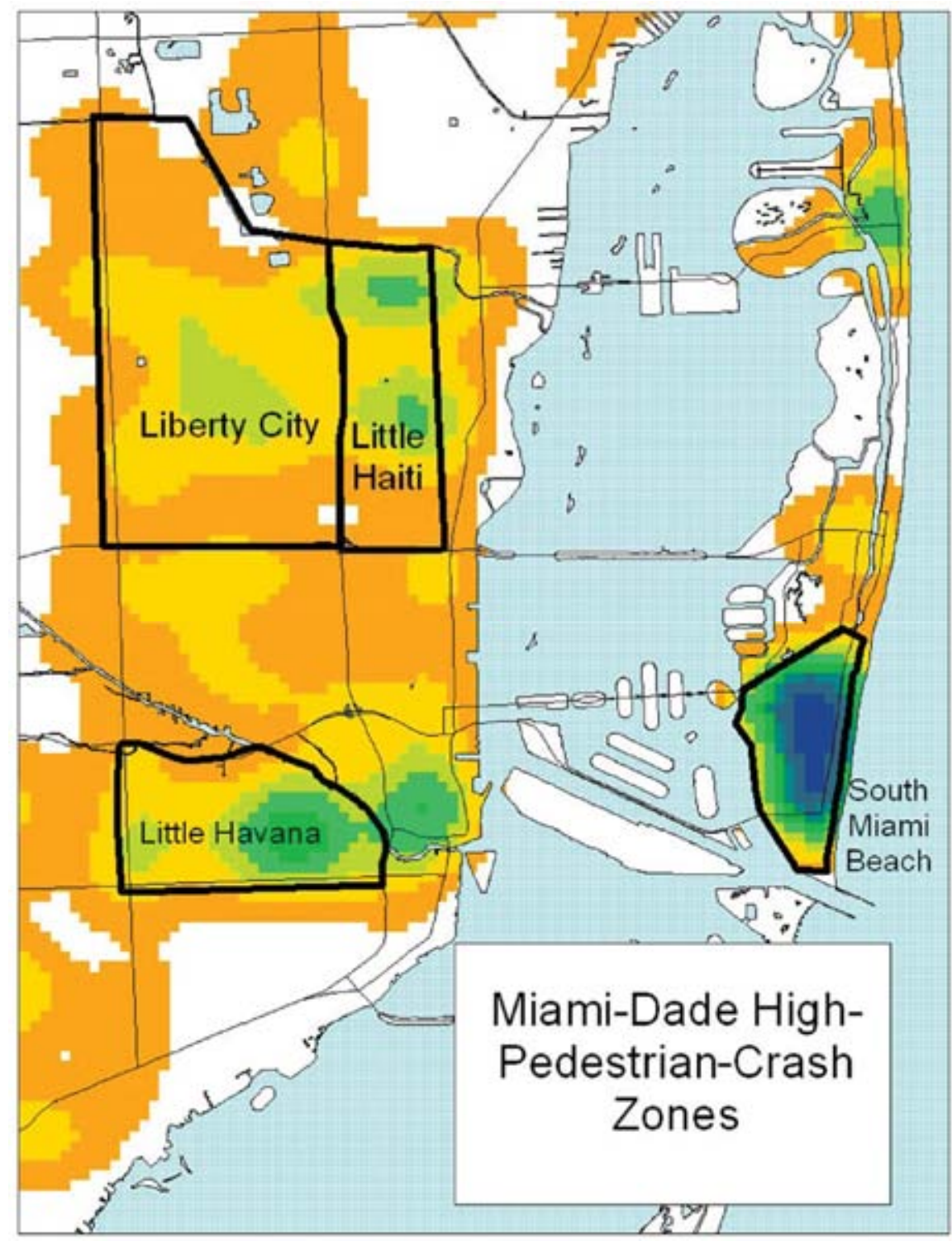

Figure 10. Miami-Dade High-Pedestrian-Crash Zones.

Within each of the four high-crash zones, detailed crash maps were generated to show the pedestrian crash patterns that have occurred along various corridors and at certain intersections. More discussion on each of the zones is provided in the following several sections. Crash data were further analyzed by mapping crashes by pedestrian age, time of day, and other factors. Figure 11 shows the distribution of crashes by age. Figure 12 shows the distribution of crashes by time of day within the different zones. Liberty City and Little Haiti were grouped in these figures as they were adjacent neighborhoods with similar age and crash time distributions. This analysis revealed several general trends that further distinguished each study zone's pedestrian 
problems. For example, crashes in Liberty City and Little Haiti occurred primarily in the morning and afternoon periods and largely involved children and young adults. In Little Havana, a large percentage of the crashes involved older adult pedestrians who were identified on the police reports as Hispanic. In South Beach, a majority of the crashes occurred at night, particularly ones involving young adults, and during weekends.

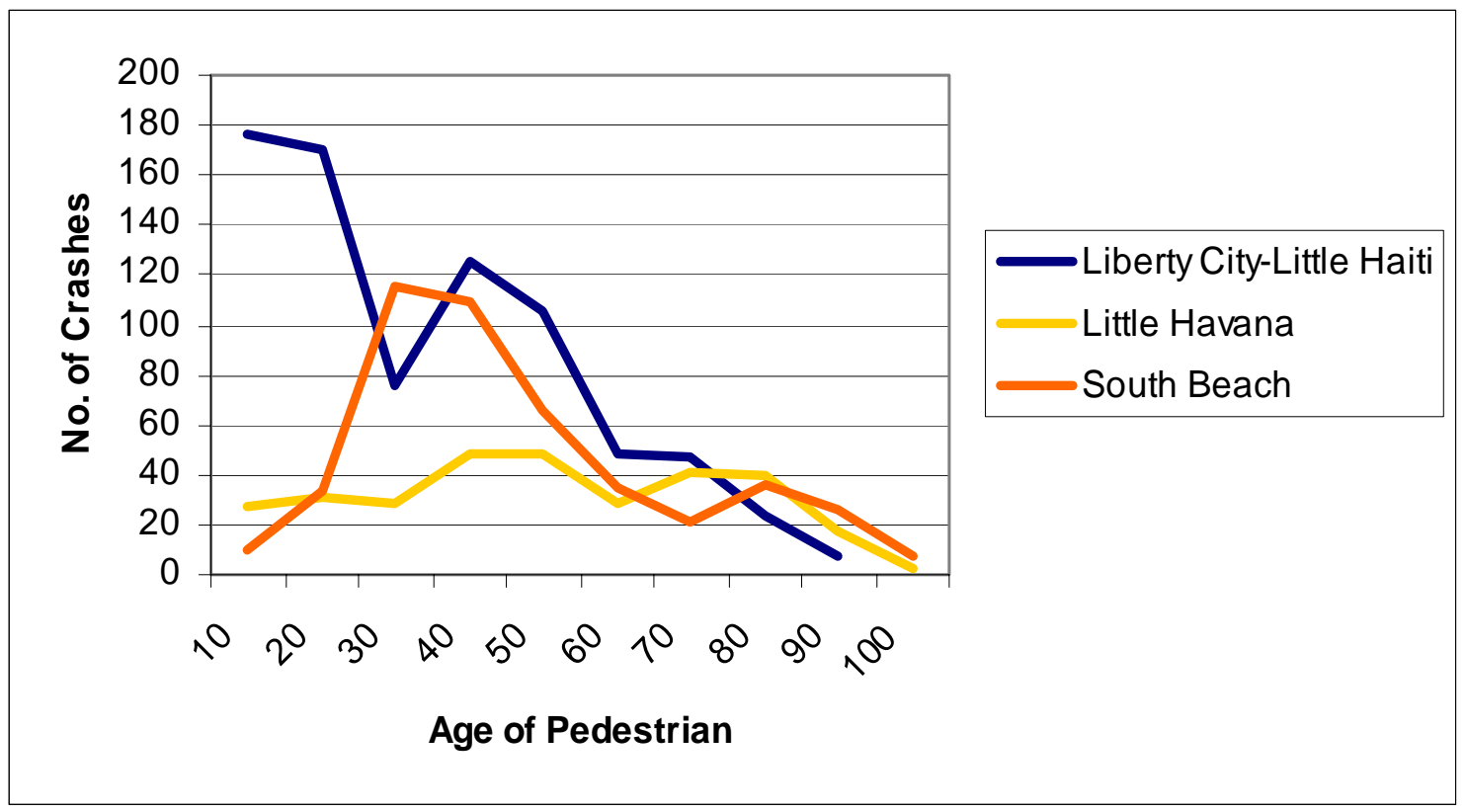

Figure 11. Pedestrian Crashes by Age of Pedestrian.

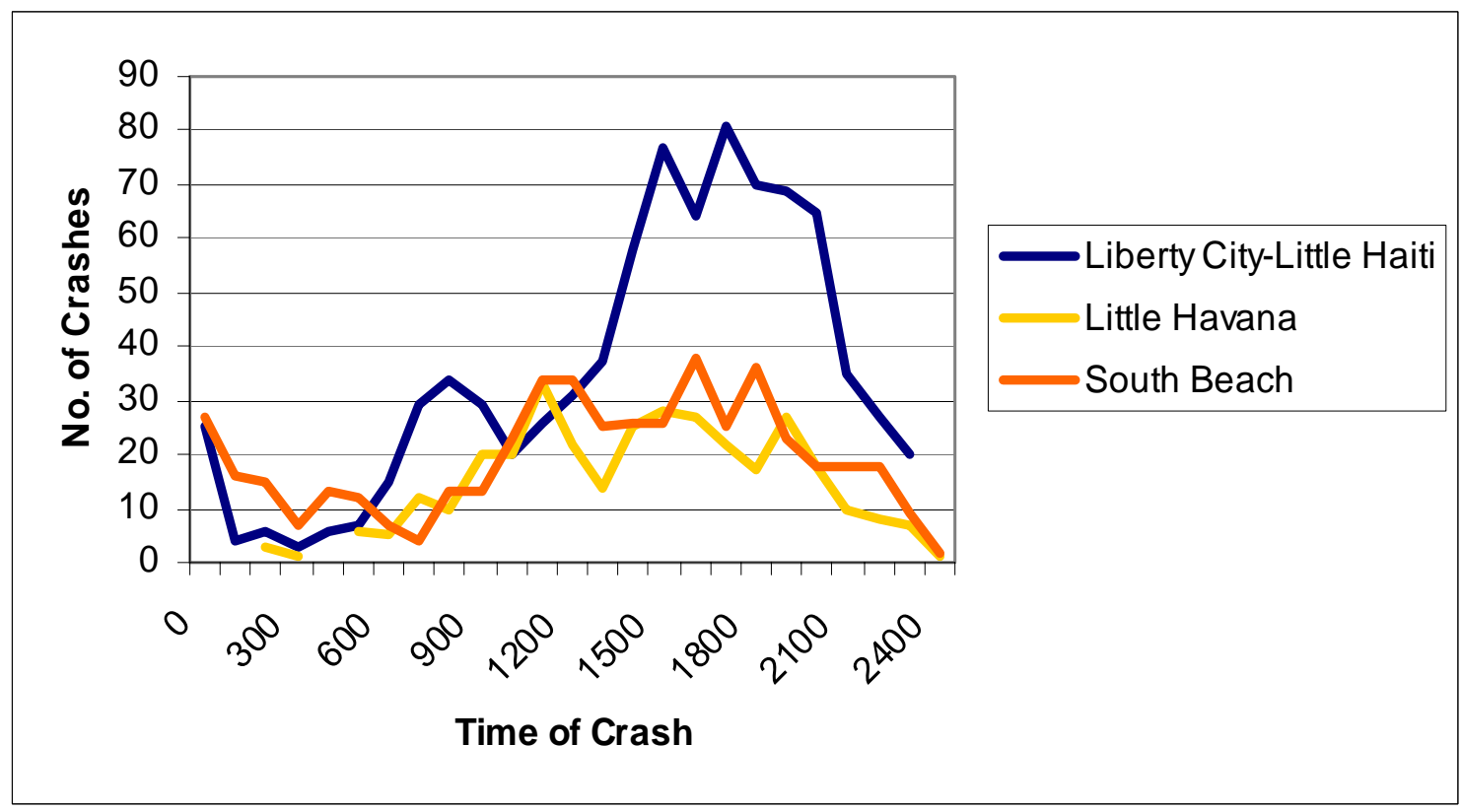

Figure 12. Pedestrian Crashes by Time of Day. 
In each of the four identified high-crash zones, a detailed review was conducted of the crash maps plus individual police crash reports. Project team members also conducted on-site investigations of the high-crash zones and particularly visited each of the high-crash corridors and locations within each of these zones. The investigation team typically included local officials, such as the local and State DOT traffic engineer, as well as a representative of the Miami-Dade MPO (David Henderson) and study team members. Site reviews included:

- Reviewing all of the police crash reports for crashes which occurred at the site or corridor within the past five years;

- Observing site geometrics and traffic control devices, including signs, signals, numbers of lanes, presence and location of on-street parking, location of driveways, etc.;

- Behaviors of motorists and pedestrians, as well as operation of the buses and passengers getting on and off the buses;

- Identifying any obvious or potential problematic roadway features that may have contributed to previous pedestrian crashes; and

- Listing potential engineering, education, and enforcement treatments that may be appropriate at each location.

\section{Description of Study Zones}

A brief description is given below of some of the pedestrian safety and operational issues in the four zones.

\section{$\underline{\text { South Beach }}$}

South Beach is a growing and vibrant city which has changed dramatically in recent years in terms of redevelopment and increased tourism. Many of its streets carry considerable volumes of pedestrians, bicyclists, in-line skaters, and skateboarders, which often interact with motor vehicle traffic. Aerial maps of the overall pedestrian crash problems in South Beach were examined (Figure 13), as well as maps displaying pedestrian crashes by age (Figure 14), severity (Figure 15), crash type, and light condition.

The primary crash features in this zone involved young adult and older pedestrians and a high nighttime crash problem. Dozens of locations and roadway corridors were reviewed by the study team. Based on the on-site investigations and review of crash reports, a variety of specific types of safety problems were found in the South Beach zone which were considered to have contributed to pedestrian crashes. These included:

- Motorists failing to yield to pedestrians in crosswalks and at unsignalized intersections;

- Motorists failing to yield to pedestrians when making right or left turns on green;

- Motorists running red signals, particularly those who make left turns on red (after the opposing through traffic has cleared) and also right turns on red (without stopping and/or without yielding to pedestrians);

- Motorists double-parking, which creates risk to pedestrians and also can contribute to lane-change vehicle crashes; 
- Motorists parking too close to intersections (which blocks sight distance for pedestrians and oncoming motorists);

- Left-turning vehicles failing to yield to pedestrians at intersections and driveways;

- Pedestrian/vehicle conflicts and collisions in parking lots (e.g., shopping center lots);

- Pedestrians walking or running into the street at midblock in front of on-coming traffic; and

- Pedestrians failing to comply with the WALK/DON'T WALK signals.

Some of the roadway and environmental problems that were observed within the zone included:

- Lack of adequate lighting along major routes and at the intersections, particularly in areas with heavy nighttime pedestrian activity;

- The need for traffic and pedestrian signal maintenance and re-timing;

- Pedestrian-related signs in need of replacement;

- Lack of separate left-turn phasing at certain signalized intersections, which contributed to left-turn vehicles striking pedestrians in the crosswalk;

- The need to provide raised medians and/or traffic and pedestrian signals at several sites that had multiple lanes (four or more lanes) combined with traffic volumes of more than 15,000 vehicles per day, where pedestrians were having difficulty crossing safely; and

- Limited or blocked sight distance at intersections and along routes (due to shrubbery next to driveways).

Several of these types of problems are illustrated (Figure 16 through Figure 19). These safety concerns informed decisions that were made about what countermeasures to implement in this zone (a discussion of selected countermeasures is given in Chapter 4). 


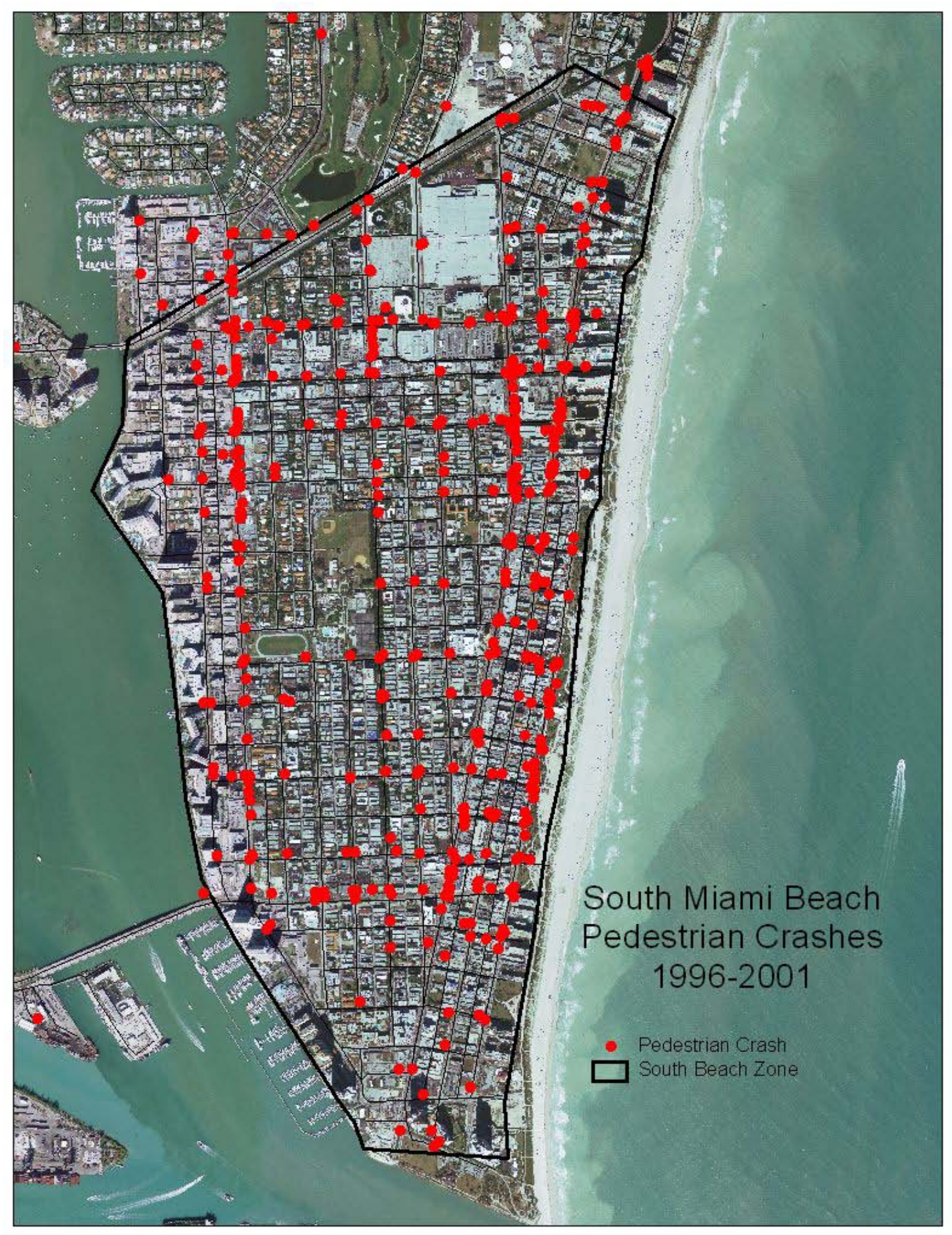

Figure 13. South Beach Aerial Crash Map. 


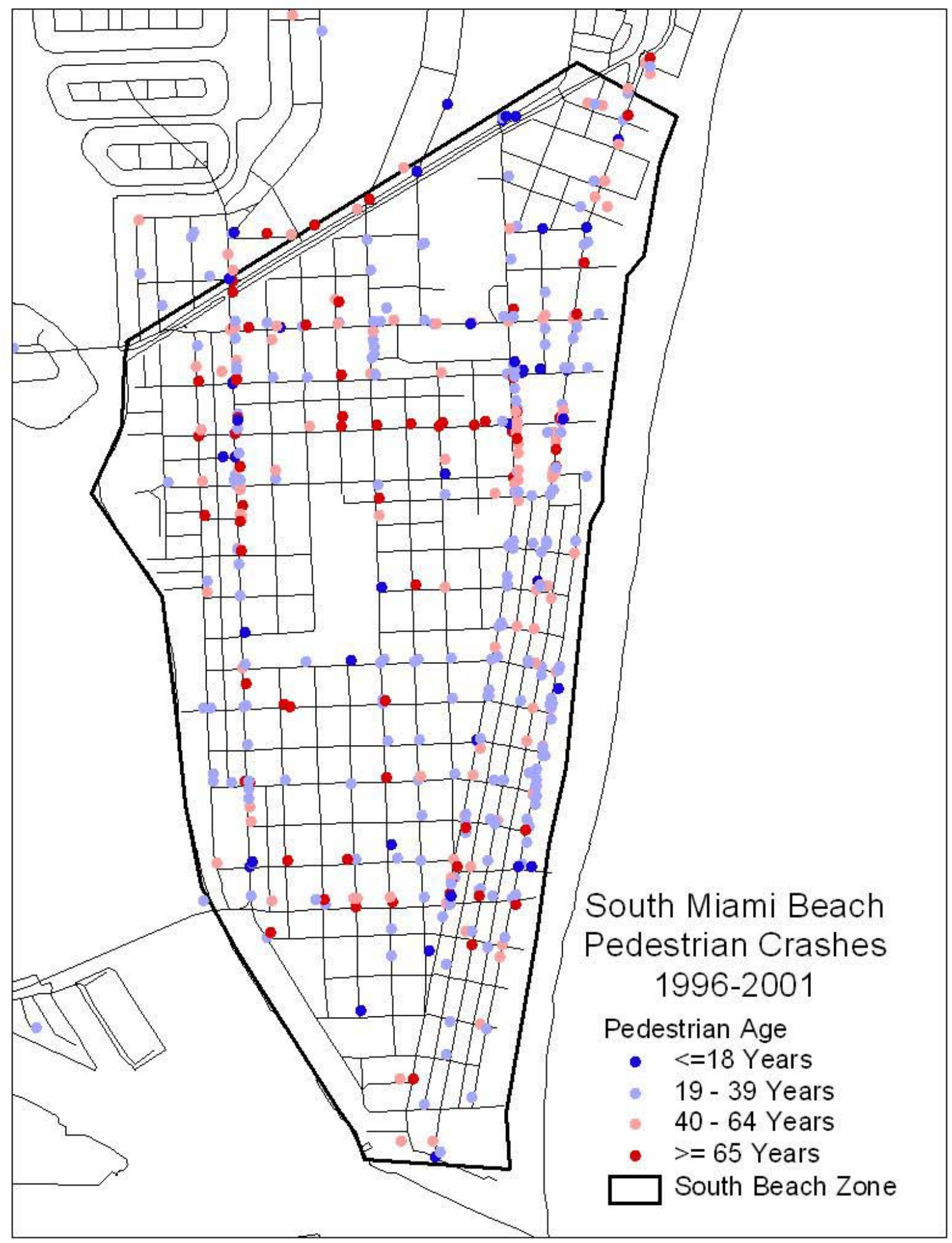

Figure 14. South Beach Crashes by Pedestrian Age. 


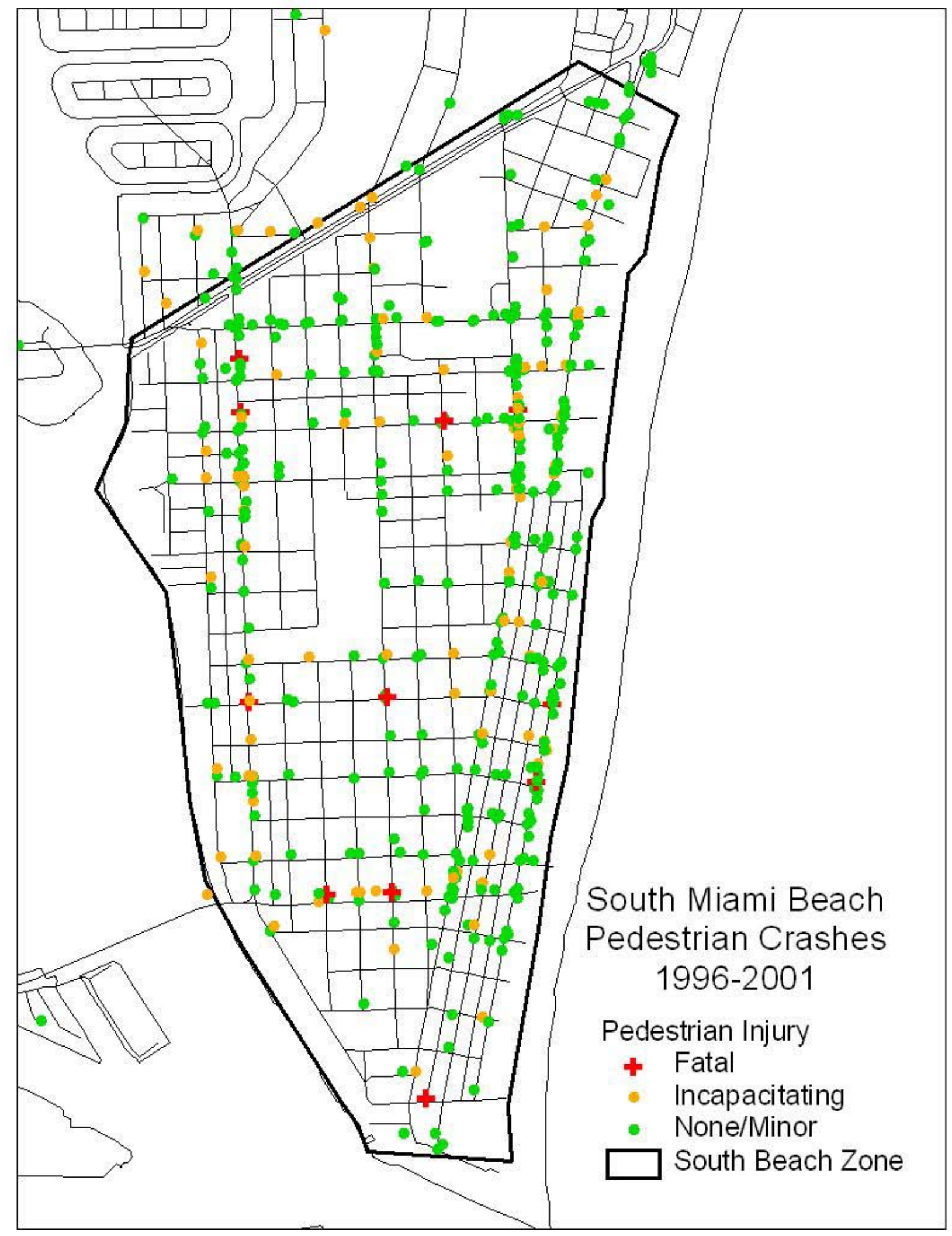

Figure 15. South Beach Crashes by Pedestrian Injury Severity. 


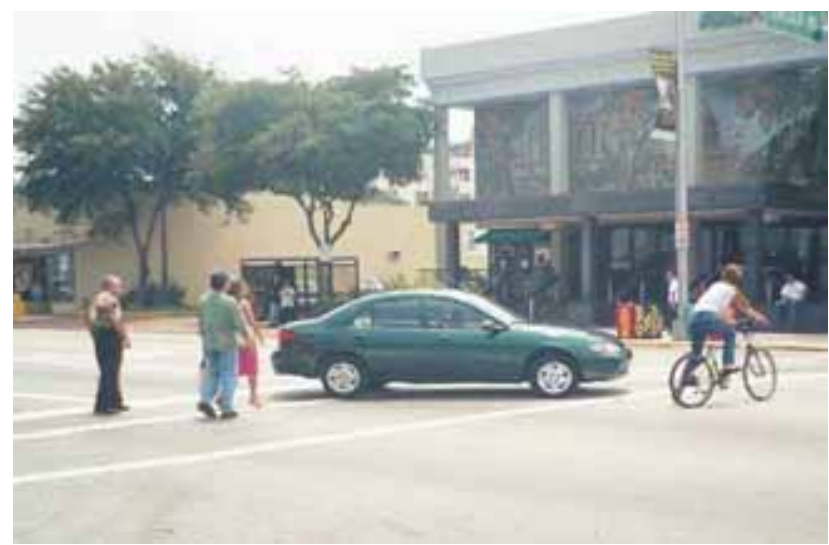

Figure 16. Left-Turning Vehicles Failing to Yield to Pedestrians.

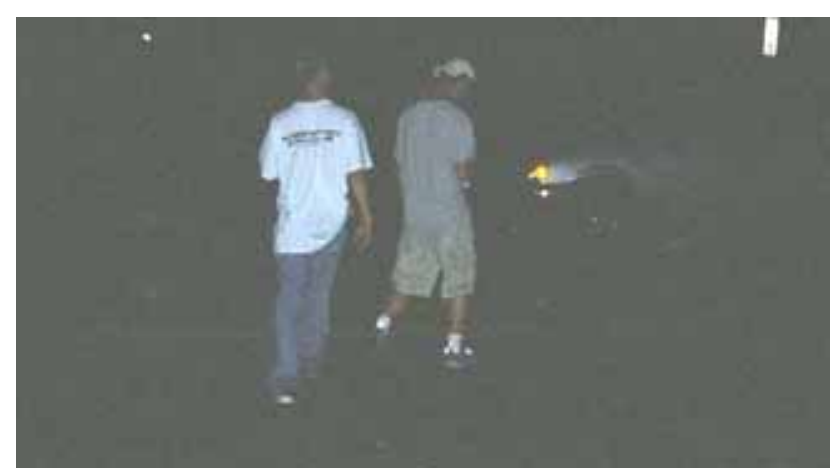

Figure 18. Pedestrian Visibility Issues at Night.

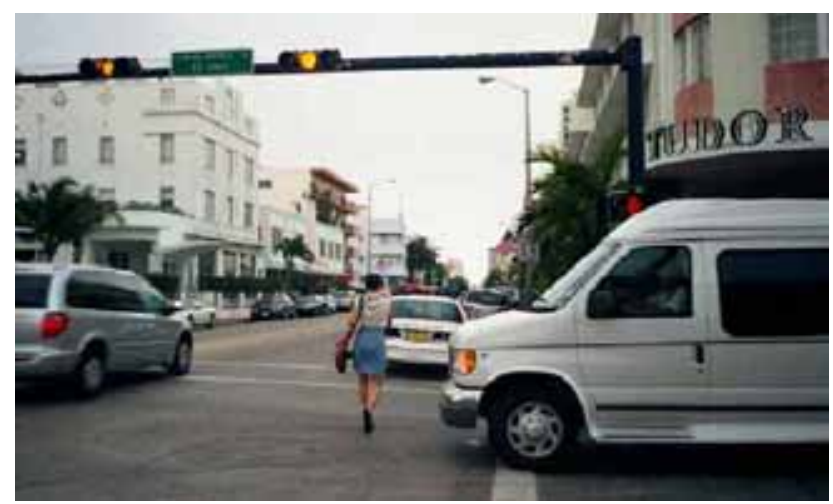

Figure 20. Motorist Failing to Yield to a Pedestrian.

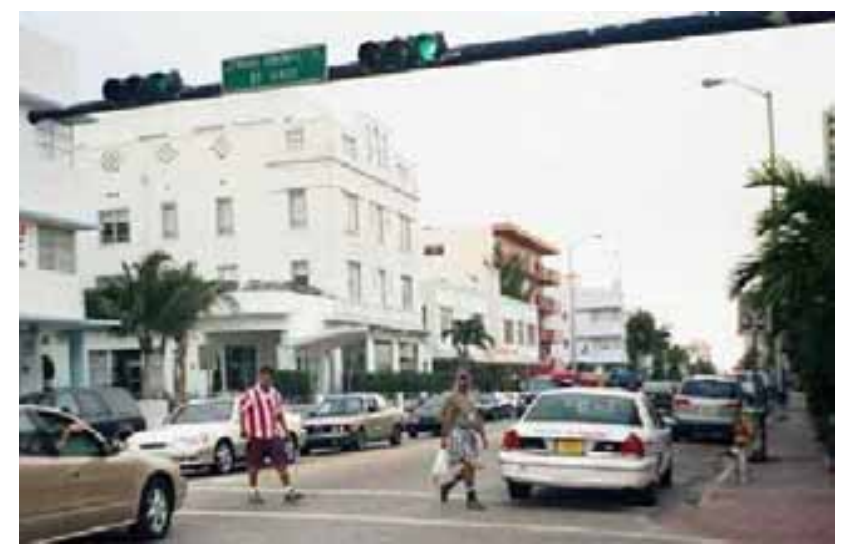

Figure 17. Pedestrians Failing to Obey Traffic Signals.

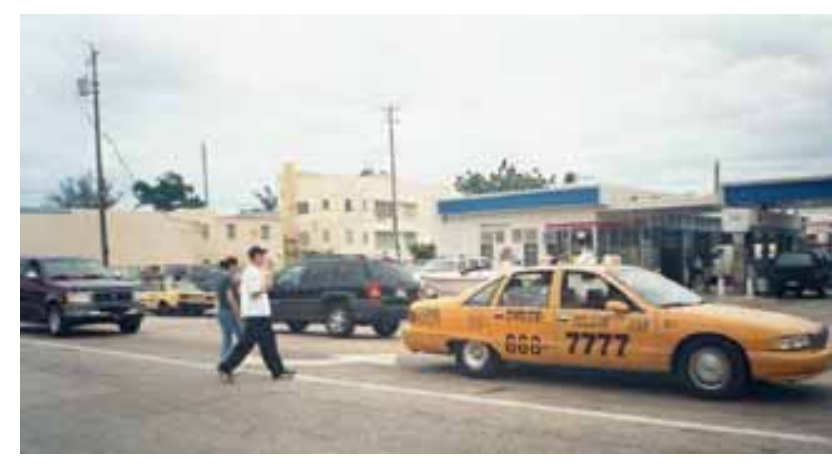

Figure 19. Pedestrians Crossing at Midblock.

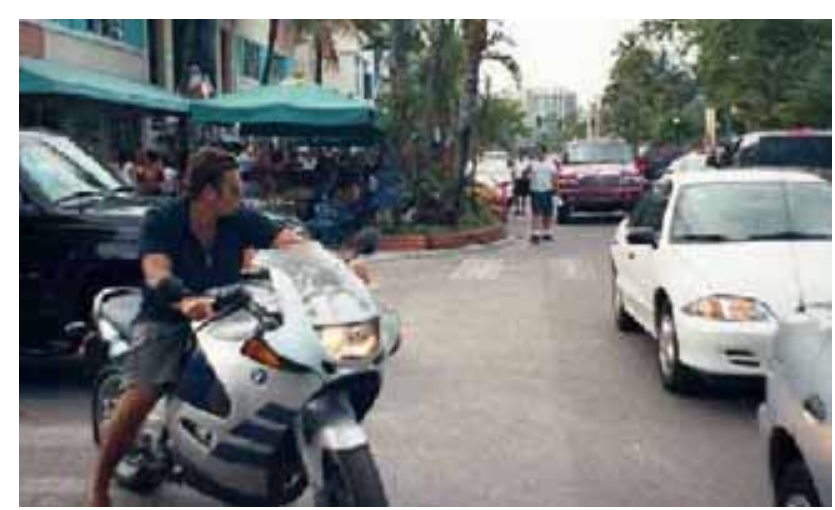

Figure 21. Plantings on Bulbouts Blocking Sight Distance. 


\section{Liberty City and Little Haiti}

These two zones are located in the northwest portion of Miami-Dade County, and they are adjacent to one another. Pedestrian crash maps for Liberty City and Little Haiti are shown in Figure 22 through Figure 24.

The principal crash problems for these two zones involved young children who were struck by motor vehicles while walking to or from school. Liberty City is a predominately low-income community and has a high crime rate. Numerous children were observed being escorted to school by a parent or older family member. Little Haiti, as the name implies, is a community of primarily people of Haitian descent, and many of the inhabitants speak Haitian Creole as their primary language. The crash problems corresponded closely with that in Liberty City; that is, a large portion of the pedestrian crashes involved school children being struck during times when they would be likely be walking to and from school. However, a certain percentage of the crashes involved adults and older adults, particularly trying to cross wide (4- and 5-lane) streets.

Some of the prominent pedestrian safety problems and issues found in these two zones included:

- School children (in both zones) who walk to school at a young age, often unaccompanied by an adult, and attempt to cross wide, busy roads, often at midblock locations without waiting for adequate gaps in traffic before crossing;

- Adult pedestrians (particularly in Little Haiti) who cross 4- and 5-lane arterial streets at midblock by walking to the roadway centerline and standing (sometimes for a minute or more) to wait for traffic to clear before completing their crossing maneuver;

- Motorists who speed on arterial roadways;

- Motorists who do not stop or yield to pedestrians who are trying to cross in a marked or unmarked crosswalk; and

- Pedestrians who cross against the WALK/DON'T WALK signals in the busy downtown intersections.

Some of the roadway and geometric problems included:

- Wide, multilane arterial streets without raised median islands;

- Badly worn signs and crosswalk markings at signalized intersections;

- Poor sight distance at intersections;

- Heavy volumes of pedestrians who cross 4- and 5-lane streets to catch the bus, combined with heavy truck traffic on those routes; and

- School routes in need of traffic engineering enhancements to facilitate safe walking to and from school.

Figure 25 through Figure 28 show examples of some of these situations. Chapter 4 provides a discussion of selected countermeasures for these two zones. 


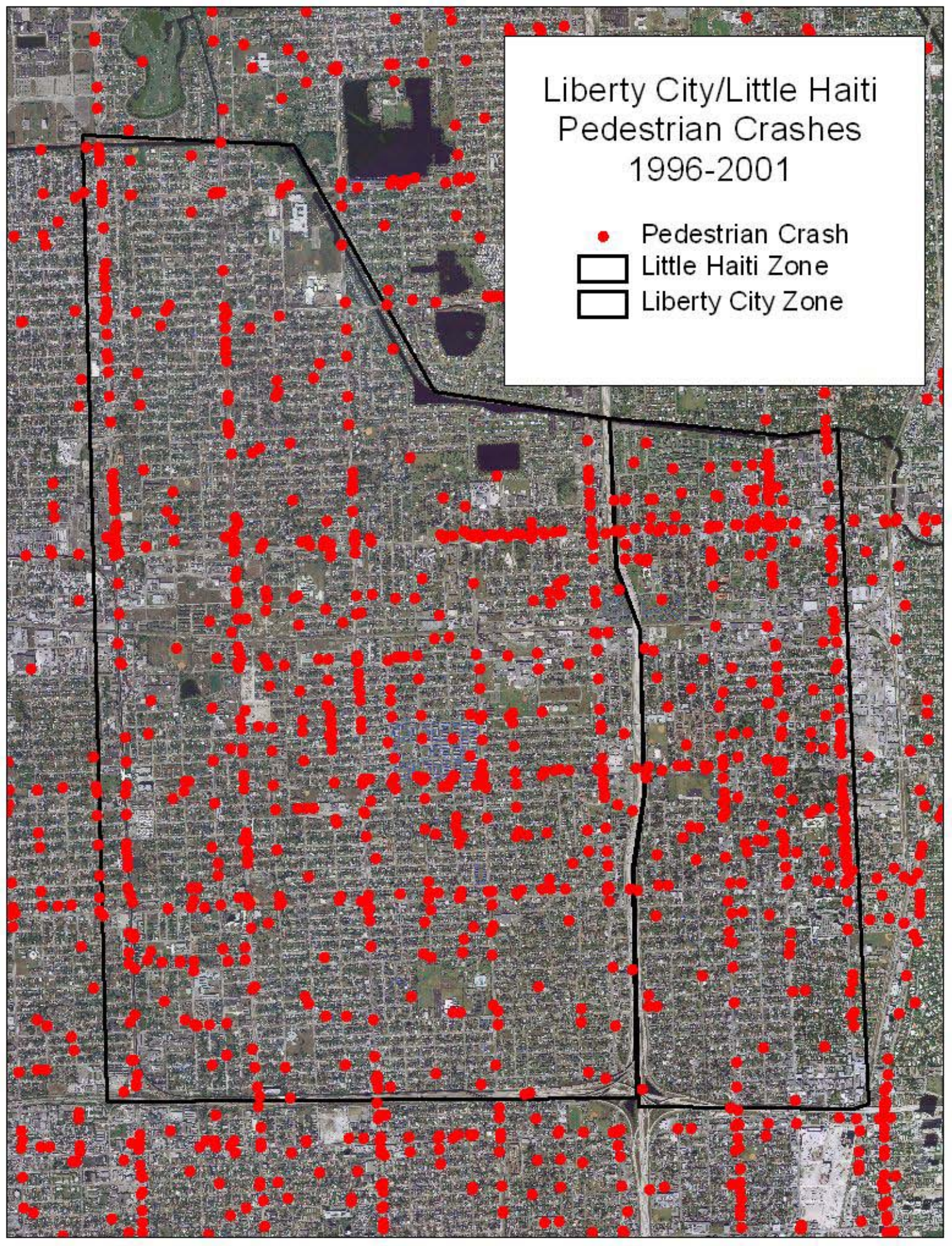

Figure 22. Liberty City/Little Haiti Aerial Crash Map. 


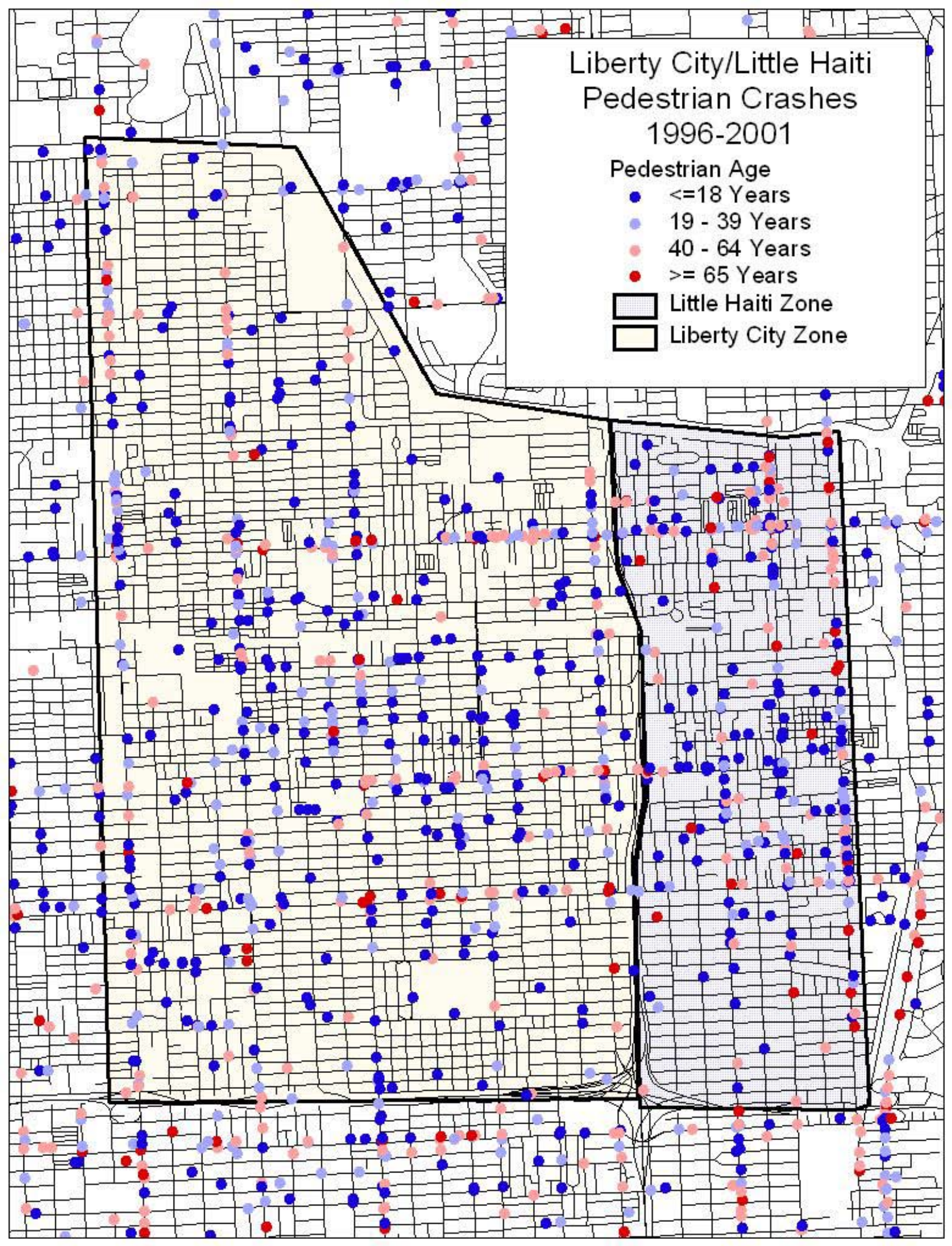

Figure 23. Liberty City/Little Haiti Crashes by Pedestrian Age. 


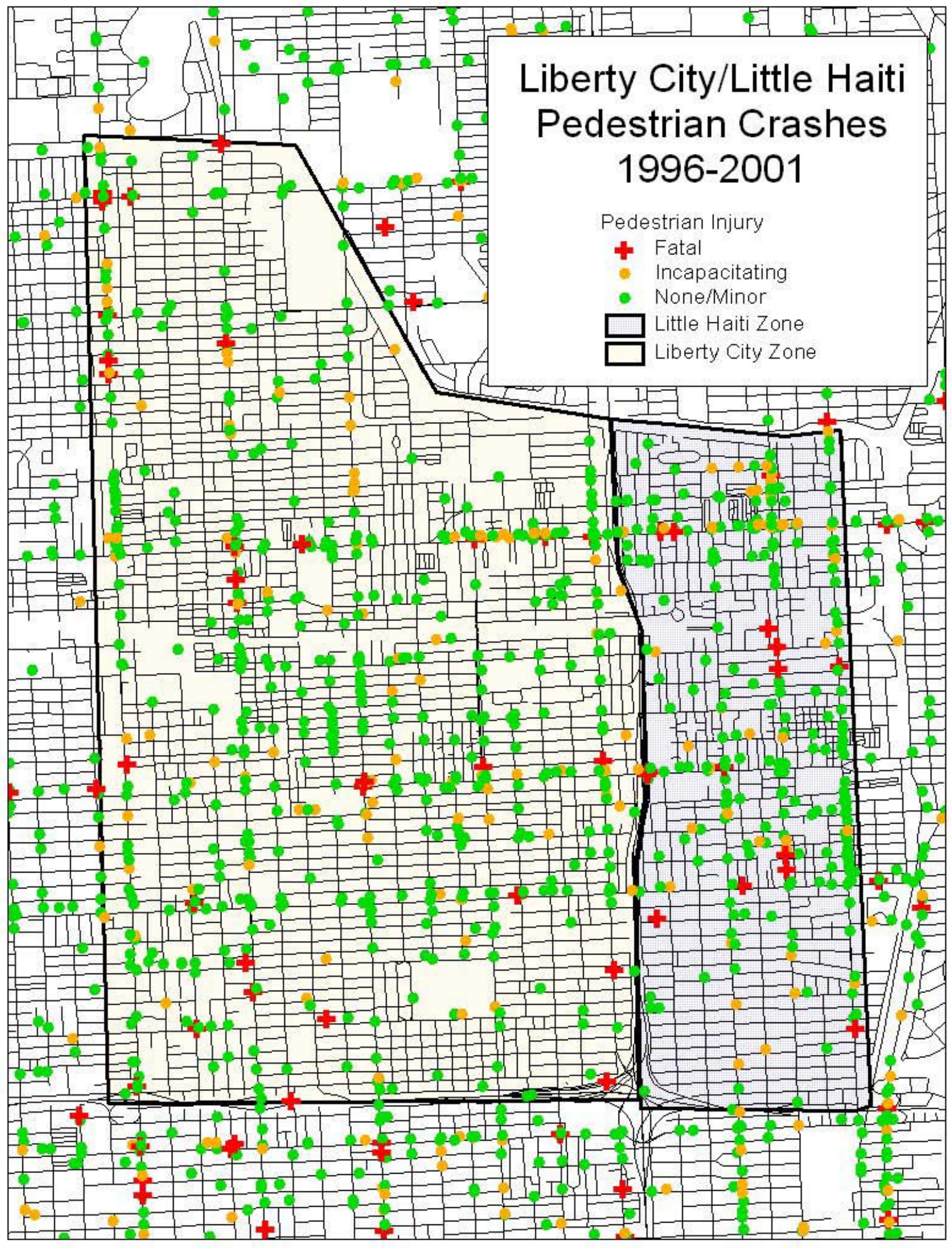

Figure 24. Liberty City/Little Haiti Crashes by Pedestrian Injury Severity. 


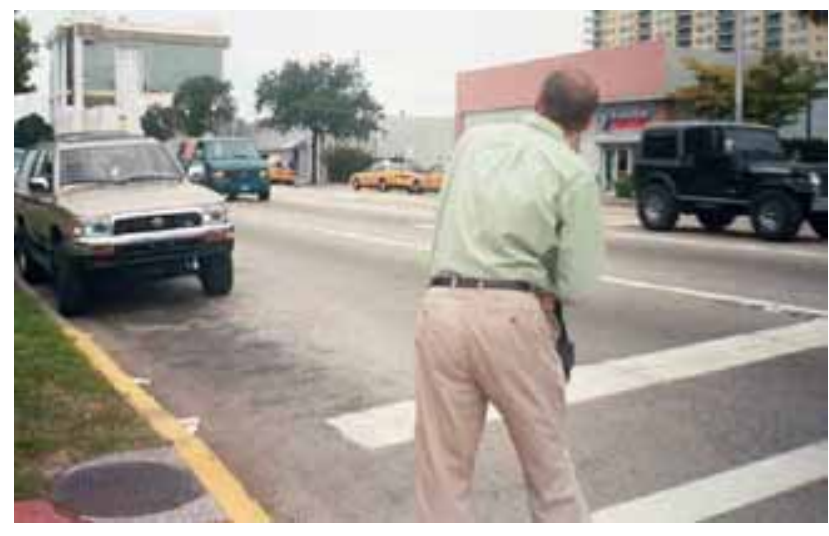

Figure 25. Sight Distance Issues at Intersections.

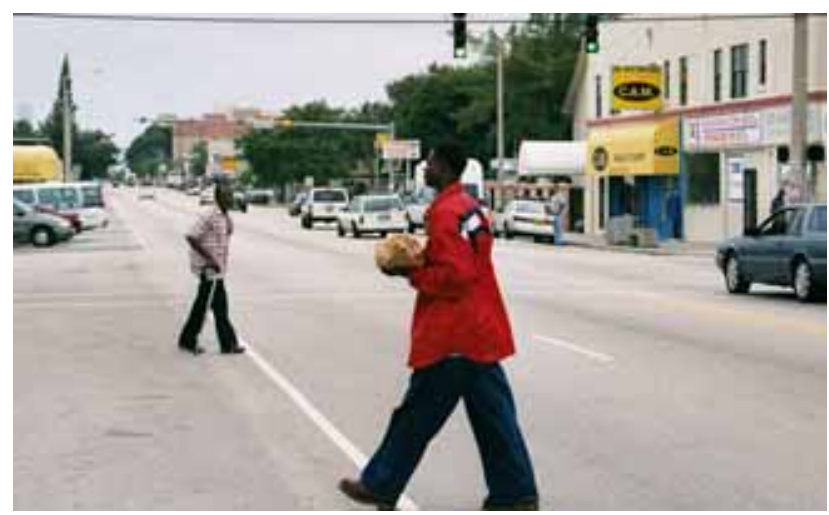

Figure 27. Pedestrian Crossings at Midblock Locations.

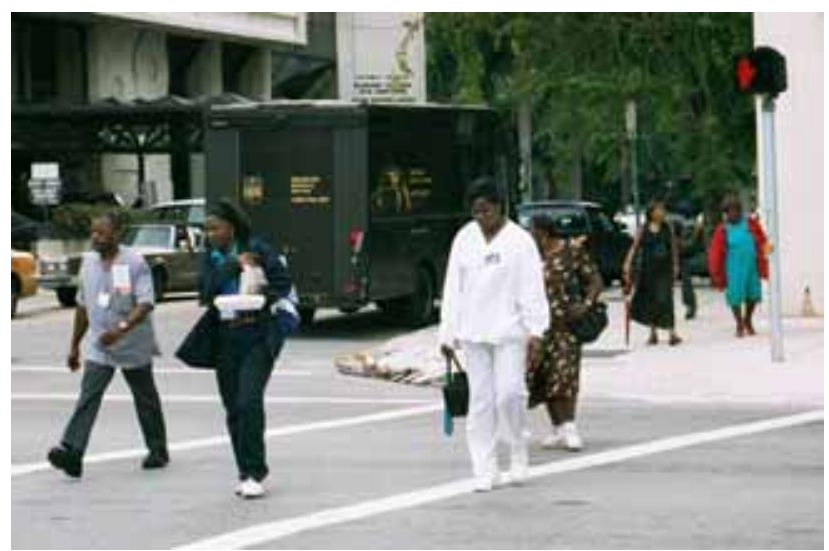

Figure 29. Pedestrian Signal Violations.

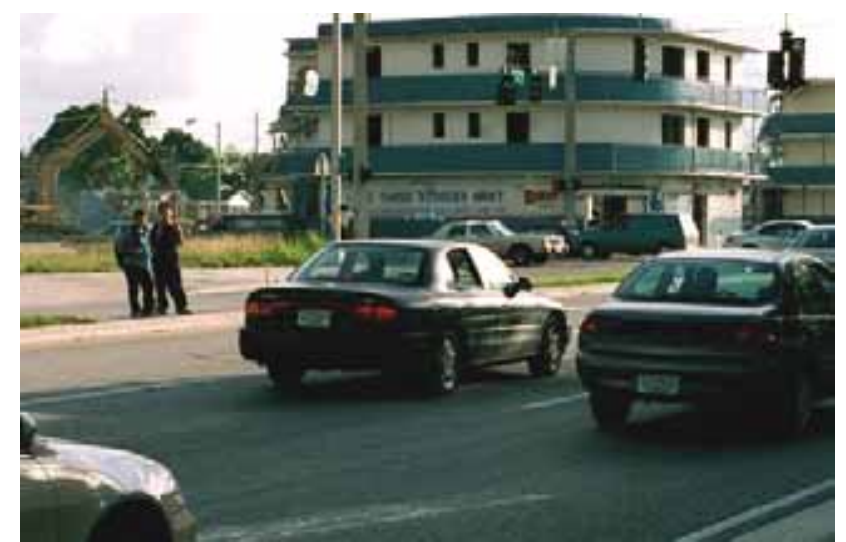

Figure 26. Middle School Children Walking to School.

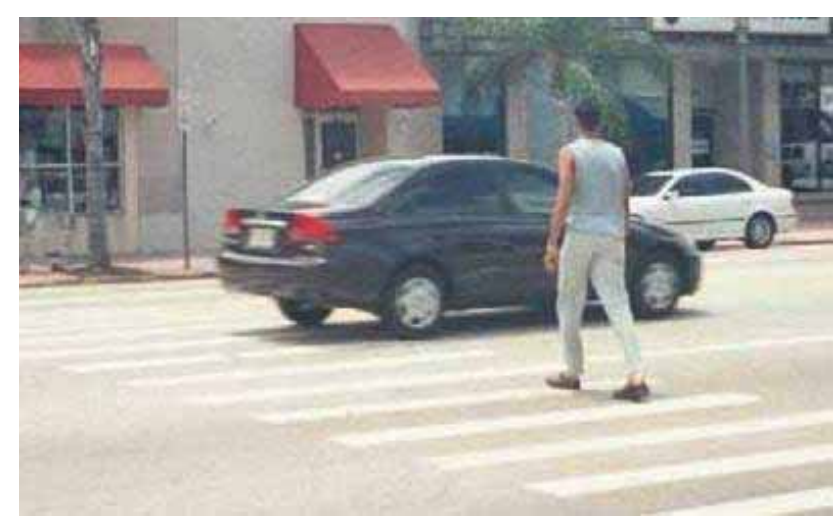

Figure 28. Drivers Fail to Yield to Pedestrians in Crosswalks.

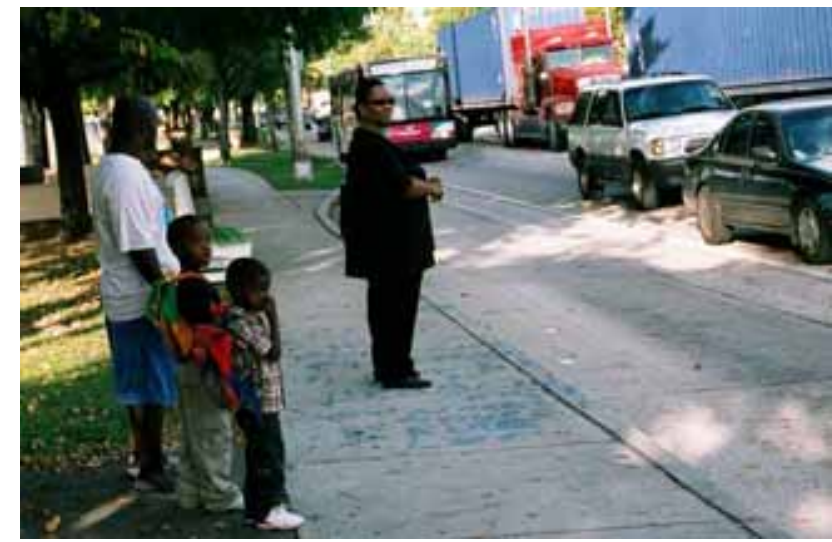

Figure 30. Children Wait for School Bus in Liberty City. 


\section{Little Havana}

Little Havana's population is largely Hispanic, with a substantial percentage of people of Cuban origin. Consequently, Spanish is the predominant language in this zone. A high percentage of the pedestrian crashes involved older pedestrians of Hispanic descent. The crash maps for this zone are shown in Figure 31 through Figure 33. Some of the behaviors observed during the site visits included:

- Pedestrians who cross wide, multilane streets at midblock without adequately searching for oncoming traffic;

- Pedestrians who cross the street at signalized locations but ignore the WALK/DON'T WALK signals;

- Pedestrians who cross against the traffic signal or at midblock between parked cars at some of the high schools and middle schools;

- Motorists who travel at excessive speeds and fail to yield to pedestrians in marked or unmarked crosswalks; and

- Motorists who fail to yield to pedestrians when making right or left turns at signalized intersections, including motorists making right-turns on red without yielding to pedestrians.

Some of the roadway or location features found to contribute to the pedestrian safety problems included:

- Wide, multilane arterial streets with numerous lanes (4- to 6-lane roads) in need of raised median islands;

- Sight-distance problems on intersection corners;

- Buses that stop on the near side of intersections, resulting in some pedestrians getting off the bus and attempting to cross the street in front of the bus, hidden from same-direction traffic;

- The need for Americans With Disabilities Act-compliant curb ramps and pedestrian push-button signals at intersections; and

- Narrow or missing sidewalk links, or sidewalks that are partially blocked by utility and light poles, trees, and other obstructions.

Figure 34 through Figure 37 show examples of some of these situations. Chapter 4 provides a discussion of recommended countermeasures for this zone. 


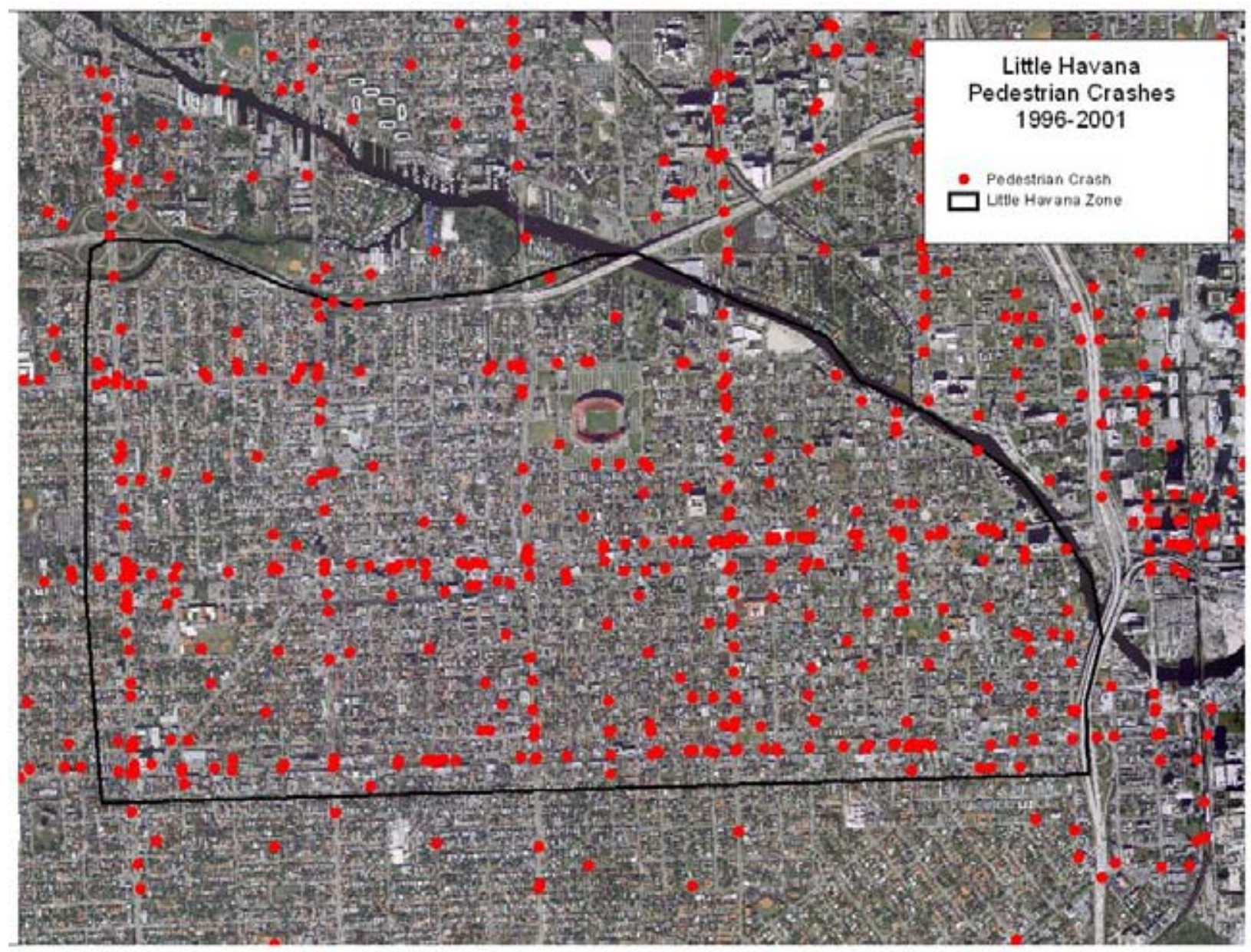

Figure 31. Little Havana Aerial Crash Map. 


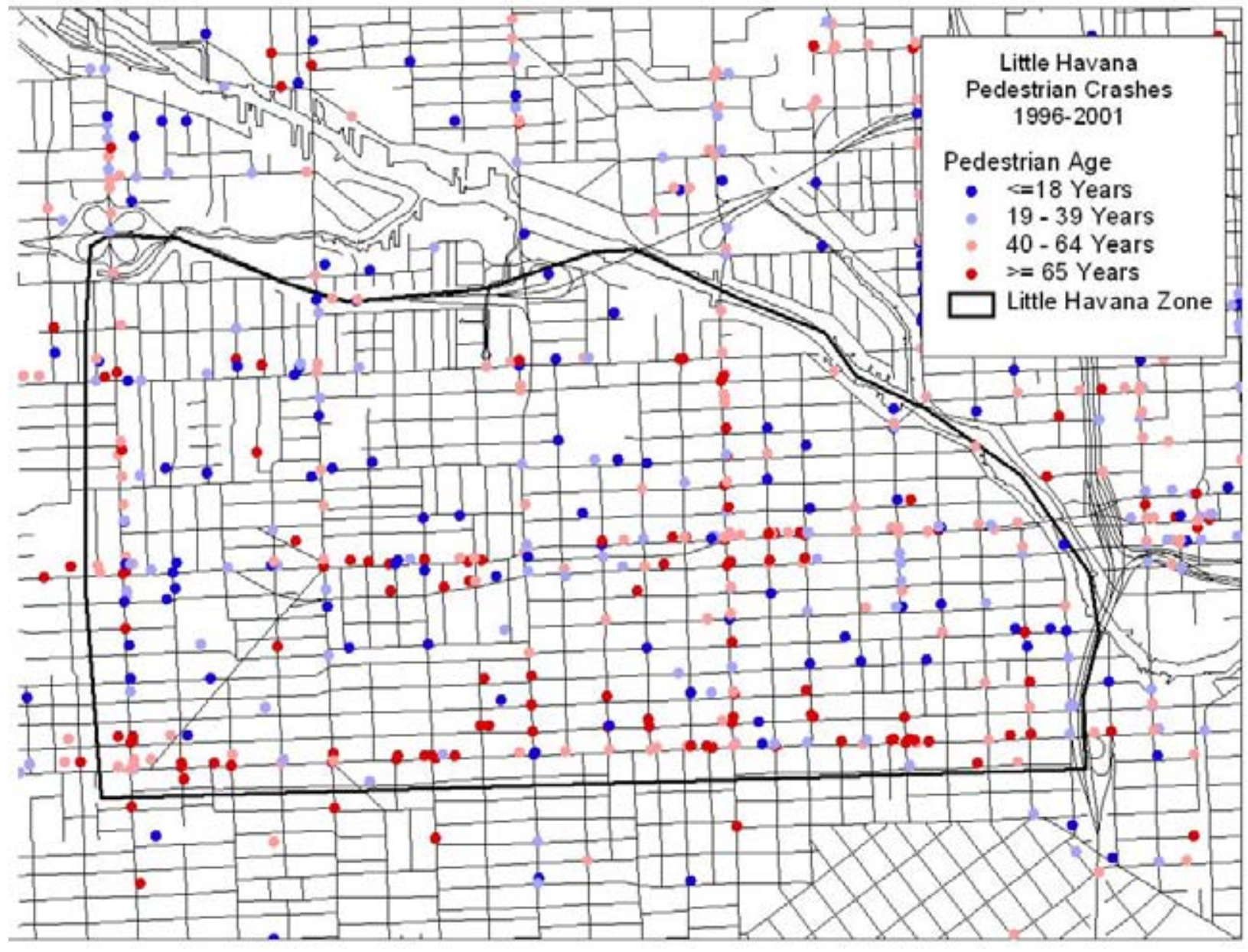

Figure 32. Little Havana Crashes by Pedestrian Age. 


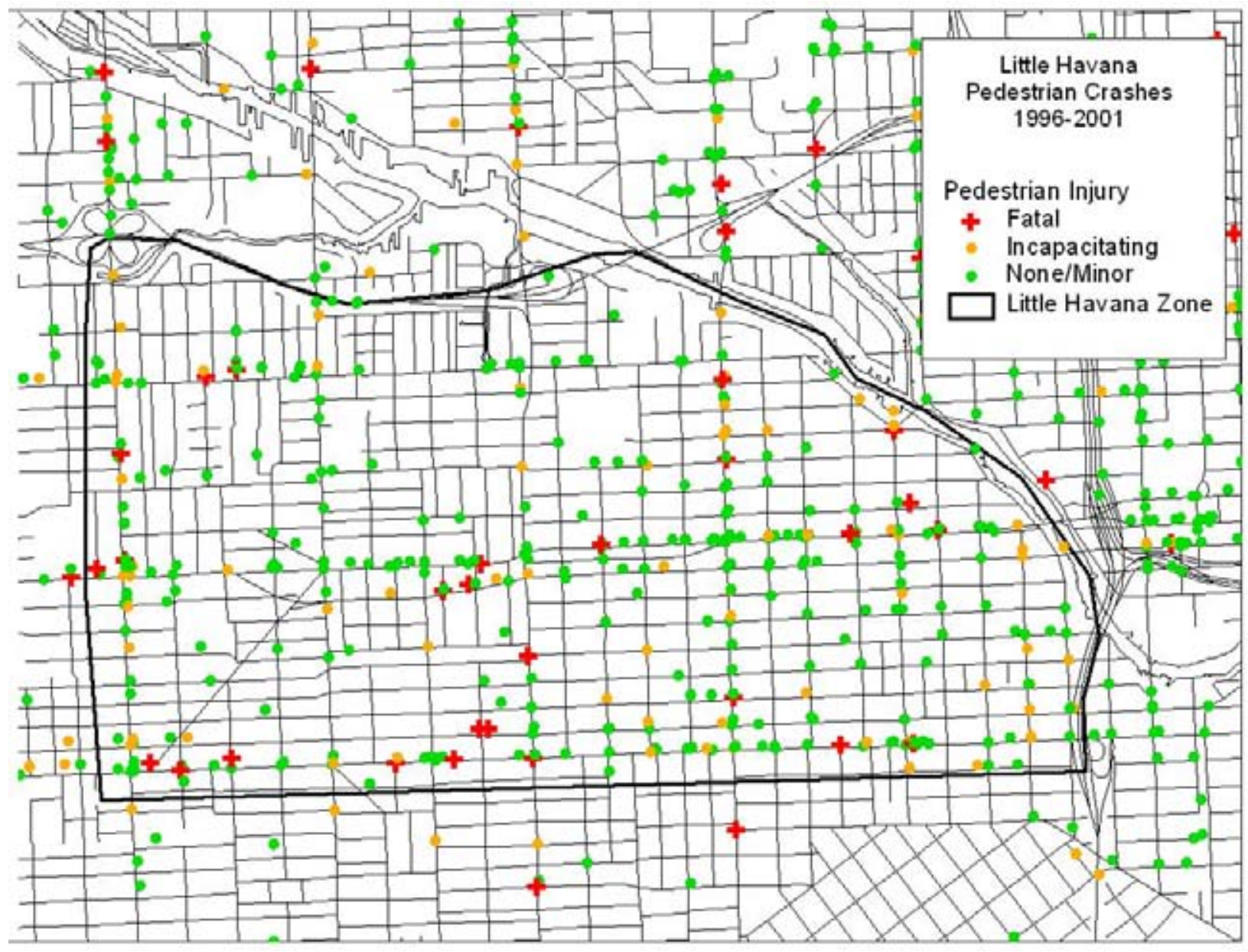

Figure 33. Little Havana Crashes by Pedestrian Injury Severity. 


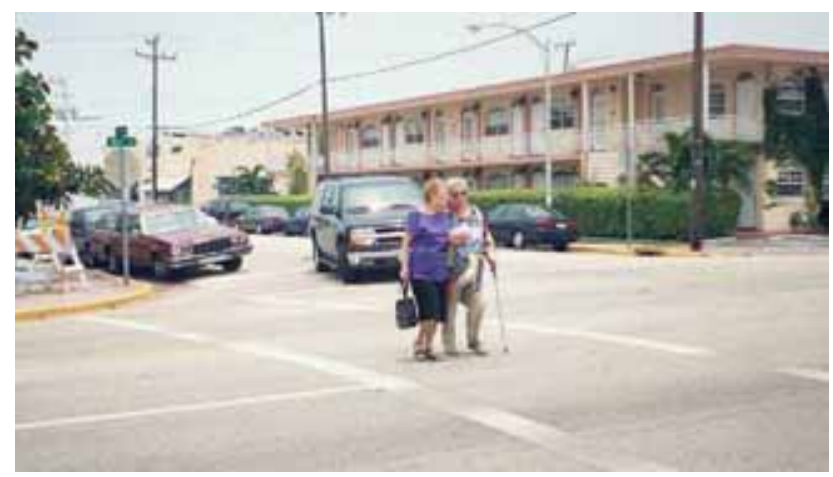

Figure 34. Older Pedestrians Crossing Wide Streets.

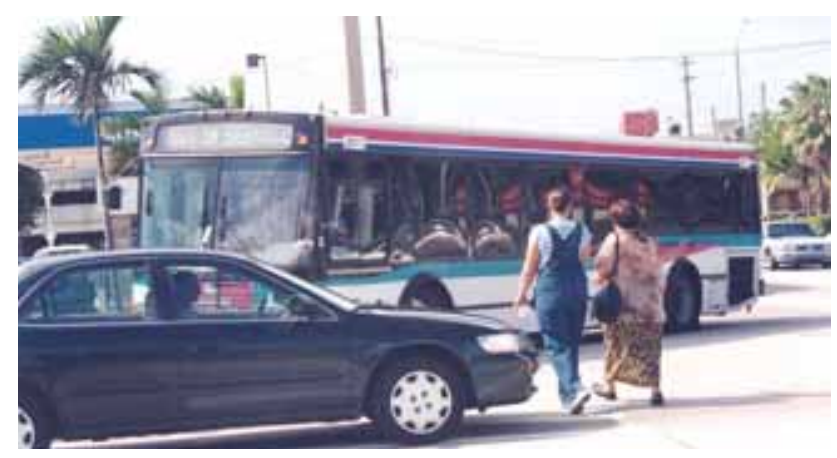

Figure 36. Pedestrians Crossing Street to Catch a Bus in Conflict With Cars.

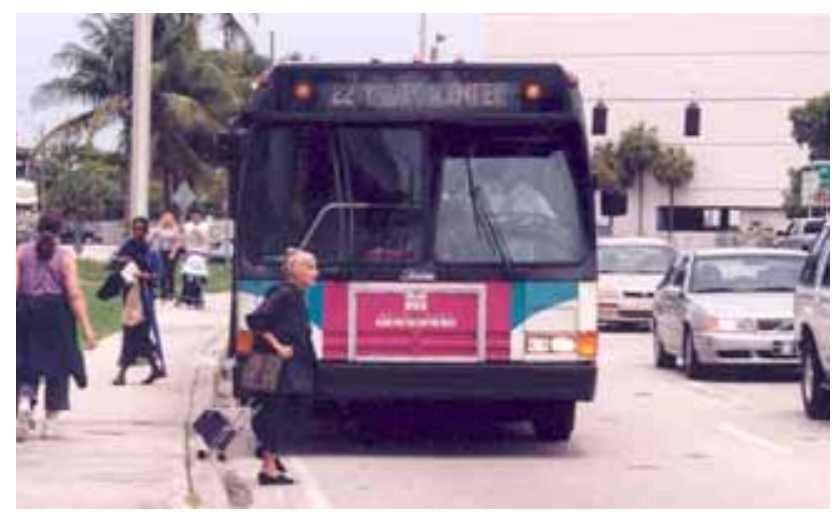

Figure 38. Pedestrian Conflicts With Bus and Traffic.

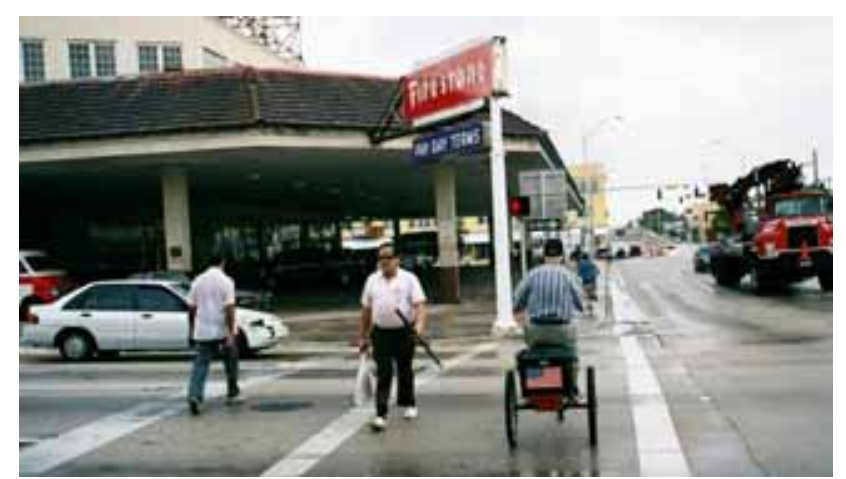

Figure 35. Pedestrians Violating Walk Signals.

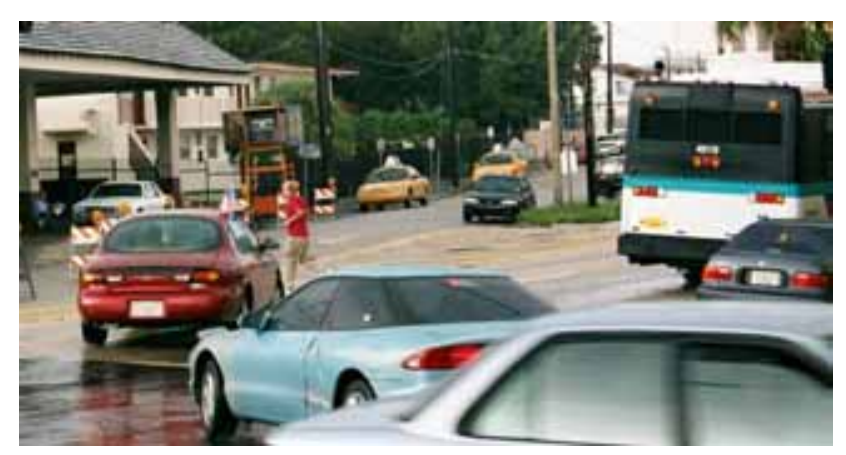

Figure 37. Pedestrian Conflicts With Turning Vehicles.

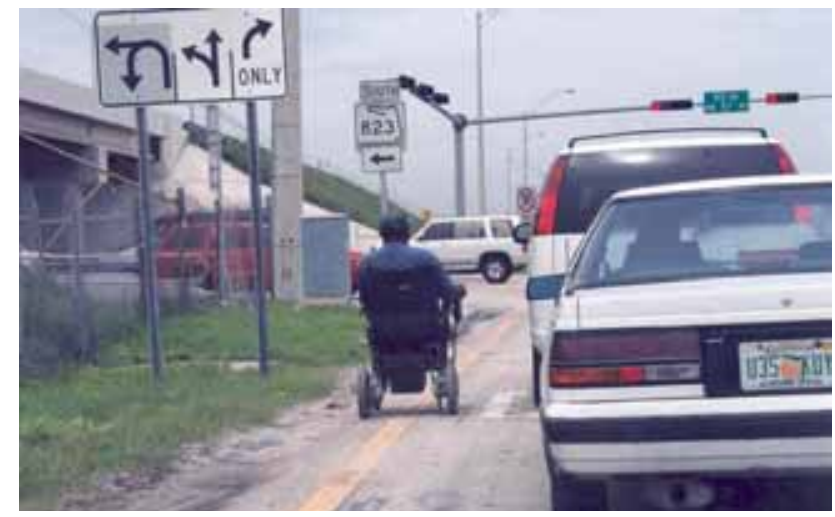

Figure 39. Missing Sidewalk Links and Lack of Facilities for Pedestrians. 


\section{CHAPTER 4: DESCRIPTION OF PEDESTRIAN SAFETY TREATMENTS}

Drawing on the pedestrian safety issues identified and discussed in Chapter 3, a total of 16 different pedestrian safety treatments were targeted to areas within Miami-Dade County, and particularly within the four selected zones (Little Haiti, Little Havana, Liberty City, and South Beach). A variety of education, engineering, and enforcement crash countermeasures were implemented over the course of the project. Countermeasure implementation began at different times and many have continued beyond the end point of the project. In addition to the NHTSAHSRC grant, countermeasures have been funded with Federal Section 163/402, Hazard Elimination, FDOT contributions, and other funds. NHTSA funds were spent in the following ways:

- GSA Printing and Reproduction: "Walking Through the Years" and other printed safety material, including development and reproduction of pedestrian safety posters for bus and rail transit vehicles.

- Safety Equipment: retro-reflective wrist wraps and zipper pulls;

- Engineering and Other Professional Services: preparation of data for GIS crash mapping;

- Travel and registration fees;

- Video supplies: dubbing and reproduction of "Walking Through the Years" video; and

- Project staff time: to guide the research study, determine countermeasures, conduct site visits, conduct evaluations, etc.

\section{Overview of Countermeasures}

Countermeasures were chosen by Miami-Dade County officials for each of the locations and zones, with input and consultation by the project team members. Specific educational measures were targeted toward appropriate age groups (e.g., children, senior citizens), and ethnic groups (e.g., Hispanic, Haitian, and English speakers) based on the characteristics of pedestrian crashes within each zone. The following is a summary of the various countermeasures that were implemented as part of the program:

\section{Education}

\section{Programs, Workshops, and Other Events}

- WalkSafe Program

- RYDER Trauma Center Classroom Education

- Pedestrian Safety Messages Mounted in Bus and Metrorail Train Cars

- Walk to School Day sponsored by SAFEKids Walk This Way

- Pedestrian Education by the Community Affairs Bureau of Miami-Dade Police Department

- Haitian Creole Elementary School and Older Pedestrian Safety Education Programs/Workshops 
Pedestrian safety presentations were made at more than 20 assemblies and older people's health fair events.

Brochures (see Figure 40)

- Walking Through the Years for Families brochure (English/Spanish)

- Walking Through the Years for Older Adults brochure (English)

- Walking Through the Years for Older Adults brochure (Spanish)

- Pedestrian, Walk Safely (English/Spanish)

- You and You Should Never Meet (turning vehicles)

- You and You Should Never Meet (visual screens)

- Haitian Heroes of Independence pedestrian safety cards (Haitian Creole - four versions)

- Safety Tips for Pedestrians (Haitian Creole)

Over 100,000 copies of the Walking Through the Years for Families brochure were distributed at "Walk to School Day" events in 2001-2005. About 60,000 copies of the "Walking Through the Years for Older Adults" brochure (English and Spanish) were distributed to mature pedestrians through senior centers and other public offices in and outside of the target zones. 

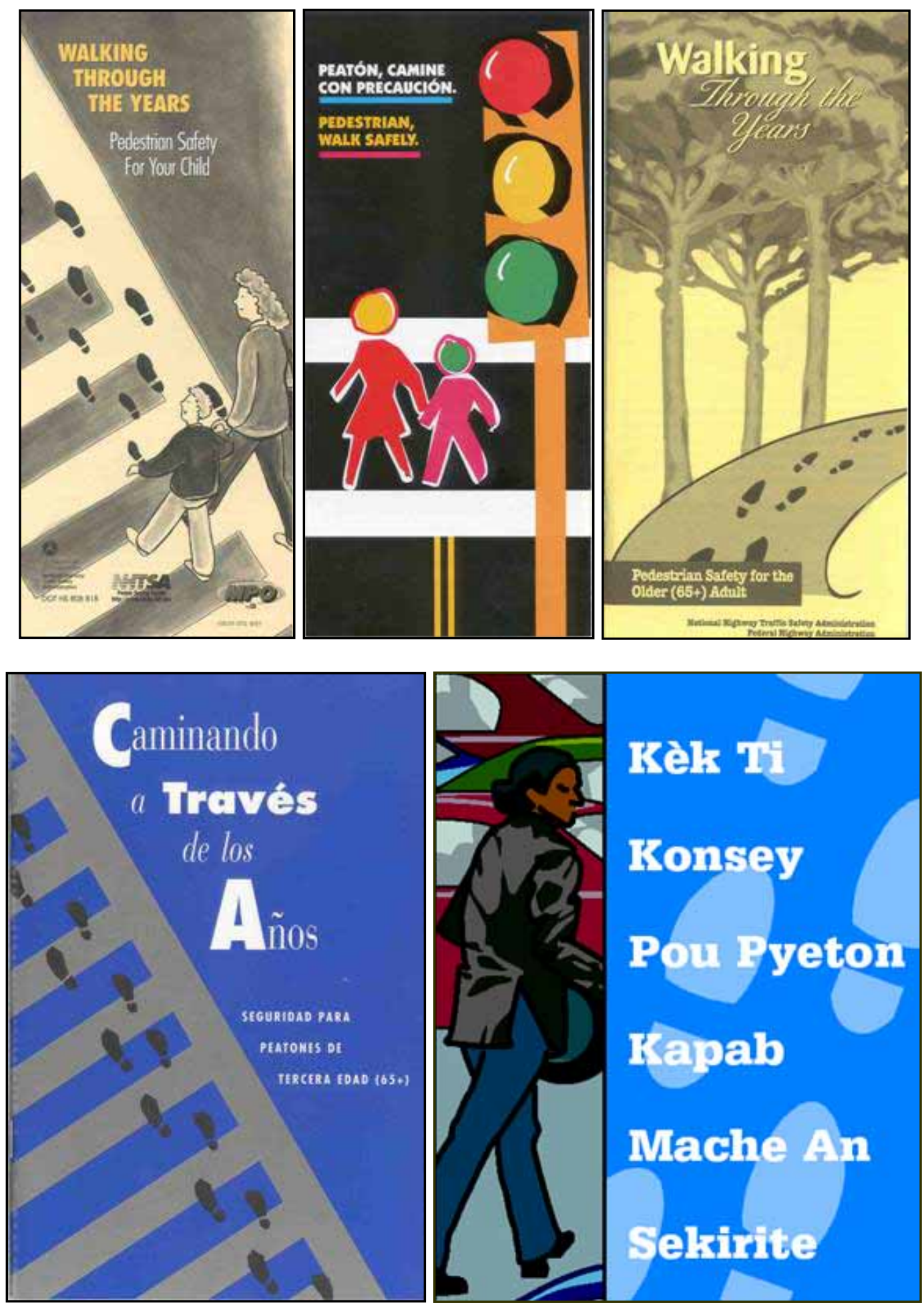

Figure 40. Examples of Educational Brochures. 
$\underline{\text { Videos }}$

- Walking Through the Years for Older Adults (English/Spanish/Haitian Creole)

- $\quad$ La Cita/The Date (Spanish; see Figure 41)

About 40 copies of "Walking Through the Years" video (dubbed in Spanish and Haitian Creole) were distributed to senior center and social service providers.

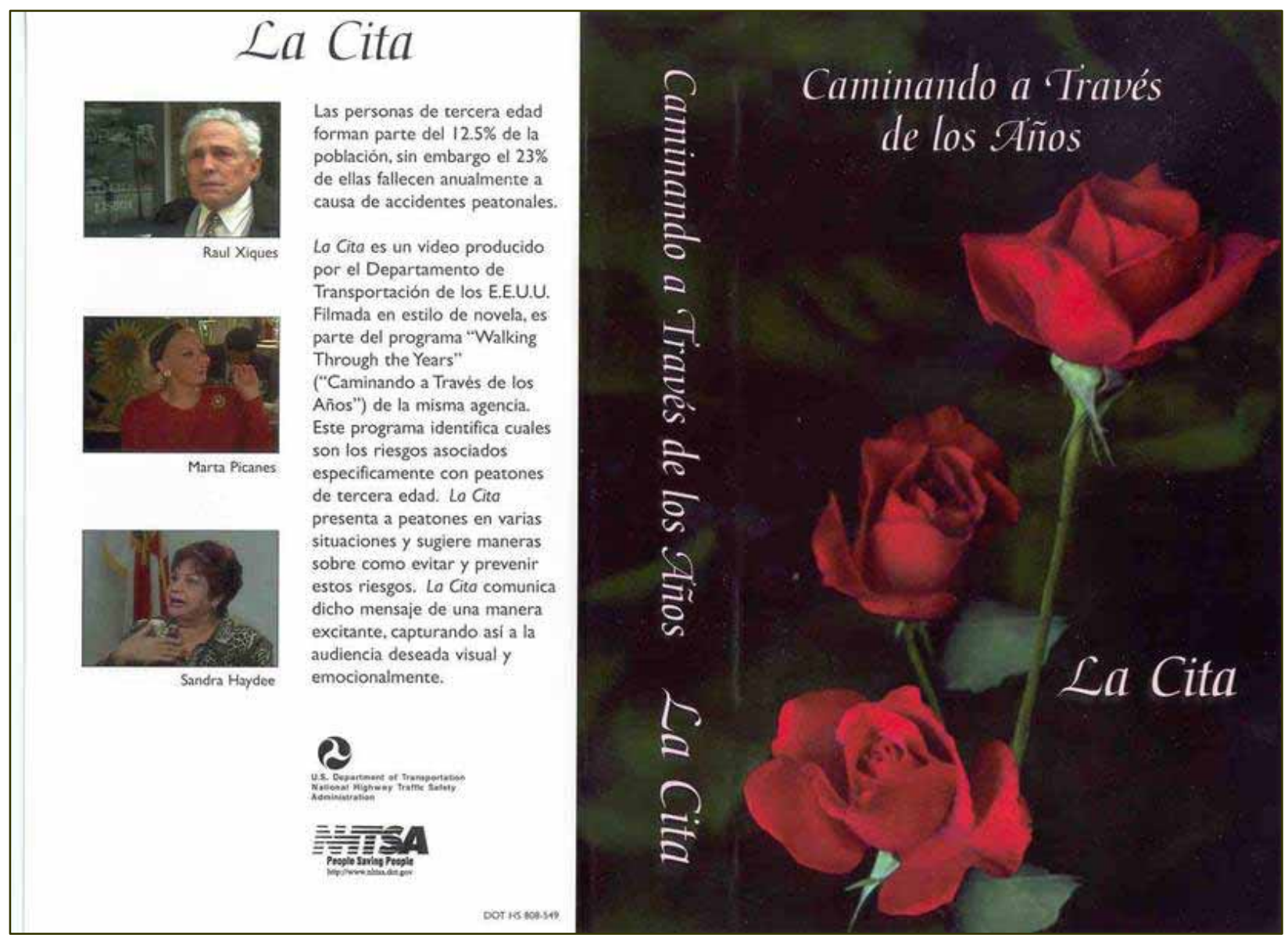

Figure 41. Example Video "La Cita."

$\underline{\text { Posters }}$

- Be Safe Be Bright (English)

- Think the Driver Can See You? (English)

- Walk Safe Miami (English/Spanish/Haitian Creole_-six versions, see Figure 42)

Pedestrian safety posters, many emphasizing pedestrian safety and visibility at night, were placed in some Miami-Dade Transit buses and trains, but the total number of posters placed is unknown. 

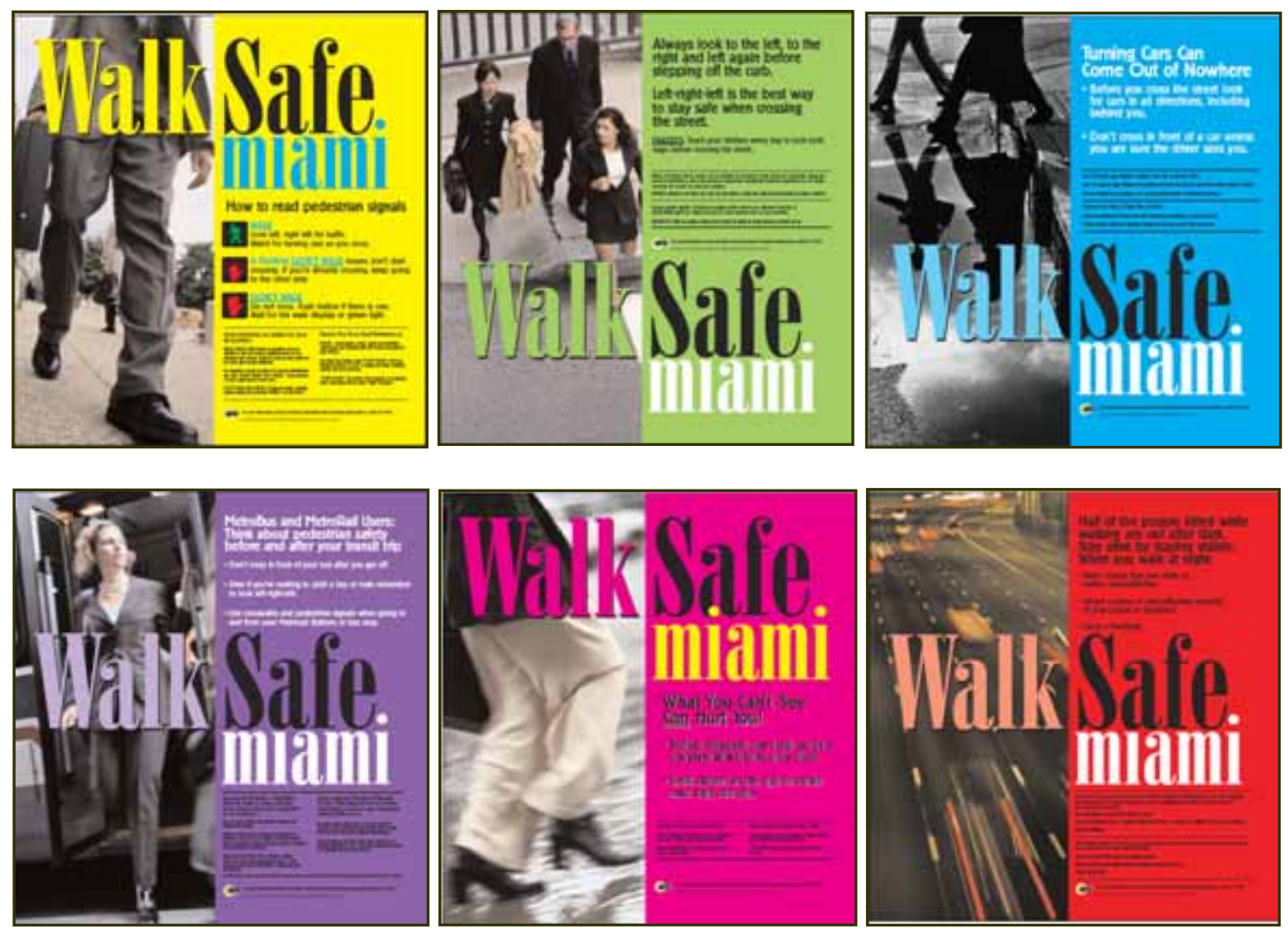

Figure 42. Example Walk Safe Miami Posters.

$\underline{\text { Giveaways }}$

- Retro-reflective zipper pulls

- Retro-reflective snap bracelets

- Retro-reflective wrist wraps

Around 10,000 retro-reflective zipper pulls and wrist wraps were distributed to students through schools. Additionally, 5,000 retro-reflective wrist wraps were distributed at elder health fair events.

\section{Enforcement}

- The Center for Education and Research in Safety, under a separate contract with NHTSA and the Florida Department of Transportation, conducted officer training in pedestrian safety enforcement at the City of Miami Beach Police Department. This enforcement program complemented other countermeasures in South Beach that were implemented during the Miami-Dade Demonstration project reported herein.

- Enforcement of Driver Yielding Program 


\section{Engineering}

Two engineering studies, the FDOT Engineering Project and the High Pedestrian Crash Corridor study were conducted. These studies identified 12 corridor segments with large numbers of pedestrian crashes and referred the locations to the Florida Department of Transportation for action through the Hazard Elimination Program. Over \$6.5 million in pedestrian safety projects were programmed or implemented on these corridors as a result.

Implementation began for many of these specific pedestrian safety treatments in early 2002, while other treatments were first implemented in late 2002 or in 2003. Some of the treatments continued for much of the implementation period. Others were tied to specific events (e.g., Walk to School annual events) or were ongoing (e.g., engineering treatments and distribution of educational posters on buses and safety education material distributed at senior centers and public buildings). A chart of the education timeline is given in Figure 43. At the end of this chapter is a matrix listing each countermeasure, its intended location and audience, and funding source (Table 6). This table provides a summary of each countermeasure installed, along with the type of treatment (education, enforcement, or engineering). The 16 education, enforcement, and engineering activities (some with several related sub-tasks) are discussed below in more detail. 


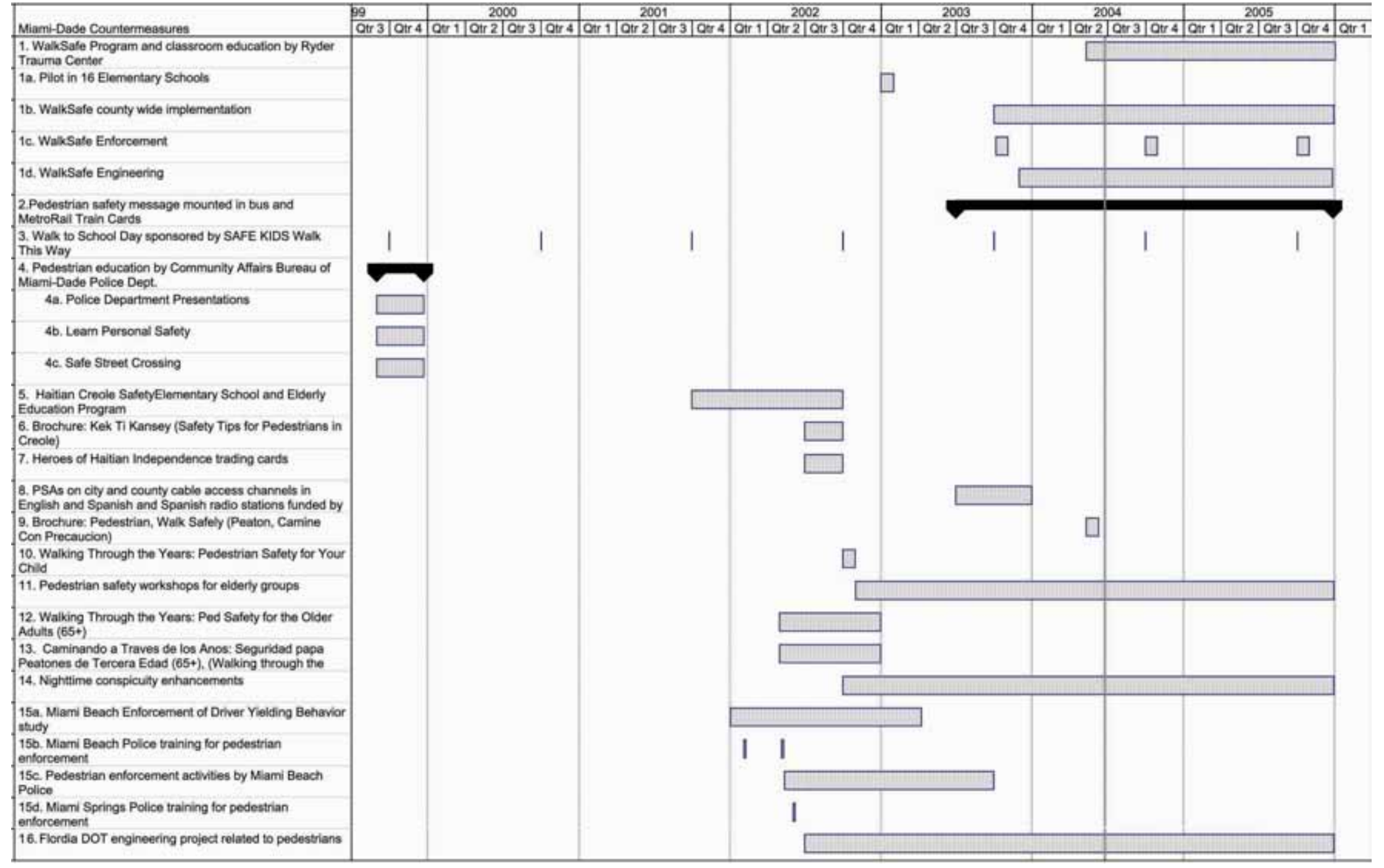

Figure 43. Countermeasure Implementation Timeline. ${ }^{2}$

\footnotetext{
${ }^{2}$ The black bars represent the total duration for activities with more than one sub-task. The gray bars represent the duration of each individual activity. Countermeasure 4 (a-c) was repeated each year.
} 


\section{Educational Countermeasures}

\section{WalkSafe Program and Ryder Trauma Center Classroom Education}

WalkSafe is a program, conducted by the University of Miami Ryder Trauma Center, aimed at reducing the incidence of children struck by vehicles by educating elementary school-age children and their teachers, parents, and communities about traffic safety. The program uses an educational training intervention, appropriate engineering countermeasures, and an enforcement component to help achieve its goal.

The WalkSafe Program educational training curriculum was pilot-tested and implemented in all 16 elementary schools in Liberty City in January 2003. The education component provides children in kindergarten through grade 5 with age-appropriate learning exercises and devices including videos, pedestrian skill practices and simulations, and poster contests. In April 2003, the Miami-Dade County Public School Board mandated that the program be given annually to students in kindergarten through grade 5 at all 220 elementary schools in Miami-Dade County. The program was shortened from five half-hour sessions presented on five consecutive days to three days as follows: Day 1-Education and videos, Day 2-Outside simulation/pretend road, and Day 3-Poster contest with pedestrian safety messages. The plan was to implement the program in October of each year to coincide with the International Walk to School Day. In October, 2003, the WalkSafe program was implemented in approximately $60 \%$ of all the public elementary schools in Miami-Dade County. A copy of the WalkSafe training course material may be found at: www.walksafe.us/walksafe/index.asp.

The program's law enforcement component included increased police patrols in the school zones around the 16 Liberty City pilot site elementary schools during October 2003 in efforts to deter speeding by way of police presence and issuing speeding citations. These patrols were expanded to all public elementary schools in the county in October 2004.

A host of engineering recommendations were made and several were implemented for each of the 16 elementary schools. Some recommendations fell under the jurisdiction of the Miami-Dade Public Works Department while others were the responsibility of the Miami-Dade County Public School system beginning in late 2003 and continuing through 2004. Changes made by the Public Works Department included installing a school speed zone flashing signal at one school and remarking worn pavement markings, and reinstalling signs at various schools. As part of the WalkSafe Program, several schools had their signs colored with engineering-grade yellow material replaced by signs containing new fluorescent yellow-green reflective material. However, a lack of funding prevented a full replacement at all schools of signs from the engineering-grade yellow to the new fluorescent materials.

\section{Pedestrian Safety Message Mounted in Bus and Metrorail Train Posters}

The Miami-Dade MPO produced six sets of different pedestrian education posters aimed at increasing pedestrian safety practices. The posters' safety messages were in English, Spanish, and Creole and covered pedestrian-related topics ranging from interpreting pedestrian signals and being visible at night to watching for turning cars and making eye contact with an oncoming 
driver before crossing the street. The posters were mounted in the county's 600 buses and most of the 135 MetroRail train cars free as a county public service. From July through September 2003, two of the six different posters were mounted in all of the county's buses. From October through December 2003 another two sets of posters were mounted along with half of the supply of the fifth set of posters. All posters remained installed unless they were vandalized or fell off due to humidity. Of the six sets of posters designated for the MetroRail train cars, one set was installed over the second half of 2003, and the second poster set was installed in May 2004.

\section{Walk to School Day Sponsored by SAFE KIDS Walk This Way}

In 1999, the National SAFE KIDS Campaign, supported by its program sponsor and partners, created the SAFE KIDS Walk This Way program to teach pedestrian safety to children and create safer, more walkable communities. The campaign sponsors Walk to School Day each October and supports the identification and correction of pedestrian problems near schools. The MiamiDade County SAFE KIDS Coalition spends approximately $\$ 2,600$ annually to purchase reflective zipper pulls and material that are distributed to the schools at no charge. The National SAFE KIDS Campaign provides banners, signs, pedestrian safety pamphlets and walkability surveys free to the coalitions.

Since 2002, thousands of students from seven Miami-Dade elementary schools and one middle school have participated. Additionally, a walkability assessment was conducted at the Morningside Elementary School, which resulted in the Miami-Dade Department of Public Works agreeing to install school crossing signs and restripe the crosswalks near the school.

\section{Pedestrian Education by the Community Affairs Bureau of the Miami-Dade Police Department}

The Pedestrian Safety Section of the Miami-Dade Police Department's Community Affairs Bureau makes numerous traffic safety presentations in schools throughout unincorporated Miami-Dade County. In addition to pedestrian safety, the staff covers such topics as seatbelt safety, bicycle safety, car seat safety, character development, and substance abuse. They use a variety of printed materials, including a 16-page 8 x 10 coloring book that provides traffic safety advice for the elementary school-age child and a four-page 8-1/2 x 11 activity/coloring booklet that teaches small children to stop at the curb and look left, right and left again before crossing the street even when a crossing guard is available to help. Pedestrian safety lessons are conducted for children in kindergarten through grade 3.

The Pedestrian Safety Section of the Miami-Dade police department helped the WalkSafe Miami program establish itself at the outset of the program. The WalkSafe Miami program developed its own curriculum and presentations, and over the course of the project sponsored numerous presentations and disseminated many of its own materials. This included police conducting speed enforcement and in-class educational programs, etc. Also, intensive school area safety plans were conducted in a phased approach at all elementary and middle schools (grades K through 8), first targeting schools in high-crash areas. 


\section{Haitian Creole Elementary School and Older Pedestrian Safety Education Programs}

Separate safety education programs were developed and presented to older residents and to elementary school children in Little Haiti. These programs were carried out by a former professor and graduate students at the University of Miami, as part of a pedestrian safety grant from FDOT. The older-pedestrian program was presented in Haitian Creole; the program for elementary school children was presented in English. The development of the program for older pedestrians started with a focus group comprised of 24 older pedestrians and served to identify the difficulties that they encounter while crossing streets in Little Haiti and their perceptions of measures that could be taken to improve pedestrian safety. Two workshops were conducted with older pedestrians - the first with 41 attendees and the second with 60-emphasizing dangerous intersection avoidance, backing crashes, and being conspicuous. An intersection simulation provided the participants with an opportunity to practice their intersection-crossing skills.

The Elementary School Program consisted of four 45-minute workshops conducted at three elementary schools. The programs reached 389 elementary school children, primarily from the third and fourth grades. In addition to behaviors related to walking, safety behaviors related to bicycling, skating, and riding in private vehicles and on the school bus were also covered. As part of the program, children were shown a video on safety titled "Getting to School the Safe Way." Pedestrian issues focused on crossing at intersections and crossing between parked cars.

Both programs ran from October 2001 through September 2002 and were supported by radio advertisements and by postings on Haiti Online - a Haitian Web site that attracts a large audience, including residents of Little Haiti. The programs also were supported by a brochure in Haitian Creole that provides pedestrian safety advice and by four Haitian Creole trading cards, which depict heroes of Haitian independence on one side and provide pedestrian safety tips on the other side (see below).

\section{Brochure: Safety Tips for Pedestrians in Haitian Creole}

Kek Ti Konsey Pou Pyeton Kapab Mache An Sekirite (Safety Tips for Pedestrians) is a 4-inch by $51 / 8$-inch color brochure in Haitian Creole that provides pedestrian safety advice to adults. The brochure includes precautions to take before and while crossing the street and crossing in front of and behind cars. Distribution of the cards began in July 2002 and the supply only lasted several months (it is not known how many cards were officially distributed).

\section{Heroes of Haitian Independence Trading Cards}

Four 2-inch by 3 1/2-inch cards each depict a hero of Haitian independence on one side and provide pedestrian safety tips on the other (see Figure 44). The cards' text is in Haitian Creole and their safety messages cover tips for reading a pedestrian signal, crossing the street, walking at night, and driver responsibility toward pedestrians. Distribution of the cards began in July 2002 and the supply only lasted several months. 


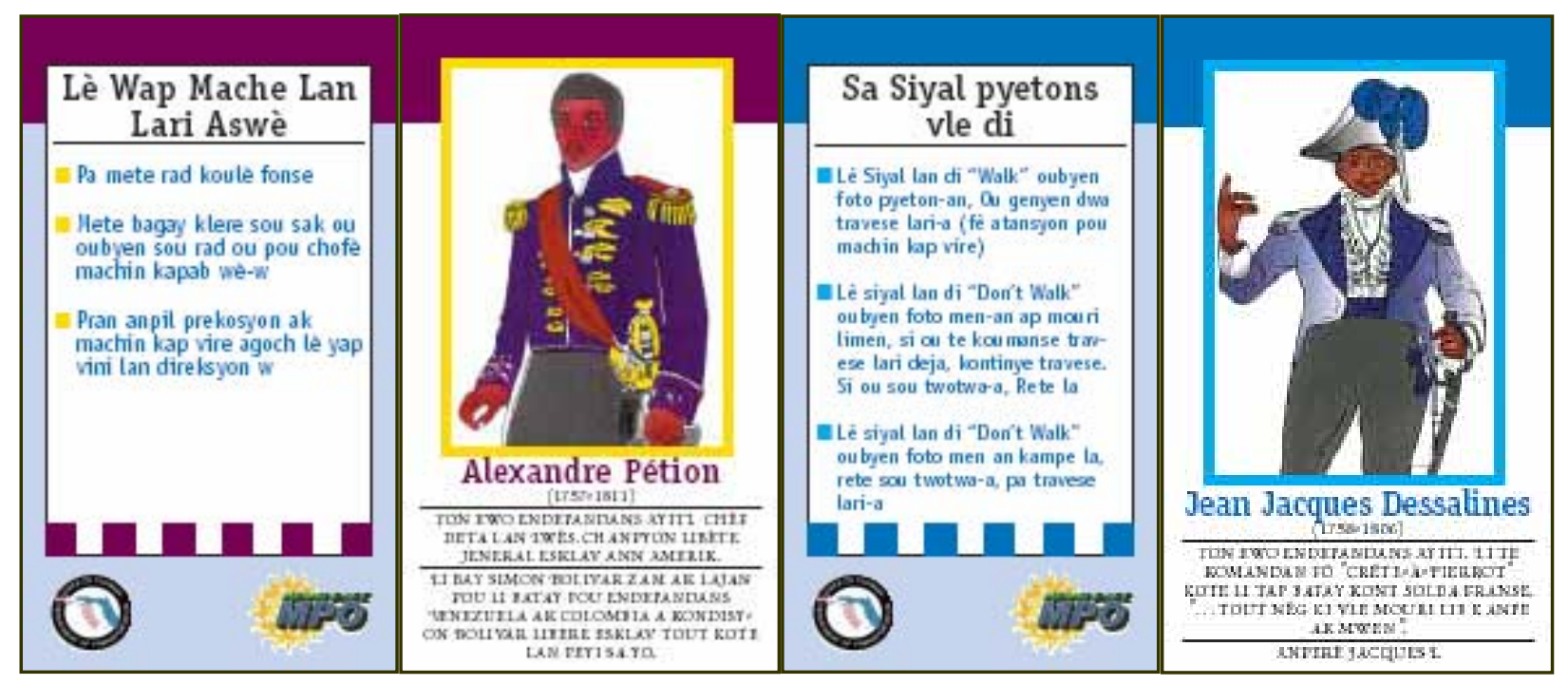

Figure 44. Heroes of Haitian Independence Trading Cards.

\section{Public Service Announcements Played on City and County Cable Access Channels and Radio Stations}

From April 2003 through December 2003, PSAs about pedestrian safety were distributed and broadcasted on city and county access channels in Spanish and English and on selected Spanishspeaking radio stations.

\section{Brochure: Pedestrian, Walk Safely}

A brochure titled Pedestrian, Walk Safely (Peaton, Camine Con Precaucion) (DOT HS 808 166) is a four-fold 4 -inch by $81 / 2$-inch color brochure providing families with the pedestrian safety advice in both English and Spanish. Brochures were delivered to organizations and institutions in Miami-Dade County, Liberty City, and Little Havana such as the Miami-Dade School Board, hospital, public library, police departments, and elected officials' offices. Distribution of the brochures to the public began in October 2002 and continued throughout the project.

\section{Walking Through the Years: Pedestrian Safety for Your Child}

Walking Through the Years : Pedestrian Safety for Your Child (Caminando a Traves de los Anos: Seguridad Peatonal Para Ninos) (DOT HS 808 818) is a four-fold 4-inch by 9-inch twocolor brochure providing safety guidelines to parents and caregivers to help protect children from pedestrian crashes. The brochure is written in both English and Spanish and conveys facts on common locations, times, and circumstances associated with child pedestrian crashes; streetcrossing skills parents can practice to set good examples for their children; and pedestrian safety tips to teach children. Brochures were delivered to a range of organizations and institutions in Miami-Dade County, Liberty City, and Little Havana such as the Miami-Dade School Board, hospital and medical departments, public libraries, police departments, and elected officials' offices. Distribution of the brochures to the target population began in June 2002 and continued throughout the project. 


\section{Pedestrian Safety Workshops for Older Populations}

The Miami-Dade MPO pedestrian-bicycle coordinator began providing workshops on pedestrian safety to older pedestrians and groups working with older populations in November 2002 (see Figure 45). The workshops were an ongoing component of the project.

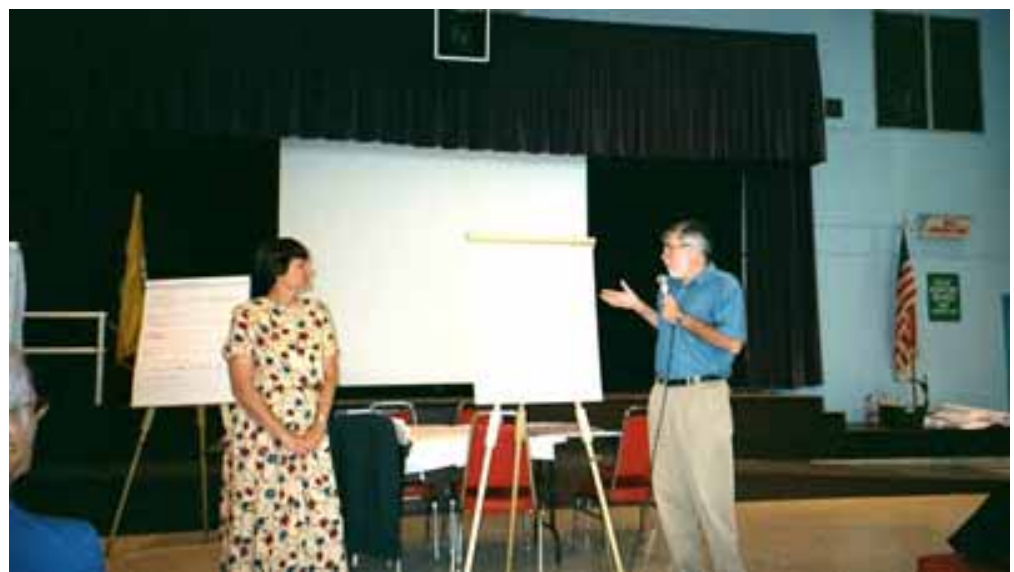

Figure 45. Pedestrian Safety Workshop for Older Pedestrians.

\section{Walking Through the Years: Pedestrian Safety for the Older Adult}

Walking Through the Years: Pedestrian Safety for the Older Adult (65+) (DOT HS 809 083) is a 12-page, 4-inch by 9-inch booklet prepared for adults 65 and older and for implementers of programs for older adults. The booklet documents the pedestrian problems and major risks faced by this population along with advice to manage each risk. Brochures were delivered to organizations and institutions in Miami-Dade County, Miami Beach and Little Havana such as the Miami-Dade School Board, hospital and medical departments, retirement homes, public libraries, police departments, and older affairs and elected officials' offices. Distribution of the 60,000 brochures to the target population began in June 2002 and ended in December 2002.

\section{Caminando a Traves de los Anos: Seguridad para Peatones de Tercera Edad (65+)}

This is a 20-page 5 1/2-inch by 8 1/2-inch booklet (DOT HS 808 515) in Spanish prepared for implementers of pedestrian programs for adults 65 and older. The document describes the $65+$ pedestrian problem, major risks, and advice for each risk. Brochures were delivered to organizations and centers in Miami-Dade County, Miami Beach, and Little Havana, such as the Miami-Dade School Board, hospitals, retirement homes, public libraries, police departments, and older affairs and elected officials' offices. Distribution of the brochures to the public began in June 2002 and ended in December 2002.

\section{Nighttime Conspicuity Enhancements}

More than 400 posters on nighttime conspicuity related to pedestrian safety were distributed to organizations to display in public buildings. 


\section{Enforcement Countermeasures}

\section{Enforcement of Driver Yielding Behavior Study, Two Police Pedestrian Safety Training}

Programs, and Enforcement

Van Houten and Malenfant (2003) conducted a study of driver yielding behavior at four crosswalks in each of two - an east and west - high-crash corridors in the City of Miami Beach. Police teams were situated at eight selected crosswalks. Each team included a decoy pedestrian who crossed the street (see Figure 46) when other pedestrians were not present and a spotter who radioed failure-to-yield violations to other officers who flagged the violators and gave them a verbal warning or citation and an enforcement flyer.

The police stopped 1,562 motorists for failing to yield to pedestrians over the period of a year, with 1,218 of these stopped during the first two weeks of the program (see Figure 47). Three hundred seven citations were issued, of which 188 were given during the first eight weeks of the program. At baseline, 3.3\% and $18.2 \%$ of the drivers yielded to pedestrians in the west and east corridors respectively. The introduction of the enforcement program at the four sites in the west corridor led to an increase in yielding to $27.6 \%$ during the first week of the program while no increase in yielding occurred at the untreated east corridor. The introduction of the enforcement operations in the east corridor led to an increase in yielding to $28.8 \%$ in this corridor, while increased yielding was maintained in the west corridor. Monthly follow-up data indicated that the gains produced by the program were maintained in the absence of high levels of police enforcement with overall yielding rates of $27.8 \%$ in the west corridor and $34.1 \%$ in the east corridor during the follow-up data collection (Van Houten \& Malenfant, 2003).

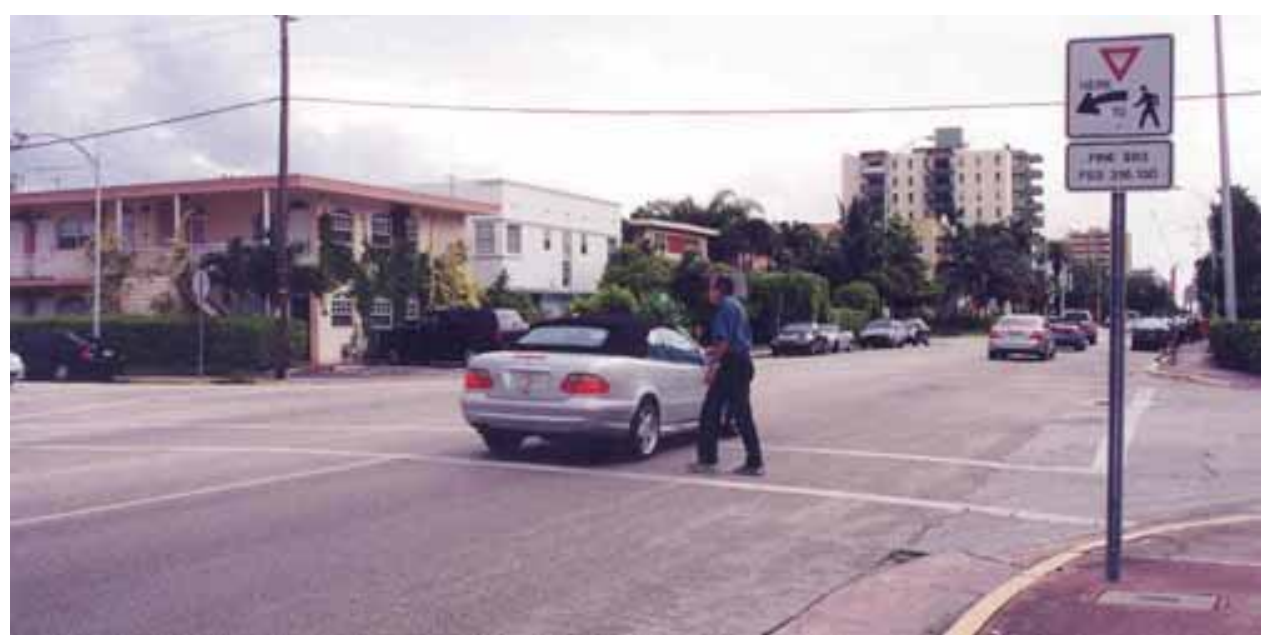

Figure 46. “Decoy” Pedestrian Crossing the Street in Enforcement Operation. 


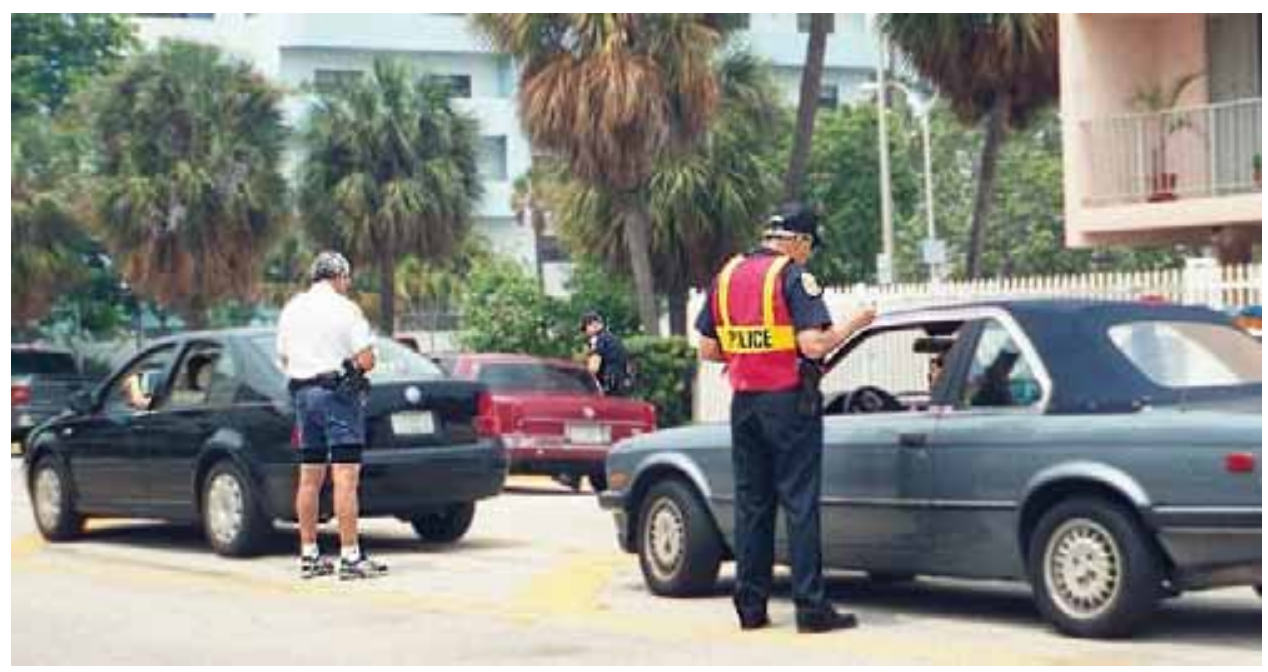

Figure 47. Enforcement Operations in Miami-Dade.

Additionally, in 2002 police officers in Miami Beach and Miami Springs (eight officers) received additional training on pedestrian safety and enforcement activities to address common violations and behaviors that lead to collisions between pedestrians and motor vehicles. Police then conducted a number of enforcement operations; however, the total number of citations and warnings given is not known.

\section{Engineering Countermeasures}

\section{FDOT Engineering Projects Related to Pedestrians}

During the implementation period of January 2002 through December 31, 2004, numerous engineering and roadway treatments were implemented by FDOT and Miami-Dade County. These included measures such as adding raised medians on selected multilane roads, installing missing sidewalk links, installing pedestrian warning signs (see Figure 48) at specific locations, revising traffic signal timing, implementing safer facilities in selected school zones, and others. Many of these treatments correspond to the normal types of road improvements that have been ongoing in Miami-Dade County in recent years in an effort to improve pedestrian safety. During the course of the project, members of the project team conducted numerous site inspections with county, MPO, and FDOT officials and developed recommendations for pedestrian safety improvements. Such site inspections were conducted throughout the four high-crash zones. 

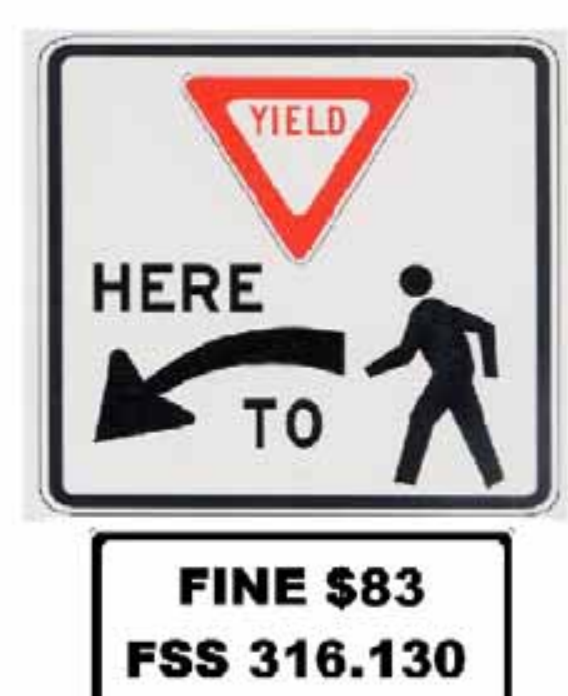

TURNING VEHICLES

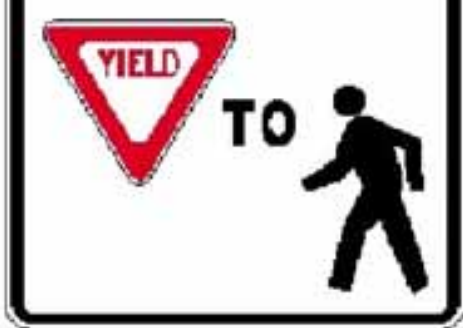

FINE \$83

FSS 316.075

Figure 48. Signs for Drivers to Yield to Pedestrians.

Table 6 lists the countermeasures for Miami-Dade previously described and identifies the location, intended audience and funding source of each countermeasure. For countermeasures without a funding source listed, the information was unknown. 
Table 6. Countermeasure Locations in Miami-Dade County.

\begin{tabular}{|c|c|c|c|c|c|c|c|c|c|}
\hline \multirow[t]{2}{*}{ Countermeasure } & \multicolumn{3}{|c|}{ Location } & \multicolumn{3}{|c|}{$\begin{array}{c}\text { Countermeasure } \\
\text { Type } \\
\end{array}$} & \multirow[t]{2}{*}{$\begin{array}{c}\text { Target } \\
\text { Audience }\end{array}$} & \multirow[t]{2}{*}{$\begin{array}{l}\text { Funding } \\
\text { Source (s) }\end{array}$} & \multirow[t]{2}{*}{ Implementation Period } \\
\hline & ڤี & & : & : & 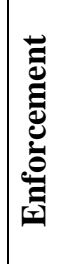 & 番 & & & \\
\hline $\begin{array}{l}\text { 1. WalkSafe program and Ryder Trauma Center } \\
\text { classroom education }\end{array}$ & & & $\mathrm{X}$ & $\mathrm{X}$ & & & $\mathrm{C}$ & $\begin{array}{l}\text { FDOT/NHTSA } \\
\text { sub to } \mathrm{M}-\mathrm{D}\end{array}$ & $1 / 03-12 / 05$ \\
\hline $\begin{array}{l}\text { 2. Pedestrian safety message mounted in bus and } \\
\text { MetroRail train card }\end{array}$ & $\mathrm{X}$ & & & $\mathrm{X}$ & & & $\mathrm{P}$ & $\begin{array}{l}\text { NHTSA and } \\
\text { M-D }\end{array}$ & $7 / 03-12 / 05$ \\
\hline $\begin{array}{l}\text { 3. Walk to School Day sponsored by SAFE KIDS } \\
\text { Walk This Way }\end{array}$ & & & & $\mathrm{X}$ & & & $\mathrm{C}$ & $\begin{array}{l}\text { National Safe } \\
\text { SAFE KIDS } \\
\text { Campaign } \\
\end{array}$ & Annual Event in October \\
\hline $\begin{array}{l}4 \text { Pedestrian education by the Community Affairs } \\
\text { Bureau of the Miami-Dade Police Department } \\
\text { a) Police Dept. Presentation } \\
\text { b) Safe Street-Crossing booklet } \\
\text { c) Learn Personal Safety booklet }\end{array}$ & & & & $\mathrm{X}$ & & & $\mathrm{C}$ & M-D PD & Ongoing periodically \\
\hline $\begin{array}{l}\text { 5. Haitian Creole Elementary School and Older } \\
\text { Pedestrian Safety Education Programs }\end{array}$ & & & & & & & $\begin{array}{l}\mathrm{H} \\
\mathrm{C} \\
\mathrm{O}\end{array}$ & $\begin{array}{l}\text { 163/402 FDOT } \\
\text { Safety Grant }\end{array}$ & Mostly in 2002 \\
\hline $\begin{array}{l}\text { 6. Brochure: Safety Tips for Pedestrians in Haitian } \\
\text { Creole }\end{array}$ & $\mathrm{X}$ & & $\mathrm{X}$ & & & & $\mathrm{H}$ & FDOT & $7 / 02-9 / 02$ \\
\hline 7. Heroes of Haitian Independence trading cards & $\mathrm{X}$ & $\mathrm{X}$ & $\mathrm{X}$ & $\mathrm{X}$ & & & $\mathrm{H}$ & $\mathrm{MPO} / \mathrm{PD}$ & $7 / 02-9 / 02$ \\
\hline $\begin{array}{l}\text { 8. Public service announcements played on city and } \\
\text { county cable access channels and radio stations }\end{array}$ & & & & $\mathrm{X}$ & & & $\begin{array}{l}\mathrm{P} \\
\mathrm{D}\end{array}$ & M-D/NHTSA & $4 / 03-12 / 03$ \\
\hline 9. Brochure: Pedestrian, Walk Safely & & $\mathrm{X}$ & $\mathrm{X}$ & $\mathrm{X}$ & & & $\mathrm{P}$ & FDOT & $6 / 04$ \\
\hline
\end{tabular}




\begin{tabular}{|c|c|c|c|c|c|c|c|c|c|}
\hline \multirow[t]{2}{*}{ Countermeasure } & \multicolumn{3}{|c|}{ Location } & \multicolumn{3}{|c|}{ Countermeasure } & \multirow{2}{*}{$\begin{array}{c}\text { Target } \\
\text { Audience }\end{array}$} & \multirow{2}{*}{$\begin{array}{c}\text { Funding } \\
\text { Source (s) }\end{array}$} & \multirow[t]{2}{*}{ Implementation Period } \\
\hline & صَّ & 焉 & 吾 & 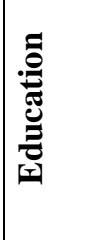 & 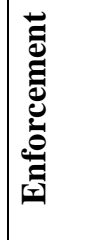 & 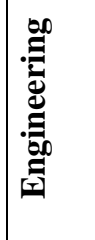 & & & \\
\hline $\begin{array}{l}\text { 10. Walking Through the Years : Pedestrian Safety for } \\
\text { Your Child }\end{array}$ & & & $\mathrm{X}$ & $\mathrm{X}$ & & & C, A & FDOT & $7 / 02$ \\
\hline 11. Pedestrian Safety Workshops for Older groups & $X^{X}$ & $\mathrm{X}$ & $\mathrm{X}$ & $\mathrm{X}$ & $\mathrm{X}$ & & $\mathrm{O}$ & $\begin{array}{l}\text { Could be project } \\
\text { or } \\
\text { M-D funds }\end{array}$ & $10 / 02-12 / 05$ \\
\hline $\begin{array}{l}\text { 12. Walking Through the Years: Pedestrian Safety for } \\
\text { the Older Adult }\end{array}$ & $\mathrm{X}$ & & $\mathrm{X}$ & $\mathrm{X}$ & & & $\mathrm{O}$ & FDOT & $4 / 02-12 / 02$ \\
\hline $\begin{array}{l}\text { 13. Caminando a Traves de los Anos: Seguridad para } \\
\text { Peatones de Tercera Edad }(65+)\end{array}$ & $\mathrm{X}$ & & $\mathrm{X}$ & $\mathrm{X}$ & & & $\mathrm{H}$ & FDOT & $4 / 02-12 / 02$ \\
\hline $\begin{array}{l}\text { 14. Nighttime Conspicuity Enhancements (reflective } \\
\text { bands and posters) }\end{array}$ & $X$ & $X$ & $\mathrm{X}$ & $\mathrm{X}$ & & & $\mathrm{P}$ & & $10 / 02-12 / 05$ \\
\hline $\begin{array}{l}15 \text { a) Enforcement of Driver Yielding Behavior study } \\
\text { b) Miami Beach Police training for ped. enforcement } \\
\text { c) Miami Beach Police enforcement activities } \\
\text { d) Miami Springs Police training for ped. } \\
\text { enforcement }\end{array}$ & $\mathrm{X}$ & & & $\mathrm{X}$ & & & $\begin{array}{l}\mathrm{D} \\
\mathrm{P}\end{array}$ & $\begin{array}{l}\text { a) M-D/NHTSA } \\
\text { b)M-D/Miami } \\
\text { Beach PD }\end{array}$ & $1 / 02-3 / 03$ \\
\hline 16. FDOT engineering project related to pedestrians & & & & & & $\mathrm{X}$ & $\begin{array}{l}\mathrm{P} \\
\mathrm{D}\end{array}$ & $\begin{array}{l}\text { Federal Hazard } \\
\text { Elimination }\end{array}$ & $7 / 02-12 / 05$ \\
\hline \multicolumn{10}{|c|}{$\begin{array}{l}\text { Key: M-D=Miami-Dade; NHTSA=Current NHTSA Contract; FDOT=Florida Department of Transportation; FHWA=Federal Highway Administration; } \\
\text { PD=Police Department }\end{array}$} \\
\hline
\end{tabular}




\section{CHAPTER 5: EVALUATION RESULTS}

This chapter reports on two separate sets of analyses carried out to determine the effectiveness of the pedestrian safety interventions implemented in Miami-Dade. The first was a countywide analysis comparing trends in Miami-Dade pedestrian crash rates with those in three comparison populations: neighboring Broward County, six other Florida metropolitan counties, and statewide. The second analysis utilized special analysis tools to examine changes in crash densities (pedestrian crashes per square mile) within each of the four zone areas targeted by the project: South Beach, Little Haiti, Little Havana, and Liberty City. The analysis also includes a descriptive examination of changes in specific types of crashes observed in each of the zone areas.

\section{Countywide Crash Analysis}

As described in greater detail in Chapter 3, all motor vehicle crashes occurring in Florida during the years 1996 to 2004 in which at least one pedestrian was involved were obtained from the official Florida Department of Highway Safety and Motor Vehicles database. Crashes in MiamiDade County were identified for analysis purposes by the county code for each crash. In other words, this database represented all of the computer crash records coded as pedestrian related in Miami-Dade County during the study period of January 1, 1996 through December 31, 2004.

During this 9-year time period, 79,887 pedestrian-related crashes occurred statewide, of which $17,308(21.7 \%)$ were in Miami-Dade County. The vast majority of pedestrian-related crashes involved only one pedestrian $(76,255 ; 95.4 \%)$. The distribution of crash times is illustrated in Figure 49. The distribution of crashes by hour of day was almost bell-shaped with the largest representation of pedestrian crashes occurring during daylight hours. The largest proportions of pedestrian crashes during the study period occurred from afternoon to evening hours, about 2 to 8 p.m. There was also a morning peak from 7 to 9 a.m. 


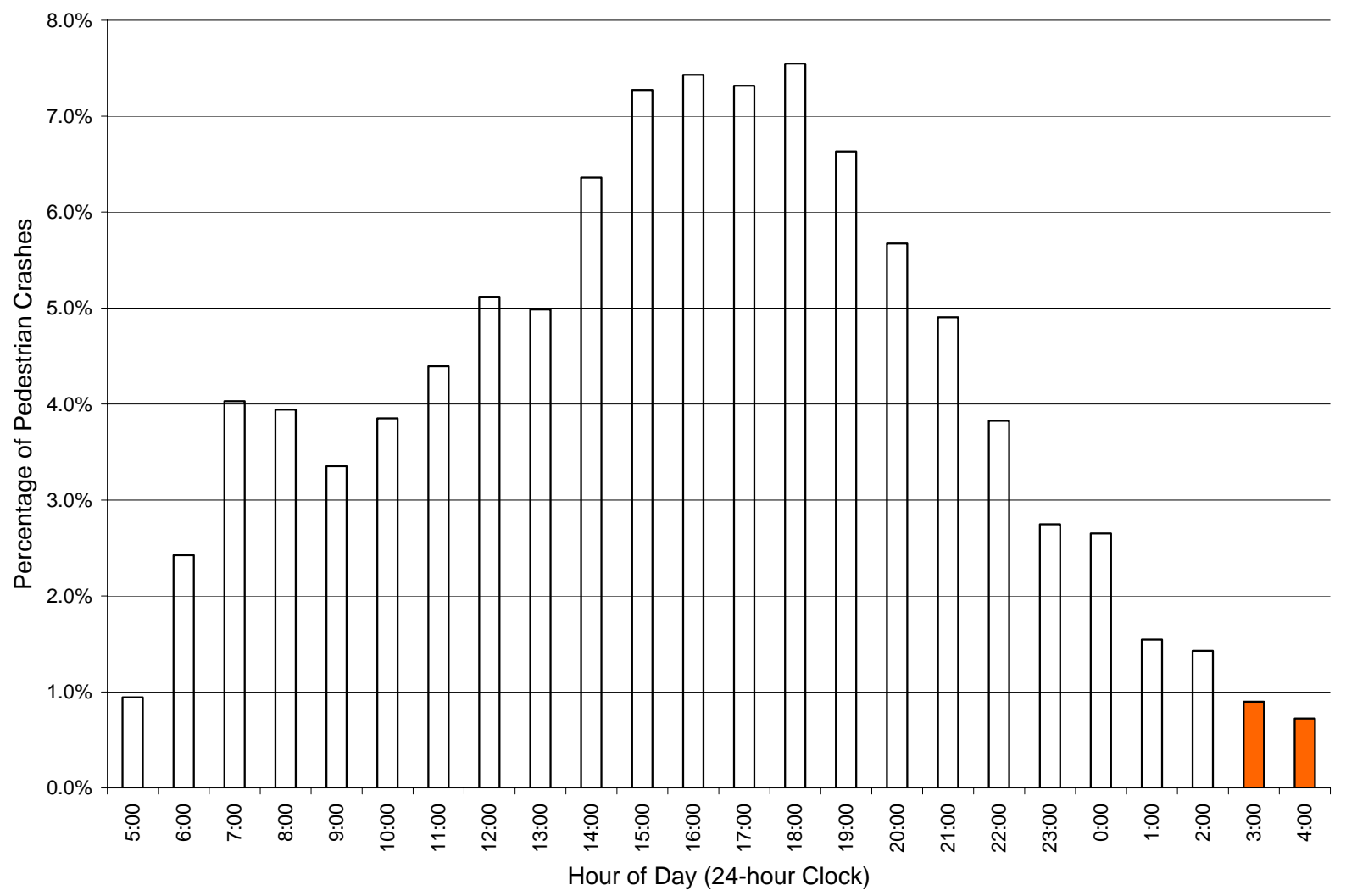

Figure 49. Statewide Percentage Distribution of Pedestrian Crashes in Florida by Hour of Day.

In assessing the impact of the pedestrian safety project implemented in Miami-Dade County, several other jurisdictions were chosen to serve as control groups to remove the effects of preexisting downward trends and other changes in Florida pedestrian crash rates that could otherwise be mistaken for program effects. Given the metropolitan nature of Miami-Dade County, it seemed most fair to compare changes in pedestrian crash rates in this county to those of other metropolitan Florida counties. For this purpose, neighboring Broward County was used as a control group. Broward County was selected as a stand-alone control group because it is the county directly north of Miami-Dade County and has many similarities (e.g., highly populated, borders the Atlantic Ocean, large percentage of retirees and vacationers, similar street system, etc.), although there are differences in racial/ethnic makeup. Broward County did not get the pedestrian safety program that was implemented in Miami-Dade County, so it was an appropriate area to use as a control group. However, because Broward County is adjacent to Miami-Dade, it was possible that some effects of the Miami-Dade pedestrian projects could have spilled over into Broward County that could bias such comparisons. (Note: Because of the focused nature of the countermeasures in Miami-Dade County, any possible spillover effects into neighboring Broward County were thought to be minor, if any.) Furthermore, if there were, in part, any spillover effects of the treatments into Broward County, the use of Broward County as a control group would result in underestimating the full effects of the Miami-Dade treatments. Therefore, another control group consisting of the six metropolitan counties in Florida (i.e., Duval, 
Hillsborough, Pinellas, Palm Beach, Orange, and Broward County) was also used. A final control group consisted of all Florida pedestrian crashes, except those in Miami-Dade County. The monthly numbers of pedestrian crashes in Miami-Dade County, Broward County, the six Florida metropolitan counties, and statewide (excluding Miami-Dade County) during 1996 to 2004 are shown in Figure 50. For ease in interpreting the trends, the 12-month moving average of each series is also shown.

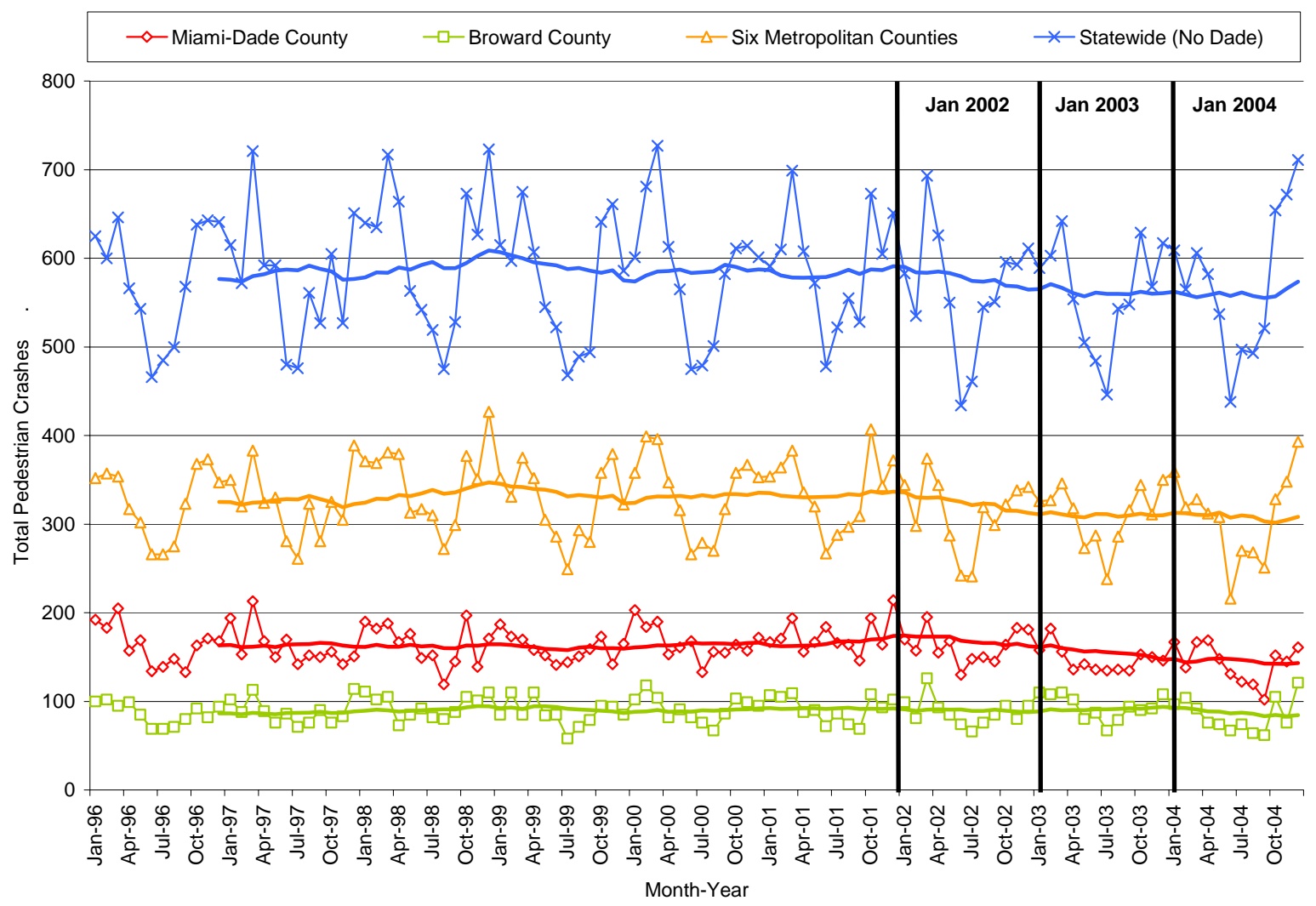

\section{Figure 50. Monthly Numbers of Pedestrian Crashes in Miami-Dade County, Broward County, Six Metropolitan Counties (Combined), and Statewide From 1996-2004.}

From the raw counts of pedestrian crashes shown in Figure 50, it is apparent that the absolute number of pedestrian crashes decreased in all series, with the possible exception of Broward County subsequent to January 2002. These counts are somewhat misleading because during the time period shown, there were also changes in the underlying populations of people who could contribute to pedestrian crashes. In particular, the population in Florida was increasing, which would be expected to be associated with higher absolute numbers of pedestrian crashes. To account for changes in the underlying population, the monthly counts were turned into rates per 100,000 population. Appendix B provides monthly crash and population data. The monthly total pedestrian crash rates are illustrated in Figure 51. Again, the 12-month moving average in each series is also shown to help with interpreting or identifying the trends. 


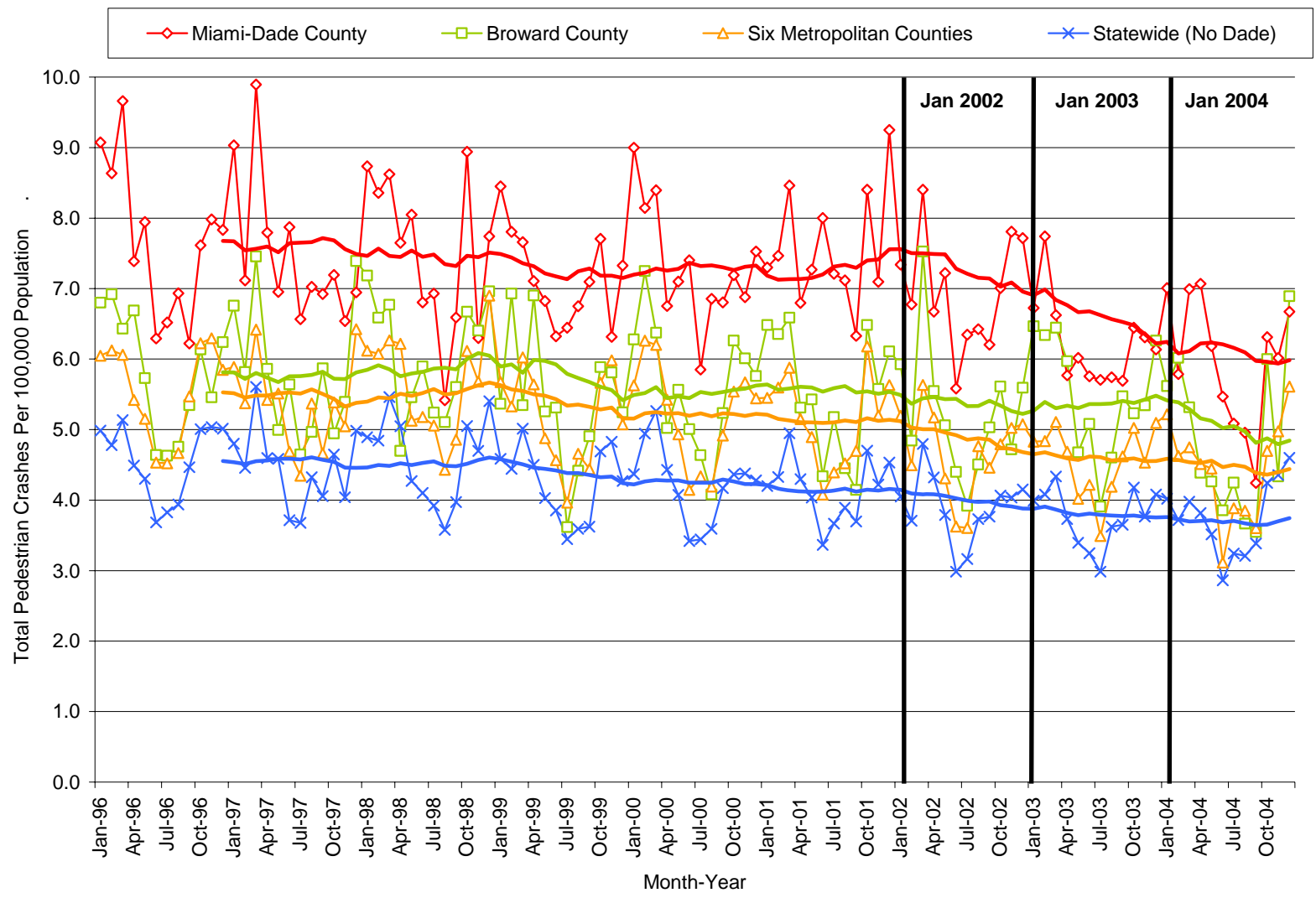

Figure 51. Monthly Pedestrian Crashes per 100,000 Population in Miami-Dade County, Broward County, Six Metropolitan Counties (Combined), and Statewide From 1996-2004.

It is apparent from the figure that Miami-Dade had higher pedestrian crash rates than any of the other series, including the rest of Florida. The decrease in pedestrian crashes in Miami-Dade County is much more apparent in the per-capita figure, and it does appear to coincide with the time period during which the pedestrian safety program was underway. However, also apparent in the other control series are downward trends in pedestrian crashes that began some time before the interventions in Miami-Dade County. This downward trend is also apparent in the MiamiDade County series, but is much more gradual than the sharp decrease in the pedestrian crash rate that began in early 2002. It is unknown why pedestrian crash rates were slowly decreasing in Florida during the time period shown, but some evidence shows that the drop could partly be a sign of decreasing pedestrian activity. Census data show that from 1990 to 2000, the percent of people walking to work dropped from $2.51 \%$ to $1.71 \%$ in Florida. In Miami-Dade County, the percent of people walking to work dropped from 2.53 to $2.15 \%$. It is important to remove this trend from the Miami-Dade County series before evaluating the effect of the interventions. This is the reason that the control series were included in the analyses. Also note that the pedestrian crash rate in Miami-Dade actually appears to have leveled off in early 2001 and then began to increase in the later half of 2001. At this point there is a bump in the pedestrian crash rate. A similar bump can be seen in the Broward County series around January 2003. In other words, the pedestrian crash rate in Miami-Dade County was increasing in late 2001 before the countermeasure program was implemented, beginning in early 2002. 


\section{Overall Impact of Pedestrian Safety Program}

To determine the overall impact of the pedestrian safety program, multivariate intervention ARIMA time series analysis was used (Box \& Jenkins, 1970; Box \& Tiao, 1975). Based on the timeline in which interventions were implemented in Miami-Dade County (see Figure 43), three different intervention points were tested in each model: (a) January 2002, (b) January 2003, and (c) January 2004. Although other intervention points might have been selected, it was felt that this combination of points best reflected the longitudinal nature of the intervention efforts. All three intervention points were entered into each ARIMA model to determine whether a unique effect was associated with each intervention point. For example, the January 2003 intervention date model uses 1996 to 2002 pedestrian crash rates as the pre-intervention data, and 2003 to 2004 crash rates as the post-intervention data. Note that this "moving" intervention point makes it less likely that a positive effect will be shown at later points in the project (e.g., January 2004), if an effect has already been registered at some earlier point (e.g., January 2003); in other words, the downward trend would already have been incorporated into the pre-intervention data. At the same time, this approach makes it possible to determine whether a long-term intervention program has an immediate effect, or builds over time.

Although all interventions were modeled as both sudden-permanent and gradual-permanent intervention types, only the sudden-permanent models are reported because delta parameters never converged in the gradual models (Liu, 2006; McCleary \& Hay, 1980; Yaffee, 2000). The control series of pedestrian crashes in Broward County, the six metropolitan counties, and statewide excluding Miami-Dade County were entered as covariate series in separate ARIMA models to account for the preexisting downward trend in pedestrian crashes and remove other variation not related to the interventions. In some cases, when a control series (particularly Broward County) experienced an increase in pedestrian crashes during the intervention period, it would result in a significant effect that might not otherwise have been found. These are discussed in detail when they occur.

The unadjusted, average monthly pre-post changes in the pedestrian crash rates of Miami-Dade County and each control jurisdiction series for the January 2002, January 2003, and January 2004 intervention dates are shown in Table 7. These values represent the difference in the average monthly pedestrian crash rate of each series in the post-intervention date period relative to that for the average monthly pedestrian crash rate of the pre-intervention date. Hence, the effects shown are not independent (or additive) changes associated with successive dates.

Table 7. Unadjusted Post-Intervention Percentage Difference in Overall Average Monthly Pedestrian Crash Rates (per 100,000 Population) in Each Florida Jurisdiction by Intervention Date, 1996-2004.

\begin{tabular}{|l|c|c|c|}
\hline \multicolumn{1}{|c|}{ Jurisdiction } & January 2002 & January 2003 & January 2004 \\
\hline Miami-Dade County & -14.3 & -17.3 & -17.3 \\
\hline Broward County $^{\text {Six Metropolitan Counties }}{ }^{\text {a }}$ & -9.2 & -8.4 & -13.8 \\
\hline Statewide & -14.8 & -14.4 & -14.1 \\
\hline
\end{tabular}


Miami-Dade and the three comparison groups all experienced a reduction in their pedestrian crash rate during the time that the pedestrian safety program was being implemented in MiamiDade County. However, the post-intervention percentage reduction in Miami-Dade County for the latter intervention dates does appear to have been somewhat greater than that in the control jurisdictions. The reductions in the three comparison groups also varied from each other. The conclusions that can be reached from this crude description of changes in the pedestrian crash rates are that the control series used to adjust for preexisting trend in the ARIMA analyses will make a difference in the estimated effect of the pedestrian safety program in Miami-Dade County, as will the data used to estimate the impact of the interventions.

The ARIMA models assessing the overall impact of the pedestrian safety interventions in Miami-Dade County are summarized in Table 8 by the control series used to model preexisting trend and historical effects. The final models required a seasonal moving average (MA) parameter, along with the control series, to model the pre-intervention seasonality and trend in overall Miami-Dade County pedestrian crashes. Note that the two-tailed test was used for all analyses instead of the one-tailed test. This is because the two-tailed test is more stringent than the one-tailed test. Also, all of the statistical analyses conducted in the study were conducted with the Statistical Package for Social Sciences (SPSS) software, which uses two-tailed tests for all analyses.

Table 8. Summary of Sudden-Permanent ARIMA Models of Effect in Miami-Dade County for Overall Pedestrian Crash Rates per 100,000 Population Using Three Different Control

Series.

\begin{tabular}{|c|c|c|c|c|c|}
\hline Intervention & Model component & Parameter & Lag & Estimate & t-value \\
\hline \multicolumn{6}{|c|}{ Broward County Control Series } \\
\hline January 2002 & Intervention & $\omega$ & 0 & -0.2868 & -1.36 \\
\hline January 2003 & Intervention & $\omega$ & 0 & -0.8317 & $-3.43 *$ \\
\hline \multirow[t]{4}{*}{ January 2004} & Intervention & $\omega$ & 0 & 0.1149 & 0.46 \\
\hline & Broward Control series & $\beta$ & 0 & 0.5580 & $7.04 *$ \\
\hline & Noise & MA & 12 & -0.2388 & $-2.30^{*}$ \\
\hline & Constant & & & 4.2820 & $9.25 *$ \\
\hline \multicolumn{6}{|c|}{ Six Metropolitan Counties Control Series ${ }^{\mathrm{a}}$} \\
\hline January 2002 & Intervention & $\omega$ & 0 & -0.0416 & -0.20 \\
\hline January 2003 & Intervention & $\omega$ & 0 & -0.5484 & $-2.42 *$ \\
\hline \multirow[t]{4}{*}{ January 2004} & Intervention & $\omega$ & 0 & -0.1482 & -0.64 \\
\hline & Six Metropolitan Control series & $\beta$ & 0 & 0.8233 & $7.76^{*}$ \\
\hline & Noise & MA & 12 & -0.3088 & $-3.12 *$ \\
\hline & Constant & & & 3.0640 & $5.32 *$ \\
\hline \multicolumn{6}{|c|}{ Statewide (Except Miami-Dade) Control Series } \\
\hline January 2002 & Intervention & $\omega$ & 0 & -0.0529 & -0.26 \\
\hline January 2003 & Intervention & $\omega$ & 0 & -0.4956 & $-2.22 *$ \\
\hline \multirow[t]{4}{*}{ January 2004} & Intervention & $\omega$ & 0 & -0.2315 & -1.03 \\
\hline & Statewide Control series & $\beta$ & 0 & 1.1225 & $8.33 *$ \\
\hline & Noise & MA & 12 & -0.3044 & $-3.02 *$ \\
\hline & Constant & & & 2.5588 & $4.29 *$ \\
\hline
\end{tabular}


The ARIMA models across the different control series were very consistent. The only significant intervention effect found in adjusted Miami-Dade County pedestrian crashes was at the January 2003 intervention point $(p<.05)$. That is, no detectable changes in pedestrian crashes were found at the January 2002 or 2004 intervention points that were not adequately modeled using the 2003 intervention date $(p>.05)$. Rather than estimate the overall impact from models that include the other non-significant intervention parameters, the models were re-run including only the January 2003 intervention date. The resulting intervention parameters and associated effects sizes for January 2003 are shown in Table 9.

Table 9. Re-Estimated Intervention Effect Sizes for the January 2003 Intervention Point on Overall Miami-Dade Pedestrian Crashes per 100,000 Population Using Three Different Control Series.

\begin{tabular}{|l|c|c|c|}
\hline \multicolumn{1}{|c|}{ Control series } & w Estimate & \% Change & 95\% CI \\
\hline Broward County & -0.9826 & -13.3 & $-8.6,-17.9$ \\
\hline Six Metropolitan Counties ${ }^{\text {a }}$ & -0.6325 & -8.5 & $-3.7,-13.3$ \\
\hline Statewide & -0.6281 & -8.5 & $-3.8,-13.1$ \\
\hline $\begin{array}{l}\text { Note: } 95 \% \text { CI }=95 \% \text { confidence interval for the percentage change estimate. Percentage change estimates are } \\
\text { based on the average monthly January 1994-December 2002 pedestrian crash rate of 7.4 per 100,000 population. } \\
\text { a Duval, Hillsborough, Pinellas, Palm Beach, Orange, and Broward counties. }\end{array}$ \\
\hline
\end{tabular}

The total effect of the Miami-Dade pedestrian safety program was estimated to be a $13.3 \%$ reduction in pedestrian crashes based on using Broward County as a control series, and 8.5\% reduction based on using the six metropolitan counties or the statewide pedestrian crash rates as control series. Recall that Broward County had a temporary increase in its pedestrian crash rate around January 2003, which likely explains why the effect estimate is larger using this county as a control series. However, it is not clear which comparison series is the most appropriate. If Miami-Dade had still experienced this bump in pedestrian crashes in January 2003 if the pedestrian safety program had not been implemented, then Broward County would be the appropriate control. Since it did not, then the six metropolitan county or statewide series were more appropriate controls. Based on the more conservative effect estimate for these latter two control series, it appears that the Miami-Dade pedestrian safety program was associated with a reduction of 0.6 monthly pedestrian crashes per 100,000 population starting January 2003. Based on the average post-intervention monthly population in Miami-Dade County, this effect translates into a reduction of about 15 fewer pedestrian crashes per month, or about 180 fewer pedestrian crashes annually.

The 180 crashes was based on using the statewide (without Miami-Dade) control group (one of the $-8.5 \%$ estimates). The rationale was that this control group provided the most stable estimate of the expected change in Miami-Dade if the program had not been implemented. This value was selected of the three possibilities since (a) the Metropolitan County analysis would have provided the same estimate, and (b) one would have had to argue that the pedestrian crash rate would have increased in Miami-Dade during the post-intervention period like it seems to have done in Broward County in order to choose that analysis as the better counterfactual representation of Miami-Dade. 
The effect estimate from the statewide control series analysis provides an estimate of 0.6281 fewer monthly pedestrian crashes per 100,000 population for Miami-Dade. The average monthly population of Miami-Dade in the post-2003 period $(2,383,733)$ was then divided by 100,000 to get the monthly average number of hundreds of thousands of population in Miami-Dade (23.84). This figure was multiplied by the 0.6281 to get the monthly estimate of crashes saved (14.97), and finally multiplied by 12 to get an annual estimate of 179.67 , or approximately 180 crashes per year.

If Broward had been chosen as the appropriate control for calculating this figure, it would have been 281 crashes per year. To justify this figure would have required making the argument that pedestrian crashes in Miami-Dade would have increased in the post-intervention period like they did in Broward, which was not considered to be defendable given that the other two control groups gave such a consistent answer.

It should also be mentioned that the benefits of the pedestrian safety program continued beyond 2003 in that the average number of pedestrian crashes in 2004 remained lower than the pre-2003 level. However, there was no independent additive reduction detected that could be associated with the pedestrian safety activities conducted during 2004. The ARIMA analyses showed that there was a large reduction in pedestrian crashes in Miami-Dade County during the combined 2003 to 2004 time period after adjusting for other temporal trends (e.g., fuel prices and changes in traffic safety laws) and seasonality using the various comparison series of Florida jurisdictions. The conclusion that this reduction can largely be attributed to the overall pedestrian safety program is supported by the fact that the reductions in Miami-Dade pedestrian crashes were consistently larger than those for other Florida jurisdictions, regardless of how the comparison group was formed.

In summary, pedestrian crashes in Miami-Dade County were reduced by 180 per year for a total of 360 fewer pedestrian crashes during the two year 2003-2004 "after" period. The most likely cause of this reduction is the combined pedestrian safety program efforts that began in 2003. The fact that pedestrian crashes per month leveled off during 2004 may indicate that additional countermeasures (or increased countermeasure intensity) are needed to achieve additional reductions in the monthly rate of pedestrian crashes after 2004, or that additional data points are necessary to be able to detect any additional independent effect of the activities in 2004.

\section{Program Impact by Pedestrian Age}

The Miami-Dade crashes were categorized according to the age of the pedestrian and analyzed separately to determine if the safety program had a differential impact on pedestrians across age groups. The age groups into which the crashes were categorized were: (a) 1 to 13, (b) 14 to 64, and (c) 65 or older. Multivariate ARIMA intervention analyses of per 100,000 population crash rates were again conducted using the corresponding group from the control jurisdictions to remove trend and historical variation. The results are presented in the following three subsections of the report. 


\section{Ages 1 to 13}

The pedestrian crash rates for 1- to 13-year-olds are shown in Figure 52, and the raw pre-post percentage differences for each intervention point are shown in Table 10.

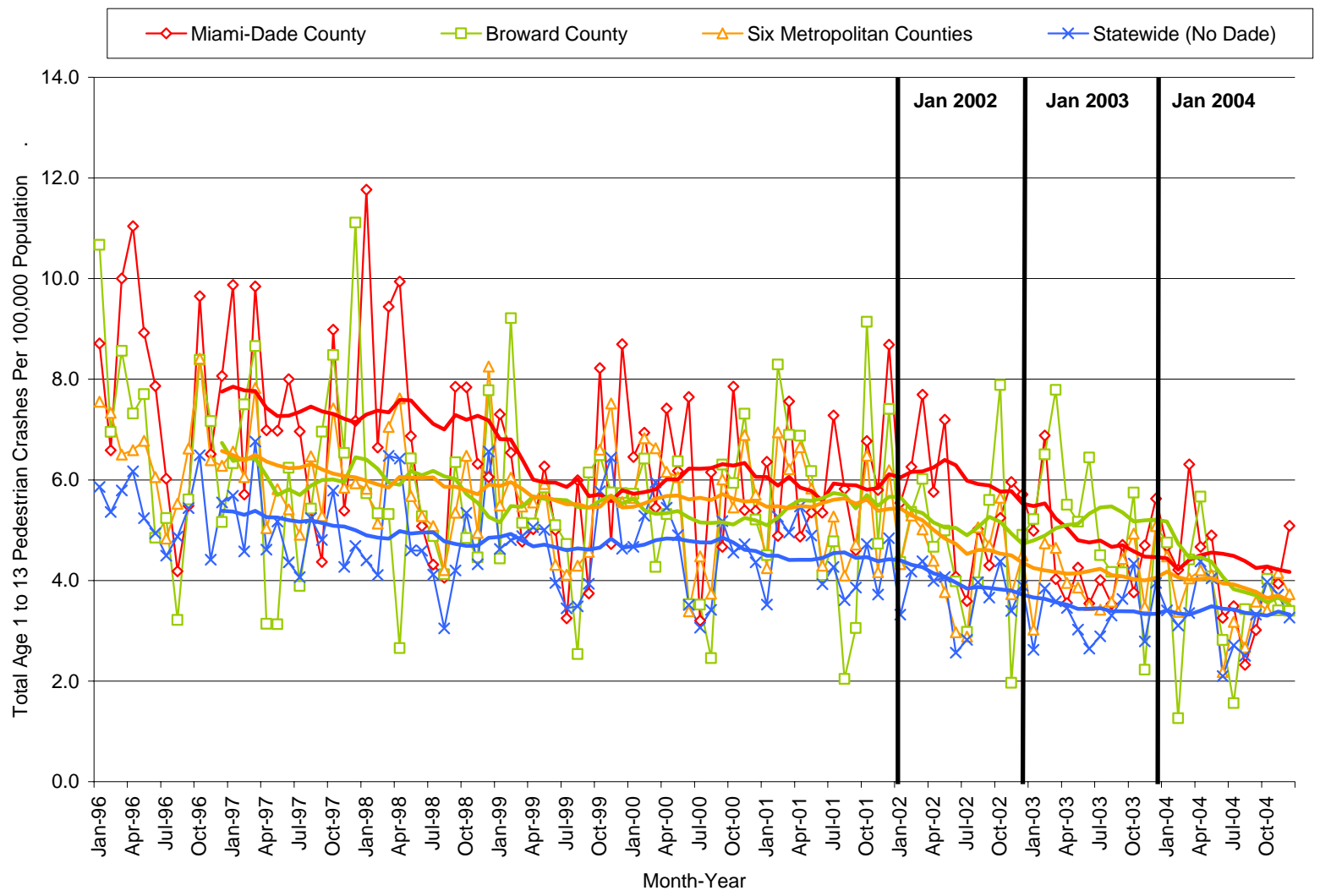

Figure 52. Monthly 1- to 13-Year-Olds' Pedestrian Crashes per 100,000 Population in Miami-Dade County, Broward County, Six Metropolitan Counties (Combined), and Statewide From 1996-2004.

Table 10. Unadjusted Post-Intervention Percentage Difference in 1- to 13-Year-Olds' Average Monthly Pedestrian Crash Rates in Each Florida Jurisdiction by Intervention Date, 1996-2004.

\begin{tabular}{|c|c|c|c|}
\hline Jurisdiction & January 2002 & January 2003 & January 2004 \\
\hline Miami-Dade County & -29.3 & -33.7 & -33.3 \\
\hline Broward County & -22.8 & -22.8 & -37.1 \\
\hline Six Metropolitan Counties $^{\mathrm{a}}$ & -31.4 & -32.0 & -33.8 \\
\hline Statewide & -28.2 & -28.5 & -26.1 \\
\hline
\end{tabular}


Although Miami-Dade experienced a large decrease in pedestrian crashes among 1- to 13-yearolds, so did all the control jurisdictions. The decrease was initially somewhat smaller for Broward County, which was likely due to the increase, or bump, that occurred during 2003. The results of the ARIMA analyses are summarized in Table 11.

Table 11. Summary of Sudden-Permanent ARIMA Models of Effect in Miami-Dade County for 1- to 13-Year-Olds’ Pedestrian Crash Rates per 100,000 Population Using Three Different Control Series.

\begin{tabular}{|c|c|c|c|c|c|}
\hline Intervention & Model component & Parameter & Lag & Estimate & t-value \\
\hline \multicolumn{6}{|c|}{ Broward County Control Series } \\
\hline January 2002 & Intervention & $\omega$ & 0 & -0.6296 & -1.35 \\
\hline January 2003 & Intervention & $\omega$ & 0 & -1.2069 & $-2.01 *$ \\
\hline \multirow[t]{3}{*}{ January 2004} & Intervention & $\omega$ & 0 & 0.2149 & 0.35 \\
\hline & Broward Control series & $\beta$ & 0 & 0.3012 & $3.63 *$ \\
\hline & Constant & & & 4.7332 & $9.25 *$ \\
\hline \multicolumn{6}{|c|}{ Six Metropolitan Counties Control Series ${ }^{a}$} \\
\hline January 2002 & Intervention & $\omega$ & 0 & 0.0202 & 0.04 \\
\hline January 2003 & Intervention & $\omega$ & 0 & -0.8362 & -1.49 \\
\hline \multirow[t]{3}{*}{ January 2004} & Intervention & $\omega$ & 0 & 0.0493 & 0.09 \\
\hline & Six Metropolitan Control series & $\beta$ & 0 & 0.7427 & $5.49 *$ \\
\hline & Constant & & & 2.2708 & $2.82 *$ \\
\hline \multicolumn{6}{|c|}{ Statewide (Except Miami-Dade) Control Series } \\
\hline January 2002 & Intervention & $\omega$ & 0 & 0.0946 & 0.21 \\
\hline January 2003 & Intervention & $\omega$ & 0 & -0.6579 & -1.22 \\
\hline \multirow[t]{3}{*}{ January 2004} & Intervention & $\omega$ & 0 & -0.2765 & -0.51 \\
\hline & Statewide Control series & $\beta$ & 0 & 1.0579 & $6.52 *$ \\
\hline & Constant & & & 1.4975 & $1.98^{*}$ \\
\hline
\end{tabular}

The results of the multivariate ARIMA analyses did not indicate a significant change in the pedestrian crash rates of 1- to 13-year-olds at any of the intervention dates using the six metropolitan county or statewide control series $(p>.05)$. The results did indicate a significant reduction in 1- to 13-year-olds' pedestrian crashes as of January 2003 using the Broward County control series, which is probably due to the increase in 1- to 13-year-olds' pedestrian crashes that Broward County experienced in 2003, which did not occur in neighboring Miami-Dade County. If one can argue that a similar increase would have occurred in Miami-Dade County, had the pedestrian safety interventions not been implemented, then this observed decrease would be associated with 1.2 fewer monthly pedestrian crashes per 100,000 1- to 13-year-olds in MiamiDade County, which is about an $18.5 \%$ decrease.

\section{Age 5 to 12}

Because a large number of the countermeasures were specifically aimed at young children, the analyses were repeated using only pedestrian crashes among children 5 to 12 years old. The pedestrian crash rates for 5- to 12-year-olds are shown in Figure 53, and the raw pre-post percentage differences for each intervention point are shown in Table 12. 


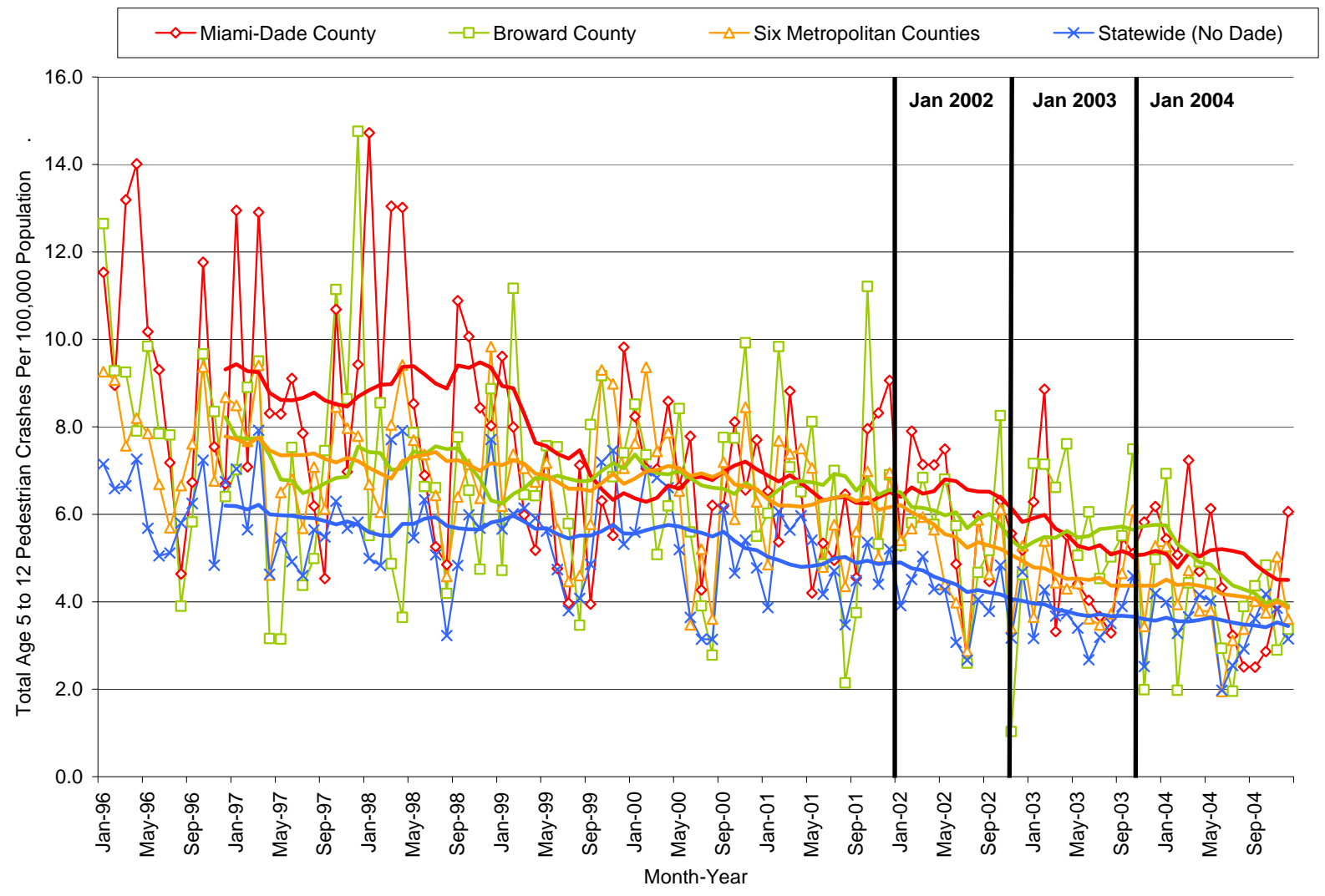

\section{Figure 53. Monthly 5- to 12-Year-Olds’ Pedestrian Crashes per 100,000 Population in Miami-Dade County, Broward County, Six Metropolitan Counties (Combined), and Statewide From 1996-2004.}

The descriptive data were consistent with the prior results for all children under age 13 in that all of the jurisdictions experienced a large decrease in child pedestrian crashes during the study time period. The decrease for Broward County was again initially not as large as was the case for the other jurisdictions. The results of the multivariate ARIMA analyses (Table 13) did not indicate a change in the pedestrian crash rates of 5- to 12-year-olds in Miami-Dade County that was significantly different from those in any of the other jurisdictions at any of the intervention dates $(p>.05)$, even though unadjusted crash reductions resulted in the 5- to 12-year-old children in Miami-Dade County during each of the intervention periods. The lack of a significant effect (after adjusting for the control groups) is likely the result of the decreasing trend in child crashes in the control groups plus limitations in the sample of crashes. 
Table 12. Unadjusted Post-Intervention Percentage Difference in 5- to 12-Year-Olds' Average Monthly Pedestrian Crash Rates in Each Florida Jurisdiction by Intervention

Date, 1996-2004.

\begin{tabular}{|l|c|c|c|}
\hline \multicolumn{1}{|c|}{ Jurisdiction } & January 2002 & January 2003 & January 2004 \\
\hline Miami-Dade County & -34.6 & -36.4 & -38.3 \\
\hline Broward County & -29.6 & -28.7 & -41.3 \\
\hline Six Metropolitan Counties ${ }^{\text {a }}$ & -36.7 & -38.1 & -39.3 \\
\hline Statewide & -33.9 & -34.4 & -32.8 \\
\hline $\begin{array}{l}\text { Note. Each percentage difference represents the difference in the average monthly pedestrian crash rate per } \\
\text { 100,000 population in the post-intervention period relative to the pre-intervention average monthly rate. } \\
\text { a Duval, Hillsborough, Pinellas, Palm Beach, Orange, and Broward counties. }\end{array}$ \\
\hline
\end{tabular}

Table 13. Summary of Sudden-Permanent ARIMA Models for 5- to 12-Year-Olds' Pedestrian Crash Rates per 100,000 Population Using Three Different Control Series.

\begin{tabular}{|c|c|c|c|c|c|}
\hline Intervention & Model component & Parameter & Lag & Estimate & t-value \\
\hline \multicolumn{6}{|c|}{ Broward County Control Series } \\
\hline January 2002 & Intervention & $\omega$ & 0 & -1.2172 & -1.87 \\
\hline January 2003 & Intervention & $\omega$ & 0 & -0.8242 & -1.00 \\
\hline \multirow[t]{3}{*}{ January 2004} & Intervention & $\omega$ & 0 & -0.1140 & -0.14 \\
\hline & Control series & $\beta$ & 0 & 0.2970 & $3.27^{*}$ \\
\hline & Constant & & & 5.4932 & $8.05^{*}$ \\
\hline \multicolumn{6}{|c|}{ Six Metropolitan Counties Control Series ${ }^{\mathrm{a}}$} \\
\hline January 2002 & Intervention & $\omega$ & 0 & -0.3170 & -0.49 \\
\hline January 2003 & Intervention & $\omega$ & 0 & -0.2469 & -0.32 \\
\hline \multirow[t]{3}{*}{ January 2004} & Intervention & $\omega$ & 0 & -0.2872 & -0.37 \\
\hline & Control series & $\beta$ & 0 & 0.7384 & $5.32 *$ \\
\hline & Constant & & & 2.4982 & $2.53 *$ \\
\hline \multicolumn{6}{|c|}{ Statewide (Except Miami-Dade) Control Series } \\
\hline January 2002 & Intervention & $\omega$ & 0 & -0.2398 & -0.38 \\
\hline January 2003 & Intervention & $\omega$ & 0 & -0.1997 & -0.26 \\
\hline \multirow[t]{3}{*}{ January 2004} & Intervention & $\omega$ & 0 & -0.5430 & -0.72 \\
\hline & Control series & $\beta$ & 0 & 1.0098 & $5.73^{*}$ \\
\hline & Constant & & & 2.0020 & $1.99^{*}$ \\
\hline
\end{tabular}




\section{Age 14 to 64}

The pedestrian crash rates for 14- to 64-year-olds are shown in Figure 54, and the raw pre-post percentage differences for each intervention point are shown in Table 14.

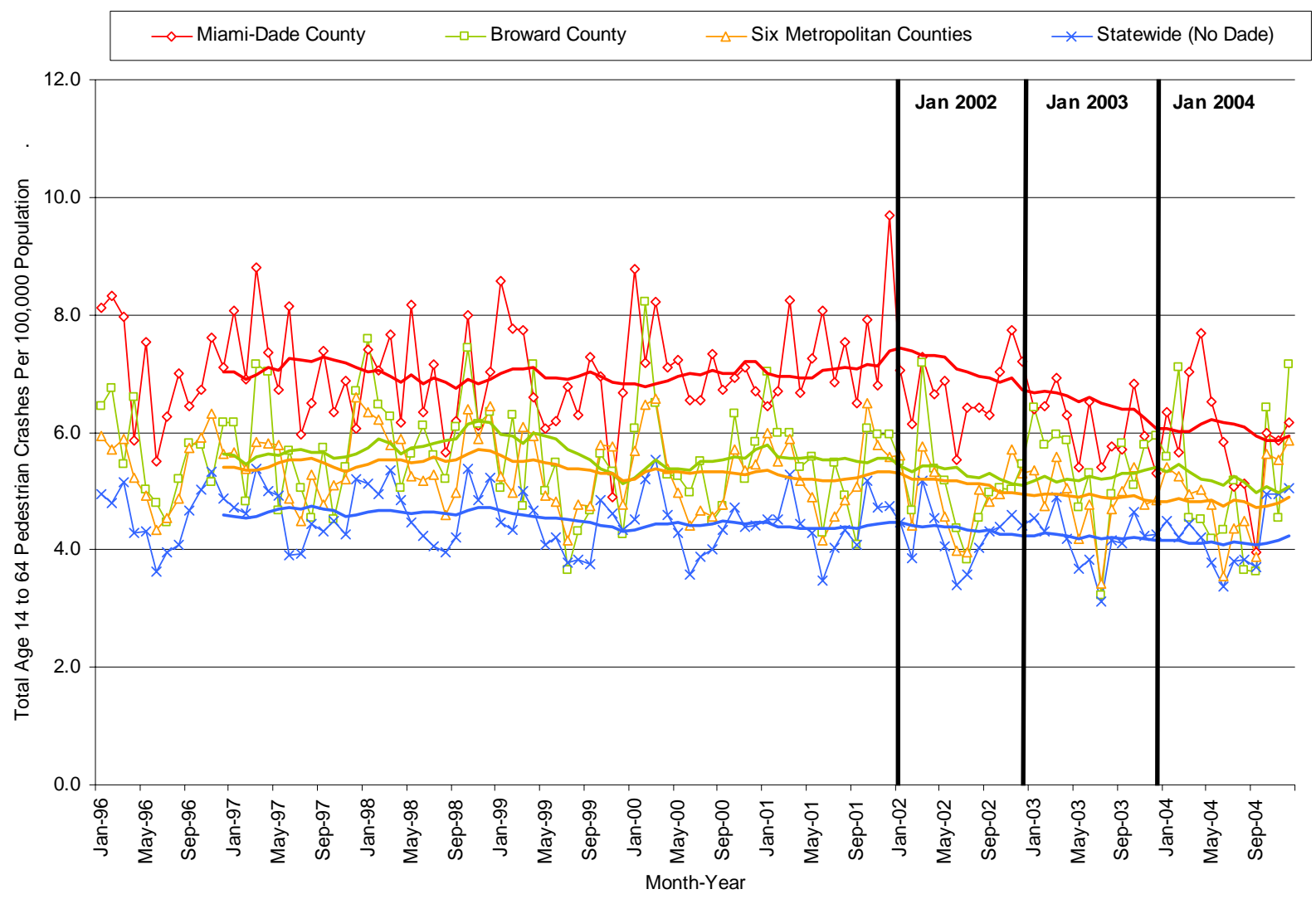

Figure 54. Monthly 14- to 64-Year-Olds' Pedestrian Crashes per 100,000 Population in Miami-Dade County, Broward County, Six Metropolitan Counties (Combined), and Statewide From 1996-2004. 
Table 14. Unadjusted Post-Intervention Percentage Difference in 14- to 64-Year-Olds' Average Monthly Pedestrian Crash Rates in Each Florida Jurisdiction by Intervention Date, 1996-2004.

\begin{tabular}{|c|c|c|c|}
\hline Jurisdiction & January 2002 & January 2003 & January 2004 \\
\hline Miami-Dade County & -11.7 & -14.5 & -14.0 \\
\hline Broward County & -7.9 & -5.8 & -8.4 \\
\hline Six Metropolitan Counties ${ }^{a}$ & -9.3 & -8.9 & -7.1 \\
\hline Statewide & -6.9 & -6.3 & -4.8 \\
\hline
\end{tabular}

Although all the Florida jurisdictions analyzed experienced reductions in 14- to 64-year-olds' pedestrian crashes during the time that the pedestrian safety project was implemented, the reductions for Miami-Dade appear to be larger in magnitude. The results of the ARIMA analyses are shown in Table 15.

The ARIMA analyses of 14- to 64-year-olds' pedestrian crash rates indicate a significant reduction in crashes among this age group in Miami-Dade County starting January 2003, regardless of the control series used to remove the downward trend $(p<.05)$. No changes were indicated at the January 2002 or January 2004 intervention dates $(p>.05)$. Using the statewide control series to estimate the magnitude of this effect, the 2003 intervention date was associated with a 0.60 monthly reduction in Miami-Dade 14- to 64-year-olds' pedestrian crashes per 100,000 population, or about an $8.6 \%$ reduction from the average prior level. 
Table 15. Summary of Sudden-Permanent ARIMA Models of Effect in Miami-Dade County for 14- to 64-Year-Olds' Pedestrian Crash Rates per 100,000 Population Using Three Different Control Series.

\begin{tabular}{|c|c|c|c|c|c|}
\hline Intervention & Model component & Parameter & Lag & Estimate & t-value \\
\hline \multicolumn{6}{|c|}{ Broward County Control Series } \\
\hline January 2002 & Intervention & $\omega$ & 0 & -0.1914 & -0.81 \\
\hline January 2003 & Intervention & $\omega$ & 0 & -0.7175 & $-2.36^{*}$ \\
\hline \multirow[t]{3}{*}{ January 2004} & Intervention & $\omega$ & 0 & -0.0669 & -0.22 \\
\hline & Broward Control series & $\beta$ & 0 & 0.2285 & $2.92 *$ \\
\hline & Constant & & & 5.7497 & $12.77 *$ \\
\hline \multicolumn{6}{|c|}{ Six Metropolitan Counties Control Series ${ }^{\text {a }}$} \\
\hline January 2002 & Intervention & $\omega$ & 0 & -0.1412 & -0.65 \\
\hline January 2003 & Intervention & $\omega$ & 0 & -0.5824 & $-2.09 *$ \\
\hline \multirow[t]{3}{*}{ January 2004} & Intervention & $\omega$ & 0 & -0.1774 & -0.64 \\
\hline & Six Metropolitan Control series & $\beta$ & 0 & 0.4760 & $4.49 *$ \\
\hline & Constant & & & 4.5056 & $7.81 *$ \\
\hline \multicolumn{6}{|c|}{ Statewide (Except Miami-Dade) Control Series } \\
\hline January 2002 & Intervention & $\omega$ & 0 & -0.1538 & -0.73 \\
\hline January 2003 & Intervention & $\omega$ & 0 & -0.6033 & $-2.21 *$ \\
\hline \multirow[t]{3}{*}{ January 2004} & Intervention & $\omega$ & 0 & -0.1848 & -0.68 \\
\hline & Statewide Control series & $\beta$ & 0 & 0.6678 & $5.05^{*}$ \\
\hline & Constant & & & 4.0475 & $6.70 *$ \\
\hline
\end{tabular}

\section{Age 65 and Older}

The crash rates for 65-and-older pedestrians are shown in Figure 55, and the raw pre-post percentage differences for each intervention point are shown in Table 16.

Again, the average crash rate was lower among all the Florida jurisdictions, but in most cases there appeared to be less of a reduction in Miami-Dade County than in the other jurisdictions. The results of the ARIMA analyses are summarized in Table 17. 


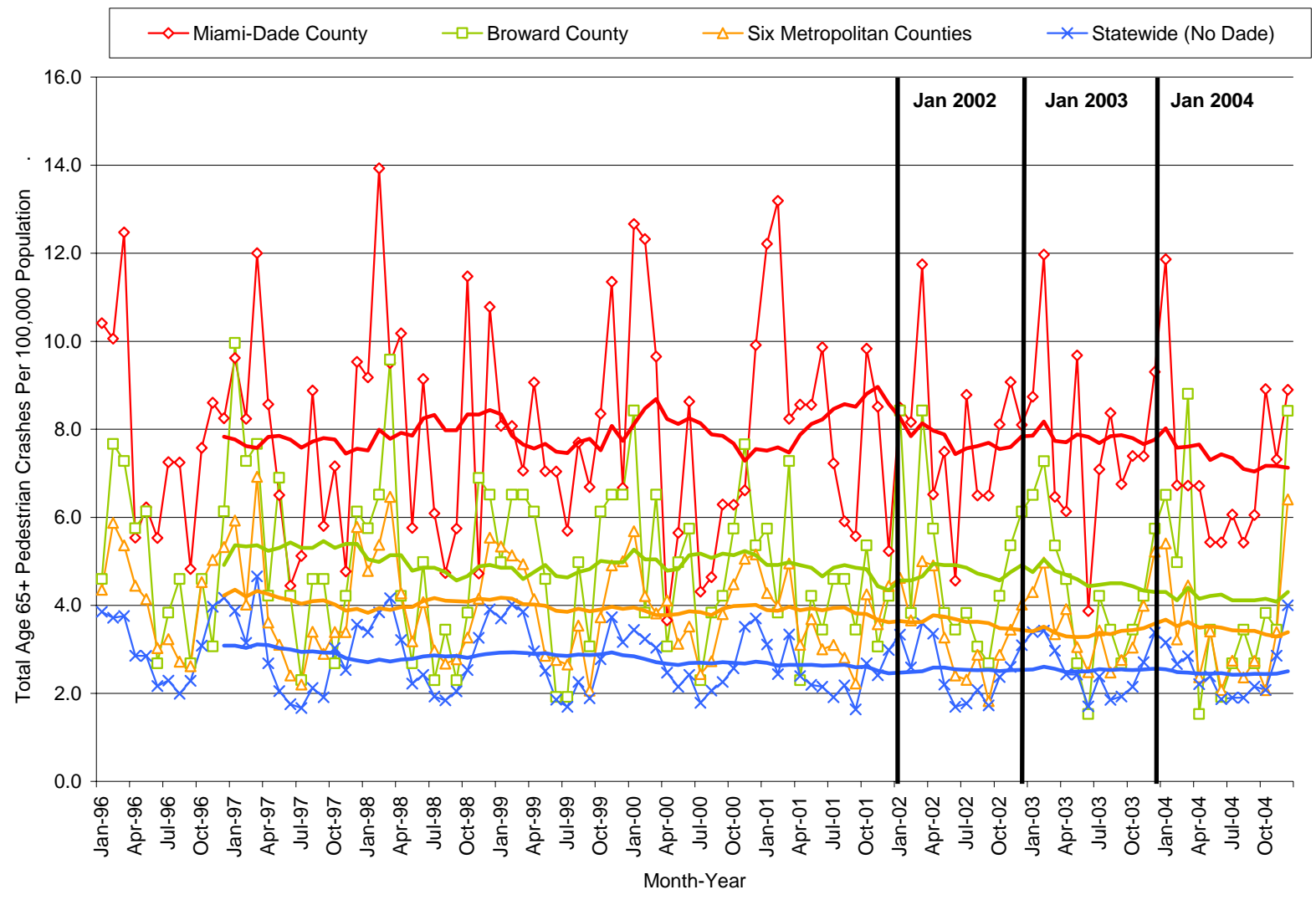

Figure 55. Monthly 65-and-Older Pedestrian Crashes per 100,000 Population in MiamiDade County, Broward County, Six Metropolitan Counties (Combined), and Statewide From 1996-2004.

\section{Table 16. Unadjusted Post-Intervention Percentage Difference in 65+ Age Group Average Monthly Pedestrian Crash Rates in Each Florida Jurisdiction by Intervention Date, 1996-2004.}

\begin{tabular}{|c|c|c|c|}
\hline Jurisdiction & January 2002 & January 2003 & January 2004 \\
\hline Miami-Dade County & -4.7 & -6.1 & -9.9 \\
\hline Broward County & -8.9 & -12.9 & -11.5 \\
\hline Six Metropolitan Counties ${ }^{a}$ & -12.6 & -10.5 & -12.1 \\
\hline Statewide & -9.4 & -8.2 & -8.5 \\
\hline
\end{tabular}




\section{Table 17. Summary of Sudden-Permanent ARIMA Models of Effect in Miami-Dade County for 65-and-Older Pedestrian Crash Rates per 100,000 Population Using Three Different Control Series.}

\begin{tabular}{|c|c|c|c|c|c|}
\hline Intervention & Model component & Parameter & Lag & Estimate & t-value \\
\hline \multicolumn{6}{|c|}{ Broward County Control Series } \\
\hline January 2002 & Intervention & $\omega$ & 0 & -0.0631 & -0.12 \\
\hline January 2003 & Intervention & $\omega$ & 0 & 0.2454 & 0.33 \\
\hline \multirow[t]{4}{*}{ January 2004} & Intervention & $\omega$ & 0 & -0.6844 & -0.91 \\
\hline & Noise & MA & 7 & 0.2610 & $2.74 *$ \\
\hline & Broward Control series & $\beta$ & 0 & 0.5276 & $5.12 *$ \\
\hline & Constant & & & 5.3329 & $9.91 *$ \\
\hline \multicolumn{6}{|c|}{ Six Metropolitan Counties Control Series ${ }^{\text {a }}$} \\
\hline January 2002 & Intervention & $\omega$ & 0 & 0.6749 & 1.33 \\
\hline January 2003 & Intervention & $\omega$ & 0 & -0.1509 & -0.22 \\
\hline \multirow[t]{4}{*}{ January 2004} & Intervention & $\omega$ & 0 & -0.4912 & -0.70 \\
\hline & Noise & MA & 7 & 0.2146 & $2.13 *$ \\
\hline & Six Metropolitan Control series & $\beta$ & 0 & 1.1616 & $6.89 *$ \\
\hline & Constant & & & 3.1326 & $4.54^{*}$ \\
\hline \multicolumn{6}{|c|}{ Statewide (Except Miami-Dade) Control Series } \\
\hline January 2002 & Intervention & $\omega$ & 0 & 0.5730 & 1.16 \\
\hline January 2003 & Intervention & $\omega$ & 0 & -0.0507 & -0.07 \\
\hline \multirow[t]{4}{*}{ January 2004} & Intervention & $\omega$ & 0 & -0.5791 & -0.84 \\
\hline & Noise & MA & 7 & 0.2192 & $2.20 *$ \\
\hline & Statewide Control series & $\beta$ & 0 & 1.8715 & $7.33 *$ \\
\hline & Constant & & & 2.4955 & $3.41 *$ \\
\hline
\end{tabular}

None of the ARIMA models indicated a significant change in 65-and-older pedestrian crash rates in Miami-Dade County at any of the intervention points $(p>.05)$ after controlling for variability using the control series. This finding is consistent with the graph, which does not show a consistent change subsequent to implementing the pedestrian safety program.

\section{Program Impact by Pedestrian Gender}

To determine if the pedestrian safety program had a different impact on males and females, the Miami-Dade pedestrian crashes were categorized according to the gender of the involved pedestrian and analyzed again using multivariate ARIMA intervention models.

\section{$\underline{\text { Crashes Among Males }}$}

The pedestrian crash rates for males are shown in Figure 56, and the raw pre-post percentage differences for each intervention point are shown in Table 18. 


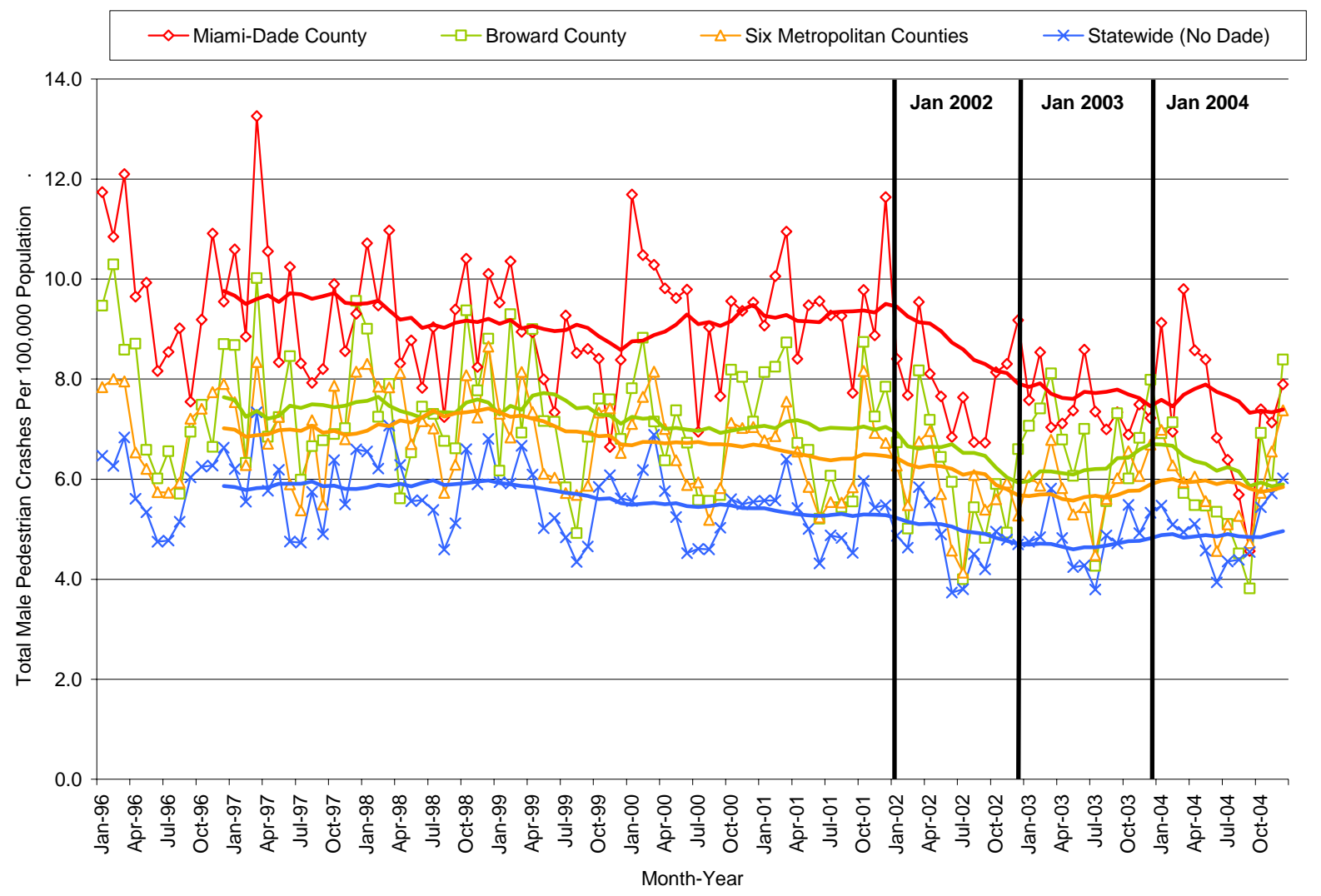

Figure 56. Monthly Male Pedestrian Crashes per 100,000 Population in Miami-Dade County, Broward County, Six Metropolitan Counties (Combined), and Statewide from 1996-2004.

Table 18. Unadjusted Post-Intervention Percentage Difference in Male Average Monthly Pedestrian Crash Rates in Each Florida Jurisdiction by Intervention Date, 1996-2004.

\begin{tabular}{|c|c|c|c|}
\hline Jurisdiction & January 2002 & January 2003 & January 2004 \\
\hline Miami-Dade County & -18.8 & -18.7 & -17.2 \\
\hline Broward County & -15.6 & -11.6 & -16.6 \\
\hline Six Metropolitan Counties ${ }^{\mathrm{a}}$ & -15.4 & -12.4 & -11.4 \\
\hline Statewide & -14.4 & -11.2 & -8.5 \\
\hline \multicolumn{4}{|c|}{$\begin{array}{l}\text { Note. Each percentage difference represents the difference in the average monthly pedestrian crash rate per } \\
100,000 \text { population in the post-intervention period relative to the pre-intervention average monthly rate. } \\
\text { a Duval, Hillsborough, Pinellas, Palm Beach, Orange, and Broward counties. }\end{array}$} \\
\hline
\end{tabular}

Although all jurisdictions had observed reductions in male pedestrian crashes, those for MiamiDade County appear to be larger at all three time points. Note the bump in pedestrian crashes in Broward County during 2003. The ARIMA analyses are summarized in Table 19.

The results of the ARIMA analyses indicated a significant reduction in pedestrian crashes in 2002 (i.e., the effect beginning in January 2002), regardless of the control series used ( $p<.05)$. In addition, using the Broward County control series an additional reduction was found in 2003 
$(p<.05)$, but not using the other jurisdiction control series $(p>.05)$. Using the statewide control model to characterize the 2002 effect, the implementation of the pedestrian safety program was associated with a 0.7 monthly reduction in male pedestrian crashes per 100,000 population, or an average $7.3 \%$ reduction relative to the prior series level.

Interpreting the Broward County effect beginning in January 2003 involves accepting that the increase in male pedestrian crashes observed in Broward County would have happened in Miami-Dade, had the pedestrian safety project not been implemented. If this is true, then the effect beginning in January 2003 is associated with 0.9 fewer monthly crashes per 100,000 males in Miami-Dade County, or a $9.4 \%$ reduction.

\section{Table 19. Summary of Sudden-Permanent ARIMA Models of Effect in Miami-Dade County for Male Pedestrian Crash Rates per 100,000 Population Using Three Different Control Series.}

\begin{tabular}{|c|c|c|c|c|c|}
\hline Intervention & Model component & Parameter & Lag & Estimate & t-value \\
\hline \multicolumn{6}{|c|}{ Broward County Control Series } \\
\hline January 2002 & Intervention & $\omega$ & 0 & -0.6963 & $-2.13^{*}$ \\
\hline January 2003 & Intervention & $\omega$ & 0 & -0.8608 & $-2.11 *$ \\
\hline \multirow[t]{3}{*}{ January 2004} & Intervention & $\omega$ & 0 & 0.3636 & 0.89 \\
\hline & Broward Control series & $\beta$ & 0 & 0.5270 & $6.62 *$ \\
\hline & Constant & & & 5.4847 & $9.22 *$ \\
\hline \multicolumn{6}{|c|}{ Six Metropolitan Counties Control Series ${ }^{\mathrm{a}}$} \\
\hline January 2002 & Intervention & $\omega$ & 0 & -0.7168 & $-2.01 *$ \\
\hline January 2003 & Intervention & $\omega$ & 0 & -0.5833 & -1.35 \\
\hline \multirow[t]{3}{*}{ January 2004} & Intervention & $\omega$ & 0 & -0.0342 & -0.08 \\
\hline & Six Metropolitan Control series & $\beta$ & 0 & 0.5990 & $5.07 *$ \\
\hline & Constant & & & 5.2306 & $6.37 *$ \\
\hline \multicolumn{6}{|c|}{ Statewide (Except Miami-Dade) Control Series } \\
\hline January 2002 & Intervention & $\omega$ & 0 & -0.6792 & $-2.01 *$ \\
\hline January 2003 & Intervention & $\omega$ & 0 & -0.5545 & -1.36 \\
\hline \multirow[t]{3}{*}{ January 2004} & Intervention & $\omega$ & 0 & -0.1841 & -0.45 \\
\hline & Statewide Control series & $\beta$ & 0 & 0.8417 & $5.95^{*}$ \\
\hline & Constant & & & 4.6353 & $5.75^{*}$ \\
\hline
\end{tabular}

\section{Crashes Among Females}

The pedestrian crash rates for females are shown in Figure 57, and the raw pre-post percentage differences for each intervention point are shown in Table 20. 


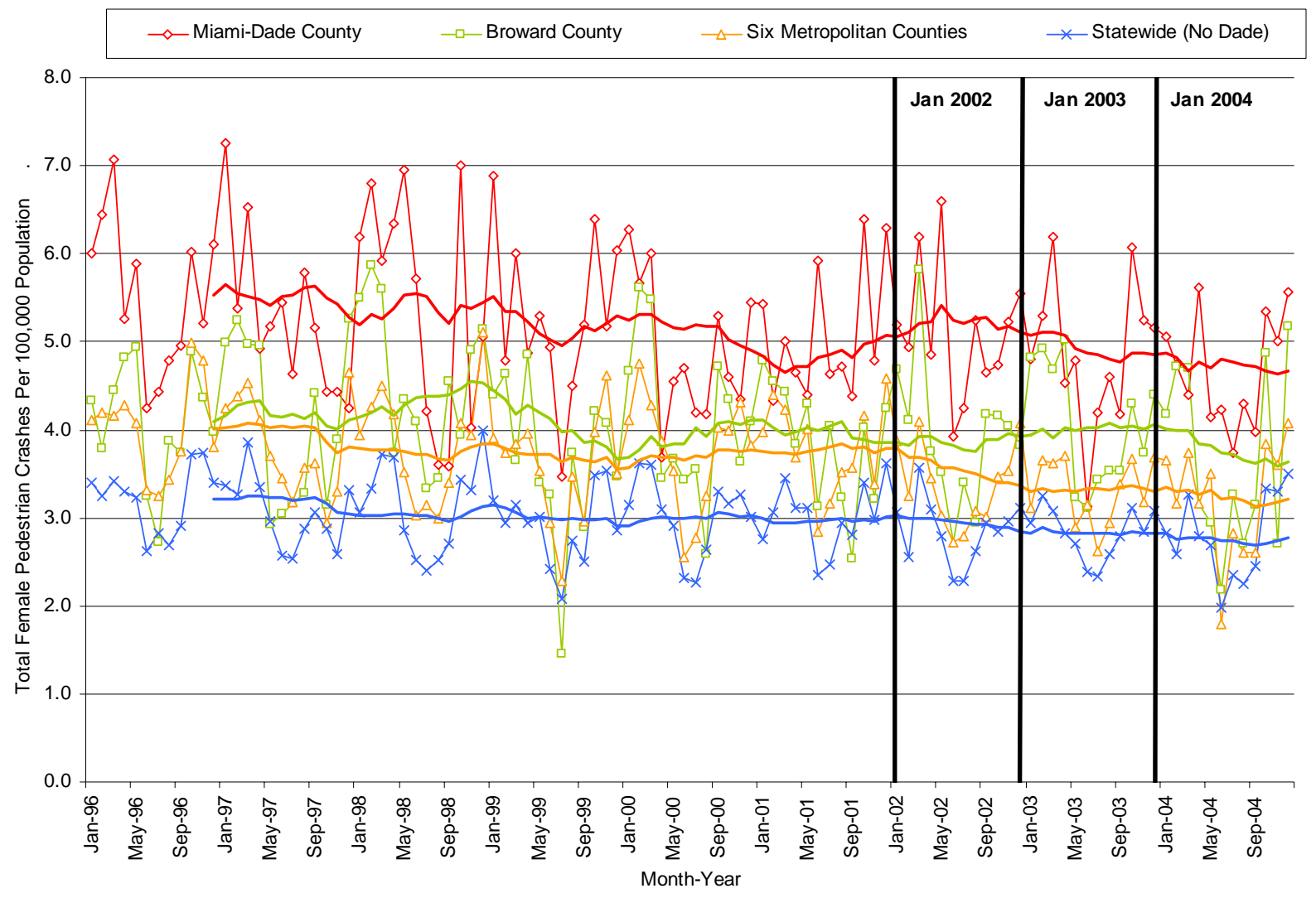

Figure 57. Monthly Female Pedestrian Crashes per 100,000 Population in Miami-Dade County, Broward County, Six Metropolitan Counties (Combined), and Statewide from 1996-2004.

Table 20. Unadjusted Post-Intervention Percentage Difference in Female Average Monthly Pedestrian Crash Rates in Each Florida Jurisdiction by Intervention Date, 1996-2004.

\begin{tabular}{|l|c|c|c|}
\hline \multicolumn{1}{|c|}{ Jurisdiction } & January 2002 & January 2003 & January 2004 \\
\hline Miami-Dade County & -7.3 & -9.1 & -9.9 \\
\hline Broward County & -4.5 & -4.7 & -9.9 \\
\hline Six Metropolitan Counties ${ }^{\text {a }}$ & -13.3 & -12.8 & -12.8 \\
\hline Statewide & -7.8 & -7.3 & -7.4 \\
\hline Note: Each percentage difference represents the difference in the average monthly pedestrian crash rate per \\
$\begin{array}{l}\text { 100,000 population in the post-intervention period relative to the pre-intervention average monthly rate. } \\
{ }^{2} \text { Duval, Hillsborough, Pinellas, Palm Beach, Orange, and Broward counties. }\end{array}$
\end{tabular}

There visually does not appear to be a consistent decrease in female pedestrian crashes in MiamiDade County during the period after the pedestrian safety project was implemented. Although on average all jurisdictions experienced a decrease in female pedestrian crashes, those for MiamiDade County are actually smaller than the reductions observed in other jurisdictions. If anything, the figure suggests that Miami-Dade County experienced an increase in female pedestrian crashes in the post time period, which trended back down after 2002, but still had not returned to 
the level expected according to the preexisting downward trend by the end of the study time period. The results of the ARIMA analyses are summarized in Table 21.

Table 21. Summary of Sudden-Permanent ARIMA Models of Effect in Miami-Dade County for Female Pedestrian Crash Rates per 100,000 Population Using Three Different Control Series.

\begin{tabular}{|c|c|c|c|c|c|}
\hline Intervention & Model component & Parameter & Lag & Estimate & t-value \\
\hline \multicolumn{6}{|c|}{ Broward County Control Series } \\
\hline January 2002 & Intervention & $\omega$ & 0 & -0.0422 & -0.21 \\
\hline January 2003 & Intervention & $\omega$ & 0 & -0.3818 & -1.39 \\
\hline \multirow[t]{4}{*}{ January 2004} & Intervention & $\omega$ & 0 & 0.0484 & 0.17 \\
\hline & Noise & MA & 4 & 0.2139 & $2.27 *$ \\
\hline & Broward Control series & $\beta$ & 0 & 0.4393 & $4.82 *$ \\
\hline & Constant & & & 3.4613 & $9.15^{*}$ \\
\hline \multicolumn{6}{|c|}{ Six Metropolitan Counties Control Series ${ }^{a}$} \\
\hline January 2002 & Intervention & $\omega$ & 0 & 0.1558 & 0.63 \\
\hline January 2003 & Intervention & $\omega$ & 0 & -0.2199 & -0.70 \\
\hline \multirow[t]{3}{*}{ January 2004} & Intervention & $\omega$ & 0 & -0.1086 & -0.35 \\
\hline & Six Metropolitan Control series & $\beta$ & 0 & 0.7022 & $5.24 *$ \\
\hline & Constant & & & 2.5920 & $5.01 *$ \\
\hline \multicolumn{6}{|c|}{ Statewide (Except Miami-Dade) Control Series } \\
\hline January 2002 & Intervention & $\omega$ & 0 & 0.0966 & 0.42 \\
\hline January 2003 & Intervention & $\omega$ & 0 & -0.2457 & -0.83 \\
\hline \multirow[t]{3}{*}{ January 2004} & Intervention & $\omega$ & 0 & -0.1149 & -0.39 \\
\hline & Statewide Control series & $\beta$ & 0 & 1.1602 & $6.77 *$ \\
\hline & Constant & & & 1.7164 & $3.24 *$ \\
\hline
\end{tabular}

The ARIMA analyses for female pedestrian crashes did not indicate a significant change in the Miami-Dade County series at any of the intervention points using any of the control series $(p>$ .05). The implementation of the pedestrian safety program in Miami-Dade County did not appear to have an overall impact on the rate of female pedestrian crashes in Miami-Dade County.

It should be mentioned that no individual treatments were directed specifically at males as part of the child pedestrian education programs. One possible explanation for the higher reduction in pedestrian crashes among males is that since males made up a majority of the pedestrian crashes in the before period, there was more potential for the crash reduction among the male population.

\section{Program Impact by Time of Day}

One would expect different types of pedestrians to be involved in crashes occurring during different parts of the day. In particular, it would be expected that schoolchildren would be more likely to be involved in pedestrian crashes during the times when they would be walking to school during the morning hours (6 a.m. to 9:59 a.m.) or when they are out of school at the end of the school day (2 p.m. to 5:59 p.m.). To determine if the pedestrian safety program had an impact on pedestrian crashes during different times of the day, the total crashes were categorized 
into one of the following five time periods: (a) 6 a.m. to 9:59 a.m., (b) 10 a.m. to 1:59 p.m., (c) 2 p.m. to 5:59 p.m. , (d) 6 p.m. to 9:59 p.m. , or (e) 10 p.m. to 5:59 a.m.. Crashes during each time period were analyzed again using multivariate ARIMA intervention analyses, presented in the following subsections.

Crashes from 6 a.m. to 9:59 a.m.

The pedestrian crash rates during 6 a.m. to 9:59 a.m. are shown in Figure 58, and the raw prepost percentage differences for each intervention point are shown in Table 22. The ARIMA analyses are summarized in Table 23.

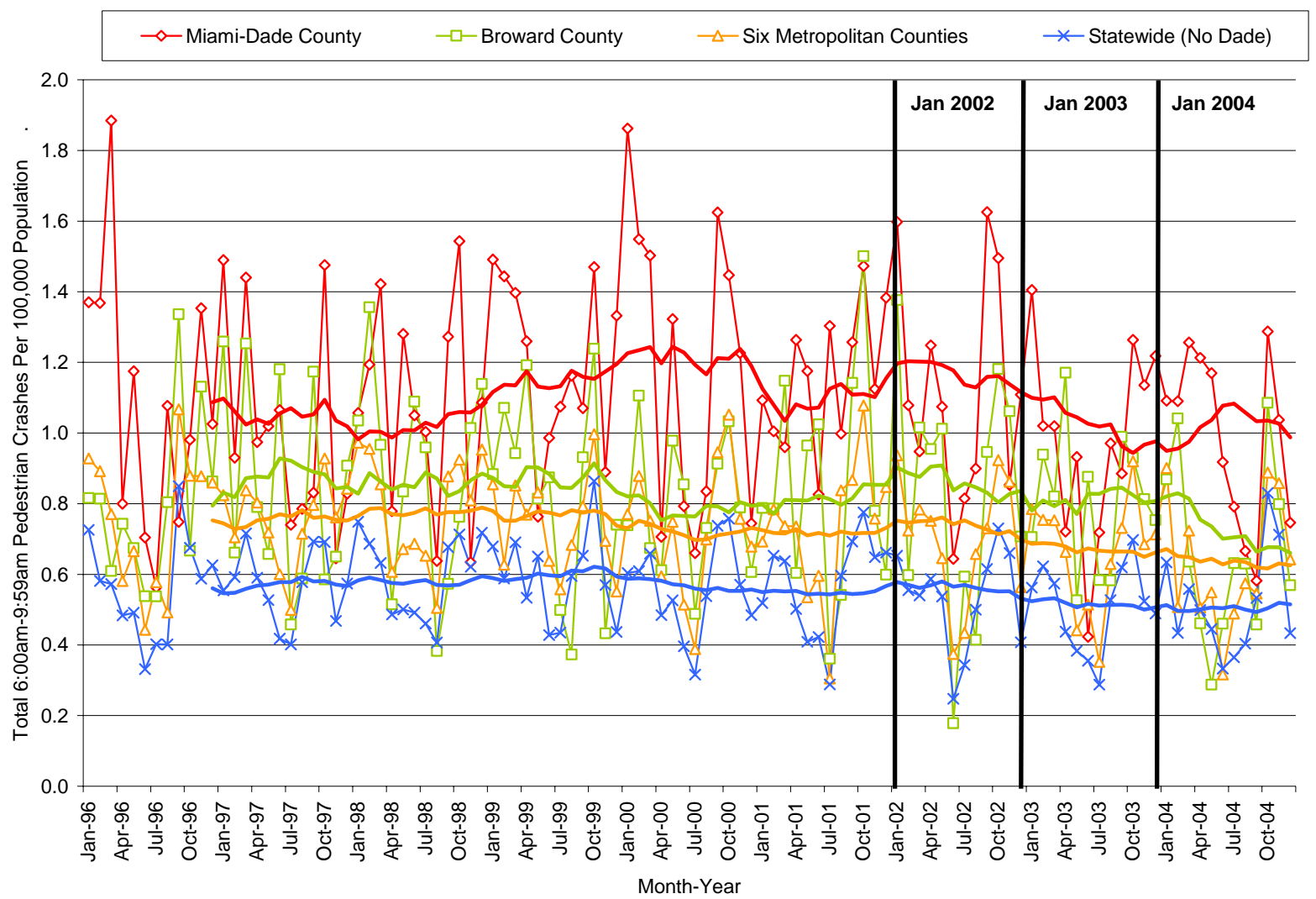

Figure 58. Monthly 6 a.m. to 9:59 a.m. Pedestrian Crashes per 100,000 Population in Miami-Dade County, Broward County, Six Metropolitan Counties (Combined), and Statewide From 1996-2004.

The results of the ARIMA analyses did not indicate a significant change in total pedestrian crashes from 6 a.m. to 9:59 a.m. at any of the intervention points or using any of the control jurisdictions $(p>.05)$. 
Table 22. Unadjusted Post-Intervention Percentage Difference in 6 a.m. to 9:59 a.m. Average Monthly Pedestrian Crash Rates in Each Florida Jurisdiction by Intervention Date, 1996-2004.

\begin{tabular}{|c|c|c|c|}
\hline Jurisdiction & January 2002 & January 2003 & January 2004 \\
\hline Miami-Dade County & -8.4 & -12.4 & -10.4 \\
\hline Broward County & -7.9 & -12.1 & -20.5 \\
\hline Six Metropolitan Counties ${ }^{\mathrm{a}}$ & -11.6 & -13.3 & -14.6 \\
\hline Statewide & -9.7 & -10.0 & -8.0 \\
\hline
\end{tabular}

Table 23. Summary of Sudden-Permanent ARIMA Models of Effect in Miami-Dade County for 6 a.m. to 9:59 a.m. Pedestrian Crash Rates per 100,000 Population Using Three Different Control Series.

\begin{tabular}{|c|c|c|c|c|c|}
\hline Intervention & Model component & Parameter & Lag & Estimate & $t$-value \\
\hline \multicolumn{6}{|c|}{ Broward County Control Series } \\
\hline January 2002 & Intervention & $\omega$ & 0 & -0.0058 & -0.07 \\
\hline January 2003 & Intervention & $\omega$ & 0 & -0.1290 & -1.14 \\
\hline \multirow[t]{3}{*}{ January 2004} & Intervention & $\omega$ & 0 & 0.0625 & 0.55 \\
\hline & Broward Control series & $\beta$ & 0 & 0.3488 & $3.38^{*}$ \\
\hline & Constant & & & 0.8298 & $9.01 *$ \\
\hline \multicolumn{6}{|c|}{ Six Metropolitan Counties Control Series ${ }^{\mathrm{a}}$} \\
\hline January 2002 & Intervention & $\omega$ & 0 & 0.0707 & 0.92 \\
\hline January 2003 & Intervention & $\omega$ & 0 & -0.1059 & -1.06 \\
\hline \multirow[t]{3}{*}{ January 2004} & Intervention & $\omega$ & 0 & 0.0434 & 0.43 \\
\hline & Six Metropolitan Control series & $\beta$ & 0 & 0.9062 & $6.22 *$ \\
\hline & Constant & & & 0.4130 & $3.65^{*}$ \\
\hline \multicolumn{6}{|c|}{ Statewide (Except Miami-Dade) Control Series } \\
\hline January 2002 & Intervention & $\omega$ & 0 & 0.0719 & 0.94 \\
\hline January 2003 & Intervention & $\omega$ & 0 & -0.1099 & -1.10 \\
\hline \multirow[t]{3}{*}{ January 2004} & Intervention & $\omega$ & 0 & 0.0029 & 0.03 \\
\hline & Statewide Control series & $\beta$ & 0 & 1.1700 & $6.28 *$ \\
\hline & Constant & & & 0.4219 & $3.82 *$ \\
\hline
\end{tabular}

Crashes from 10 a.m. to 1:59 p.m. . The pedestrian crash rates during 10 a.m. to 1:59 p.m. are shown in Figure 59, and the raw pre-post percentage differences for each intervention point are shown in Table 24. The ARIMA analyses are summarized in Table 25. 


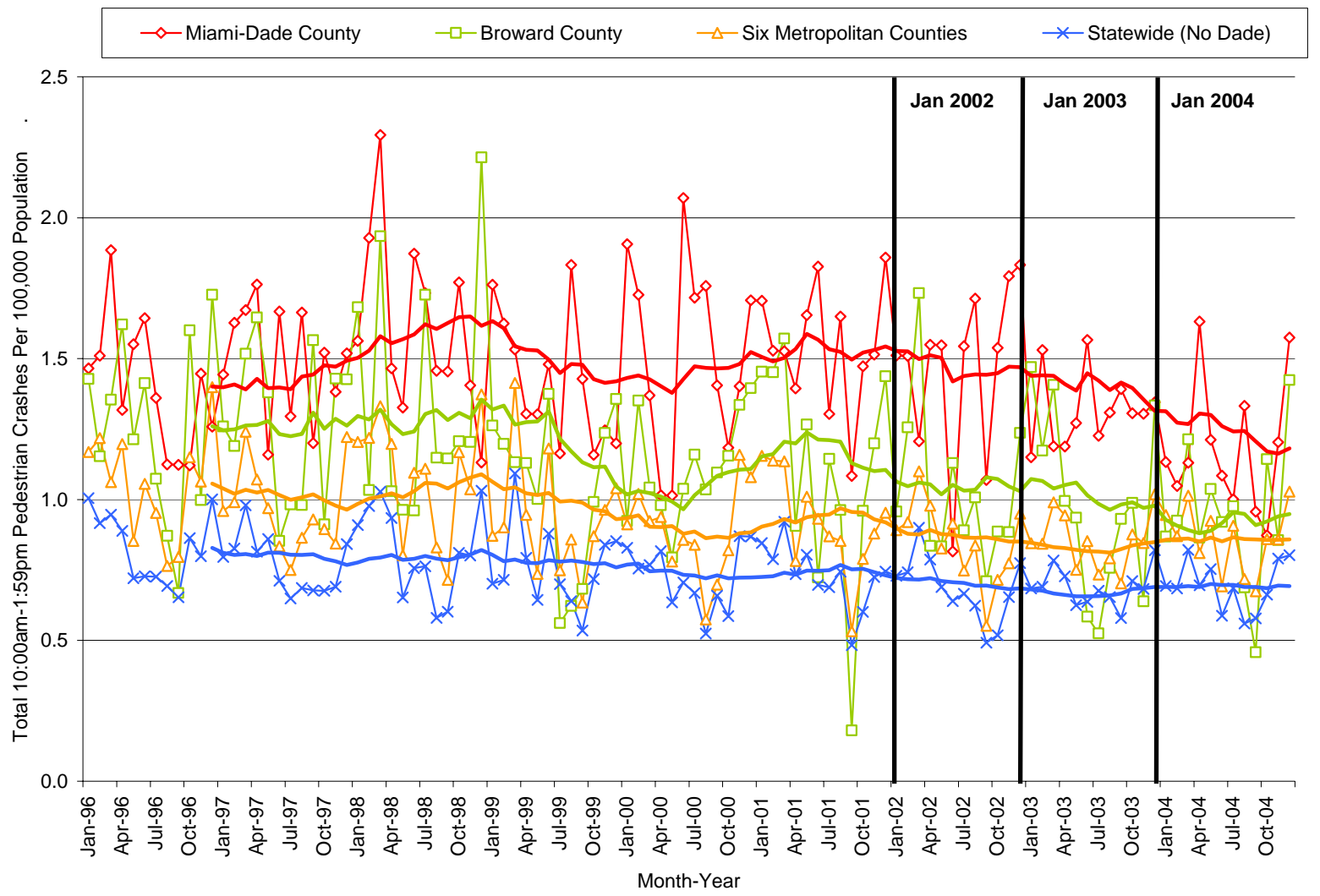

Figure 59. Monthly 10 a.m. to 1:59 p.m. Pedestrian Crashes per 100,000 Population in Miami-Dade County, Broward County, Six Metropolitan Counties (Combined), and Statewide from 1996-2004.

Table 24. Unadjusted Post-Intervention Percentage Difference in 10 a.m. to 1:59 p.m. Average Monthly Pedestrian Crash Rates in Each Florida Jurisdiction by Intervention Date, 1996-2004.

\begin{tabular}{|c|c|c|c|}
\hline Jurisdiction & January 2002 & January 2003 & January 2004 \\
\hline Miami-Dade County & -11.8 & -16.5 & -19.8 \\
\hline Broward County & -17.1 & -17.4 & -17.1 \\
\hline Six Metropolitan Counties ${ }^{\mathrm{a}}$ & -12.5 & -10.7 & -9.0 \\
\hline Statewide & -10.7 & -9.0 & -7.7 \\
\hline
\end{tabular}


Table 25. Summary of Sudden-Permanent ARIMA Models of Effect in Miami-Dade County for 10 a.m. to 1:59 p.m. Pedestrian Crash Rates per 100,000 Population Using Three Different Control Series.

\begin{tabular}{|c|c|c|c|c|c|}
\hline Intervention & Model component & Parameter & Lag & Estimate & t-value \\
\hline \multicolumn{6}{|c|}{ Broward County Control Series } \\
\hline January 2002 & Intervention & $\omega$ & 0 & 0.0972 & 1.83 \\
\hline January 2003 & Intervention & $\omega$ & 0 & -0.2509 & $-3.11^{*}$ \\
\hline \multirow[t]{5}{*}{ January 2004} & Intervention & $\omega$ & 0 & -0.1411 & -1.69 \\
\hline & Noise & MA & 3 & 0.2491 & $2.39^{*}$ \\
\hline & Noise & MA & 9 & 0.3658 & $3.47 *$ \\
\hline & Broward Control series & $\beta$ & 0 & 0.0549 & 0.85 \\
\hline & Constant & & & 1.4202 & $18.07^{*}$ \\
\hline \multicolumn{6}{|c|}{ Six Metropolitan Counties Control Series ${ }^{\mathrm{a}}$} \\
\hline January 2002 & Intervention & $\omega$ & 0 & 0.1073 & 1.93 \\
\hline January 2003 & Intervention & $\omega$ & 0 & -0.2538 & $-3.15^{*}$ \\
\hline \multirow[t]{5}{*}{ January 2004} & Intervention & $\omega$ & 0 & -0.1459 & -1.76 \\
\hline & Noise & MA & 3 & 0.2426 & $2.31^{*}$ \\
\hline & Noise & MA & 9 & 0.3339 & $3.14^{*}$ \\
\hline & Six Metropolitan Control series & $\beta$ & 0 & 0.1826 & 1.58 \\
\hline & Constant & & & 1.3123 & $11.53^{*}$ \\
\hline \multicolumn{6}{|c|}{ Statewide (Except Miami-Dade) Control Series } \\
\hline January 2002 & Intervention & $\omega$ & 0 & 0.0763 & 1.44 \\
\hline January 2003 & Intervention & $\omega$ & 0 & -0.2433 & $-3.14^{*}$ \\
\hline \multirow[t]{5}{*}{ January 2004} & Intervention & $\omega$ & 0 & -0.1466 & -1.83 \\
\hline & Noise & MA & 3 & 0.2395 & $2.25^{*}$ \\
\hline & Noise & MA & 9 & 0.3572 & $3.31 *$ \\
\hline & Statewide Control series & $\beta$ & 0 & 0.2535 & 1.54 \\
\hline & Constant & & & 1.3144 & $10.25^{*}$ \\
\hline
\end{tabular}

The results of the ARIMA analyses for $10 \mathrm{a} . \mathrm{m}$. to 1:59 p.m. indicated a significant reduction in Miami-Dade pedestrian crashes starting January 2003, regardless of the control series used ( $p<$ .05 ). It is interesting to note that none of the control crash series accounted for a significant proportion of the variability in the Miami-Dade crashes for this time period. However, all were kept in the ARIMA models. Using the results with the statewide control series to estimate the effect size, the January 2003 intervention date was associated with a monthly reduction of 0.2 pedestrian crashes per 100,000 population, or about a $16.3 \%$ reduction in the prior pedestrian crash rate during 10 a.m. to 1:59 p.m.

There is no way to pinpoint the reasons why there was a significant reduction in nighttime pedestrian crashes. However, it should be mentioned that there were two specific measures that focused on educating pedestrians on how to walk more safely at night. These included a series of posters to educate pedestrians, developed by Miami-Dade MPO, that were placed on all transit vehicles over several months. The Miami-Dade MPO posters were written in English, Spanish, and Haitian Creole. There were also a separate series of DOT-developed posters that were placed widely in public places and housing developments, etc., throughout the high-crash zones. Both types of posters gave specific details on the nighttime pedestrian crash problem and measures 
that can help pedestrians avoid nighttime crashes (e.g., wear retro-reflective clothing, carry a flashlight, etc.).

Crashes from 2 p.m. to 5:59 p.m.

The pedestrian crash rates during 2 p.m. to 5:59 p.m. are shown in Figure 60, and the raw prepost percentage differences for each intervention point are shown in Table 26. The ARIMA analyses are summarized in Table 27.

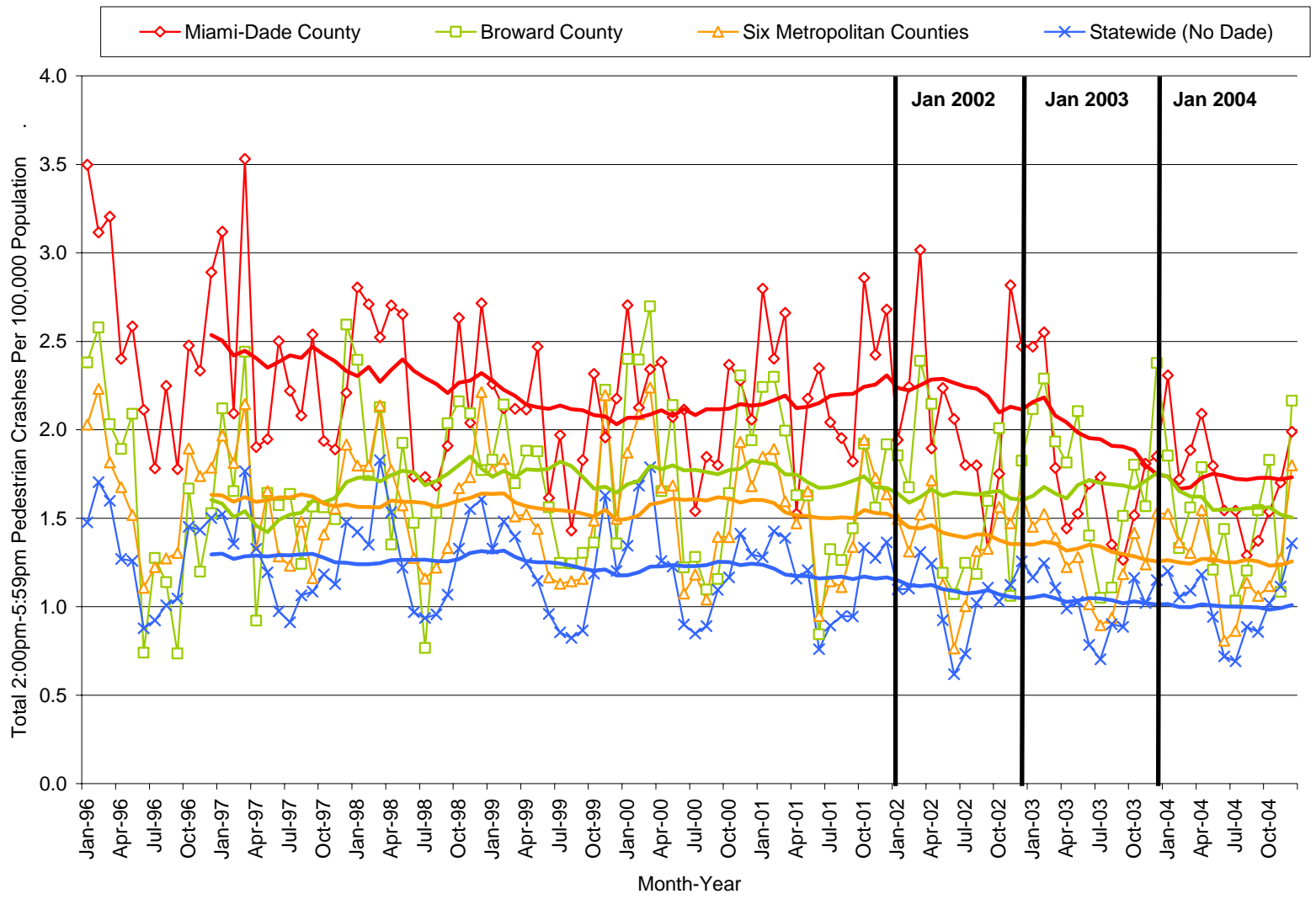

Figure 60. Monthly 2 p.m. to 5:59 p.m. Pedestrian Crashes per 100,000 Population in Miami-Dade County, Broward County, Six Metropolitan Counties (Combined), and Statewide From 1996-2004.

The results of the ARIMA analyses for pedestrian crashes from 2 p.m. to 5:59 p.m. indicated a significant reduction in the pedestrian crash rates at January 2003, regardless of the control series that was used. Using the analysis with the statewide control to characterize the effect, the January 2003 intervention date was associated with a reduction of 0.3 pedestrian crashes per 100,000 population, or about a $13.3 \%$ reduction from the pre-2003 crash series level. 
Table 26. Unadjusted Post-Intervention Percentage Difference in 2 p.m. to 5:59 p.m. Average Monthly Pedestrian Crash Rates in Each Florida Jurisdiction by Intervention Date, 1996-2004.

\begin{tabular}{|c|c|c|c|}
\hline $\begin{array}{rr}\text { Jurisdiction } \\
\end{array}$ & January 2002 & January 2003 & January 2004 \\
\hline Miami-Dade County & -18.1 & -22.8 & -21.0 \\
\hline Broward County & -4.9 & -3.6 & -11.5 \\
\hline Six Metropolitan Counties ${ }^{\mathrm{a}}$ & -18.3 & -18.7 & -16.8 \\
\hline Statewide & -17.5 & -16.7 & -15.0 \\
\hline
\end{tabular}

Table 27. Summary of Sudden-Permanent ARIMA Models of Effect in Miami-Dade County for 2 p.m. to 5:59 p.m. Pedestrian Crash Rates per 100,000 Population Using Three Different Control Series.

\begin{tabular}{|c|c|c|c|c|c|}
\hline Intervention & Model component & Parameter & Lag & Estimate & t-value \\
\hline \multicolumn{6}{|c|}{ Broward County Control Series } \\
\hline January 2002 & Intervention & $\omega$ & 0 & -0.1481 & -1.31 \\
\hline January 2003 & Intervention & $\omega$ & 0 & -0.4165 & $-3.11 *$ \\
\hline \multirow[t]{4}{*}{ January 2004} & Intervention & $\omega$ & 0 & 0.0940 & 0.69 \\
\hline & Noise & MA & 12 & -0.2150 & $-2.20 *$ \\
\hline & Broward Control series & $\beta$ & 0 & 0.4433 & $5.34 *$ \\
\hline & Constant & & & 1.5354 & $10.25^{*}$ \\
\hline \multicolumn{6}{|c|}{ Six Metropolitan Counties Control Series ${ }^{\mathrm{a}}$} \\
\hline January 2002 & Intervention & $\omega$ & 0 & -0.0164 & -0.15 \\
\hline January 2003 & Intervention & $\omega$ & 0 & -0.2690 & $-2.13 *$ \\
\hline \multirow[t]{4}{*}{ January 2004} & Intervention & $\omega$ & 0 & -0.0190 & -0.15 \\
\hline & Noise & MA & 12 & -0.2121 & $-2.20 *$ \\
\hline & Six Metropolitan Control series & $\beta$ & 0 & 0.7884 & $6.74 *$ \\
\hline & Constant & & & 1.0445 & $5.48^{*}$ \\
\hline \multicolumn{6}{|c|}{ Statewide (Except Miami-Dade) Control Series } \\
\hline January 2002 & Intervention & $\omega$ & 0 & 0.0087 & 0.08 \\
\hline January 2003 & Intervention & $\omega$ & 0 & -0.2994 & $-2.41 *$ \\
\hline \multirow[t]{4}{*}{ January 2004} & Intervention & $\omega$ & 0 & -0.0156 & -0.13 \\
\hline & Noise & MA & 12 & -0.2119 & $-2.19 *$ \\
\hline & Statewide Control series & $\beta$ & 0 & 1.0851 & $7.16^{*}$ \\
\hline & Constant & & & 0.9421 & $4.87^{*}$ \\
\hline
\end{tabular}

Crashes from 6 p.m. to 9:59 p.m.

The pedestrian crash rates during 6 p.m. to 9:59 p.m. are shown in Figure 61, and the raw prepost percentage differences for each intervention point are shown in Table 28. The ARIMA analyses are summarized in Table 29. 


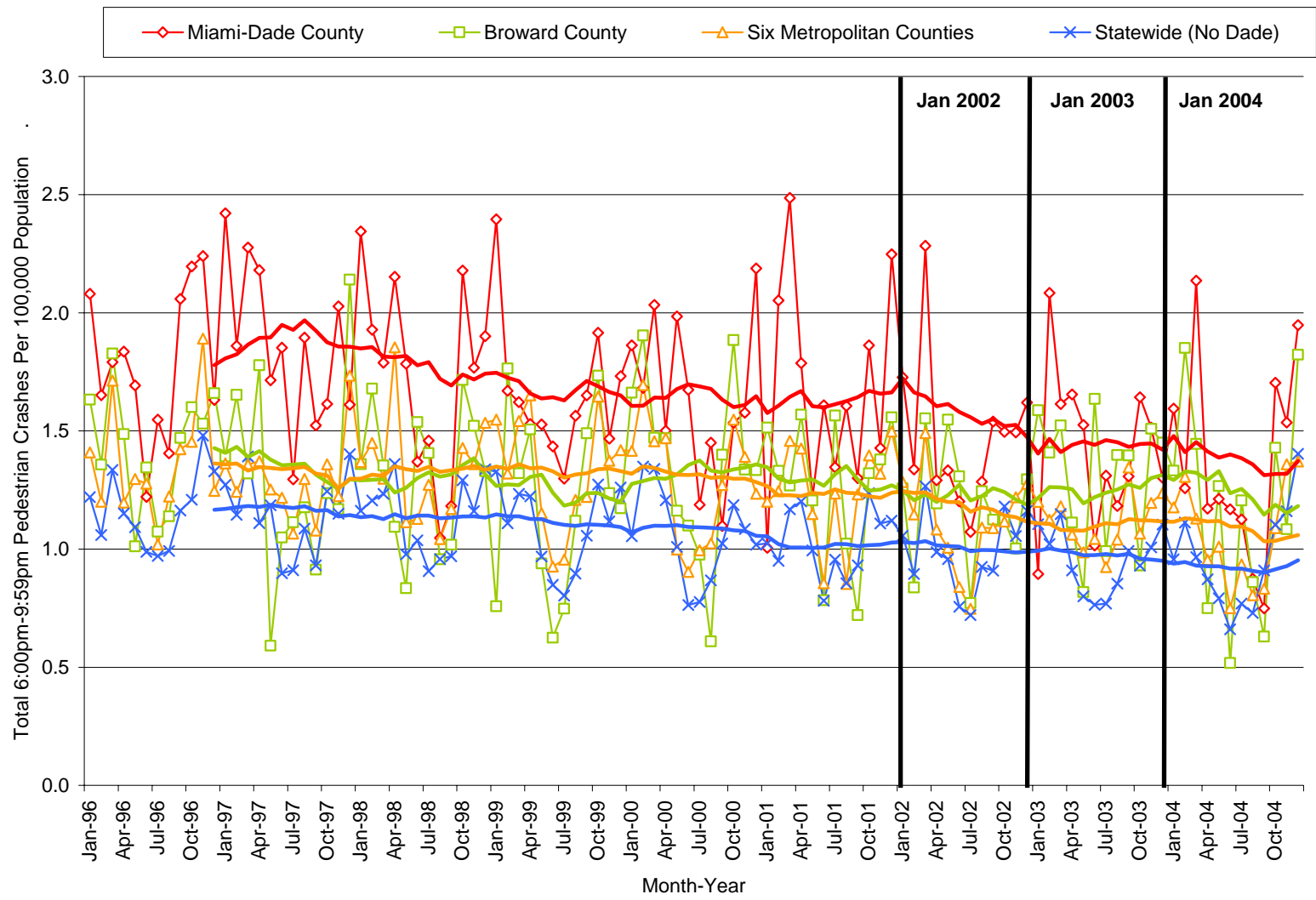

Figure 61. Monthly 6 p.m. to 9:59 p.m. Pedestrian Crashes per 100,000 Population in Miami-Dade County, Broward County, Six Metropolitan Counties (Combined), and Statewide from 1996-2004.

Table 28. Unadjusted Post-Intervention Percentage Difference in 6 p.m. to 9:59 p.m. Average Monthly Pedestrian Crash Rates in Each Florida Jurisdiction by Intervention Date, 1996-2004.

\begin{tabular}{|l|c|c|c|}
\hline \multicolumn{1}{|c|}{ Jurisdiction } & January 2002 & January 2003 & January 2004 \\
\hline Miami-Dade County & -17.5 & -17.2 & -17.0 \\
\hline Broward County & -6.4 & -3.5 & -8.8 \\
\hline Six Metropolitan Counties ${ }^{\text {a }}$ & -16.1 & -15.0 & -15.9 \\
\hline Statewide & -12.6 & -12.5 & -10.9 \\
\hline $\begin{array}{l}\text { Note. Each percentage difference represents the difference in the average monthly pedestrian crash rate per } \\
\text { 100,000 population in the post-intervention period relative to the pre-intervention average monthly rate. } \\
\text { a Duval, Hillsborough, Pinellas, Palm Beach, Orange, and Broward counties. }\end{array}$ \\
\hline
\end{tabular}


Table 29. Summary of Sudden-Permanent ARIMA Models of Effect in Miami-Dade County for 6 p.m. to 9:59 p.m. Pedestrian Crash Rates per 100,000 Population Using Three Different Control Series.

\begin{tabular}{|c|c|c|c|c|c|}
\hline Intervention & Model component & Parameter & Lag & Estimate & t-value \\
\hline \multicolumn{6}{|c|}{ Broward County Control Series } \\
\hline January 2002 & Intervention & $\omega$ & 0 & -0.2335 & $-3.62 *$ \\
\hline January 2003 & Intervention & $\omega$ & 0 & -0.0529 & -0.64 \\
\hline \multirow[t]{5}{*}{ January 2004} & Intervention & $\omega$ & 0 & -0.0404 & -0.48 \\
\hline & Noise & MA & 4 & 0.4838 & $4.91^{*}$ \\
\hline & Noise & MA & 12 & -0.2944 & $-2.49^{*}$ \\
\hline & Broward Control series & $\beta$ & 0 & 0.2378 & $2.73 *$ \\
\hline & Constant & & & 1.4021 & $12.03 *$ \\
\hline \multicolumn{6}{|c|}{ Six Metropolitan Counties Control Series ${ }^{\mathrm{a}}$} \\
\hline January 2002 & Intervention & $\omega$ & 0 & -0.0917 & -0.94 \\
\hline January 2003 & Intervention & $\omega$ & 0 & -0.0549 & -0.45 \\
\hline \multirow[t]{3}{*}{ January 2004} & Intervention & $\omega$ & 0 & 0.0017 & 0.01 \\
\hline & Six Metropolitan Control series & $\beta$ & 0 & 0.8272 & $5.88^{*}$ \\
\hline & Constant & & & 0.6413 & $3.42 *$ \\
\hline \multicolumn{6}{|c|}{ Statewide (Except Miami-Dade) Control Series } \\
\hline January 2002 & Intervention & $\omega$ & 0 & -0.1133 & -1.59 \\
\hline January 2003 & Intervention & $\omega$ & 0 & 0.0145 & 0.16 \\
\hline \multirow[t]{4}{*}{ January 2004} & Intervention & $\omega$ & 0 & -0.0723 & -0.79 \\
\hline & Noise & MA & 1 & 0.2262 & $2.40^{*}$ \\
\hline & Statewide Control series & $\beta$ & 0 & 1.2287 & $8.72 *$ \\
\hline & Constant & & & 0.3661 & $2.32 *$ \\
\hline
\end{tabular}

The ARIMA models using the six metropolitan counties and the statewide control did not indicate a significant change in the Miami-Dade County crash rate from 6 p.m. to 9:59 p.m. at any of the intervention points $(p>.05)$. Broward County experienced an apparent increase in pedestrian crashes from 6 p.m. to $9: 59$ p.m. in the post period, which may have occurred in Miami-Dade County if the pedestrian safety program had not been implemented. If one believes this is true, the significant effect in the model with the Broward County control series indicates a reduction of 0.20 crashes per 100,000 population starting in January 2002. This is approximately a $13.6 \%$ reduction from the pre-2002 average series level.

Crashes from 10 p.m. to 5:59 a.m.

The pedestrian crash rates during 10 p.m. to 5:59 a.m. are shown in Figure 62, and the raw prepost percentage differences for each intervention point are shown in Table 30. The ARIMA analyses are summarized in Table 31. 


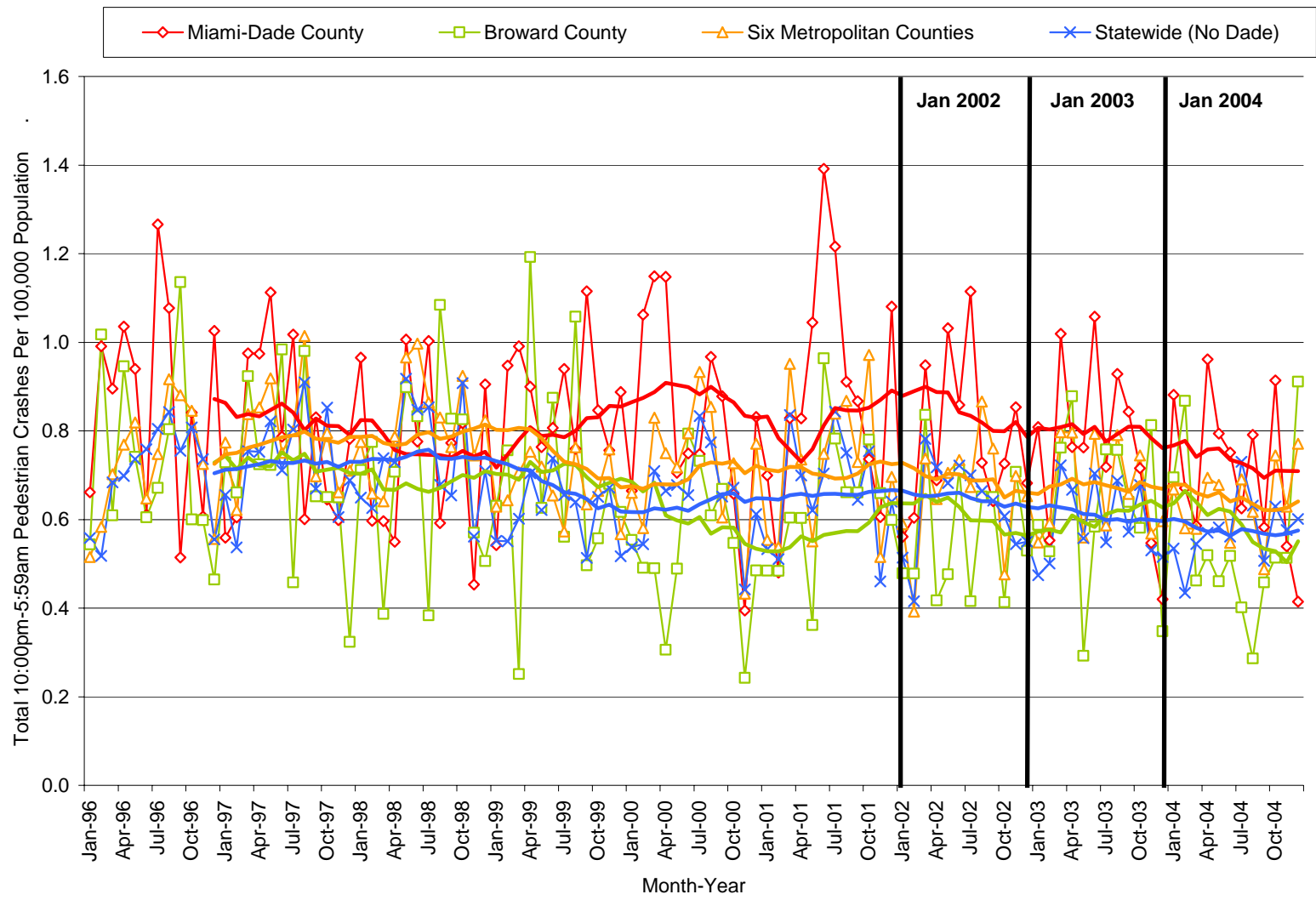

Figure 62. Monthly 10 p.m. to 5:59 a.m. Pedestrian Crashes per 100,000 Population in Miami-Dade County, Broward County, Six Metropolitan Counties (Combined), and Statewide from 1996-2004.

Table 30. Unadjusted Post-Intervention Percentage Difference in 10 p.m. to 5:59 a.m. Average Monthly Pedestrian Crash Rates in Each Florida Jurisdiction by Intervention Date, 1996-2004.

\begin{tabular}{|c|c|c|c|}
\hline Jurisdiction & January 2002 & January 2003 & January 2004 \\
\hline Miami-Dade County & -9.5 & -10.9 & -13.2 \\
\hline Broward County & -13.0 & -9.8 & -15.2 \\
\hline Six Metropolitan Counties ${ }^{\mathrm{a}}$ & -11.3 & -10.2 & -11.2 \\
\hline Statewide & -12.3 & -13.4 & -13.7 \\
\hline
\end{tabular}


Table 31. Summary of Sudden-Permanent ARIMA Models of Effect in Miami-Dade County for 10 p.m. to 5:59 a.m. Pedestrian Crash Rates per 100,000 Population Using Three Different Control Series.

\begin{tabular}{|c|c|c|c|c|c|}
\hline Intervention & Model component & Parameter & Lag & Estimate & t-value \\
\hline \multicolumn{6}{|c|}{ Broward County Control Series } \\
\hline January 2002 & Intervention & $\omega$ & 0 & -0.0266 & -0.44 \\
\hline January 2003 & Intervention & $\omega$ & 0 & -0.0271 & -0.40 \\
\hline \multirow[t]{4}{*}{ January 2004} & Intervention & $\omega$ & 0 & -0.0535 & -0.76 \\
\hline & Noise & MA & 12 & -0.3042 & $-2.94^{*}$ \\
\hline & Broward Control series & $\beta$ & 0 & -0.0041 & -0.04 \\
\hline & Constant & & & 0.8177 & $11.33 *$ \\
\hline \multicolumn{6}{|c|}{ Six Metropolitan Counties Control Series ${ }^{\mathrm{a}}$} \\
\hline January 2002 & Intervention & $\omega$ & 0 & -0.0093 & -0.15 \\
\hline January 2003 & Intervention & $\omega$ & 0 & -0.0258 & -0.37 \\
\hline \multirow[t]{4}{*}{ January 2004} & Intervention & $\omega$ & 0 & -0.0454 & -0.65 \\
\hline & Noise & MA & 12 & -0.2445 & $-2.37^{*}$ \\
\hline & Six Metropolitan Control series & $\beta$ & 0 & 0.2682 & 1.71 \\
\hline & Constant & & & 0.6169 & $5.17 *$ \\
\hline \multicolumn{6}{|c|}{ Statewide (Except Miami-Dade) Control Series } \\
\hline January 2002 & Intervention & $\omega$ & 0 & -0.0074 & -0.12 \\
\hline January 2003 & Intervention & $\omega$ & 0 & -0.0079 & -0.11 \\
\hline \multirow[t]{4}{*}{ January 2004} & Intervention & $\omega$ & 0 & -0.0437 & -0.62 \\
\hline & Noise & MA & 12 & -0.2076 & $-1.97^{*}$ \\
\hline & Statewide Control series & $\beta$ & 0 & 0.4406 & $2.28^{*}$ \\
\hline & Constant & & & 0.5139 & $3.82 *$ \\
\hline
\end{tabular}

None of the ARIMA models indicated a significant change in Miami-Dade County pedestrian crashes from 10 p.m. to 5:59 a.m. at any of the intervention points $(p>.05)$. It does not appear that the implementation of the pedestrian safety project was associated with reductions in pedestrian crashes during this time of night.

\section{Zone-by-Zone Crash Analysis}

As described in Chapter 3, four high-pedestrian-crash zones within Miami-Dade County were identified and targeted for specialized interventions based on the particular characteristics of their pedestrian crash problem. This section reports on an analysis of the effectiveness of the intervention program implemented in each of the four zones (see Figure 63). 

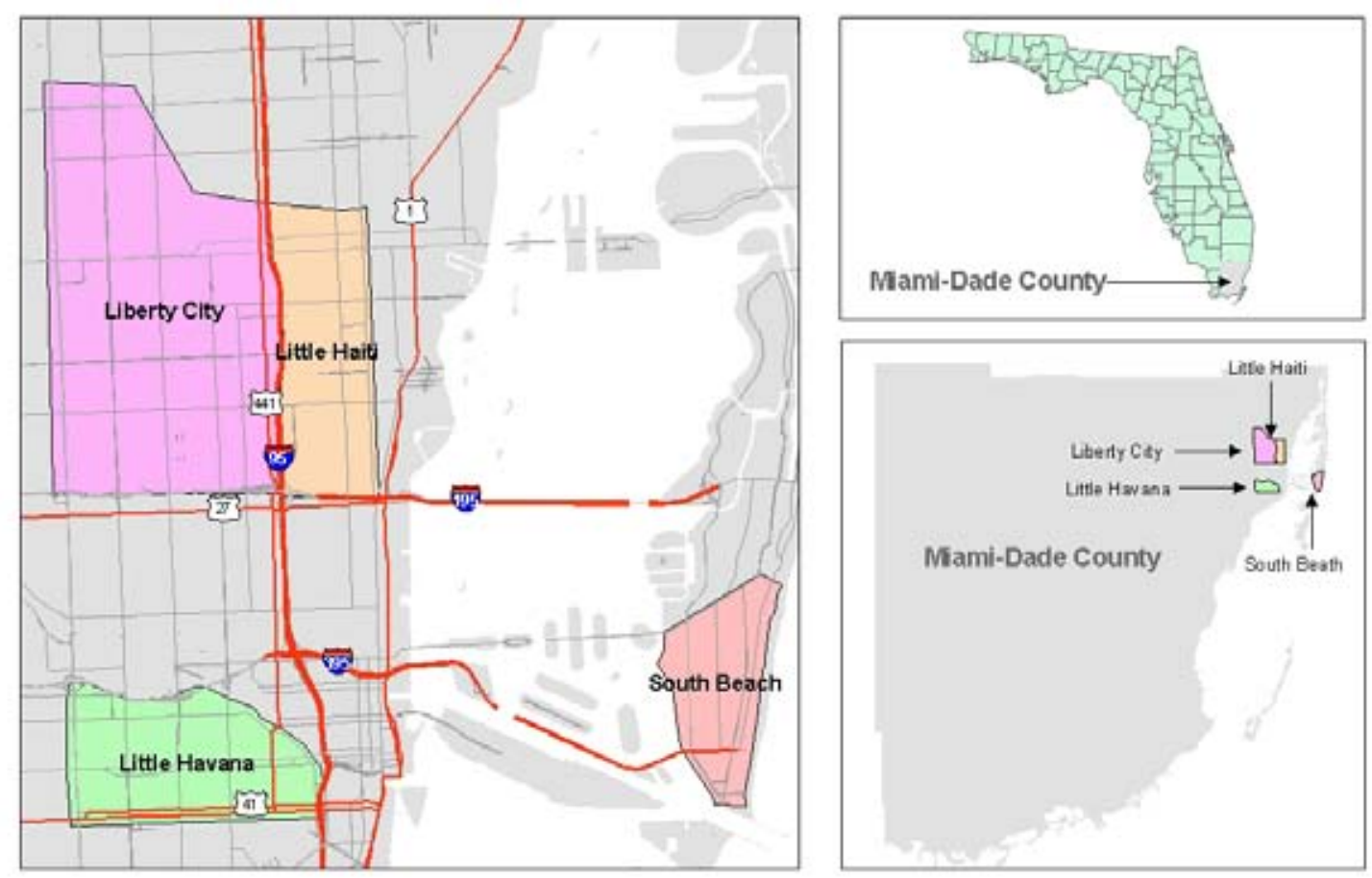

Figure 63. Crash Zone Locations in Miami-Dade County, Florida.

Table 32 charts the number of reported pedestrian crashes in each of the four target zones and countywide over the duration of the project. To assess the safety effects of the countermeasure programs implemented, the project team relied on 1996 to 2004 pedestrian crash data provided by the Miami-Dade County MPO that was subsequently geocoded and crash typed. The period from 1996 to 2001 was identified as the pre-program period, and 2002 to 2004 as the postprogram period, since the countermeasure program became fully active in 2002 . Two analytical methods were employed. The first analysis was a spatial analysis of crash density, which mapped out the spatial distribution of pedestrian crash locations and demonstrated concentrations of pedestrian crashes based on estimated density values. The second analysis was a descriptive analysis of crash frequency, which compared the total numbers of pedestrian crashes and the monthly number of pedestrian crashes across zones. These two types of analyses are described in the following pages. It should be noted that the pedestrian crash database used in the previous analysis included Miami-Dade pedestrian crashes taken from the official FDOT motor vehicle computerized database, containing crashes from 1996 to 2004. The database used in the zone analysis was determined by geocoding all pedestrian crashes from police reports of pedestrian crashes. Not all of the pedestrian crashes could be located precisely for geocoding purposes. 
Table 32. Pedestrian Crash Frequency by Crash Zones (1996-2004).

\begin{tabular}{|l|c|c|c|c|c|}
\hline Year & Countywide & Liberty City & Little Haiti & Little Havana & South Beach \\
\hline 1996 & 1,789 & 134 & 63 & 91 & 111 \\
\hline 1997 & 1,753 & 137 & 53 & 65 & 106 \\
\hline 1998 & 1,604 & 121 & 53 & 84 & 86 \\
\hline 1999 & 1,636 & 107 & 48 & 57 & 101 \\
\hline 2000 & 1,784 & 112 & 62 & 83 & 103 \\
\hline 2001 & 1,921 & 122 & 62 & 98 & 89 \\
\hline 2002 & 1,783 & 111 & 70 & 75 & 87 \\
\hline 2003 & 1,604 & 74 & 49 & 88 & 74 \\
\hline 2004 & 1,598 & 93 & 53 & 82 & 74 \\
\hline Total & $\mathbf{1 5 , 4 7 2}$ & $\mathbf{1 , 0 1 1}$ & $\mathbf{5 1 3}$ & $\mathbf{7 2 3}$ & $\mathbf{8 3 1}$ \\
\hline
\end{tabular}

\section{Spatial Analysis of Crash Density}

The spatial analysis was based on the existing geocoded crash files provided by Miami-Dade County MPO described earlier in this report. In addition to detailed description about each pedestrian crash, those files plot the specific locations of all pedestrian crashes in Miami-Dade County during the years 1996-2004.

The presence of the exact crash locations on a GIS map shows spatial clustering and dispersion across the study area. However, a standard GIS map cannot reflect the crash concentration of locations having multiple crashes, since several crashes may have occurred the same location. In this research, high crash concentrations within each of the four zones were identified by developing crash density estimates.

Two software tools were used for the spatial analyses-CrimeStat 3.0 and ArcGIS 9.1. CrimeStat is a spatial statistics program for the analysis of crime incident locations, developed by Ned Levine \& Associates (Levine, 2004). In this analysis, pedestrian crashes were defined as the incidents, instead of crimes. ArcGIS is an integrated collection of GIS software products for compiling, authoring, analyzing, mapping and publishing geographic information, developed by the Environmental Systems Research Institute, Inc. The general steps in the spatial analysis included generating crash density estimates using CrimeStat 3.0 and displaying crash density estimates using ArcGIS 9.1. A table of the pedestrian crash counts by age group, zone, and month used for this analysis is provided in Appendix C.

\section{$\underline{\text { Generate Crash Density Estimates Using CrimeStat 3.0 }}$}

CrimeStat provides several tools for identifying clusters and concentrations of crash incidents, including four hot spot techniques (the mode, fuzzy mode, nearest neighbor hierarchical clustering, and risk adjusted nearest neighbor hierarchical clustering) and several kernel density interpolation techniques (adaptive/fixed bandwidth; normal/uniform/quadratic/triangular/peaked distribution). While hot spot analysis identifies cluster areas of incidents, kernel density interpolation generates incident density estimates for all parts of a region (at any location). In other words, kernel density interpolation is able to quantify the extent of crash concentration at 
any location and the resulting density map is similar to a contour map. Hot spot analysis can only identify the locations of 'hot spot' areas (crash clustering areas). This research used kernel density interpolation to identify high-crash zones, since it provides more comprehensive information about the spatial crash patterns. Detailed descriptions about kernel density estimation (data setups, method selection criteria, output units, etc.) can be found in CrimeStat III: A Spatial Statistics Program for the Analysis of Crime Incident Locations (Levine, 2004).

The bandwidth of the applied kernel density function will affect the results of density estimation. The size of bandwidth will determine the smoothness of the estimated density surface. A narrow bandwidth would be good for identifying local clusters, while a larger bandwidth would be for capturing major trends in the data. Since this research aimed at examining the general spatial patterns of pedestrian crashes in four crash zones and countywide area in Miami-Dade County, relatively large bandwidths corresponding to the size of the zones were used. Kernel functions with bandwidths of 0.5 mile and 1.0 mile were used to estimate the crash density surface for the countywide area, while the kernel function with a bandwidth of 0.2 mile was used for each of the four identified crash zones.

\section{Display Crash Density Estimates Using ArcGIS 9.1}

CrimeStat is able to output the estimated crash density values into ArcGIS files, which provides an opportunity to visually present the density estimates using ArcGIS. The density estimates are scaled by color. Darker tones represent higher densities while lighter tones represent lower densities. Scales are also provided on each map to indicate crash density for each color code.

Figure 64 and Figure 65 present the countywide spatial distribution of pedestrian crashes. The crash density surface in Figure 64 was estimated using kernel function with a bandwidth of 0.5 mile. The density estimate illustrated in Figure 65 used the kernel function with a 1.0-mile bandwidth. Figure 65 illustrates the general countywide pattern better, while Figure 64 identifies the local hot spots better.

Figure 64 indicates that, in Liberty City and South Beach, locations with higher crash concentrations declined from 1996 to 2004. However, the changes in spatial distribution of pedestrian crashes are not notable in Little Haiti and Little Havana.

Figure 65 presents the geographic patterns of pedestrian crashes in Miami-Dade County over time. The overall crash concentrations in Miami-Dade County decreased from 1996 to 2004, as noted from the lesser amount of darker (orange and red) colors in 2003 and 2004, compared to most of the earlier years.

Figure 66 displays the spatial concentrations of pedestrian crashes in the four crash zones. The next Figures present the spatial patterns in Liberty City (Figure 67), Little Haiti (Figure 68), Little Havana (Figure 69), and South Beach (Figure 70). Those figures indicate the general declining trends of crash concentrations in all four zones. The amount of decrease is greater in South Beach and Liberty City than in Little Havana and Little Haiti. 


\section{Miami-Dade County Pedestrian Crash Density Map (1996-2004)}

Kernel density estimation; bandwidth $=0.5$ mile

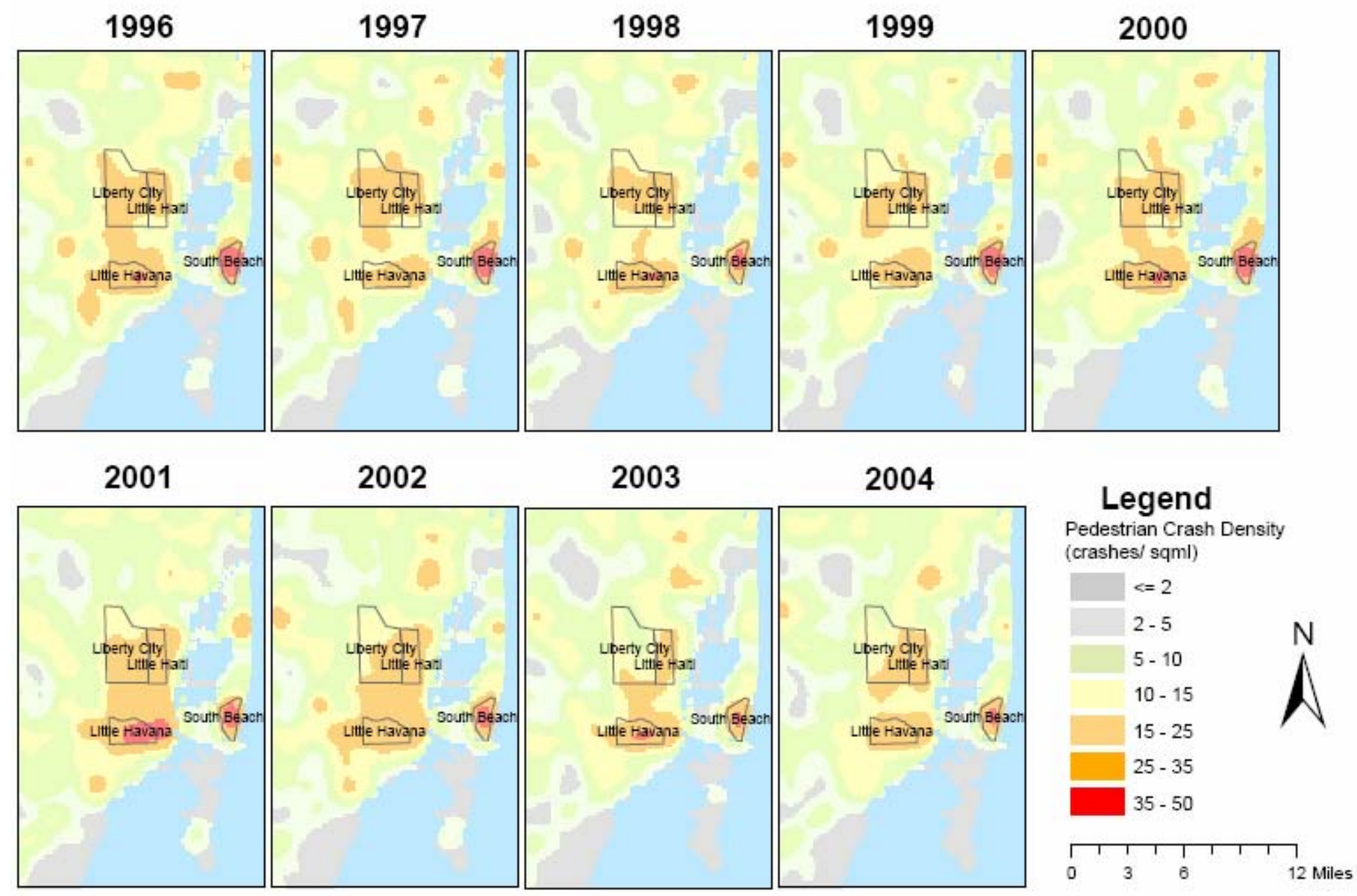

Figure 64. Miami-Dade County Pedestrian Crash Density Map (Kernel density estimation; bandwidth = 0.5 mile) 
Miami-Dade County Pedestrian Crash Density Map (1996-2004)

Kernel density estimation; bandwidth $=1.0$ mile

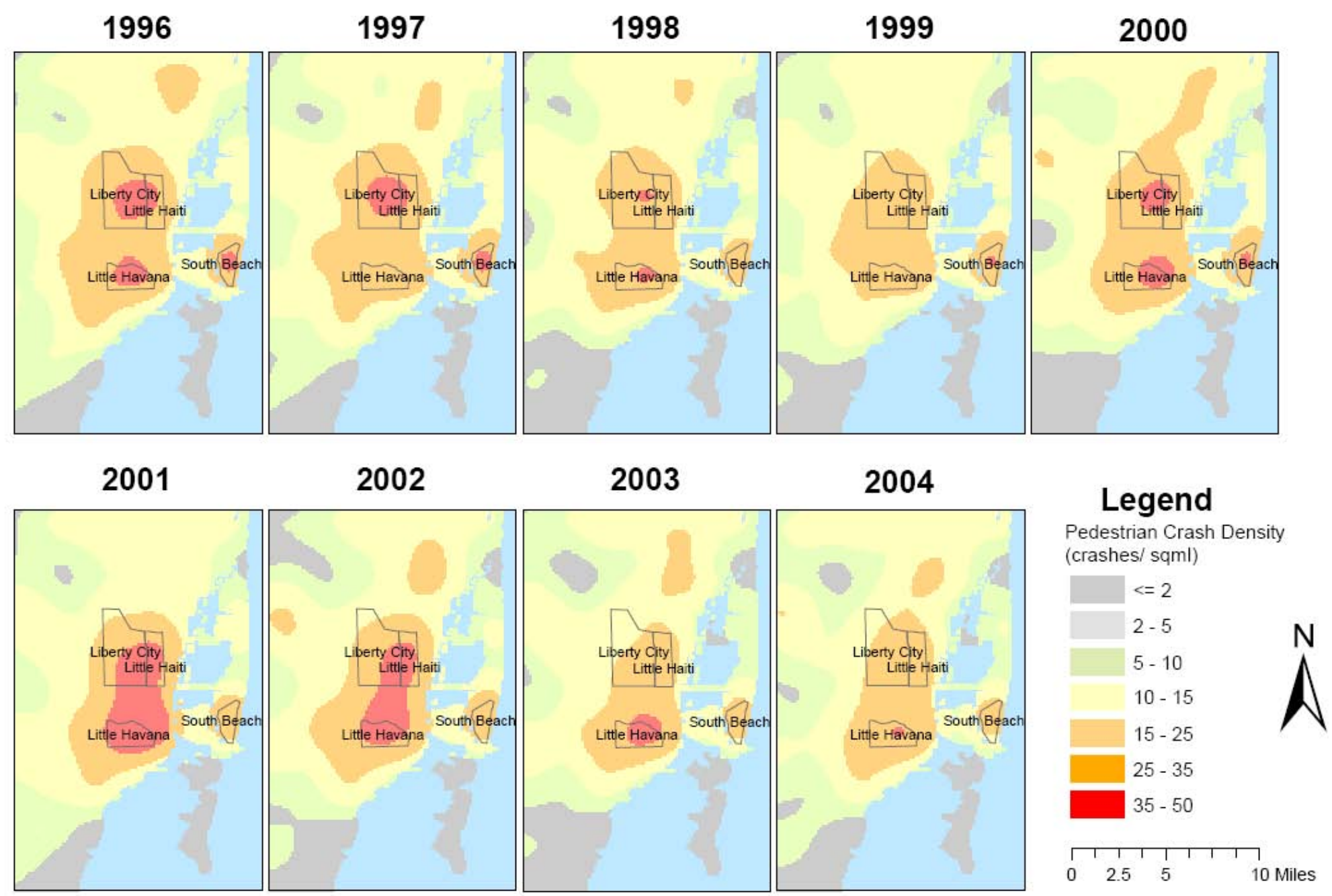

Figure 65. Miami-Dade County Pedestrian Crash Density Map (Kernel density estimation; bandwidth = 1.0 mile). 


\section{Miami-Dade County Pedestrian Crash Zone Density Map (1996-2004)}

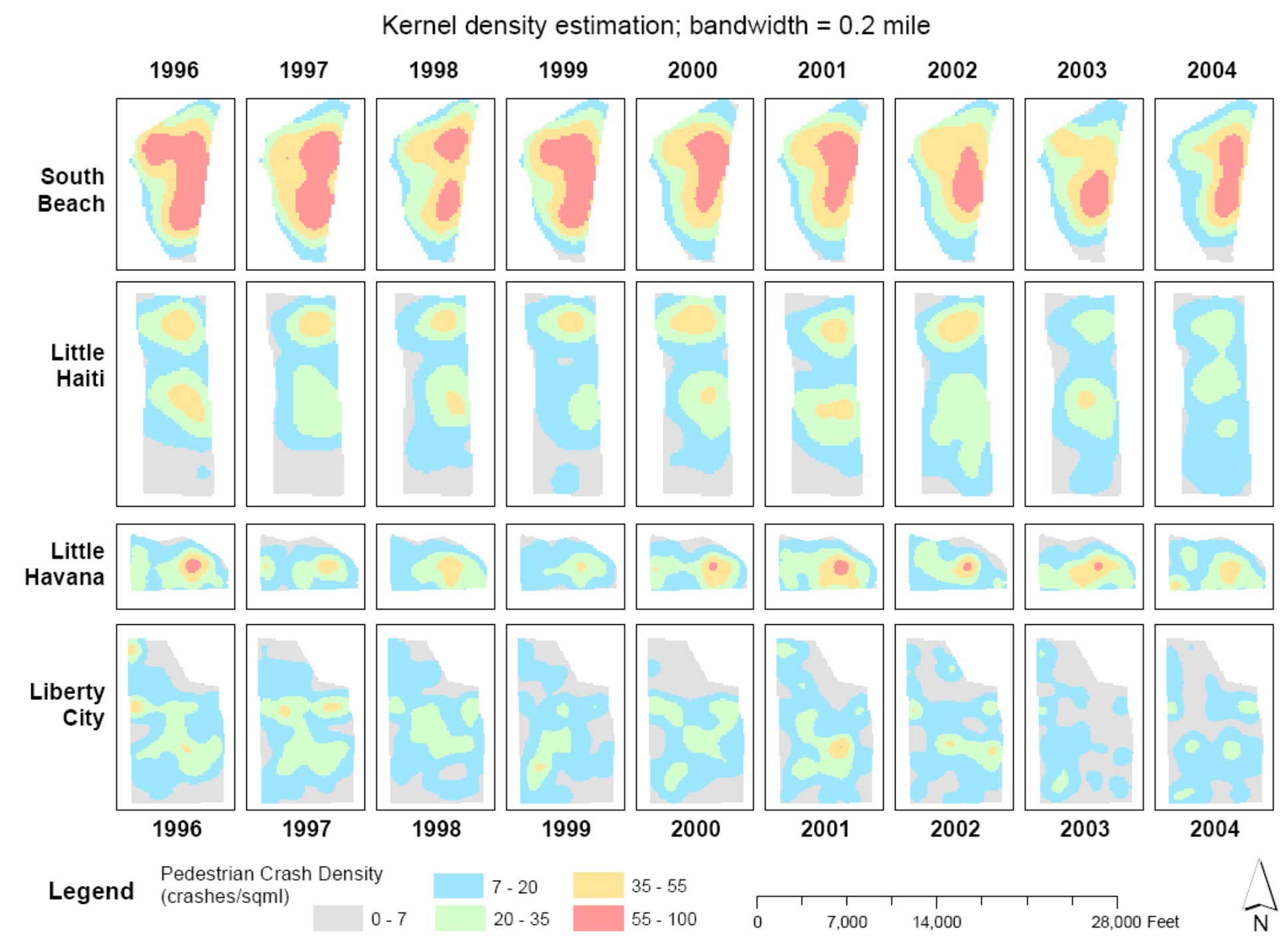

Figure 66. Crash Density Map of Four Crash Zones (Kernel density estimation; bandwidth $=0.2$ mile). 


\section{Liberty City Pedestrian Crash Zone Density Map (1996-2004)}

Kernel density estimation; bandwidth $=0.2$ mile
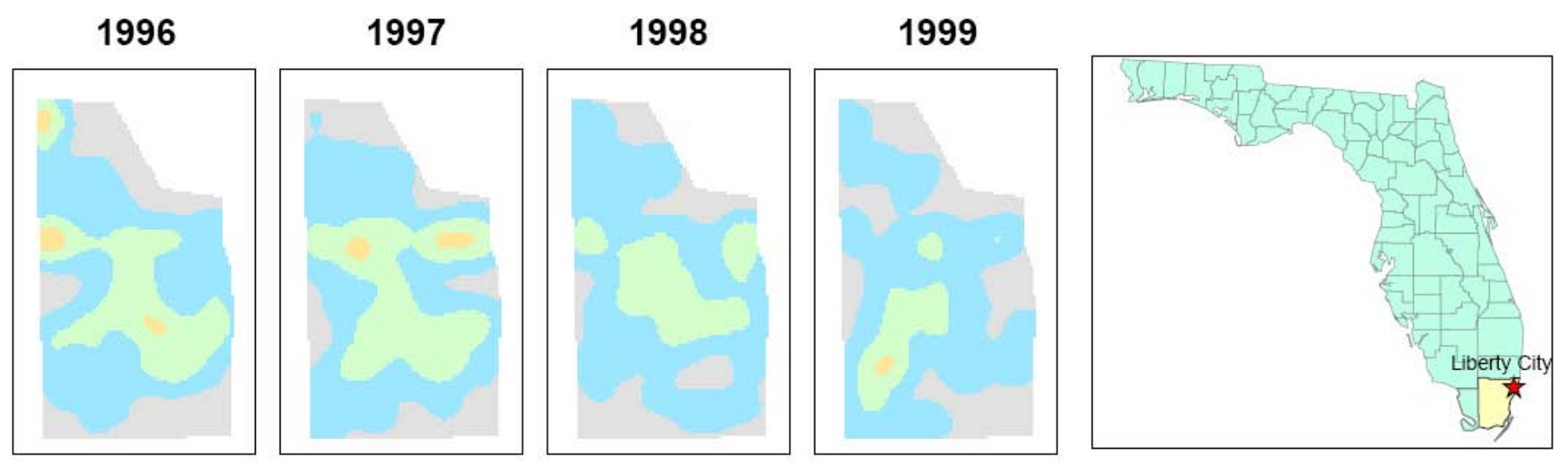

2000
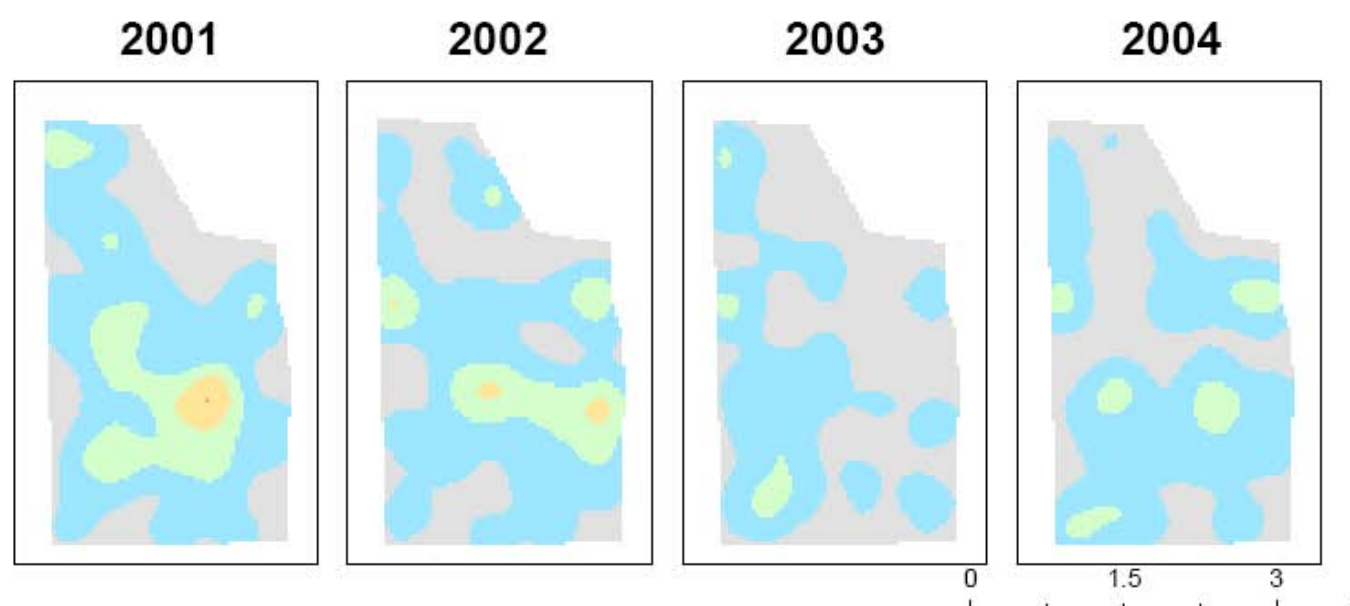

\section{Legend}

\section{Crash Density} (crashes/sqml)
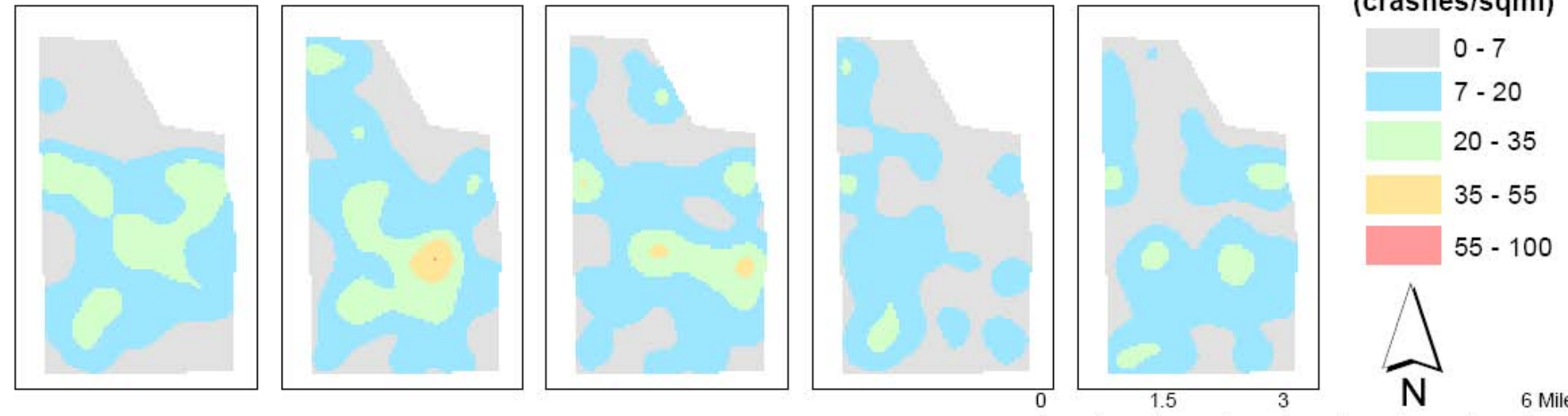

Figure 67. Liberty City Crash Density Map (Kernel density estimation; bandwidth $=0.2$ mile). 
Little Haiti Pedestrian Crash Zone Density Map (1996-2004)

Kernel density estimation; bandwidth $=0.2$ mile
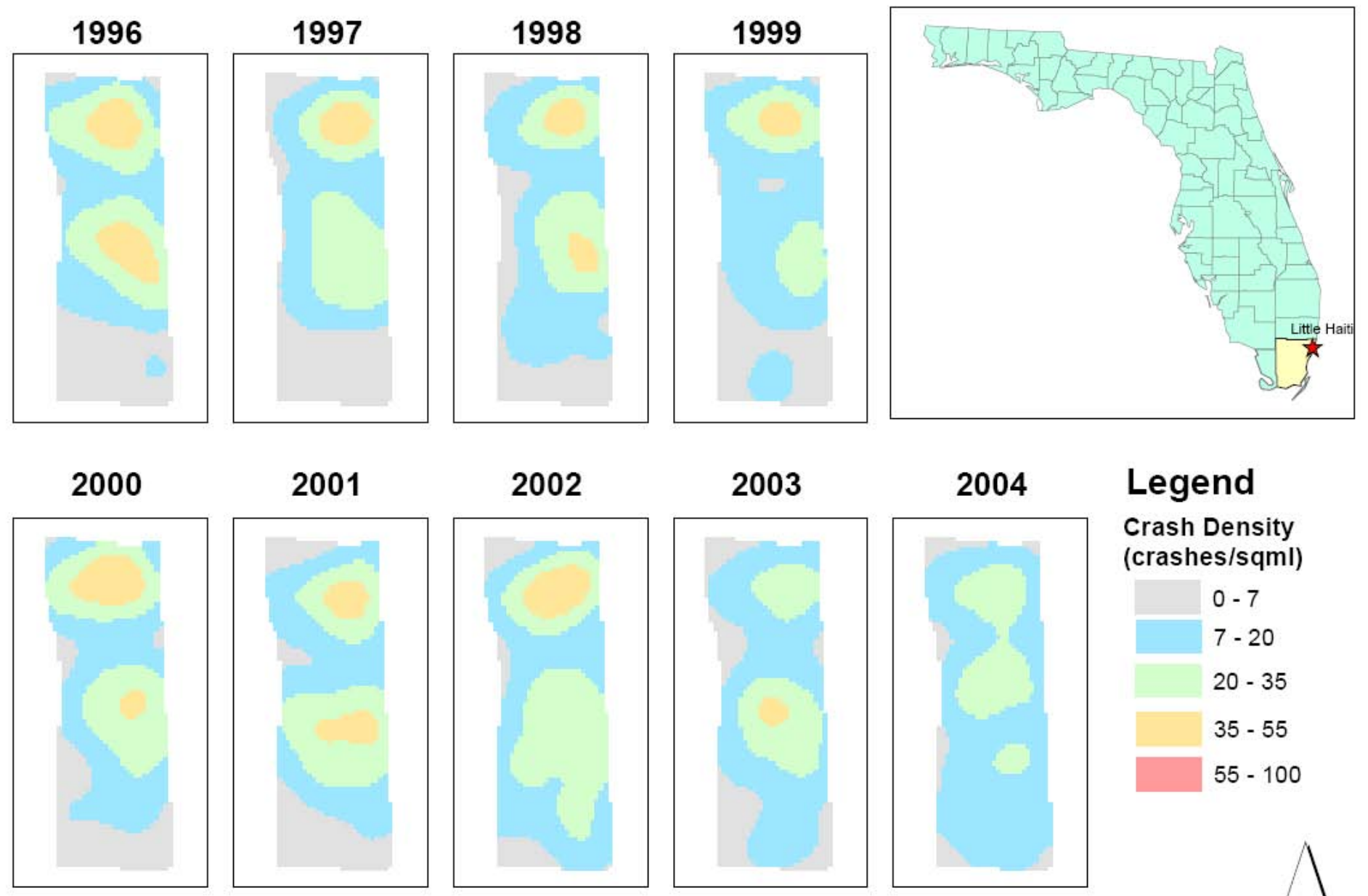

Crash Density (crashes/sqml)

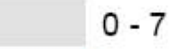

$7-20$

$20-35$

$35-55$

$55-100$

0 2 4 Miles

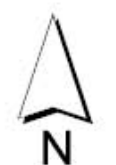

Figure 68. Little Haiti Crash Density Map (Kernel density estimation; bandwidth = 0.2 mile). 


\section{Little Havana Pedestrian Crash Zone Density Map (1996-2004)}

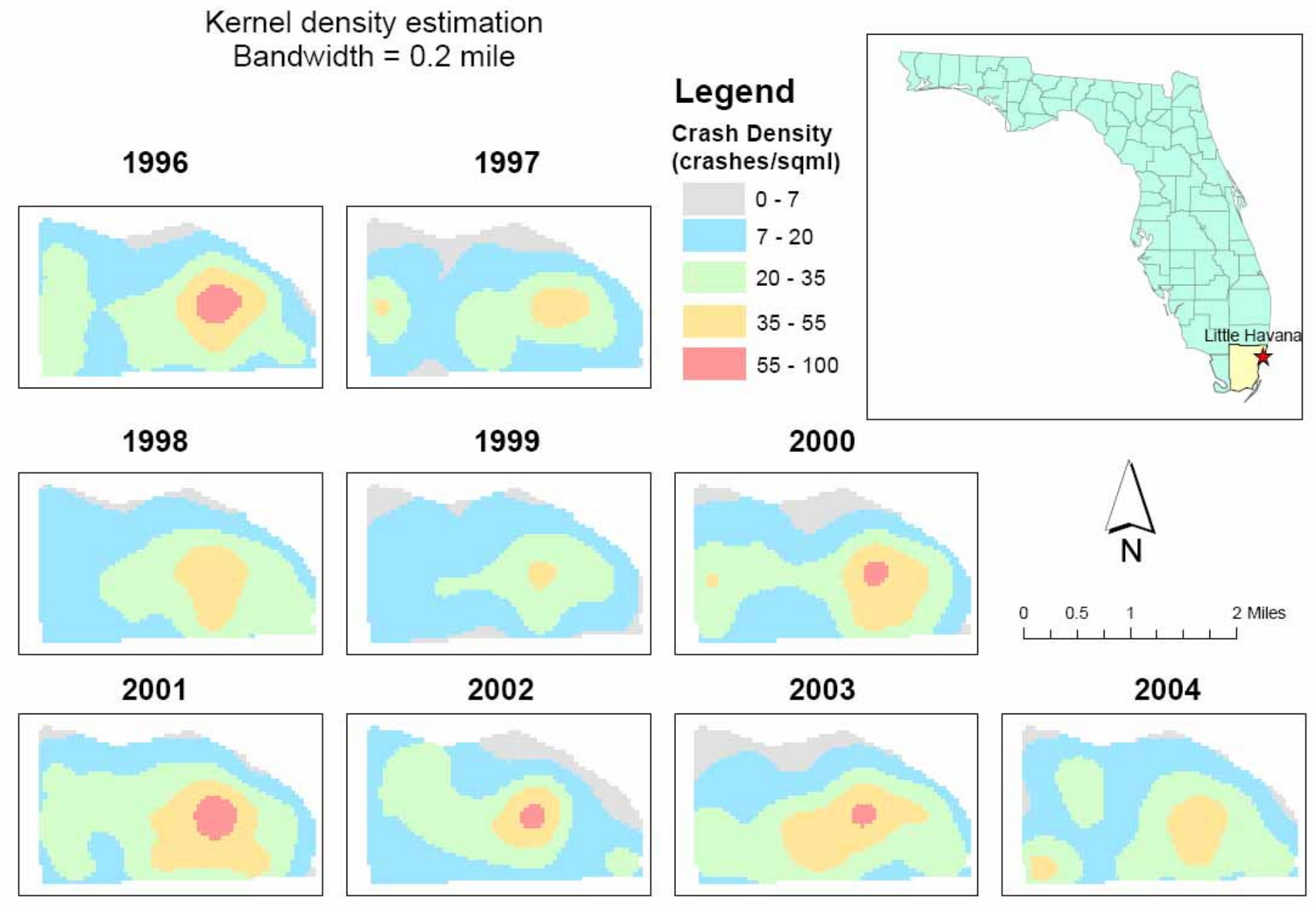

Figure 69. Little Havana Crash Density Map (Kernel density estimation; bandwidth = 0.2 mile). 


\section{South Beach Pedestrian Crash Zone Density Map (1996-2004)}

Kernel density estimation; bandwidth $=0.2$ mile

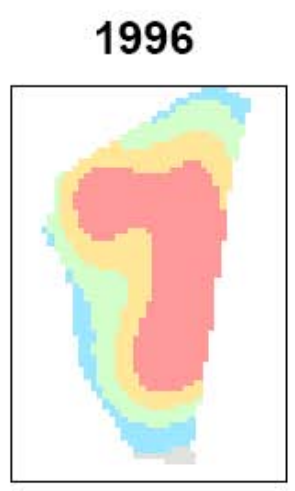

1997
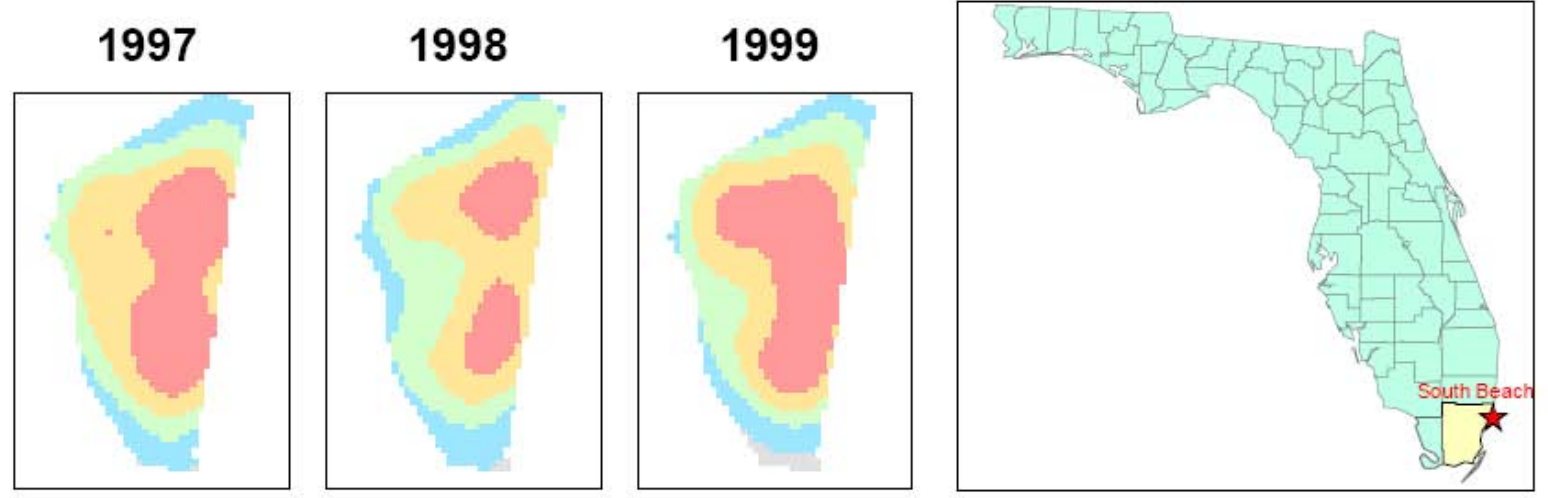

\section{Legend}

2000

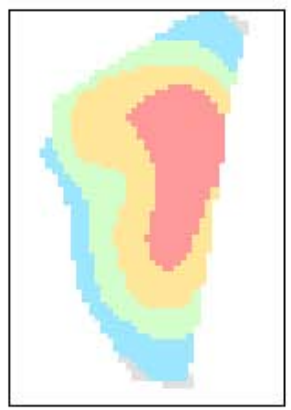

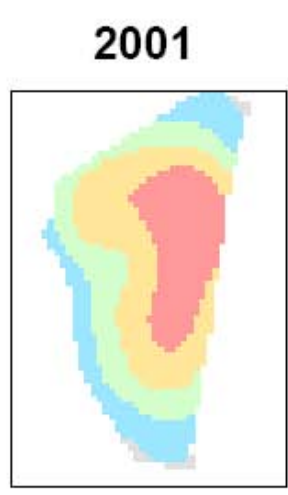

2

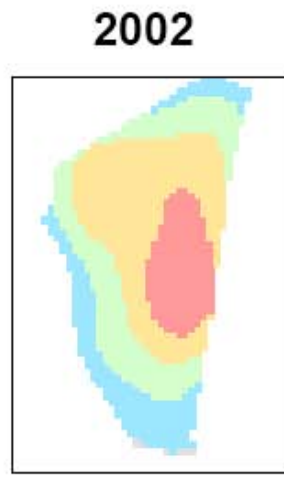

4 Miles
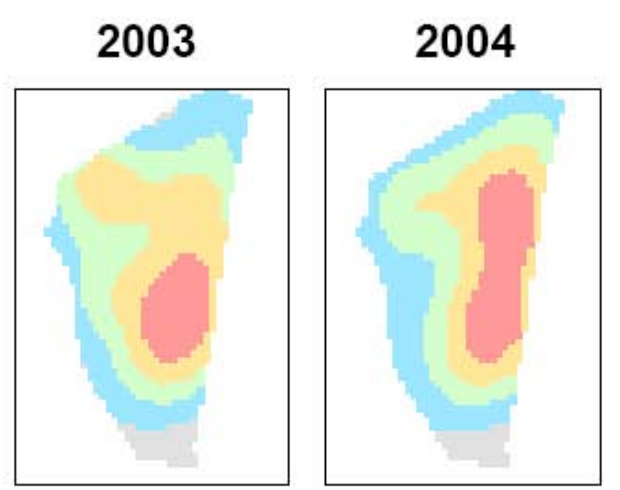

Crash Density (crashes/sqml)

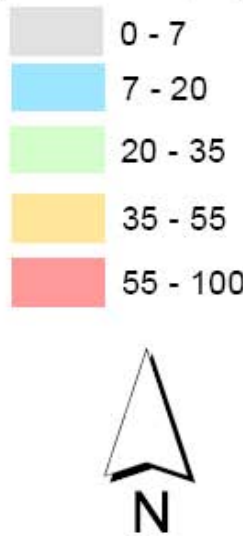

Figure 70. South Beach Crash Density Map (Kernel density estimation; bandwidth $=0.2$ mile). 
In addition to being used to identify high-crash zones in this study, crash densities were also used in the zone-by-zone evaluation of the pedestrian safety program. Specifically, the distribution of the cell-based crash densities was determined before and after program implementation in each of the four zones.

The spatial analysis of crash density can illustrate the spatial pattern of pedestrian crashes across years, as well as generate crash density measures at the land cell/grid level. The generated density measures could further facilitate the administration of a before and after comparison of the frequency distribution of the crash density values. The crash density dataset is cell-based. Each cell contains a value of crash density in the unit of number of crashes per square mile per year. The standardized cell size is $200 \mathrm{ft} * 200 \mathrm{ft}$. Liberty City was divided into 5,817 cells as shown in Figure 71. The numbers of cells in Little Haiti $(2,006)$, Little Havana $(2,172)$, and South Beach $(1,267)$ are also shown in Figure 71.
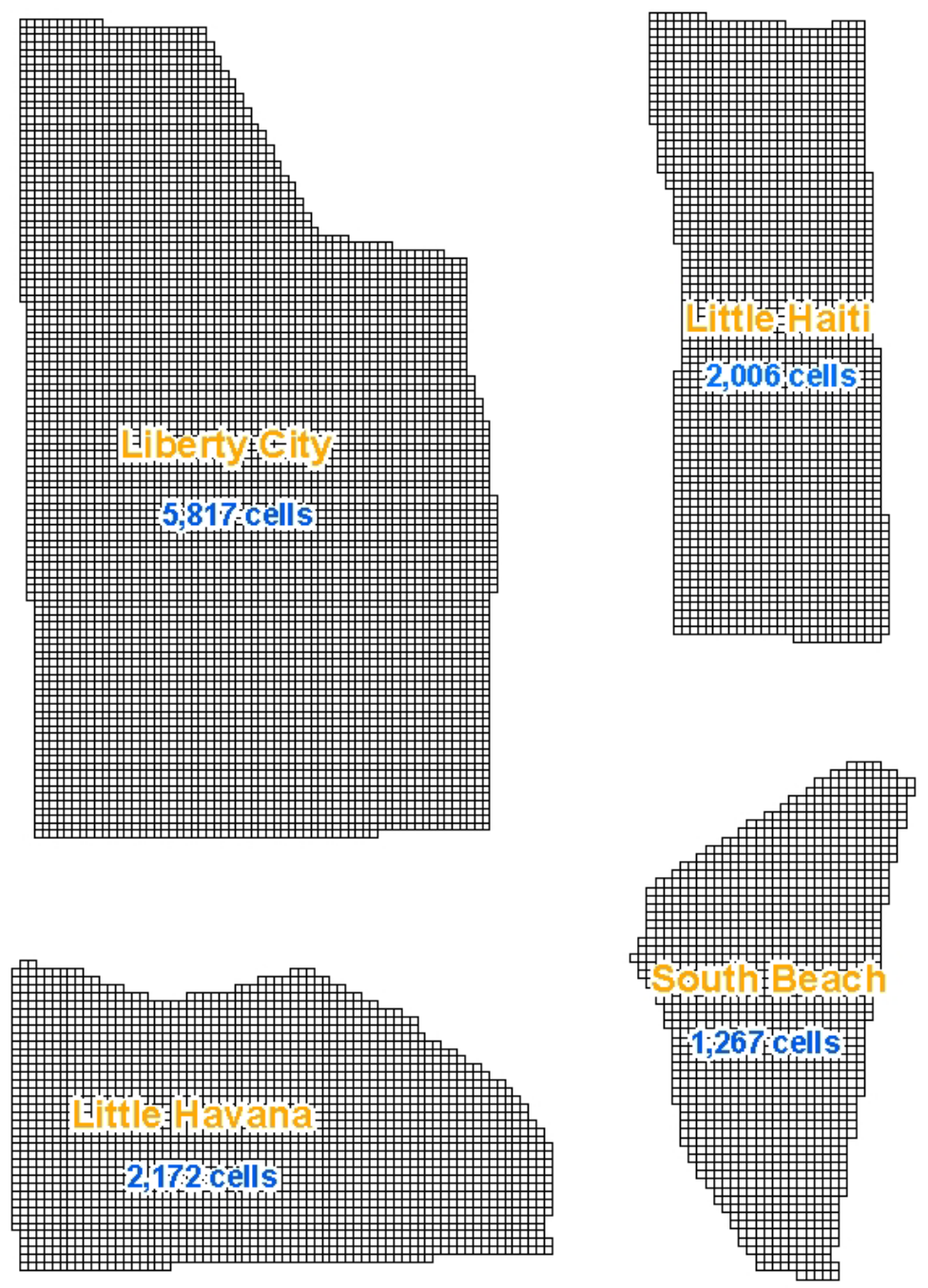

Figure 71. Standardized Cells in the Study Area. 
Given the safety effects of the countermeasure program, changes can be expected in the crash density values in those cells from pre-program period (1996-2001) to post-program period (2002-2004). An ideal change in the frequency distribution of the crash density values would be fewer high-crash cells and more low-crash cells in the post-program period than in the preprogram period. One would expect the distribution to generally shift towards the lower end of the court scale after the countermeasure program, if the program is effective in mitigating the high concentration of pedestrian crashes within the zone.

The following figures display the changes in the frequency distribution of the crash density values from the pre-program period to the post-program period in Liberty City (Figure 72), Little Haiti (Figure 73), Little Havana (Figure 74), and South Beach (Figure 75).

Figure 72 shows that several of the higher values in the pre-program period disappeared after the program, as expected. In Liberty City, the number of extremely high values in median crash density after the program is much less than that of the pre-program period. The mean and standard deviation of the after-period distribution is also smaller than those of the before-period. These results suggest that the countermeasure program is related to a decrease in crash density in a number of cells across the Liberty City zone.

Figure 73 shows a before-after comparison of the crash density values in Little Haiti. Although the mean difference is very small, the standard deviation, or "spread of the data," decreases in the after period. This indicates a less dispersed pattern of pedestrian crash locations after the program in Little Haiti.

Figure 74 shows no clear improvement in the frequency distribution or standard deviation of crash density values in Little Havana. Thus, the changes in the spatial pattern of pedestrian crashes in Little Havana were not appreciable.

Figure 75 shows South Beach's similar pattern to Liberty City (Figure 72). In other words, South Beach experienced a significant decrease in the mean, median, and standard deviation of the crash density from the before-period to the after-period. 


\section{Liberty City Before-After Comparison}

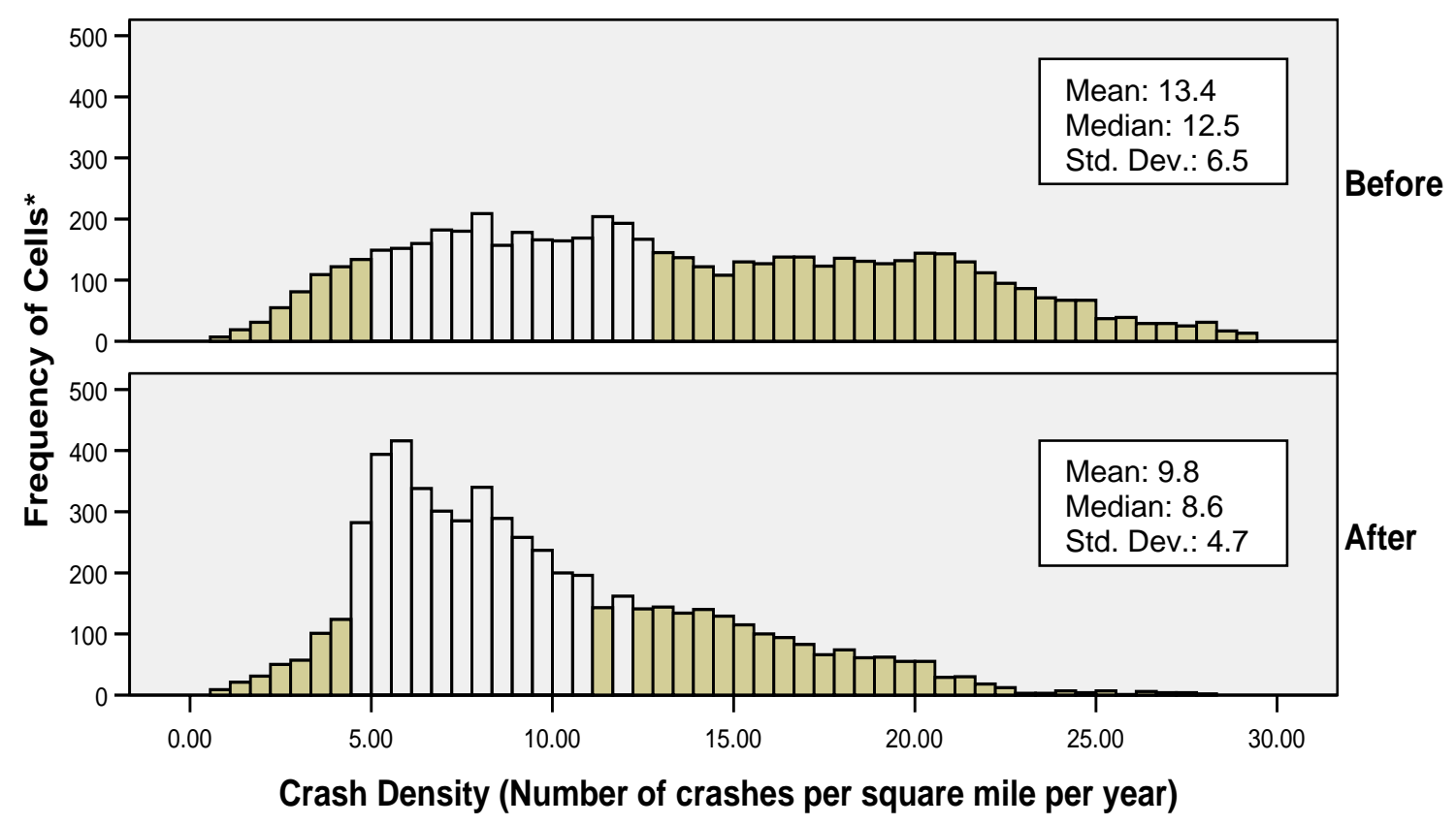

Note: The cell size is $200 \mathrm{ft}$ by $200 \mathrm{ft}$. A total of 5,817 cells are in the crash zone of Liberty City.

Figure 72. Liberty City Before-After Comparison of the Cell-Based Crash Density Values. 
Little Haiti Before-After Comparison

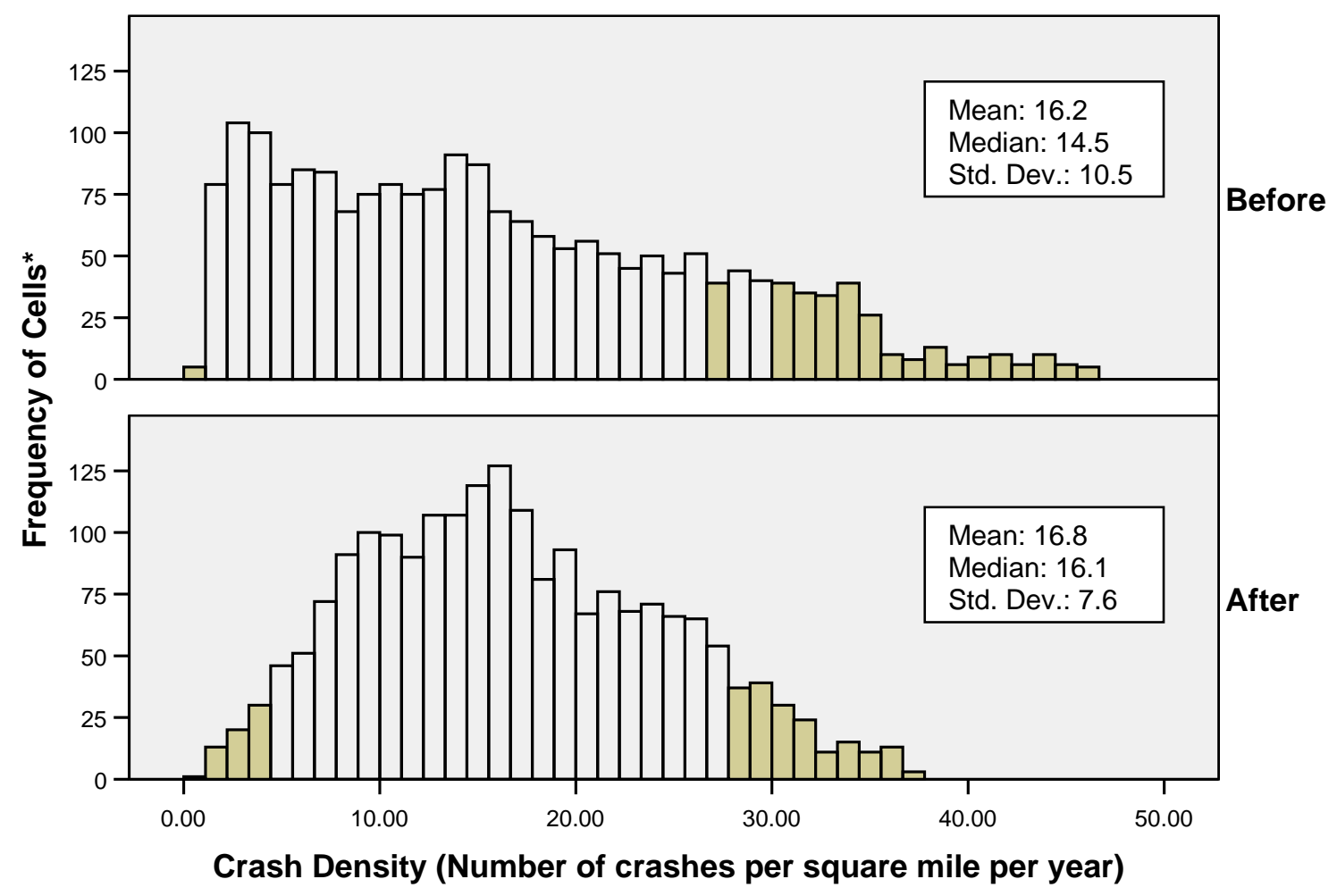

Note: The cell size is $200 \mathrm{ft}$ by $200 \mathrm{ft}$. A total of 2,006 cells are in the crash zone of Little Haiti.

Figure 73. Little Haiti Before-After Comparison of the Cell-Based Crash Density Values. 


\section{Little Havana Before-After Comparison}

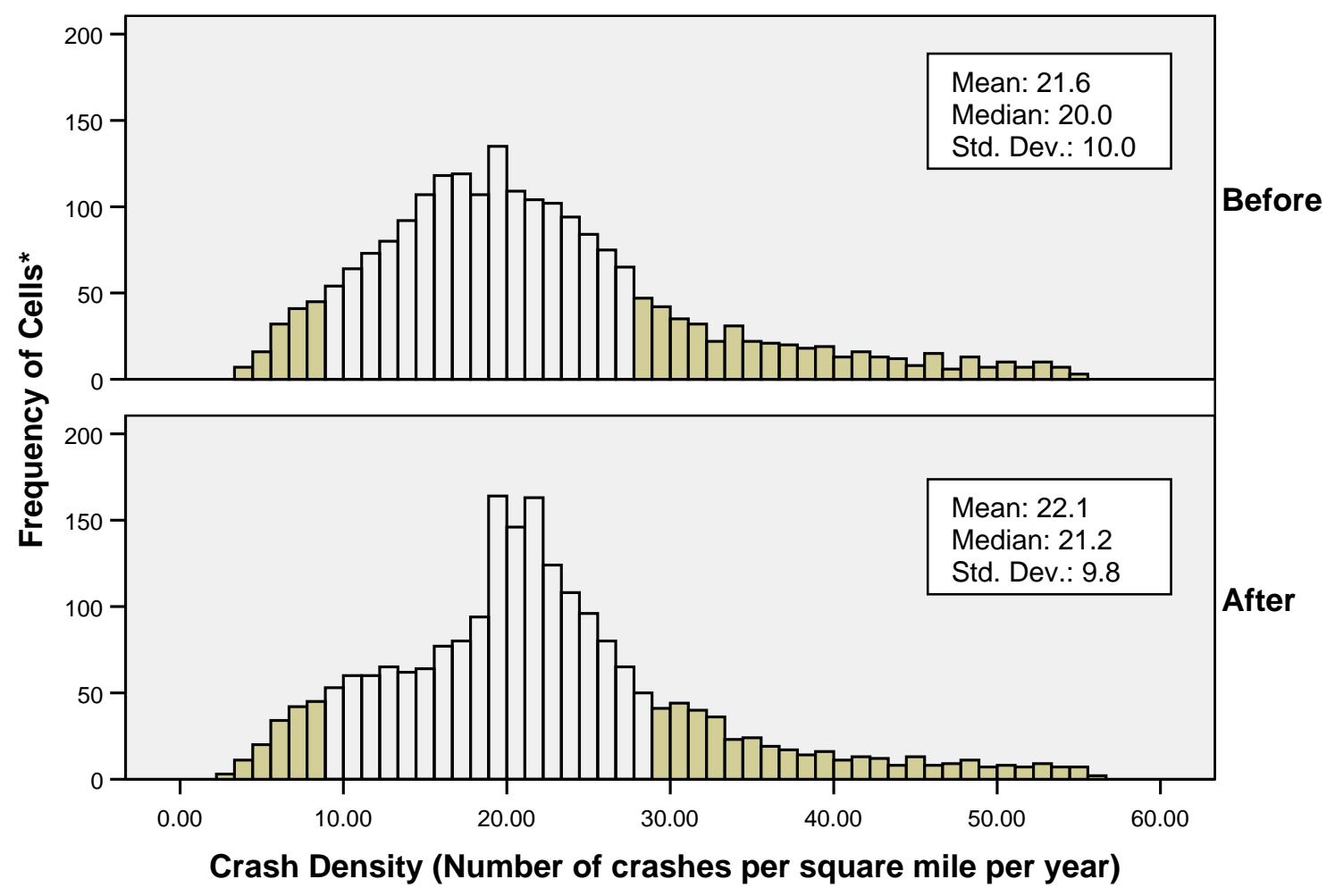

Note: The cell size is $200 \mathrm{ft}$ by $200 \mathrm{ft}$. A total of 2,172 cells are in the crash zone of Little Havana.

Figure 74. Little Havana Before-After Comparison of the Cell-Based Crash Density Values. 


\section{South Beach Before-After Comparison}

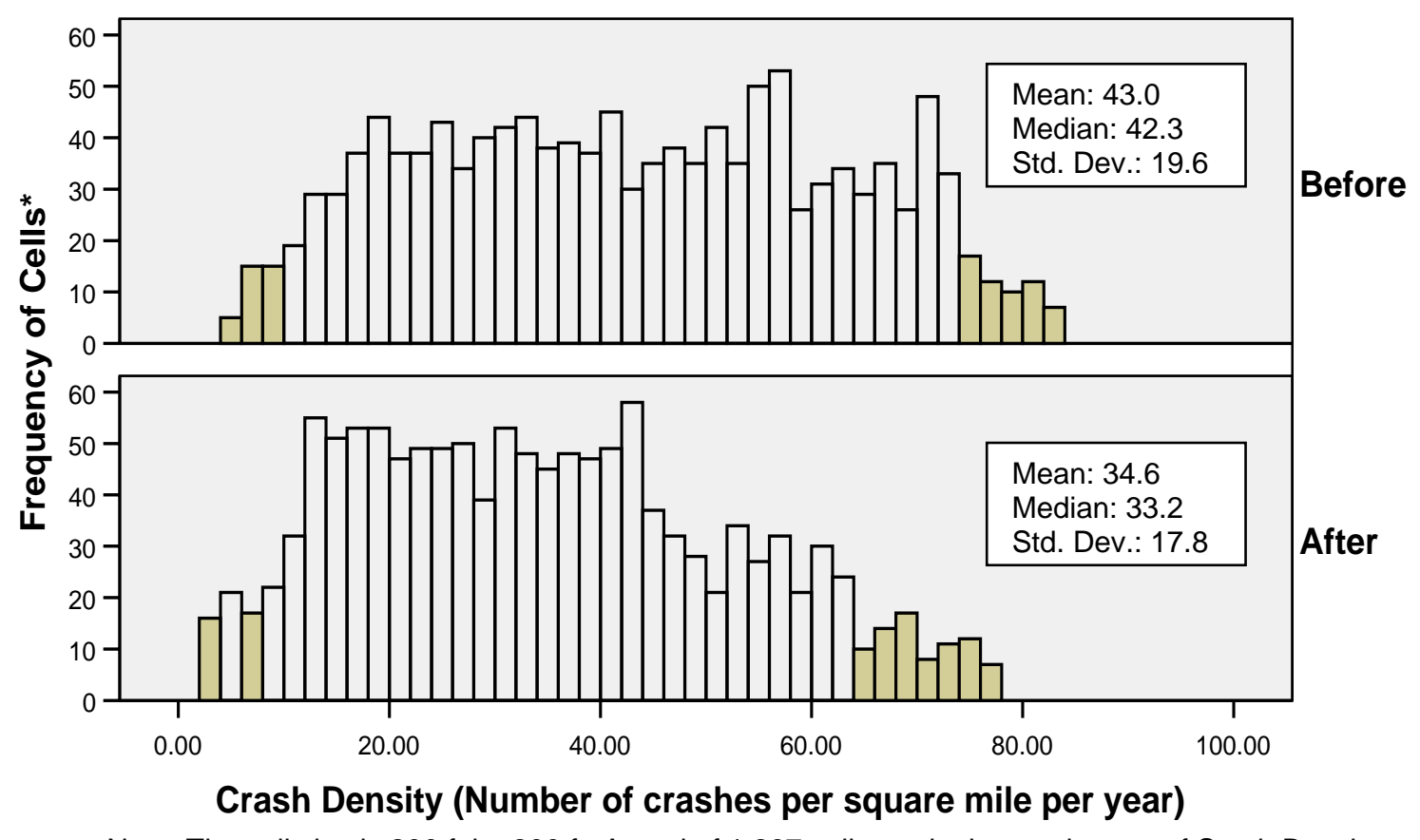

Note: The cell size is $200 \mathrm{ft}$ by $200 \mathrm{ft}$. A total of 1,267 cells are in the crash zone of South Beach.

Figure 75. South Beach Before-After Comparison of the Cell-Based Crash Density Values.

\section{Descriptive Analysis of Crash Frequency}

This section compares crash frequency of targeted sub-populations of the zones by time periods. For each sub-population of interest, the significance of changes in crash frequency is tested from the pre-program period 1996-2001 to the post-program period 2002-2004.

For this analysis, numbers of pedestrian crashes (not crash rates) were used. It was not practical to use comparison zones in other counties, since geocoded data was not readily available from other Florida counties within the project's timeframe. Furthermore, it was not appropriate to use other zones within Miami-Dade County, since some of the countermeasures in the program covered areas throughout the county (e.g., radio and television ads, pedestrian safety educational posters on the buses, isolated engineering treatments implemented throughout the county). Thus, none of the county's zones could realistically be considered as (untreated) control zones.

Since no untreated control sites were available for this analysis, the resulting crash effects are less precise than if acceptable control zones had been available. However, the large area of each zone was considered to help insure reasonable data stability. Furthermore, this analysis was primarily intended to document the trends in pedestrian crashes for the specific pedestrian age and ethnic groups which were the targets of the countermeasures in those zones. Non-parametric tests (e.g., Mann-Whitney-U test) were used for statistical significance testing, since the data are 
not normally distributed. Note that the population data in those four zones across time (1996 to 2004) were not available; thus, it was not possible to turn the raw crash counts into crash rates per population.

Figure 76 displays monthly frequencies for each of the four crash zones over the 1996-to-2004 study period. The exact figures of monthly crash frequencies are shown in Appendix B. Results suggest that pedestrian crash frequency in Liberty City and South Beach decreased over time from 1996 to 2004. The general trends of crash frequency over time in Little Haiti and Little Havana are not clear based on the data shown.

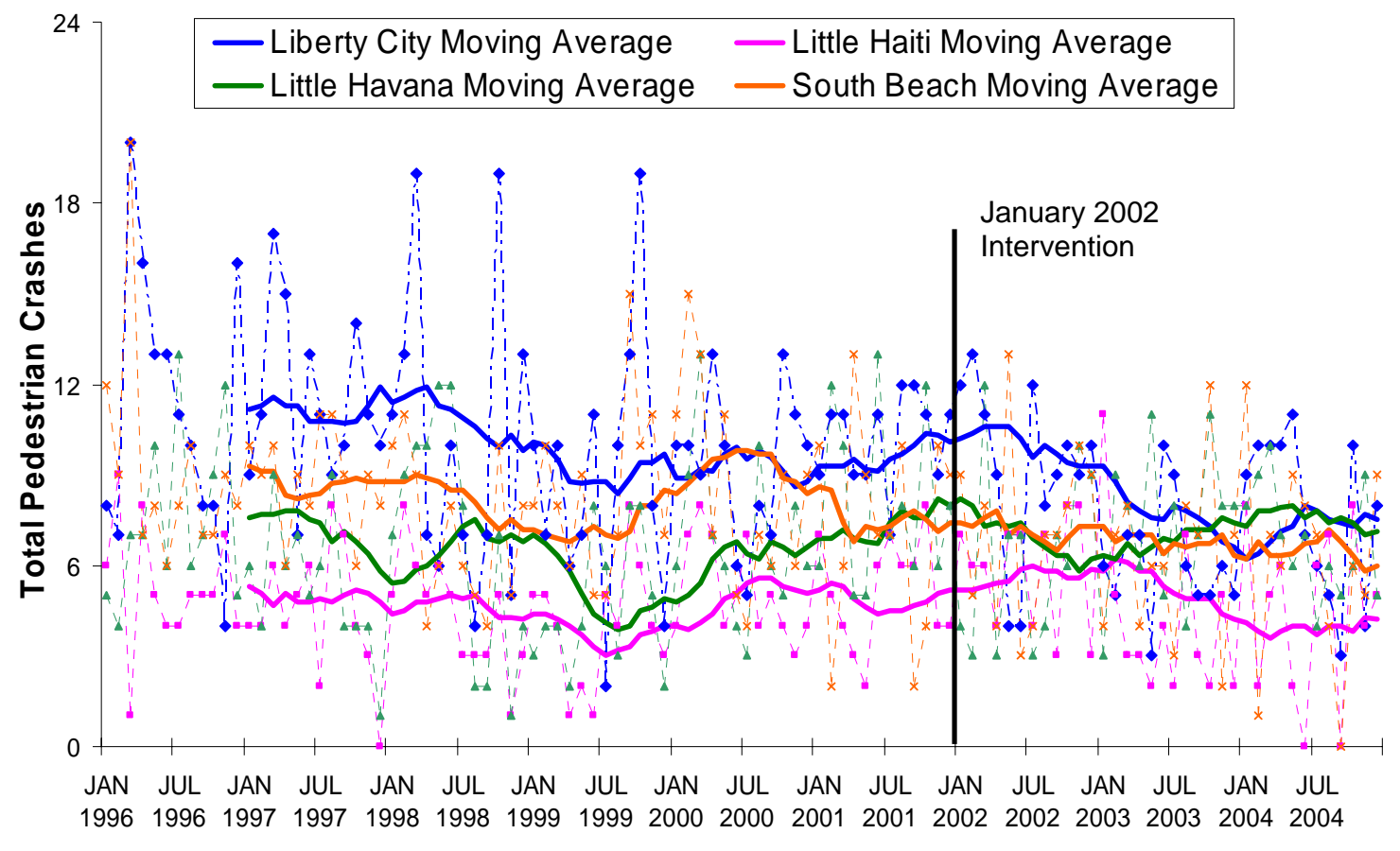

Month-Year

Figure 76. Monthly Pedestrian Crash Frequency by Crash Zones (1996-2004).

Figure 77 shows the pedestrian crash frequency by age groups in Liberty City from 1996 to 2004. A total of four age groups are used, including children (1 to 13), school-age children (5 to 12 ), adults (14 to 64), and older adults (65 and older). Children 5 to 12 years old were considered as a specific study population because most of the pedestrian safety countermeasures were directed at children in elementary schools. The orange line presents the trends of crashes involving school-age child pedestrians, which indicates a slight decrease in number of crashes involving school-age child pedestrians over time. There is also a slightly declining trend in number of crashes involving old pedestrians and adult pedestrians, as shown by the green line and red line respectively. Thus, all age groups within the Liberty City zone exhibited a decline in crashes. 


\section{Liberty City}

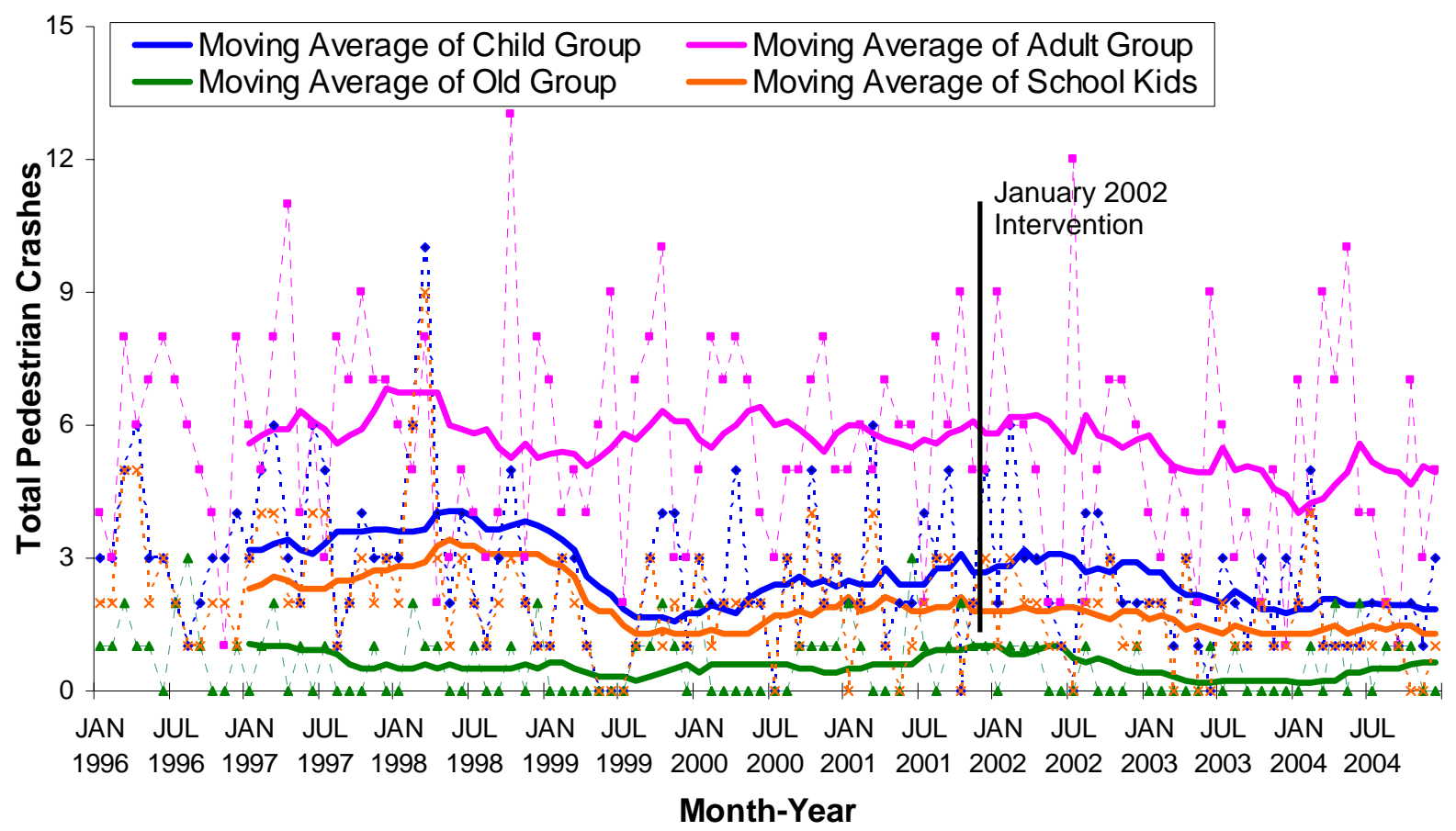

Figure 77. Pedestrian Crash Frequency by Age Groups in Liberty City (1996-2004). 
Figure 78 shows the pedestrian crash frequency by age groups in Little Haiti from 1996 to 2004. None of the four age groups showed clear trends over time.

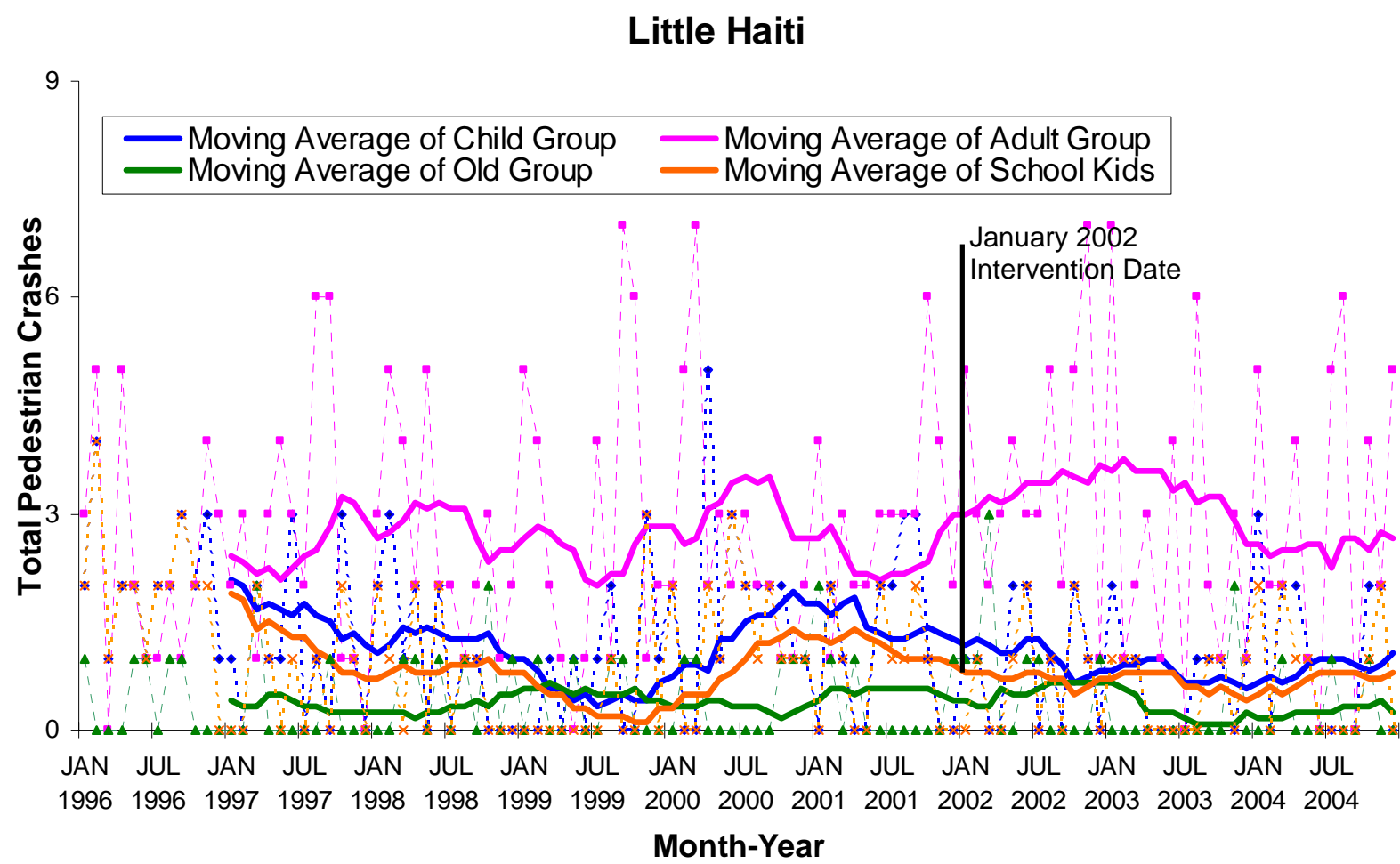

Figure 78. Pedestrian Crash Frequency by Age Groups in Little Haiti (1996-2004). 
Figure 79 shows the pedestrian crash frequency by age groups in Little Havana from 1996 to 2004. Only crashes involving child pedestrians (blue line) or school kids (orange line) appear to have experienced a slight drop from 1996 to 2004. The general trends of the other two age groups are not clear based on Figure 79.

\section{Little Havana}

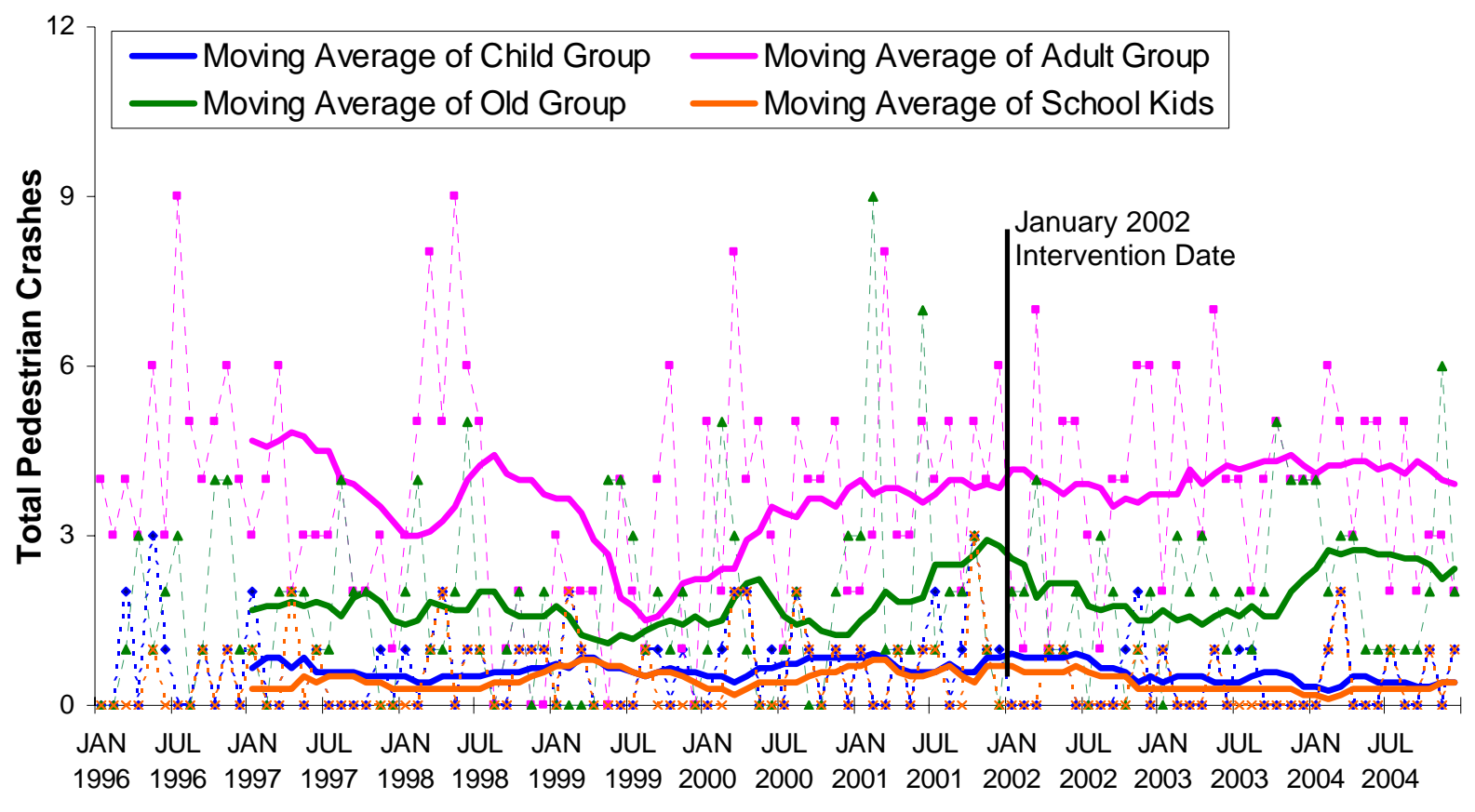

Month-Year

Figure 79. Pedestrian Crash Frequency by Age Groups in Little Havana (1996-2004). 
Figure 80 shows the pedestrian crash frequency by age groups in South Beach from 1996 to 2004. The crash frequency of adult pedestrians appears to have decreased over time. The general trend in the crash frequency of child pedestrians and older pedestrians is not clear.

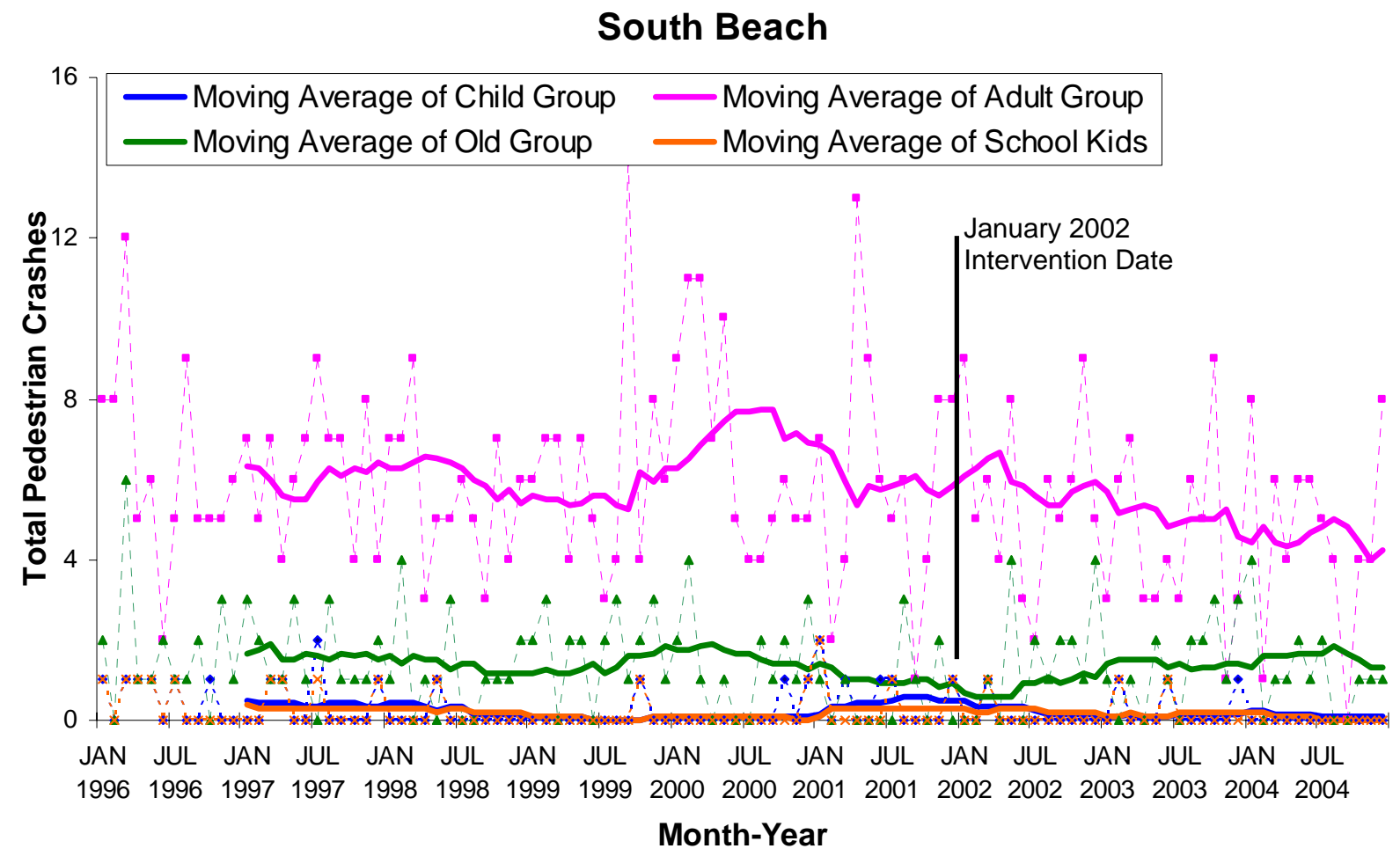

Figure 80. Pedestrian Crash Frequency by Age Groups in South Beach (1996-2004). 
Table 33 presents the average number of pedestrian crashes per month from pre-program period to post-program period by crash zone and age group, along with the mean differences in crash frequency and their significance, calculated using independent $t$ tests. Because the crash counts were not normally distributed (as assumed by the independent $t$ tests), particularly for the analyses stratified by age group, Mann-Whitney-U tests were also conducted. This test is the non-parametric equivalent of independent $t$, but does not require that the outcome be normally distributed. The significance values of the two tests are presented in the last columns of Table 33.

In addition to these non-parametric tests, attempts were made to transform the crash counts to a normal distribution using log-10 and square root transformations according to the Ladder of Powers. However, because the distribution after the transformations did not follow a normal curve well, non-parametric tests were used instead.

Table 33. Mean Difference in Monthly Crashes from Before Period to After Period.

\begin{tabular}{|c|c|c|c|c|c|c|c|c|}
\hline \multirow[b]{2}{*}{ Age Group } & \multirow[b]{2}{*}{ Crash Zone } & \multicolumn{2}{|c|}{$\begin{array}{c}\text { Pre-Program } \\
\text { Period } \\
(1996 / 01-2002 / 01) \\
\end{array}$} & \multicolumn{2}{|c|}{$\begin{array}{c}\text { Post-Program } \\
\text { Period } \\
(2002 / 02-2004 / 12) \\
\end{array}$} & \multirow{2}{*}{$\begin{array}{l}\text { Mean } \\
\text { Diff. }\end{array}$} & \multirow{2}{*}{$\begin{array}{l}\text { T-Test } \\
\text { (p- } \\
\text { value) }\end{array}$} & \multirow{2}{*}{$\begin{array}{c}\text { Mann- } \\
\text { Whitney } \\
\text { U-Test } \\
\text { (p-value) }\end{array}$} \\
\hline & & Mean & Std. Dev. & Mean & Std. Dev. & & & \\
\hline \multirow[t]{4}{*}{ Total } & Liberty City & 10.21 & 3.68 & 7.60 & 2.70 & -2.605 & $0.000 *$ & $0.000 *$ \\
\hline & Little Haiti & 4.77 & 1.95 & 4.71 & 2.55 & -0.053 & 0.905 & 0.798 \\
\hline & $\begin{array}{l}\text { Little } \\
\text { Havana }\end{array}$ & 6.60 & 3.01 & 6.89 & 2.45 & 0.283 & 0.629 & 0.476 \\
\hline & South Beach & 8.29 & 2.97 & 6.46 & 2.80 & -1.831 & $0.003^{*}$ & $0.004 *$ \\
\hline \multirow{4}{*}{$\begin{array}{l}\text { School } \\
\text { Kids } \\
(5-12)\end{array}$} & Liberty City & 2.18 & 1.51 & 1.37 & 0.97 & -0.807 & $0.005^{*}$ & $0.003^{*}$ \\
\hline & Little Haiti & 0.96 & 0.99 & 0.66 & 0.72 & -0.302 & 0.112 & 0.182 \\
\hline & $\begin{array}{l}\text { Little } \\
\text { Havana }\end{array}$ & 0.48 & 0.71 & 0.31 & 0.53 & -0.165 & 0.224 & 0.308 \\
\hline & South Beach & 0.22 & 0.45 & 0.09 & 0.28 & -0.133 & 0.110 & 0.116 \\
\hline \multirow{4}{*}{$\begin{array}{l}\text { Child } \\
\text { Pedestrian } \\
(1-13)\end{array}$} & Liberty City & 2.89 & 1.81 & 2.11 & 1.32 & -0.776 & $0.026^{*}$ & $0.023^{*}$ \\
\hline & Little Haiti & 1.30 & 1.15 & 0.83 & 0.89 & -0.473 & $0.035^{*}$ & $0.047^{*}$ \\
\hline & $\begin{array}{l}\text { Little } \\
\text { Havana }\end{array}$ & 0.68 & 0.80 & 0.43 & 0.61 & -0.256 & 0.096 & 0.125 \\
\hline & South Beach & 0.29 & 0.51 & 0.11 & 0.32 & -0.173 & 0.070 & 0.079 \\
\hline \multirow{4}{*}{$\begin{array}{l}\text { Adult } \\
\text { Pedestrian } \\
(14-64)\end{array}$} & Liberty City & 5.90 & 2.28 & 4.89 & 2.62 & -1.018 & $0.041 *$ & $0.026^{*}$ \\
\hline & Little Haiti & 2.74 & 1.69 & 3.00 & 1.97 & 0.260 & 0.480 & 0.543 \\
\hline & $\begin{array}{l}\text { Little } \\
\text { Havana }\end{array}$ & 3.60 & 2.09 & 3.91 & 1.65 & 0.312 & 0.441 & 0.297 \\
\hline & South Beach & 6.26 & 2.48 & 4.80 & 2.21 & -1.460 & $0.004 *$ & $0.007^{*}$ \\
\hline \multirow{4}{*}{$\begin{array}{l}\text { Old } \\
\text { Pedestrian } \\
(>=65)\end{array}$} & Liberty City & 0.68 & 0.80 & 0.43 & 0.61 & -0.256 & 0.096 & 0.125 \\
\hline & Little Haiti & 0.41 & 0.57 & 0.34 & 0.68 & -0.068 & 0.589 & 0.305 \\
\hline & $\begin{array}{l}\text { Little } \\
\text { Havana }\end{array}$ & 1.73 & 1.71 & 2.11 & 1.41 & 0.388 & 0.246 & 0.080 \\
\hline & South Beach & 1.36 & 1.23 & 1.34 & 1.19 & -0.013 & 0.958 & 0.989 \\
\hline
\end{tabular}

Results show that pedestrian crash frequency in Liberty City and South Beach decreased significantly from pre-program period to post-program period, while Little Haiti and Little Havana showed no significant changes in monthly crash frequency. 
In terms of crashes involving school kids (age 5-12), only Liberty City experienced decreases from pre-program period to post-program period. Liberty City had the largest decrease in the average monthly number of crashes involving school-age child pedestrians, although South Beach had the largest proportional decrease (see Figure 81). The monthly average crash frequency (involving school-age child pedestrians) declined by 0.81 crashes per month $(37 \%)$ in Liberty City, 0.30 crashes per month (31\%) in Little Haiti, 0.16 crashes per month $(34 \%)$ in Little Havana, and 0.13 (60\%) in South Beach. The combined reduction in school kid pedestrian crashes in the four zones was $36.7 \%$.

These results conform to the fact that the child pedestrian safety program was initially and more intensively focused in elementary schools in Liberty City and was later implemented in elementary schools throughout Miami-Dade County. The results are consistent with the effort extended by the Ryder Trauma Center.

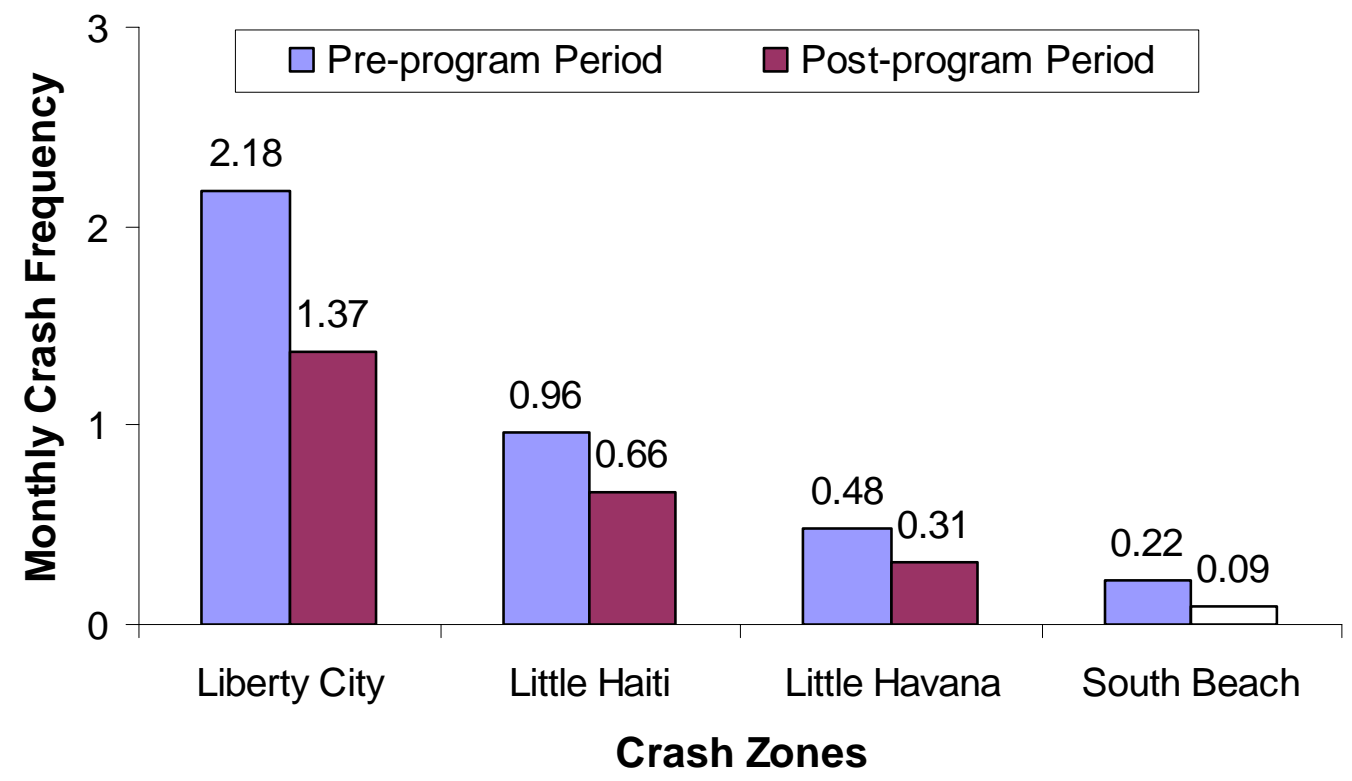

Figure 81. Crash Frequency Involving School Kids (Age 5 to 12) by Zones (1996-2004).

In terms of crashes involving adult pedestrians, no significant changes were found in Little Haiti and Little Havana. However, from pre-program period to post-program period, both Liberty City and South Beach experienced significant drops in the number of adult pedestrian crashes.

Figure 82 illustrates the results. Adult pedestrian crashes declined by $17.1 \%$ in Liberty City, and $23.3 \%$ in South Beach, compared to a slight increase in adult pedestrian crashes in Little Haiti and Little Havana. 


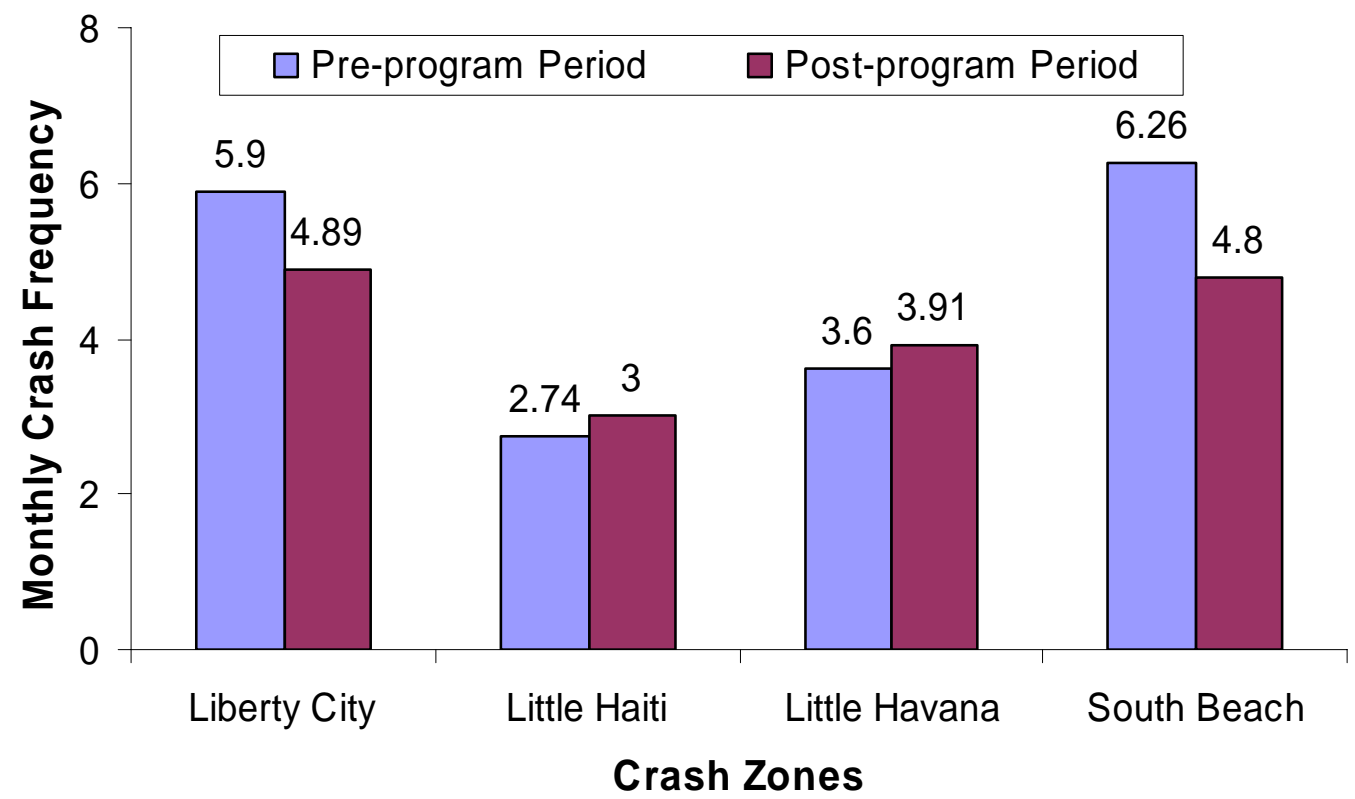

Figure 82. Crash Frequency Involving Adult Pedestrians (Age 14 to 64) by Zones (19962004). 
In terms of crashes involving older adult pedestrians 65 and older, Liberty City is the only crash zone that experienced a significant decline in crash frequency of 0.25 crashes per month (37\%) (significant at $\mathrm{p}<.10$ ). However, there was some suggestion based on the non-parametric results that crashes in Little Havana among older pedestrians actually increased during the post-program period. This may be due to population increases or possibly increases in walking by older adults in these communities (increased exposure). Crash rates per population were not used in these analyses because the population data were not available at the zone level. In any case, the olderadult treatments (mostly safety education materials and radio/TV PSAs) did not appear to result in any measurable benefit to crashes involving older pedestrians (see Figure 83).

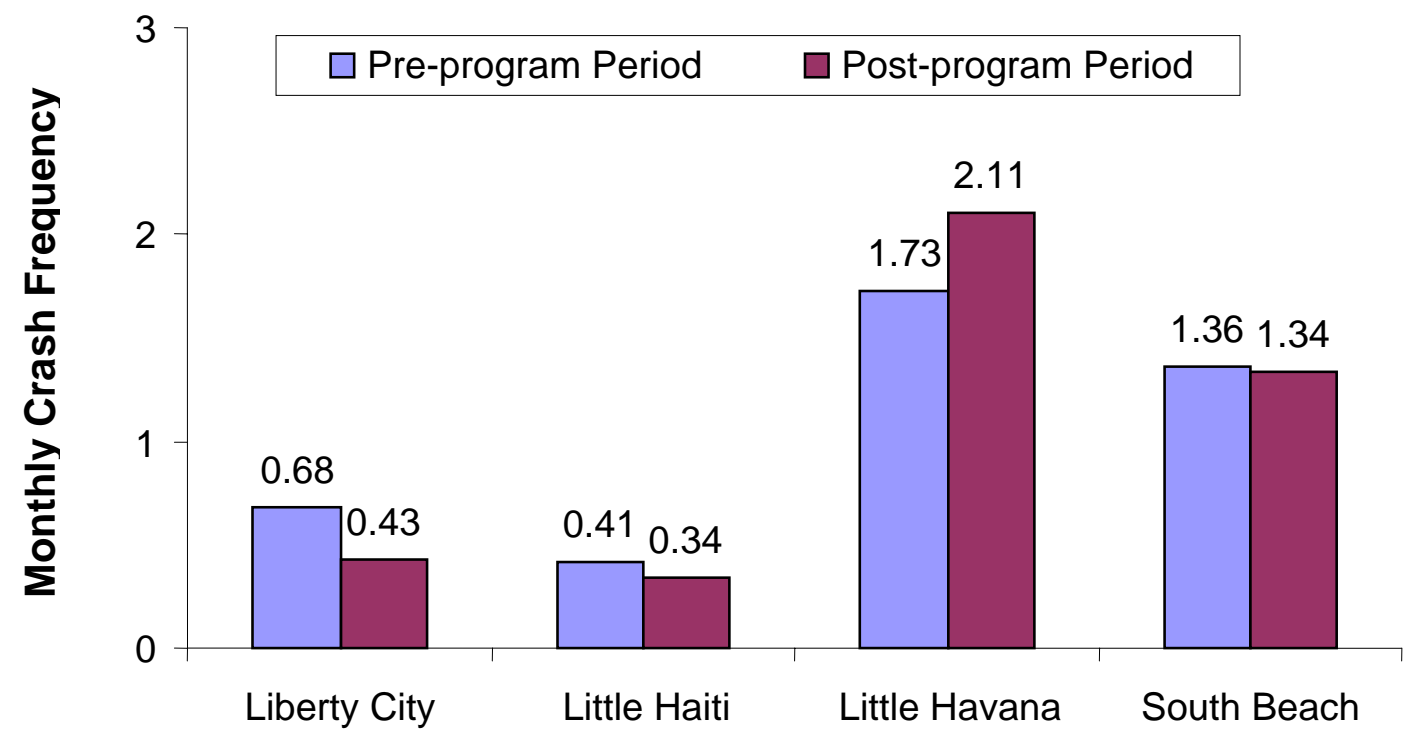

\section{Crash Zones}

Figure 83. Crash Frequency Involving Older Pedestrians (Age 65 and Older) by Zones (1996-2004).

It is encouraging to have observed the clear reduction in the number of pedestrian crashes after program implementation in South Beach and Liberty City. Although no pedestrian exposure data were available on a zone-by-zone basis for use in trying to understand the drop in pedestrian crashes, several points are worth mentioning. First, if the drop in pedestrian crashes were due to some unexplained reduction in pedestrian exposure countywide, one might expect a somewhat consistent reduction in pedestrian crashes in each of the zones, which didn't occur. Secondly, South Beach was the recipient of the most intense infusion of pedestrian safety treatments, compared to the other primary treatment zones. It seems reasonable to assume that this contributed to the largest reduction in pedestrian crashes in South Beach, compared to the other zones. Also, Liberty City received by far the most intense child pedestrian safety program, which may help explain the large reduction in child pedestrian crashes in that zone. 
It is not known why there was no clear reduction in pedestrian crashes in Little Haiti and Little Havana, but there are several possible explanations. First, the most intense countermeasures in Little Havana were educational efforts directed at senior Hispanic pedestrians in senior centers, public buildings, and apartment complexes. In spite of these efforts, there was no measurable effect on crashes among older pedestrians. One possible reason is that it may be more difficult to cause a major shift in the behavior of older pedestrians (i.e., old habits are hard to break) than for younger pedestrians (i.e., children). It may also be in part that it is difficult to reach enough older adults through traditional educational efforts (brochures, TV and radio PSAs) for existing and new older county residents to have a measurable effect. Certainly, further efforts are needed to determine more effective methods to increase the safety of older pedestrians through safety education.

Another possible explanation why Little Haiti and Little Havana may have experienced less pedestrian crash reduction (i.e., for all ages except child pedestrians) is that these two neighborhoods are much less culturally diverse than South Beach and Liberty City, and thus there may be customs and behaviors that may be more resistant to the types of U.S. countermeasures tested in this study. This may be true in spite of the educational countermeasures using Spanish and Haitian Creole messages in addition to English. In short, developing effective pedestrian crash reduction strategies in ethnic neighborhoods may deserve further study.

\section{Crash Types Effects}

Efforts were made to review the various crash types by zone to determine the types of pedestrian crashes that were most affected by the pedestrian safety countermeasures implemented. In general, sample sizes of crash types by zone were not large enough to test for statistical significance. The section below does summarize some of the general trends which were found in the crash type frequencies over the nine-year study period. A more detailed description of this crash type information is given in Appendix D.

\section{$\underline{\text { Liberty City }}$}

The top seven crash types accounted for almost $66 \%$ of the crashes in Liberty City. Dash/dart-out crashes accounted for the largest share of pedestrian crashes. The number of dash/dart-out crashes per year dropped from the before period (14 to 31 crashes per year) to the after period (e.g., 6 per year in 2003). Some reduction was also found in "crossing roadway-vehicle turning" pedestrian crashes, where there was a reduction in the before period (from 9 to 15 per year) to the after period (4 to 6-per year). This is indicative of an increase in a crash type that often occurs among child pedestrians, and may be indicative of the effectiveness of countermeasures used in addressing unsafe behavior among children. There was an unexplained increase in "pedestrian in roadway" crashes in 2003-2004. 


\section{$\underline{\text { Little Haiti }}$}

The top seven crash types accounted for about $62 \%$ of the crashes in Little Haiti. Pedestrians crossing the roadway being struck by vehicles not turning accounted for the large share (more than $16 \%$ ) of collisions in Little Haiti. This corresponds to one of the common practices of pedestrians in that neighborhood who walk to the centerline and wait in the road for vehicles to pass, before they finish crossing. There did appear to be a drop in dash/dart-out crashes and also in the other/unknown grouping. However, there were no clear reductions in other crash types.

\section{Little Havana}

The top seven crash types accounted for more than $75 \%$ of the crashes in Little Havana. Pedestrians were most often struck while crossing the road, with vehicles not turning (18.4\%) or with vehicles turning (17.2\%). Dash/dart-out crashes accounted for $7.5 \%$ of crashes. Crashes increased in some of these crash types over time, and decreased in other crash types, which corresponds to no overall reduction in crashes in Little Havana.

\section{$\underline{\text { South Beach }}$}

In South Beach, the top seven crash types together accounted for nearly $80 \%$ of the crashes, with crossing roadway-vehicle turning accounting for more than $20 \%$. Other predominant crash types included crossing roadway-through vehicle (14\%), unusual circumstances $(13 \%)$, backing vehicle $(12 \%)$, dash/dart-out crashes $(8 \%)$, and off roadway and pedestrians in roadway $(5 \%$ each). The greatest reductions occurred in the backing, and unusual crash types. These results do not point to a clear pattern of pedestrian or motorist behaviors that may have been influenced by the various countermeasures. 


\section{CHAPTER 6: SUMMARY AND CONCLUSIONS}

The purpose of this study was to reduce deaths and injuries to pedestrians in a large urban environment by targeting countermeasures toward specific high-crash locations and zones. Miami-Dade County was selected as the focus of this study because of its large pedestrian crash problem, its age and ethnic diversity, as well as the willingness of State and County officials to participate in the study and elevate pedestrian safety to a high priority. Using pedestrian crash data from 1996-2001, four zones were identified in Miami-Dade County which were found to have high-pedestrian-crash patterns.

This section is focused exclusively on the results of the implementation of the Miami-Dade pedestrian safety program, which was an amalgamation of multiple countermeasures, with some countermeasures more fully developed than others. As mentioned earlier, no attempt was made to isolate the effects of any single intervention in order to evaluate its benefits or to replicate the performance it had achieved in any previous work associated with its development. One overriding objective of this project was to establish a multi-faceted countermeasure program that was operated by the local agencies with only technical and partial financial support from this project. Imposing constraints due to evaluation requirements would likely have dampened any combined effects among countermeasures and created an unrealistic environment. This, in turn, could have deterred the institutionalization of the local pedestrian safety program, which was a second important objective of this project. Given these factors, the results reported herein should not be considered an assessment of any individual countermeasure. In particular, it is not valid to compare performance of any intervention in this implementation to its results when it was the singular focus of an intensive step intervention designed specifically to evaluate the countermeasure's potential.

A total of 16 different pedestrian treatments were targeted to areas within the county, and particularly within the four selected zones (Liberty City, Little Haiti, Little Havana, and South Beach). These countermeasures included education, enforcement, and engineering measures, based primarily on previous NHTSA and FHWA research. The benefits of the pedestrian safety program were evaluated countywide using three years of "after" data (2002-2004).

Two separate types of evaluation were conducted. The first involved using FDOT's computerized crash file for Miami-Dade crashes (1996-2004), termed the DHSMV database. The second analysis made use of a "zone database," which was created by reviewing all pedestrian crash reports and geocoding them by location. The major findings from these two sets of analyses are summarized below.

\section{Countywide Crash Evaluation Results}

For the countywide pedestrian crash evaluation, several control groups were identified. These control groups included Broward County (i.e., the county just north of Miami-Dade County which includes Ft. Lauderdale), six metropolitan counties in Florida (Duval, Hillsborough, Pinellas, Palm Beach, Orange, and Broward counties combined), and all Florida pedestrian crashes (excluding Miami-Dade County). Pedestrian crash rates (pedestrian crashes per 100,000 
population) were also determined for the county and control groups by month and year and used in the evaluations.

It was not considered feasible to determine the effect of each of the individual countermeasures on pedestrian crashes, since several of the treatments had similar or overlapping implementations and target populations. Thus, the overall pedestrian safety program was the focus of the evaluation. Multivariate intervention auto-regressive integrated moving average (ARIMA) time series analysis was used to determine the overall impact of the pedestrian safety programs.

Implementation began for many of the specific pedestrian safety treatments in early 2002, while other treatments were first implemented in late 2002 or in 2003. Accordingly, three different intervention points were tested in each model: January 2002, January 2003, and January 2004. The results of the time-series evaluation revealed the following:

1. The first significant effect of the pedestrian safety program on overall pedestrian crashes was the intervention point on January, 2003. The total effect of the Miami-Dade pedestrian safety program was estimated to be a $13.3 \%$ reduction in pedestrian crashes based on using Broward County as a control series, and an $8.5 \%$ reduction based on using the six metropolitan counties or the statewide crash rates as control series. These reductions were significant at the .05 level. Based on the average post-intervention monthly population in Miami-Dade County, this effect translates into a reduction of about 15 fewer pedestrian crashes per month, or about 180 fewer pedestrian crashes annually in Miami-Dade County.

2. The benefits of the pedestrian safety program continued beyond 2003 in that the average number of pedestrian crashes in 2004 remained lower than the pre-2003 level. However, there was no independent additive reduction detected that could be associated with the pedestrian safety activities conducted during 2004. The ARIMA analyses showed that there was a large reduction in pedestrian crashes in Miami-Dade County during the combined 2003 to 2004 time period after adjusting for other temporal trends (e.g., fuel prices and changes in traffic safety laws) and seasonality using the various comparison series of Florida jurisdictions. The conclusion that this reduction can largely be attributed to the overall pedestrian safety program is supported by the fact that the reductions in Miami-Dade pedestrian crashes were consistently larger than those for other Florida jurisdictions, regardless of how the comparison group was formed.

In summary, pedestrian crashes in Miami-Dade County were reduced by 180 per year for a total of 360 fewer pedestrian crashes during the two year 2003-2004 "after" period. A possible cause of this reduction is the combined pedestrian safety program efforts that began in 2003. The fact that pedestrian crashes per month leveled off during 2004 may indicate that additional countermeasures (or increased countermeasure intensity) are needed to achieve additional reductions in the monthly rate of pedestrian crashes after 2004 , or that additional data points are necessary to be able to detect any additional independent effect of the activities in 2004. 
3. Several of the countermeasures which were part of the overall pedestrian safety program, were directed at reducing crashes among children. One of the primary countermeasures was the "WalkSafe" program, which was a countywide pedestrian safety education program implemented in virtually all of the Miami-Dade County elementary schools. Pedestrian crashes for children (age 1 to 13) and school-age children specifically (age 5 to 12) showed mixed results on a countywide basis. Although Miami-Dade experienced a large decrease in pedestrian crashes among children after January 2003, so did some of the control jurisdictions. Specifically, the analysis results did show a significant reduction in child pedestrian crashes as of January 2003, using Broward County as the control series, which would correspond to an $18.5 \%$ decrease. However, the results of the analysis did not indicate a significant change (.05 level) in the child pedestrian crash rates using the six metropolitan county or statewide control series. This result was clearly affected by the continuing drop in child pedestrian crashes statewide and in the six metro areas, particularly since October 2000. It should be noted that the project team did not have data on exposure to help explain the reduction in child pedestrian crashes in the treated zones or in the control groups. It is possible that a portion of the decrease in child pedestrian crashes may have been the result of less walking among children in the after period, although we have no evidence anecdotal or otherwise to suggest this.

Such gradual but steady reductions in crashes in these two control groups may have been the result of less walking exposure (e.g., fewer children walking to school) and/or the result of statewide pedestrian safety initiatives carried out by FDOT in recent years. It should also be mentioned that the Miami-Dade pedestrian safety education program "WalkSafe" was initiated in the latter part of 2003, and thus the full benefit of the educational program may be expected to occur later than the January 2003 intervention period. More discussion of such an evaluation for the high-crash zones is provided later.

4. The ARIMA analysis of 14- to 64-year-old pedestrian crash rates indicated a significant reduction among this age group in Miami-Dade County starting in January 2003, regardless of the control group used. There was a downward trend in crashes involving this age group in each of the control groups, as well as a steeper downward trend in Miami-Dade County. Using the statewide control series to estimate the magnitude of this effect, the 2003 intervention date was associated with a 0.60 monthly reduction in Miami-Dade 14- to 64-year-old pedestrian crashes per 100,000 population, or about an $8.6 \%$ annual reduction in the average level prior to the pedestrian safety program.

5. The average crash rate for older pedestrians (i.e., pedestrians 65 and older) was lower in Miami-Dade County and also in each of the control groups in the after period compared to the before period. None of the ARIMA models, however, indicated a significant change in the age 65 and older rates in Miami-Dade County at any of the intervention points after controlling for variability using the control series. More discussion on this issue is provided later, particularly with respect to Little Havana, where several countermeasures were directed at older Hispanic pedestrians.

6. The effects of the three-year program were also examined with respect to gender and time of day. These analyses showed mixed results, with generally greater reductions in crashes 
for males and during daylight hours (between 10 a.m. and 6 p.m.) when compared to Broward County crashes. More detailed results are contained in Chapter 5.

Results are summarized in Table 34.

The major premise of the project was that a multi-faceted countermeasure approach might have effectiveness outside of the immediate focal areas and target groups. This certainly has been the case in other studies, e.g., Blomberg et al. (1983). Although it cannot be proven with the available data, it is not unreasonable to assume that the intervention efforts had a county-wide effect. First, many of the countermeasures could not be restricted to the zones, (e.g., bus cards). Second, the population in the county moves around considerably for work and recreation. Thus, it is to be expected that people remote from the focal areas would have been exposed to the interventions. Third, the project team was not aware of any other noteworthy simultaneous pedestrian safety efforts underway. All of this suggests that at least some of the county-wide effect may have been the result of the project's efforts. After all, the safety responsibility of the MPO is not limited to the zones but, rather, covers the entire county. 
Table 34. Summary of Results From the Countywide Analysis.

\begin{tabular}{|c|c|c|c|c|}
\hline \multirow{2}{*}{\multicolumn{2}{|c|}{$\begin{array}{l}\text { Crash Measure } \\
\text { Evaluated }\end{array}$}} & \multicolumn{3}{|c|}{ Effect of Pedestrian Safety Program } \\
\hline & & $\begin{array}{c}\text { Statewide Control } \\
\text { Group }\end{array}$ & $\begin{array}{c}\text { Six Metro Control } \\
\text { Group }\end{array}$ & $\begin{array}{c}\text { Broward Control } \\
\text { Group }\end{array}$ \\
\hline \multicolumn{2}{|c|}{$\begin{array}{l}\text { Total Pedestrian Crash } \\
\text { Rate }\end{array}$} & $8.5 \%$ sig. decrease & $8.5 \%$ sig. decrease & $13.3 \%$ sig. decrease \\
\hline \multicolumn{2}{|c|}{$\begin{array}{l}\text { Child Pedestrian Crashes } \\
\text { (age } 1-13)\end{array}$} & N.S. & N.S. & $18.5 \%$ sig. decrease \\
\hline \multicolumn{2}{|c|}{$\begin{array}{l}\text { School Age Child } \\
\text { Pedestrian Crashes } \\
\text { (age } 5-12)\end{array}$} & N.S. & N.S. & N.S. \\
\hline \multicolumn{2}{|c|}{$\begin{array}{l}\text { Pedestrian Crashes (age } \\
14-64)\end{array}$} & $8.6 \%$ sig. decrease & sig. decrease & sig. decrease \\
\hline \multicolumn{2}{|c|}{$\begin{array}{l}\text { Pedestrian Crashes (age } \\
65+)\end{array}$} & N.S. & N.S. & N.S. \\
\hline \multicolumn{2}{|c|}{ Male Crashes } & $7.3 \%$ sig. decrease & sig. decrease & $9.4 \%$ sig. decrease \\
\hline \multicolumn{2}{|c|}{ Female Crashes } & N.S. & N.S. & N.S. \\
\hline \multirow{5}{*}{$\begin{array}{l}\text { Time of } \\
\text { Day }\end{array}$} & $\begin{array}{l}6 \text { a.m.-10 } \\
\text { a.m. }\end{array}$ & N.S. & N.S. & N.S. \\
\hline & $\begin{array}{l}10 \text { a.m.-2 } \\
\text { p.m. }\end{array}$ & $16.3 \%$ sig. decrease & $16.3 \%$ sig. decrease & $16.3 \%$ sig. decrease \\
\hline & 2 p.m.-6 p.m. & sig. decrease & sig. decrease & sig. decrease \\
\hline & $\begin{array}{l}6 \text { p.m. }-10 \\
\text { p.m. }\end{array}$ & N.S. & N.S. & $13.6 \%$ sig. decrease \\
\hline & $\begin{array}{l}10 \text { p.m.-6 } \\
\text { a.m. }\end{array}$ & N.S. & N.S. & N.S. \\
\hline
\end{tabular}

\section{Zone-by-Zone Crash Evaluation Results}

In addition to the countywide crash analysis, the project team conducted an evaluation of the changes in pedestrian crashes in each of the high-crash zones that were targeted for countermeasure implementation. These high-crash zones were identified after geocoding pedestrian crashes that occurred from January 1, 1996 through December 31, 2001 and plotting approximately 15,472 pedestrian crashes that occurred in Miami-Dade County. These high-crash concentration zones include Liberty City, Little Haiti, Little Havana, and South Beach.

For the zone analysis, numbers of pedestrian crashes (not crash rates) were used. Since no untreated control sites were available for this analysis, the resulting crash effects are less precise than if acceptable control zones had been available. This analysis was primarily intended to document the trends in pedestrian crashes for the specific pedestrian age and ethnic groups which were the targets of the countermeasures in those zones. Non-parametric tests (e.g., MannWhitney-U test) were used for statistical significance testing, since the data were not normally distributed. A summary of the major analysis findings follows: 
1. Pedestrian crash frequency in Liberty City and South Beach decreased significantly for all pedestrian crashes from pre-program period to post-program period, while Little Haiti and Little Havana showed no significant changes in overall monthly crash frequency.

2. In terms of crashes involving child pedestrians (younger than 14 years old), all four crash zones experienced decreases between the pre-program to post-program periods. Liberty City, which had been identified as having the highest concentration of child pedestrian crashes in the pre-treatment period, experienced the greatest absolute reduction in child pedestrian crashes after the pedestrian safety program was implemented. For the four zones combined, there was an overall decrease of child pedestrian crashes from 5.16 per month (62 per year) to 3.48 per month ( 42 per year), a reduction of $32.6 \%$. Countywide the child pedestrian crashes dropped from 23.1 per month (277 year) in the before period to 18 per month (216 per year) in the after period, a reduction of $22.1 \%$. There is no obvious explanation for the drop in child pedestrian crashes in the comparison areas. It is known that Florida has had an active child pedestrian safety program for many years. This may account for the general crash decline. It also could be the result of significantly decreased child pedestrian exposure, although there is no evidence, anecdotal or otherwise, that such a decline occurred. When interpreting the results in Miami-Dade, the similar response in the comparison areas must be considered as well as the possibility that Miami-Dade would not have shown any or as large a crash reduction without the significant countermeasure efforts undertaken.

The child pedestrian safety education program "WalkSafe" was initially implemented at all of the schools in Liberty City and was next implemented in Little Haiti, and then to approximately half of the 200 elementary schools throughout Miami-Dade County. Therefore, one might expect that any effect on reduced child pedestrian crashes would be more pronounced in those zones (i.e., Liberty City and Little Haiti) where the education programs began sooner and were also most intense. In fact, the largest absolute reductions in child pedestrian crashes occurred in these two zones.

3. In terms of crashes involving adult pedestrians (age 14 to 64), no significant changes were found in Little Haiti and Little Havana. From the pre-program to post-program periods, both Liberty City (17.2\% reduction) and South Beach (23.3\% reduction) experienced a significant drop in the number of crashes involving adult pedestrians. The countywide decrease was not statistically significant. The comprehensive pedestrian safety program consisted of a variety of treatments directed at different age groups and ethnic populations. To help to better understand these results, it should be remembered that some of these countermeasures (e.g., posters on transit vehicles) were directed at adult pedestrians in each of these four zones and to a lesser extent, in other parts of the county. South Beach was the zone that received a more extensive amount of pedestrian countermeasures (including being the only zone which received the special police safety enforcement program during the implementation period), which may help to explain why that zone experienced a significant reduction in crashes to pedestrians in the 14- to 64-year-old age group.

4. With respect to older pedestrians (65 and older), there was not a significant decline in crashes in Liberty City, Little Haiti, or South Beach. In Little Havana, there was actually an increase in older pedestrian crashes. These results indicate that the pedestrian safety treatments 
directed at older adults (e.g., mostly safety education material and radio and TV PSAs) did not have the intended effect of reducing crashes involving older pedestrians. The crash results with respect to older pedestrians during the period covered by the data collection were somewhat disappointing. Even though many of the same educational material used by Blomberg and Cleven (1998) in Phoenix were distributed in Miami-Dade, a similarly large crash reduction was not seen. This is not surprising for at least two reasons. First, Blomberg and Cleven (1998) focused exclusively on the older pedestrian and devoted all of their media resources to this audience. This was not the case in Miami-Dade, where pedestrians of all ages were part of the media program. Second, the Blomberg and Cleven study was a much more intensive type of educational effort applied in zones of one square mile radius, which tended to be relatively homogeneous with respect to ethnicity and socio-economics as well as age. The zones in Miami-Dade were far more heterogeneous and significantly larger.

5. Countywide, the largest amount of crash reduction involved adult pedestrians. While it is not known specifically which of the 16 countermeasures may have accounted for this reduction, several of the countermeasures targeted adult pedestrians and drivers. These included the series of educational posters on buses, several of the safety brochures (e.g., "Pedestrian Walk Safely," "You and You Should Never Meet," the pedestrian safety enforcement program, and others.

6. Spatial analysis of pedestrian crashes countywide identified high-crash zones and to also monitor crash intensity levels over the pre-project and post-project periods using ArcView GIS and Crimestat software. The results clearly show a general reduction in the high-crashconcentration (darker color) areas countywide and in the four selected zones. The reduction in high-concentration areas was more pronounced in Liberty City and South Beach. Changes in spatial distribution were much less notable in Little Haiti and Little Havana. This result was confirmed by the distribution of cells by crash frequency, where the mean crashes per cell dropped between the pre-project and post-project periods in Liberty City (from 13.4 to 9.8 crashes per square mile per year) and South Beach (from 43.0 to 34.6). No such change (in mean crash density per cell) occurred in Little Havana and Little Haiti. These results confirm earlier findings about the greater effectiveness of the pedestrian safety program in certain zones.

\section{Results of Additional Projects}

\section{Pedestrian Law Enforcement Countermeasure}

Within the overall Miami-Dade pedestrian safety project, a related effort for enforcement of driver behaviors with respect to pedestrians was conducted. A pedestrian law enforcement countermeasure was implemented in South Beach during 2002. Research by Van Houten and Malenfant (2003) on driver yielding behavior at pedestrian crosswalks found that police enforcement programs targeting motorists who fail to yield to pedestrians in crosswalks resulted in both initial and sustained increases of yield to pedestrian behavior by motorists at intersections where enforcement countermeasures were conducted, compared to the control group of intersection locations. 
More specifically, the study assessed driver yielding behavior at four crosswalks in each of two, an east and west, high crash corridors in the City of Miami Beach. The eight selected crosswalks received enforcement treatments comprised of police teams which included a decoy pedestrian who crossed the street when other pedestrians were not present and a spotter who radioed failure to yield violations to other officers who flagged the violators and gave them a warning or citation and an enforcement flier.

The police stopped 1,562 motorists for failing to yield to pedestrians over the period of a year, with 1,218 of these stopped during the first two weeks of the program. Three hundred seven citations were issued, of which 188 were given during the first eight weeks of the program. At baseline, $3.3 \%$ and $18.2 \%$ of the drivers yielded to pedestrians in the west and east corridors, respectively. The introduction of the enforcement program at the four sites in the west corridor led to an increase in yielding to $27.6 \%$ during the first week of the program while no increase in yielding occurred at the untreated east corridor. The introduction of the enforcement operations in the east corridor led to an increase in yielding to $28.8 \%$ in this corridor while increased yielding was maintained in the west corridor. Monthly follow-up data indicated that the gains produced by the program were maintained in the absence of high levels of police enforcement, with overall yielding rates of $27.8 \%$ in the west corridor and $34.1 \%$ in the east corridor during the follow-up data collection.

\section{Child Pedestrian Safety Educational Program}

Hotz and colleagues (2004) evaluated the WalkSafe pedestrian educational injury prevention program in four elementary schools located in two high-pedestrian-crash-risk urban school districts in Miami-Dade County Florida. Of the four schools (two schools per district), one school from each district was randomly select to receive the WalkSafe program and the other school served as the control. The WalkSafe program comprises educational curriculum specific to Kindergarten through $3^{\text {rd }}$ grade and $4^{\text {th }}$ through $5^{\text {th }}$ grades and includes class discussion, grade appropriate videos, poster contests and simulation exercises using fictitious roads located on school grounds in order to provide pedestrian safety knowledge to participating students. To promote and sustain participation, the program used multiple components that included events, educational materials, newsletters, walking school buses, and contests.

Using a pre-test/post-test and three month follow-up test, the WalkSafe program was shown to improve the pedestrian safety knowledge of the children participating in the two intervention schools, while no statistically significant difference was detected between the pre-test and posttest scores of two control schools (Hotz et al. 2004). The WalkSafe program incorporated similar messages as NHTSA's "Willy Whistle" program, expanding it in some situations. Although the study by Hotz and colleagues did not assess the individual impact of the various parts of the curriculum, they concluded that the WalkSafe pedestrian educational program was shown to improve knowledge of pedestrian safety among elementary school children. The study did not evaluate changes in pedestrian behavior. 


\section{Study Limitations}

\section{Crash Data}

All pedestrian crashes for Miami-Dade County were reviewed by hand to determine crash type, geo-coded location, and to assure that the crash location was within the county. Note that such careful hand sorting was not possible for pedestrian crashes within the control sites (e.g., statewide, Broward County, and six metro areas). Thus, all statistical analysis involving control sites had to make use of computer-coded crash records for purposes of consistency.

Each dataset used for the analyses herein was subject to some minor and normal biases and potential inaccuracies. The counts of crashes from the DHSMV computer files clearly contained some miscodes that were likely the result of keying errors or problems in reading the entries on the PCR when entering the data. The zone and Miami-Dade files have these errors due to miscoding removed but may understate the actual number of crashes to the extent that MiamiDade pedestrian crashes are miscoded either as non-pedestrian events or attributed to other counties.

None of these problems is considered to have affected the analyses conducted. First, the inaccuracies in the DHSMV data are most likely random rather than systematic. Second, the number of Miami-Dade crashes miscoded into other counties is likely small. Miami-Dade has the largest number of crashes in the State by far. Thus, the staff members doing key entry are likely accustomed to entering a county code of " 1 " for Miami-Dade. This makes it more likely that crashes from other counties will be miscoded as Miami-Dade than vice versa. Simply, the coders and keyers might just get in a pattern of entering " 1 " for county.

Given these considerations, it is reasonable to conclude that the biases inherent in the crash datasets did not influence the conclusions presented later in the report (see Chapter 5). If anything, the geocoding process improved in the later years of the analysis (particularly 20022004), and this likely resulted in including a higher percentage of geocoded crashes in the after analysis, which may have resulted in slightly "understating the beneficial effects of the safety program (i.e., missing more of the geocoded pedestrian crashes in the before period than the after period), although this likely had a small effect on the magnitude of the effects demonstrated.

\section{Behavioral Data}

The design for the Miami-Dade pedestrian demonstration included the use of pedestrian crashes as the sole outcome measure of effectiveness. While intermediate measures of pedestrian and driver behavior have been used in other evaluation studies of pedestrian countermeasures, they were not feasible in the current effort.

\section{Exposure Data}

Exposure data refers to the amount of walking by pedestrians by age group within each zone or areas in the Miami-Dade County, as well as in the control groups. Such data were not available. Lack of exposure data among various age groups and zones did not allow for a full 
understanding of the trends in pedestrian crashes, such as the drop in child pedestrian crashes in the Miami-Dade zones and the statewide control groups. These data are difficult to collect, but would be an asset to future studies.

\section{Countermeasure Intensity}

As stated earlier, the Miami-Dade project was first and foremost a naturalistic implementation of a local pedestrian safety program based on researched countermeasures. The project evaluation was intended to examine as many program effects as possible without interfering with the "normal" process of city, county, and private groups mounting interventions. Unfortunately, it is not typical for non-research safety organizations to collect detailed process information. Thus, for example, even though the total number of brochures printed may be known, the total actually distributed would not be tallied. As a result, there is less than complete insight into the intensity of each intervention. Where quantitative process numbers are known, they are presented in this report. Otherwise, qualitative estimates are given if there was sufficient anecdotal evidence on which they could be based.

\section{Analysis Period}

We had to cut off the after period at the end of 2004 due to contractual scheduling for the NHTSA project. It would be insightful to look at future years (2005 and 2006) to determine the continuing trends in pedestrian crashes, particularly with the addition of engineering treatments in 2005 and 2006. However, this was outside of the scope of this project.

\section{Countermeasure Selection and Evaluation}

The pedestrian demonstration program in Miami-Dade was intended from the outset to be an amalgam of tested and proven countermeasures from NHTSA and FHWA research together with innovative intervention approaches. No attempt was made to be able to discern individual countermeasure effectiveness. The basic premise of the project was to focus as many proved countermeasures on the problem as possible with crashes as the only real measure of effectiveness. Project resources did not permit detailed countermeasure implementation related to pedestrian crashes involving alcohol use, although heavy alcohol use was recognized as a concern in Miami-Dade County, especially in South Beach. While many other possible treatments were available and considered, the ones that were chosen were selected based on specific identified problems and appropriate methods to reduce those problems, with input from the study team. It would be inappropriate to suggest that other jurisdictions follow precisely what Miami-Dade did. We would propose that the next generation of pedestrian safety program examine both the successes and the failures in Miami-Dade and take from them those lessons learned that they believe are applicable to their own environment.

This approach has the significant strength of combining successful interventions under the reasonable theory that there will be synergy among them. Unfortunately, this strength comes at the expense of being able to assess the impact of each individual intervention. Most of the successful countermeasures were tested in isolation and as essentially a step intervention. This facilitated the ability to examine their effects during the developmental efforts. In Miami-Dade, 
all interventions were permitted to ramp up at whatever pace the cooperating implementer chose. Since there was no way to disentangle individual effects, and there was no clear intervention point, the Miami-Dade results reported herein should not be considered a replication of any of the studies associated with countermeasure development and initial testing.

\section{Discussion and Lessons Learned}

\section{Study Results}

The results of these analyses reveal that the combined Miami-Dade pedestrian safety program was associated with a significant reduction in pedestrian crashes countywide, and particularly among child pedestrians within certain focus zones. The analysis revealed that Miami-Dade experienced approximately 180 fewer pedestrian crashes per year in the first two years (2003 and 2004) post-treatment, as a result of the program implementation. The most dramatic effect of the pedestrian safety program occurred in 2003, when most of the countermeasures were in effect, and similar crash reductions continued through 2004. To sustain such a reduction in pedestrian crashes, there is a need to maintain all of the countermeasures at "full strength" in subsequent years of the program in order to sustain the pedestrian crash reduction.

In addition, the process of targeting countermeasures to specific age and ethnic groups appears to have been particularly successful in Liberty City and South Beach. Liberty City was the zone which received the most intense pedestrian safety education programs in all of its elementary schools, and child pedestrian crashes experienced greater absolute crash reductions compared to other zones and proportionally higher than countywide. Of interest is the fact that after the pedestrian safety program, child pedestrian crashes decreased by $32.6 \%$ in the four targeted zones, and decreased by $22.1 \%$ countywide. Based on crash reductions in 2003 and 2004 combined, these reductions agree closely with the reduction of approximately 20 to $30 \%$ in child pedestrian crashes due to "Willy Whistle" and "And Keep on Looking" educational programs conducted in cities with comparable size and evaluated by NHTSA in the 1980s and 1990s.

Of the four zones targeted for specific countermeasures, South Beach was the recipient of the most intense amount of countermeasures, including selective police enforcement, a variety of educational and media messages, as well as a few engineering treatments. It is, therefore, encouraging that South Beach was found to be associated with a substantial reduction in pedestrian crashes $(22 \%)$ along with a $25.5 \%$ reduction in Liberty City.

Not all of the countermeasures were successful, however, in reducing targeted crash types in all of the identified high-crash zones. Most notably, a variety of educational countermeasures (in English and Spanish) were implemented in Little Havana, where there had been a high prevalence of crashes involving older, Spanish-speaking pedestrians. Countermeasures included the distribution of educational material at senior centers, safety educational meetings, television and radio messages, and other educational measures. In spite of these efforts, there was no significant reduction of crashes involving older pedestrians or involving pedestrians in general in Little Havana as a result of the countermeasures implemented there. Likewise, no significant reductions in pedestrian crashes resulted in Little Haiti as a result of the safety program. Such results may provide some understanding about what might be expected from similar pedestrian 
safety programs, and perhaps how to address more challenging crash problems, such as crashes involving older pedestrians.

Conversations in 2007 with trauma doctors at the Ryder Trauma Center (Jackson Memorial Hospital, University of Miami Medical School) have revealed that they have activities underway to begin an aggressive program to reduce pedestrian crashes involving older pedestrians. The Ryder Trauma Center has recently obtained detailed pedestrian crash data from the MPO Pedestrian Coordinator for use in that effort. The goal of this new project is to identify specific areas where the greatest crash problems occur to older pedestrians and then to apply a targeted array of countermeasures. Evaluation of that program is also part of the study goals.

Of the 16 countermeasures that were part of the pedestrian safety program, only a few of them dealt with the nighttime visibility problems. For example, educational posters were placed throughout the county at public places. These posters explain the reduced visibility of pedestrians at night and the need for drivers to slow down at night and for pedestrians to realize that drivers cannot see them well without their use of retro-reflective clothing, a flashlight, etc. There were also engineering recommendations to install overhead lighting at the intersections along certain corridors, such as in South Beach area, where there is a considerable amount of nighttime pedestrian activity and many nighttime pedestrian crashes each year. However, this improved lighting had not been added prior to the end of the after evaluation period (2004).

The implementation period for this study (2002-2004) included a fairly limited number of engineering treatments. Dozens of additional engineering treatments were scheduled to be implemented as part of an FHWA study in 2005-2007. It is expected that the implementation of these additional engineering measures, plus continued targeting of other educational and enforcement countermeasures would help to maintain or even increase the amount of crash reduction countywide and in targeted zones.

This study had the basic objectives of reducing pedestrian crashes in Miami-Dade County and institutionalizing pedestrian safety within the county's political structure. Both of these were somewhat ambitious given the extent of seed money provided by the cooperative agreement and the numerous competing highway safety and national security needs, e.g., seat belts, drunk driving, terrorism. Nevertheless, the results presented herein indicate clearly that these objectives were achieved to some degree. Crashes in Miami-Dade were reduced during the evaluation period, and the data suggest they diminished more than did crashes in reasonable comparison areas. A pedestrian safety program was established within the Miami-Dade MPO and presently continues with significant local support.

Given these positive outcomes, it is reasonable to conclude that a basic approach in which the Federal government provides seed money to establish a local pedestrian safety program is reasonable. It is likely that the total amount of money offered to any jurisdiction should be larger than was available to Miami-Dade from the total amount of the cooperative agreement. The fact that the present effort was overly ambitious, however, does not negate the clear benefits of the approach. Locally mounted pedestrian safety efforts have a high likelihood of focusing countermeasures on the actual problems being experienced. The involvement of local people 
gives a community "ownership" of its pedestrian safety effort and likely promotes greater involvement in the safety effort.

Finally, it must be stressed again that this project was not a research and development undertaking. The research component was the evaluation of an integrated and multi-faceted countermeasure program. A demonstration of this type is a natural culmination of the types of research NHTSA has been committed to since the late 1960s. Developed and tested countermeasures must be adopted by local communities and fully implemented in order to be widely effective. The Miami-Dade project showed that this process is possible and productive. Future efforts should focus on expanding the use of promising countermeasures and learning from the experience in Miami-Dade.

\section{Importance of GIS Data for Program Development}

The importance of having a geographic information system of traffic crash data for the success of this project cannot be overstated. In addition to identifying problem locations and subpopulations, quantifying the problems and evaluating the results, the data are an effective tool for communicating the issues of pedestrian safety and enlisting the support of relevant agencies. The final product, the GIS database of over 15,000 traffic crashes involving pedestrians over nine years, is an extremely powerful tool, which has been very effective in leveraging other pedestrian safety improvements through partners in the FDOT, Miami-Dade County Public Works, MiamiDade Public School Board, and others.

Improving the availability and quality of this type of pedestrian crash data would make it easier to continue implementing and evaluating this type of safety program. Steps that could be taken to improve the safety planning process include electronic crash reporting, the use of GPS to collect location data and more timely release of data by DHSMV. In the interim, making written crash reports available on-line, including the crash location in the DHSMV crash database, and training officers to use a consistent system of identifying crash locations would speed up the current system for mapping crashes.

\section{Interagency Relationships}

Many agencies play a role in improving pedestrian safety so building relationships with police, engineering, health care, funding and educational organizations is essential. Understanding the needs of and opportunities within each group is important because this will help identify the issues that will motivate them to action. Although there may be opportunities for the interests of these different groups to overlap, individual projects are usually executed under the authority of a single agency. Connecting different agencies through their common interest in pedestrian safety was essential to enable the projects to develop.

Specifically, FDOT was an important partner throughout the eight-year project period, and contributed an estimated $\$ 500,000$ toward advancing the geocoding capabilities of the county and in funding a wide range of pedestrian safety education activities and material. This support from Florida was considered to be a crucial ingredient to the overall success of the pedestrian safety effort in Miami-Dade County. In addition, the commitment and resources provided by the 
Miami-Dade MPO and the multiple partners within the county were also essential for the program success. The MPO's strong commitment to pedestrian safety also created a supportive environment for related activities, such as the Ryder Trauma Center's child pedestrian safety activities. Partly as a result of this project, the Miami-Dade MPO now has stronger ties with local police, educators, local and State engineers, hospital staff, and with organizations related to improving pedestrian safety.

\section{Role of Coordinating Agency}

The role of the MPO in this project was to support and coordinate different pedestrian safety activities, not to act as an ad hoc leader of a short-term effort. Supporting the efforts of other groups was seen as a way to increase buy-in from other agencies and to leverage additional resources to the area of pedestrian safety. The most important tools in this regard were the pedestrian crash data and education material provided through the project. The pedestrian crash data were made available to other agencies to make them more keenly aware of the pedestrian safety problem in the area under their jurisdiction and to support their efforts to develop and implement countermeasures. For example, meetings with the City of Miami Beach Police Department early in the project showed that the Department did not use the crash report data that it was generating to track or analyze traffic crashes involving pedestrians. Seeing how crash data were used by the MPO to show the severity of the pedestrian safety problem in Miami Beach helped motivate the MBPD to host the pedestrian safety enforcement training and give greater emphasis to pedestrian safety in its traffic enforcement program. Later contact with the MBPD showed that they had developed their own traffic crash tracking system which gave officers access to current data and led to more targeted enforcement, including pedestrian safety.

Another example is the Miami-Dade County Public Schools' WalkSafe program that was developed by the University of Miami's Ryder Trauma Center. Pedestrian crash maps were used to identify areas in the Liberty City area with large numbers of juvenile pedestrian crashes. Brochures and retro-reflective wrist bands were used to support classroom instruction on pedestrian safety. In the after period, crash data were used again to measure the effectiveness of the WalkSafe program in the Liberty City area.

The coordinating agency should also serve as a pedestrian safety expert by identifying countermeasures and encouraging their implementation by the appropriate agency. The MPO was partially successful in this area through the development of safety educational material in Haitian Creole to serve this at-risk population. The implementation of countermeasures targeting drinking pedestrians was an area that the MPO was not successful. In future projects of this type, staff should ensure that they have a broad knowledge of countermeasure programs that have been developed in other jurisdictions so that the greatest range of options is available.

\section{Institutionalization}

It is believed that one of the greatest benefits of this NHTSA Pedestrian Safety Demonstration study was not just the measurable reduction in pedestrian crashes that occurred, but also the level of institutionalization of a comprehensive pedestrian safety program in Miami-Dade County. The institutionalization of this program by the Miami-Dade MPO Pedestrian and Bicycle Program 
included creating a range of partners, including the Ryder Trauma Center, police departments of Miami and Miami Beach, and Miami-Dade MPO, FDOT Safety Office and District office, county and city planning and engineering departments, Miami-Dade county public schools, and the Florida Pedestrian Safety Center at Florida Atlantic University, among others. Also, the process of geocoding all pedestrian and bicycle crashes is expected to continue, and will allow for future identification of high-pedestrian crash locations and zones for countermeasure consideration. Another direct result of this NHTSA demonstration is that pedestrian safety is now a higher priority within Miami-Dade County, which everyone hopes will pay dividends in reductions in pedestrian injuries and deaths for many years to come. 


\section{REFERENCES}

Blomberg, R. D., \& Cleven, A. M. Zone Guide for Pedestrian Safety. July 1998. NHTSADOT/HS-808/742. Washington, DC: National Highway Traffic Safety Administration and Federal Highway Administration.

Blomberg, R. D., \& Cleven, A. M. Development, Implementation, and Evaluation of a Countermeasure Program for Alcohol-Involved Pedestrian Crashes. Final Report, DOT HS 809 067, July 2000. Washington, DC: National Highway Traffic Safety Administration.

Blomberg, R. D., \& Cleven, A. M. Development, Implementation and Evaluation of Pedestrian Safety Zone for Elderly Pedestrians. Final Report, DOT HS 808 692, February 1998.

Washington, DC: National Highway Traffic Safety Administration.

Blomberg, R. D., Cleven, A. M., \& Edwards, J. M. Development of Safety Information Materials and Media Plans for Elderly Pedestrians. Final Report, Dunlap and Associates, Inc., Norwalk, CT, June 1993. DOT HS 808 132. Washington, DC: National Highway Traffic Safety Administration.

Blomberg, R. D., Hale, A., \& Preusser, D. F. Conspicuity for Pedestrians and Bicyclists: Definition of the Problem, Development and Test of Countermeasures. Final Report. DOT HS 806 563, April 1984. Washington, DC: National Highway Traffic Safety Administration.

Blomberg, R. D., Preusser, D. F., Hale, A., \& Leaf, W. A. Experimental Field Test of Proposed Pedestrian Safety Messages: 3 Volumes, Vol. II: Child Messages. Final Report November, 1983. DOT HS 806 522. Washington, DC: National Highway Traffic Safety Administration

Blomberg, R. D., Preusser, D. F., Hale, A., \& Ulmer, R.G. A Comparison of Alcohol Involvement in Pedestrians and Pedestrian Casualties. Final Report, DOT HS 805 249, October 1979.

Washington, DC: National Highway Traffic Safety Administration.

Box, G. E. P., \& Jenkins, G. M. (1970). Time Series Analysis: Forecasting and Control. San Francisco, CA: Holden Day.

Box, G. E. P., \& Tiao, G. C. (1975). Intervention analysis with applications to econometric and environmental problems. Journal of the American Statistical Association, 70, 70-79.

Census 2000 Summary File 3. Washington, DC: Bureau of the Census. Available online at http://factfinder.census.gov/servlet/DCGeoSelectServlet?ds_name=DEC_2000_SF3_U, 2000.

Cleven, A. M., \& Blomberg, R. D. A Compendium of NHTSA Pedestrian and Bicyclist Research Projects: 1969 - 2005. January 2007. Washington, DC: National Highway Traffic Safety Administration.

Harkey, D., \& Zegeer, C. V. PEDSAFE: Pedestrian Safety Guide and Countermeasure Selection System. September 2004. Washington, DC: Federal Highway Administration. 
Harkey, D. L., Tsai, S., Thomas, L., \& Hunter, W. W. Pedestrian and Bicycle Crash Analysis Tool. March 2006. Report No. FHWA-HRT-06-089. Washington, DC: Federal Highway Administration.

Hale, A., \& Zeidler, P. Review of the Literature and Programs for Pedestrian and Bicyclist Conspicuity. Final Report, DOT HS-806 564, April 1984. Washington, DC: National Highway Traffic Safety Administration.

Hotz, G. A., Cohn, S. M., Nelson J., Mishkin D., Castelblanco A., Li, P., \& Duncan R. Pediatric Pedestrian Injury Task Force. Traffic Inj Prev 2004; 5(2): 132-136.

Hunter, W. W., Stutts, J. C., Pein, W. E., \& Cox, C. L. Pedestrian and Bicycle Crash Types of the Early 1990’s. June 1996. FHWA-RD-95-163. Washington, DC: Federal Highway Administration.

Knoblauch, R. L. Causative Factors and Countermeasures for Rural and Suburban Pedestrian Accidents: Accident Data Collection and Analysis. March 1977. DOT-HS-355-3-718 Washington, DC: National Highway Traffic Safety Administration and Federal Highway Administration.

Leaf, W. A., \& Preusser, D. F. Identification of Alcohol-Pedestrian Crash Problems Among Selected Racial/Ethnic Groups. Final Report. September 1997. DOT HS 808641 Washington, DC: National Highway Traffic Safety Administration.

Levine, N. CrimeStat III: A Spatial Statistics Program for the Analysis of Crime Incident Locations (version 3.0). 2004, Ned Levine \& Associates: Houston, TX. Washington, DC: National Institute of Justice.

Liu, L-M. (2006). Time series analysis and forecasting ( $2^{\text {nd }}$ ed.). River Forest, IL: Scientific Computing Associates.

McCleary, R., \& Hay, R.A. (1980). Applied time series analysis for the social sciences. Newbury Park, CA: Sage Publications.

National Center for Statistics and Analysis. Traffic Safety Facts 2005. DOT HS 810631. Washington, DC: National Highway Traffic Safety Administration.

Preusser, D. F., \& Lund, A. K. (1988). And Keep on Looking: A Film to Reduce Pedestrian Crashes Among 9- to 12-Year-Olds, Journal of Safety Research, Vol 19:177-185. Washington, DC: National Highway Traffic Safety Administration.

Snyder, M. B., \& Knoblauch, R. L. Pedestrian safety: the identification of precipitating factors and possible countermeasures, Volume 1. Final Report, DOT HS-800 403, January 1971. Washington, DC: National Highway Traffic Safety Administration. 
Stutts, J. C., Hunter, W. W., Tracy, L., \& Wilkinson, III, W. C. (1992) Pedestrian and Bicyclist Safety: A Review of Key Program and Countermeasure Developments During the 1980's. HSRC-PR187. University of North Carolina Highway Safety Research Center: Chapel Hill, NC, 1992.

Thackray, Jr., R. M., \& Chiplock, L. W. The Denver Pedestrian Safety Project. Applied Science Associates, Inc. and Colorado Division of Highway Safety, 1981.

University of Florida, Final Problem Identification, Countermeasure Selection, and Outreach and Awareness Report. December 5, 2002. Washington, DC: Federal Highway Administration. accessed February 23, 2007, online at http://www.dot.state.fl.us/ResearchCenter/Completed_Proj/Summary_SF/FDOT_BC354_63_rpt.pdf,

Van Houten, R., \& Malenfant, J. E. L. The Effects of a Behavioral Pedestrian Enforcement Program on Yielding Behavior in the City of Miami Beach. Center for Education and Research in Safety, June 23, 2003.

Van Houten, R., \& Malenfant, J. E. L. The influence of signs prompting motorists to yield before marked crosswalks on motor vehicle-pedestrian conflicts at crosswalks with flashing amber. Accident Analysis and Prevention, 1992; 24(3): 217-225.

Krishnakumar, V. K., Pulugurtha, S. S., \& Nambisan, S. S. Identification and Ranking of High Pedestrian Crash Zones Using GIS. 2005 Annual ESRI International User Conference, San Diego, California. July 25-29, 2005.

Yaffee, R. A. (2000). Introduction to Time Series Analysis and Forecasting with Applications of SAS and SPSS. New York: Academic Press, Inc.

Zylman, R., Blomberg, R. D., \& Preusser, D. F. A Review of the Literature on the Involvement of Alcohol in Pedestrian Collisions Resulting in Death and Injury. Interim Report, DOT HS 801 413, February 1975. Washington, DC: National Highway Traffic Safety Administration. 


\section{APPENDIX A: CRASH TYPING CODES}

For example graphics of each crash group, see Table 3 or go to http://www.walkinginfo.org/facts/pbcat/ped_images.cfm.

\begin{tabular}{|c|c|c|}
\hline Crash Group Name and Description* & $\begin{array}{l}\text { PBCAT } \\
\text { (Version 1), } \\
\text { Crash Type } \\
\text { Number }\end{array}$ & $\begin{array}{c}\text { PBCAT Crash Type } \\
\text { Name }\end{array}$ \\
\hline \multirow{2}{*}{$\begin{array}{l}\text { Dash/Dart-Out-The pedestrian walked or ran into the roadway } \\
\text { at an intersection or midblock location and was struck by a } \\
\text { vehicle. The motorist's view of the pedestrian may have been } \\
\text { blocked until an instant before the impact. }\end{array}$} & 741 & Dash \\
\hline & 742 & Dart-Out \\
\hline \multirow{2}{*}{$\begin{array}{l}\text { Multiple Threat I Trapped-The pedestrian entered the } \\
\text { roadway in front of stopped or slowed traffic and was struck by } \\
\text { a multiple-threat vehicle in an adjacent lane after becoming } \\
\text { trapped in the middle of the roadway. }\end{array}$} & 710 & Multiple Threat \\
\hline & 730 & Trapped \\
\hline \multirow{5}{*}{$\begin{array}{l}\text { Unique Midblock-The pedestrian was struck while crossing } \\
\text { the road to/from a mailbox, newspaper box, or ice-cream truck, } \\
\text { or while getting into or out of a stopped vehicle. }\end{array}$} & 320 & $\begin{array}{l}\text { Entering / Exiting } \\
\text { Parked Vehicle }\end{array}$ \\
\hline & 331 & $\begin{array}{l}\text { Mailbox-Related - } \\
\text { crossing roadway }\end{array}$ \\
\hline & 332 & $\begin{array}{l}\text { Mailbox-Related - } \\
\text { standing at mailbox }\end{array}$ \\
\hline & 339 & $\begin{array}{l}\text { Mailbox-Related - } \\
\text { other/unknown }\end{array}$ \\
\hline & 360 & $\begin{array}{l}\text { Ice Cream / Vendor } \\
\text { Truck-Related }\end{array}$ \\
\hline \multirow{5}{*}{$\begin{array}{l}\text { Crossing Roadway - Vehicle Not Turning-The pedestrian } \\
\text { was struck at an unsignalized intersection or midblock location. } \\
\text { Either the motorist or the pedestrian may have failed to yield. }\end{array}$} & 761 & $\begin{array}{l}\text { Pedestrian Failed to } \\
\text { Yield - walked into } \\
\text { vehicle }\end{array}$ \\
\hline & 762 & $\begin{array}{l}\text { Pedestrian Failed to } \\
\text { Yield - misjudged gap }\end{array}$ \\
\hline & 763 & $\begin{array}{l}\text { Pedestrian Failed to } \\
\text { Yield - step-out }\end{array}$ \\
\hline & 769 & $\begin{array}{l}\text { Pedestrian Failed to } \\
\text { Yield - other }\end{array}$ \\
\hline & 770 & Motorist Failed to Yield \\
\hline \multirow{2}{*}{$\begin{array}{l}\text { Bus-Related-The pedestrian was struck by a vehicle while: (1) } \\
\text { crossing in front of a commercial bus stopped at a bus stop; ( } 2 \text { ) } \\
\text { going to or from a school bus stop; or (3) going to or from, or } \\
\text { waiting near a commercial bus stop. }\end{array}$} & 341 & $\begin{array}{l}\text { Commercial Bus- } \\
\text { Related }\end{array}$ \\
\hline & 342 & School Bus-Related \\
\hline \multirow{4}{*}{$\begin{array}{l}\text { Crossing Roadway - Vehicle Turning-The pedestrian was } \\
\text { attempting to cross at an intersection, driveway, or alley and } \\
\text { was struck by a vehicle that was turning right or left. }\end{array}$} & 721 & $\begin{array}{l}\text { Right-Turn - same } \\
\text { direction }\end{array}$ \\
\hline & 722 & $\begin{array}{l}\text { Right-Turn - opposite } \\
\text { direction }\end{array}$ \\
\hline & 723 & $\begin{array}{l}\text { Left-Turn - same } \\
\text { direction }\end{array}$ \\
\hline & 724 & $\begin{array}{l}\text { Left-Turn - opposite } \\
\text { direction }\end{array}$ \\
\hline
\end{tabular}




\begin{tabular}{|c|c|c|}
\hline Crash Group Name and Description* & $\begin{array}{l}\text { PBCAT } \\
\text { (Version 1), } \\
\text { Crash Type } \\
\text { Number }\end{array}$ & $\begin{array}{l}\text { PBCAT Crash Type } \\
\text { Name }\end{array}$ \\
\hline & 729 & $\begin{array}{l}\text { Turn/Merge - direction } \\
\text { unknown }\end{array}$ \\
\hline \multirow{2}{*}{$\begin{array}{l}\text { Crossing Driveway or Alley-Vehicle entered or exited a } \\
\text { driveway or alley and struck pedestrian. }\end{array}$} & 810 & $\begin{array}{l}\text { Off-Roadway - vehicle } \\
\text { entering driveway/alley }\end{array}$ \\
\hline & 820 & $\begin{array}{l}\text { Off-Roadway - vehicle } \\
\text { exiting driveway/alley }\end{array}$ \\
\hline \multirow{2}{*}{$\begin{array}{l}\text { Crossing Roadway - Vehicle Not Turning-The pedestrian } \\
\text { was struck at an unsignalized intersection or midblock location. } \\
\text { Either the motorist or the pedestrian may have failed to yield. }\end{array}$} & 760 & $\begin{array}{l}\text { Pedestrian Failed to } \\
\text { Yield }\end{array}$ \\
\hline & 770 & Motorist Failed to Yield \\
\hline \multirow{5}{*}{$\begin{array}{l}\text { Walking Along Roadway-The pedestrian was walking or } \\
\text { running along the roadway and was struck from the front or from } \\
\text { behind by a vehicle. }\end{array}$} & 410 & $\begin{array}{l}\text { Walking Along } \\
\text { Roadway With Traffic - } \\
\text { From Behind }\end{array}$ \\
\hline & 420 & $\begin{array}{l}\text { Walking Along } \\
\text { Roadway With Traffic - } \\
\text { From Front }\end{array}$ \\
\hline & 430 & $\begin{array}{l}\text { Walking Along } \\
\text { Roadway Against } \\
\text { Traffic - From Behind }\end{array}$ \\
\hline & 440 & $\begin{array}{l}\text { Walking Along } \\
\text { Roadway Against } \\
\text { Traffic - From Front }\end{array}$ \\
\hline & 490 & $\begin{array}{l}\text { Walking Along } \\
\text { Roadway - Direction / } \\
\text { Position Unknown }\end{array}$ \\
\hline \multirow{2}{*}{$\begin{array}{l}\text { Working or Playing in Roadway-A vehicle struck a } \\
\text { pedestrian who was: (1) standing or walking near a disabled } \\
\text { vehicle, (2) riding a play vehicle that was not a bicycle (e.g., } \\
\text { wagon, sled, tricycle, skates), (3) playing in the road, or (4) } \\
\text { working in the road. }\end{array}$} & 311 & Working in Roadway \\
\hline & 312 & Playing in Roadway \\
\hline \multirow{2}{*}{$\begin{array}{l}\text { Off Roadway-The pedestrian was standing or walking near } \\
\text { the roadway edge, on the sidewalk, in a driveway or alley, or in } \\
\text { a parking lot, when struck by a vehicle. }\end{array}$} & 830 & $\begin{array}{l}\text { Off Roadway - Parking } \\
\text { Lot }\end{array}$ \\
\hline & 890 & $\begin{array}{l}\text { Off Roadway - Other I } \\
\text { Unknown }\end{array}$ \\
\hline \multirow{5}{*}{$\begin{array}{l}\text { Backing Vehicle-The pedestrian was struck by a backing } \\
\text { vehicle on a street, in a driveway, on a sidewalk, in a parking } \\
\text { lot, or at another location. }\end{array}$} & 211 & $\begin{array}{l}\text { Backing Vehicle - } \\
\text { Driveway }\end{array}$ \\
\hline & 212 & $\begin{array}{l}\text { Backing Vehicle - } \\
\text { Driveway / Sidewalk } \\
\text { Intersection }\end{array}$ \\
\hline & 213 & $\begin{array}{l}\text { Backing Vehicle - } \\
\text { Roadway }\end{array}$ \\
\hline & 214 & $\begin{array}{l}\text { Backing Vehicle - } \\
\text { Parking Lot }\end{array}$ \\
\hline & 219 & $\begin{array}{l}\text { Backing Vehicle - Other } \\
\text { / Unknown }\end{array}$ \\
\hline
\end{tabular}




\begin{tabular}{|c|c|c|}
\hline Crash Group Name and Description* & $\begin{array}{c}\text { PBCAT } \\
\text { (Version 1), } \\
\text { Crash Type } \\
\text { Number }\end{array}$ & $\begin{array}{l}\text { PBCAT Crash Type } \\
\text { Name }\end{array}$ \\
\hline $\begin{array}{l}\text { Crossing Expressway-The pedestrian was struck while } \\
\text { crossing a limited-access expressway or expressway ramp. }\end{array}$ & 910 & $\begin{array}{l}\text { Crossing an } \\
\text { Expressway }\end{array}$ \\
\hline \multirow{11}{*}{$\begin{array}{l}\text { Unusual Circumstances-The pedestrian was struck during } \\
\text { unusual circumstances, such as an assault with a vehicle, a } \\
\text { dispute, loss of control, driverless vehicle, or other unusual } \\
\text { situation. }\end{array}$} & 110 & Assault with Vehicle \\
\hline & 120 & Dispute-Related \\
\hline & 130 & Pedestrian on Vehicle \\
\hline & 140 & Vehicle-Vehicle / Object \\
\hline & 150 & $\begin{array}{l}\text { Motor Vehicle Loss of } \\
\text { Control }\end{array}$ \\
\hline & 160 & $\begin{array}{l}\text { Pedestrian Loss of } \\
\text { Control }\end{array}$ \\
\hline & 190 & $\begin{array}{l}\text { Other Unusual } \\
\text { Circumstances }\end{array}$ \\
\hline & 220 & Driverless Vehicle \\
\hline & 230 & $\begin{array}{l}\text { Disabled Vehicle- } \\
\text { Related }\end{array}$ \\
\hline & 240 & $\begin{array}{l}\text { Emergency Vehicle- } \\
\text { Related }\end{array}$ \\
\hline & 250 & Play Vehicle-Related \\
\hline \multirow{3}{*}{$\begin{array}{l}\text { Waiting to Cross-Pedestrian was struck while waiting to } \\
\text { cross a roadway, standing at or near curb. }\end{array}$} & 510 & $\begin{array}{l}\text { Waiting to Cross - } \\
\text { Vehicle Turning }\end{array}$ \\
\hline & 520 & $\begin{array}{l}\text { Waiting to Cross - } \\
\text { Vehicle Not Turning }\end{array}$ \\
\hline & 590 & $\begin{array}{l}\text { Waiting to Cross - } \\
\text { Vehicle Action } \\
\text { Unknown }\end{array}$ \\
\hline \multirow{3}{*}{$\begin{array}{l}\text { Pedestrian in Roadway - Circumstances Unknown-The } \\
\text { pedestrian was struck while walking, standing, or lying in the } \\
\text { roadway but the pre-crash circumstances were not known. }\end{array}$} & 620 & Walking in Roadway \\
\hline & 610 & Standing in Roadway \\
\hline & 313 & Lying in Roadway \\
\hline \multirow{3}{*}{$\begin{array}{l}\text { Other I Unknown - Insufficient Details-There was } \\
\text { insufficient information to determine a crash type. }\end{array}$} & 900 & $\begin{array}{l}\text { Other - Unknown } \\
\text { Location }\end{array}$ \\
\hline & 680 & $\begin{array}{l}\text { Non-Intersection - Other } \\
\text { / Unknown }\end{array}$ \\
\hline & 690 & $\begin{array}{l}\text { Intersection - Other / } \\
\text { Unknown }\end{array}$ \\
\hline
\end{tabular}


APPENDIX B: PEDESTRIAN CRASH COUNTS AND POPULATION FOR MIAMI-DADE AND CONTROLS

\begin{tabular}{|c|c|c|c|c|c|c|c|c|}
\hline \multirow[b]{2}{*}{ Date } & \multicolumn{4}{|c|}{ All Pedestrian Crashes } & \multicolumn{4}{|c|}{ Population } \\
\hline & Miami-Dade & Broward & $\begin{array}{c}\text { Six } \\
\text { Metro. } \\
\text { Counties }\end{array}$ & $\begin{array}{c}\text { Statewide- } \\
\text { No Dade }\end{array}$ & Miami-Dade & Broward & $\begin{array}{c}\text { Six } \\
\text { Metro. } \\
\text { Counties }\end{array}$ & $\begin{array}{c}\text { Statewide- } \\
\text { No Dade }\end{array}$ \\
\hline Mar-95 & & & & & $2,083,721$ & $1,438,108$ & $5,717,695$ & $12,321,734$ \\
\hline Apr-95 & & & & & $2,086,927$ & $1,441,339$ & $5,727,825$ & $12,343,181$ \\
\hline May-95 & & & & & $2,090,132$ & $1,444,570$ & $5,737,955$ & $12,364,628$ \\
\hline Jun-95 & & & & & $2,093,338$ & $1,447,800$ & $5,748,085$ & $12,386,075$ \\
\hline Jul-95 & & & & & $2,096,544$ & $1,451,031$ & $5,758,214$ & $12,407,522$ \\
\hline Aug-95 & & & & & $2,099,749$ & $1,454,262$ & $5,768,344$ & $12,428,969$ \\
\hline Sep-95 & & & & & $2,102,955$ & $1,457,493$ & $5,778,474$ & $12,450,417$ \\
\hline Oct-95 & & & & & $2,106,161$ & $1,460,723$ & $5,788,604$ & $12,471,864$ \\
\hline Nov-95 & & & & & $2,109,366$ & $1,463,954$ & $5,798,734$ & $12,493,311$ \\
\hline Dec-95 & & & & & $2,112,572$ & $1,467,185$ & $5,808,864$ & $12,514,758$ \\
\hline Jan-96 & 192 & 100 & 352 & 625 & $2,115,778$ & $1,470,416$ & $5,818,993$ & $12,536,205$ \\
\hline Feb-96 & 183 & 102 & 357 & 600 & $2,118,983$ & $1,473,646$ & $5,829,123$ & $12,557,652$ \\
\hline Mar-96 & 205 & 95 & 354 & 646 & $2,122,189$ & $1,476,877$ & $5,839,253$ & $12,579,099$ \\
\hline Apr-96 & 157 & 99 & 317 & 566 & $2,124,733$ & $1,480,113$ & $5,849,843$ & $12,602,422$ \\
\hline May-96 & 169 & 85 & 302 & 543 & $2,127,278$ & $1,483,349$ & $5,860,432$ & $12,625,745$ \\
\hline Jun-96 & 134 & 69 & 266 & 466 & $2,129,822$ & $1,486,586$ & $5,871,022$ & $12,649,067$ \\
\hline Jul-96 & 139 & 69 & 266 & 485 & $2,132,366$ & $1,489,822$ & $5,881,611$ & $12,672,390$ \\
\hline Aug-96 & 148 & 71 & 275 & 500 & $2,134,910$ & $1,493,058$ & $5,892,201$ & $12,695,713$ \\
\hline Sep-96 & 133 & 80 & 323 & 568 & $2,137,455$ & $1,496,294$ & $5,902,790$ & $12,719,036$ \\
\hline Oct-96 & 163 & 92 & 368 & 638 & $2,139,999$ & $1,499,530$ & $5,913,380$ & $12,742,358$ \\
\hline
\end{tabular}




\begin{tabular}{|c|c|c|c|c|c|c|c|c|}
\hline \multirow[b]{2}{*}{ Date } & \multicolumn{4}{|c|}{ All Pedestrian Crashes } & \multicolumn{4}{|c|}{ Population } \\
\hline & Miami-Dade & Broward & $\begin{array}{c}\text { Six } \\
\text { Metro. } \\
\text { Counties }\end{array}$ & $\begin{array}{c}\text { Statewide- } \\
\text { No Dade }\end{array}$ & Miami-Dade & Broward & $\begin{array}{c}\text { Six } \\
\text { Metro. } \\
\text { Counties }\end{array}$ & $\begin{array}{c}\text { Statewide- } \\
\text { No Dade }\end{array}$ \\
\hline Nov-96 & 171 & 82 & 373 & 643 & $2,142,543$ & $1,502,766$ & $5,923,969$ & $12,765,681$ \\
\hline Dec-96 & 168 & 94 & 347 & 641 & $2,145,087$ & $1,506,003$ & $5,934,559$ & $12,789,004$ \\
\hline Jan-97 & 194 & 102 & 350 & 615 & $2,147,632$ & $1,509,239$ & $5,945,148$ & $12,812,327$ \\
\hline Feb-97 & 153 & 88 & 320 & 572 & $2,150,176$ & $1,512,475$ & $5,955,738$ & $12,835,649$ \\
\hline Mar-97 & 213 & 113 & 383 & 721 & $2,152,720$ & $1,515,711$ & $5,966,327$ & $12,858,972$ \\
\hline Apr-97 & 168 & 89 & 324 & 592 & $2,154,989$ & $1,518,655$ & $5,976,021$ & $12,881,557$ \\
\hline May-97 & 150 & 76 & 330 & 592 & $2,157,258$ & $1,521,599$ & $5,985,715$ & $12,904,142$ \\
\hline Jun-97 & 170 & 86 & 281 & 480 & $2,159,526$ & $1,524,543$ & $5,995,408$ & $12,926,728$ \\
\hline Jul-97 & 142 & 71 & 261 & 476 & $2,161,795$ & $1,527,487$ & $6,005,102$ & $12,949,313$ \\
\hline Aug-97 & 152 & 76 & 323 & 561 & $2,164,064$ & $1,530,431$ & $6,014,796$ & $12,971,898$ \\
\hline Sep-97 & 150 & 90 & 281 & 527 & $2,166,333$ & $1,533,375$ & $6,024,490$ & $12,994,483$ \\
\hline Oct-97 & 156 & 76 & 325 & 605 & $2,168,601$ & $1,536,319$ & $6,034,183$ & $13,017,068$ \\
\hline Nov-97 & 142 & 83 & 305 & 527 & $2,170,870$ & $1,539,263$ & $6,043,877$ & $13,039,653$ \\
\hline Dec-97 & 151 & 114 & 389 & 651 & $2,173,139$ & $1,542,207$ & $6,053,571$ & $13,062,239$ \\
\hline Jan-98 & 190 & 111 & 371 & 640 & $2,175,408$ & $1,545,151$ & $6,063,265$ & $13,084,824$ \\
\hline Feb-98 & 182 & 102 & 369 & 635 & $2,177,676$ & $1,548,095$ & $6,072,958$ & $13,107,409$ \\
\hline Mar-98 & 188 & 105 & 381 & 717 & $2,179,945$ & $1,551,039$ & $6,082,652$ & $13,129,994$ \\
\hline Apr-98 & 167 & 73 & 379 & 664 & $2,183,227$ & $1,554,316$ & $6,094,504$ & $13,157,517$ \\
\hline May-98 & 176 & 85 & 313 & 563 & $2,186,509$ & $1,557,593$ & $6,106,357$ & $13,185,041$ \\
\hline Jun-98 & 149 & 92 & 317 & 542 & $2,189,791$ & $1,560,870$ & $6,118,209$ & $13,212,564$ \\
\hline Jul-98 & 152 & 82 & 310 & 519 & $2,193,073$ & $1,564,146$ & $6,130,062$ & $13,240,087$ \\
\hline Aug-98 & 119 & 80 & 272 & 475 & $2,196,355$ & $1,567,423$ & $6,141,914$ & $13,267,611$ \\
\hline
\end{tabular}




\begin{tabular}{|c|c|c|c|c|c|c|c|c|}
\hline \multirow[b]{2}{*}{ Date } & \multicolumn{4}{|c|}{ All Pedestrian Crashes } & \multicolumn{4}{|c|}{ Population } \\
\hline & Miami-Dade & Broward & $\begin{array}{c}\text { Six } \\
\text { Metro. } \\
\text { Counties }\end{array}$ & $\begin{array}{c}\text { Statewide- } \\
\text { No Dade }\end{array}$ & Miami-Dade & Broward & $\begin{array}{c}\text { Six } \\
\text { Metro. } \\
\text { Counties }\end{array}$ & $\begin{array}{c}\text { Statewide- } \\
\text { No Dade }\end{array}$ \\
\hline Sep-98 & 145 & 88 & 299 & 528 & $2,199,637$ & $1,570,700$ & $6,153,767$ & $13,295,134$ \\
\hline Oct-98 & 197 & 105 & 377 & 673 & $2,202,919$ & $1,573,977$ & $6,165,619$ & $13,322,657$ \\
\hline Nov-98 & 139 & 101 & 351 & 627 & $2,206,201$ & $1,577,254$ & $6,177,471$ & $13,350,181$ \\
\hline Dec-98 & 171 & 110 & 427 & 723 & $2,209,483$ & $1,580,531$ & $6,189,324$ & $13,377,704$ \\
\hline Jan-99 & 187 & 85 & 352 & 615 & $2,212,765$ & $1,583,807$ & $6,201,176$ & $13,405,227$ \\
\hline Feb-99 & 173 & 110 & 331 & 597 & $2,216,047$ & $1,587,084$ & $6,213,029$ & $13,432,751$ \\
\hline Mar-99 & 170 & 85 & 375 & 675 & $2,219,329$ & $1,590,361$ & $6,224,881$ & $13,460,274$ \\
\hline Apr-99 & 158 & 110 & 352 & 607 & $2,222,960$ & $1,593,785$ & $6,238,373$ & $13,489,573$ \\
\hline Мay-99 & 152 & 84 & 305 & 545 & $2,226,591$ & $1,597,208$ & $6,251,865$ & $13,518,872$ \\
\hline Jun-99 & 141 & 85 & 286 & 522 & $2,230,222$ & $1,600,632$ & $6,265,357$ & $13,548,171$ \\
\hline Jul-99 & 144 & 58 & 249 & 468 & $2,233,853$ & $1,604,056$ & $6,278,849$ & $13,577,470$ \\
\hline Aug-99 & 151 & 71 & 293 & 489 & $2,237,484$ & $1,607,479$ & $6,292,341$ & $13,606,769$ \\
\hline Sep-99 & 159 & 79 & 280 & 494 & $2,241,116$ & $1,610,903$ & $6,305,833$ & $13,636,068$ \\
\hline Oct-99 & 173 & 95 & 358 & 641 & $2,244,747$ & $1,614,327$ & $6,319,324$ & $13,665,366$ \\
\hline Nov-99 & 142 & 94 & 379 & 661 & $2,248,378$ & $1,617,750$ & $6,332,816$ & $13,694,665$ \\
\hline Dec-99 & 165 & 85 & 322 & 586 & $2,252,009$ & $1,621,174$ & $6,346,308$ & $13,723,964$ \\
\hline Jan-00 & 203 & 102 & 358 & 601 & $2,255,640$ & $1,624,598$ & $6,359,800$ & $13,753,263$ \\
\hline Feb-00 & 184 & 118 & 399 & 681 & $2,259,271$ & $1,628,021$ & $6,373,292$ & $13,782,562$ \\
\hline Mar-00 & 190 & 104 & 396 & 727 & $2,262,902$ & $1,631,445$ & $6,386,784$ & $13,811,861$ \\
\hline Apr-00 & 153 & 82 & 347 & 613 & $2,265,353$ & $1,633,402$ & $6,397,467$ & $13,837,532$ \\
\hline May-00 & 161 & 91 & 316 & 565 & $2,267,804$ & $1,635,358$ & $6,408,150$ & $13,863,202$ \\
\hline Jun-00 & 168 & 82 & 266 & 475 & $2,270,256$ & $1,637,315$ & $6,418,833$ & $13,888,873$ \\
\hline
\end{tabular}




\begin{tabular}{|c|c|c|c|c|c|c|c|c|}
\hline \multirow[b]{2}{*}{ Date } & \multicolumn{4}{|c|}{ All Pedestrian Crashes } & \multicolumn{4}{|c|}{ Population } \\
\hline & Miami-Dade & Broward & $\begin{array}{c}\text { Six } \\
\text { Metro. } \\
\text { Counties }\end{array}$ & $\begin{array}{l}\text { Statewide- } \\
\text { No Dade }\end{array}$ & Miami-Dade & Broward & $\begin{array}{c}\text { Six } \\
\text { Metro. } \\
\text { Counties }\end{array}$ & $\begin{array}{l}\text { Statewide- } \\
\text { No Dade }\end{array}$ \\
\hline Jul-00 & 133 & 76 & 279 & 479 & $2,272,707$ & $1,639,271$ & $6,429,516$ & $13,914,544$ \\
\hline Aug-00 & 156 & 67 & 270 & 501 & $2,275,158$ & $1,641,228$ & $6,440,199$ & $13,940,214$ \\
\hline Sep-00 & 155 & 86 & 317 & 582 & $2,277,609$ & $1,643,184$ & $6,450,883$ & $13,965,885$ \\
\hline Oct-00 & 164 & 103 & 358 & 611 & $2,280,060$ & $1,645,141$ & $6,461,566$ & $13,991,556$ \\
\hline Nov-00 & 157 & 99 & 367 & 614 & $2,282,511$ & $1,647,097$ & $6,472,249$ & $14,017,226$ \\
\hline Dec-00 & 172 & 95 & 353 & 601 & $2,284,963$ & $1,649,054$ & $6,482,932$ & $14,042,897$ \\
\hline Jan-01 & 167 & 107 & 354 & 591 & $2,287,414$ & $1,651,010$ & $6,493,615$ & $14,068,568$ \\
\hline Feb-01 & 171 & 105 & 364 & 610 & $2,289,865$ & $1,652,967$ & $6,504,298$ & $14,094,238$ \\
\hline Mar-01 & 194 & 109 & 383 & 699 & $2,292,316$ & $1,654,923$ & $6,514,981$ & $14,119,909$ \\
\hline Apr-01 & 156 & 88 & 336 & 608 & $2,294,662$ & $1,656,510$ & $6,525,265$ & $14,147,548$ \\
\hline May-01 & 167 & 90 & 320 & 572 & $2,297,008$ & $1,658,098$ & $6,535,549$ & $14,175,188$ \\
\hline Jun-01 & 184 & 72 & 267 & 478 & $2,299,353$ & $1,659,685$ & $6,545,833$ & $14,202,827$ \\
\hline Jul-01 & 166 & 86 & 288 & 522 & $2,301,699$ & $1,661,273$ & $6,556,116$ & $14,230,466$ \\
\hline Aug-01 & 164 & 74 & 297 & 555 & $2,304,045$ & $1,662,860$ & $6,566,400$ & $14,258,106$ \\
\hline Sep-01 & 146 & 69 & 309 & 528 & $2,306,391$ & $1,664,448$ & $6,576,684$ & $14,285,745$ \\
\hline Oct-01 & 194 & 108 & 407 & 673 & $2,308,736$ & $1,666,035$ & $6,586,968$ & $14,313,384$ \\
\hline Nov-01 & 164 & 93 & 343 & 605 & $2,311,082$ & $1,667,622$ & $6,597,252$ & $14,341,024$ \\
\hline Dec-01 & 214 & 102 & 372 & 651 & $2,313,428$ & $1,669,210$ & $6,607,536$ & $14,368,663$ \\
\hline Jan-02 & 170 & 99 & 344 & 583 & $2,315,774$ & $1,670,797$ & $6,617,819$ & $14,396,302$ \\
\hline Feb-02 & 157 & 81 & 298 & 535 & $2,318,119$ & $1,672,385$ & $6,628,103$ & $14,423,942$ \\
\hline Mar-02 & 195 & 126 & 374 & 693 & $2,320,465$ & $1,673,972$ & $6,638,387$ & $14,451,581$ \\
\hline Apr-02 & 155 & 93 & 344 & 626 & $2,323,293$ & $1,676,671$ & $6,649,482$ & $14,481,432$ \\
\hline
\end{tabular}




\begin{tabular}{|c|c|c|c|c|c|c|c|c|}
\hline \multirow[b]{2}{*}{ Date } & \multicolumn{4}{|c|}{ All Pedestrian Crashes } & \multicolumn{4}{|c|}{ Population } \\
\hline & Miami-Dade & Broward & $\begin{array}{c}\text { Six } \\
\text { Metro. } \\
\text { Counties }\end{array}$ & $\begin{array}{c}\text { Statewide- } \\
\text { No Dade }\end{array}$ & Miami-Dade & Broward & $\begin{array}{c}\text { Six } \\
\text { Metro. } \\
\text { Counties }\end{array}$ & $\begin{array}{c}\text { Statewide- } \\
\text { No Dade }\end{array}$ \\
\hline May-02 & 168 & 85 & 287 & 550 & $2,326,122$ & $1,679,371$ & $6,660,576$ & $14,511,283$ \\
\hline Jun-02 & 130 & 74 & 242 & 434 & $2,328,950$ & $1,682,070$ & $6,671,671$ & $14,541,135$ \\
\hline Jul-02 & 148 & 66 & 241 & 461 & $2,331,778$ & $1,684,769$ & $6,682,766$ & $14,570,986$ \\
\hline Aug-02 & 150 & 76 & 319 & 545 & $2,334,606$ & $1,687,468$ & $6,693,860$ & $14,600,837$ \\
\hline Sep-02 & 145 & 85 & 299 & 551 & $2,337,435$ & $1,690,168$ & $6,704,955$ & $14,630,688$ \\
\hline Oct-02 & 164 & 95 & 322 & 596 & $2,340,263$ & $1,692,867$ & $6,716,050$ & $14,660,539$ \\
\hline Nov-02 & 183 & 80 & 338 & 593 & $2,343,091$ & $1,695,566$ & $6,727,144$ & $14,690,390$ \\
\hline Dec-02 & 181 & 95 & 342 & 611 & $2,345,919$ & $1,698,265$ & $6,738,239$ & $14,720,242$ \\
\hline Jan-03 & 158 & 110 & 326 & 589 & $2,348,748$ & $1,700,965$ & $6,749,334$ & $14,750,093$ \\
\hline Feb-03 & 182 & 108 & 327 & 603 & $2,351,576$ & $1,703,664$ & $6,760,428$ & $14,779,944$ \\
\hline Mar-03 & 156 & 110 & 346 & 642 & $2,354,404$ & $1,706,363$ & $6,771,523$ & $14,809,795$ \\
\hline Apr-03 & 136 & 102 & 318 & 554 & $2,357,215$ & $1,708,381$ & $6,782,638$ & $14,844,415$ \\
\hline May-03 & 142 & 80 & 273 & 505 & $2,360,026$ & $1,710,399$ & $6,793,753$ & $14,879,034$ \\
\hline Jun-03 & 136 & 87 & 287 & 484 & $2,362,838$ & $1,712,417$ & $6,804,869$ & $14,913,654$ \\
\hline Jul-03 & 135 & 67 & 238 & 446 & $2,365,649$ & $1,714,435$ & $6,815,984$ & $14,948,273$ \\
\hline Aug-03 & 136 & 79 & 286 & 543 & $2,368,460$ & $1,716,453$ & $6,827,099$ & $14,982,893$ \\
\hline Sep-03 & 135 & 94 & 316 & 548 & $2,371,271$ & $1,718,472$ & $6,838,214$ & $15,017,513$ \\
\hline Oct-03 & 153 & 90 & 344 & 629 & $2,374,082$ & $1,720,490$ & $6,849,329$ & $15,052,132$ \\
\hline Nov-03 & 150 & 92 & 311 & 568 & $2,376,893$ & $1,722,508$ & $6,860,444$ & $15,086,752$ \\
\hline Dec-03 & 146 & 108 & 350 & 617 & $2,379,705$ & $1,724,526$ & $6,871,560$ & $15,121,371$ \\
\hline Jan-04 & 167 & 97 & 359 & 609 & $2,382,516$ & $1,726,544$ & $6,882,675$ & $15,155,991$ \\
\hline Feb-04 & 138 & 104 & 319 & 565 & $2,385,327$ & $1,728,562$ & $6,893,790$ & $15,190,610$ \\
\hline
\end{tabular}




\begin{tabular}{|c|c|c|c|c|c|c|c|c|}
\hline \multirow[b]{2}{*}{ Date } & \multicolumn{4}{|c|}{ All Pedestrian Crashes } & \multicolumn{4}{|c|}{ Population } \\
\hline & Miami-Dade & Broward & $\begin{array}{c}\text { Six } \\
\text { Metro. } \\
\text { Counties }\end{array}$ & $\begin{array}{l}\text { Statewide- } \\
\text { No Dade }\end{array}$ & Miami-Dade & Broward & $\begin{array}{c}\text { Six } \\
\text { Metro. } \\
\text { Counties }\end{array}$ & $\begin{array}{c}\text { Statewide- } \\
\text { No Dade }\end{array}$ \\
\hline Mar-04 & 167 & 92 & 328 & 606 & $2,388,138$ & $1,730,580$ & $6,904,905$ & $15,225,230$ \\
\hline Apr-04 & 169 & 76 & 312 & 582 & $2,390,876$ & $1,733,383$ & $6,915,835$ & $15,251,415$ \\
\hline May-04 & 148 & 74 & 308 & 537 & $2,393,613$ & $1,736,186$ & $6,926,765$ & $15,277,600$ \\
\hline Jun-04 & 131 & 67 & 216 & 438 & $2,396,351$ & $1,738,989$ & $6,937,694$ & $15,303,784$ \\
\hline Jul-04 & 122 & 74 & 270 & 497 & $2,399,089$ & $1,741,792$ & $6,948,624$ & $15,329,969$ \\
\hline Aug-04 & 119 & 64 & 268 & 493 & $2,401,826$ & $1,744,595$ & $6,959,554$ & $15,356,154$ \\
\hline Sep-04 & 102 & 62 & 251 & 521 & $2,404,564$ & $1,747,399$ & $6,970,484$ & $15,382,339$ \\
\hline Oct-04 & 152 & 105 & 328 & 654 & $2,407,302$ & $1,750,202$ & $6,981,413$ & $15,408,523$ \\
\hline Nov-04 & 145 & 76 & 348 & 672 & $2,410,039$ & $1,753,005$ & $6,992,343$ & $15,434,708$ \\
\hline Dec-04 & 161 & 121 & 393 & 711 & $2,412,777$ & $1,755,808$ & $7,003,273$ & $15,460,893$ \\
\hline Jan-05 & & & & & $2,415,515$ & $1,758,611$ & $7,014,203$ & $15,487,078$ \\
\hline Feb-05 & & & & & $2,418,252$ & $1,761,414$ & $7,025,132$ & $15,513,262$ \\
\hline Mar-05 & & & & & $2,420,990$ & $1,764,217$ & $7,036,062$ & $15,539,447$ \\
\hline Total & $\begin{array}{l}17,308 \\
\end{array}$ & 9,679 & 35,058 & 62,579 & & & & \\
\hline
\end{tabular}


APPENDIX C: PEDESTRIAN CRASH COUNTS BY AGE GROUP, ZONE, AND MONTH

\begin{tabular}{|c|c|c|c|c|c|c|c|c|c|c|c|c|c|c|c|c|}
\hline \multirow[b]{3}{*}{ Date } & \multicolumn{4}{|c|}{ Liberty City } & \multicolumn{4}{|c|}{ Little Haiti } & \multicolumn{4}{|c|}{ Little Havana } & \multicolumn{4}{|c|}{ South Beach } \\
\hline & \multicolumn{3}{|c|}{ Age Group } & \multirow[b]{2}{*}{ Total } & \multicolumn{3}{|c|}{ Age Group } & \multirow[b]{2}{*}{ Total } & \multicolumn{3}{|c|}{ Age Group } & \multirow[b]{2}{*}{ Total } & \multicolumn{3}{|c|}{ Age Group } & \multirow[b]{2}{*}{ Total } \\
\hline & $<14$ & $\begin{array}{c}14 \text { to } \\
64\end{array}$ & $65+$ & & $<14$ & $\begin{array}{c}14 \text { to } \\
64\end{array}$ & $65+$ & & $<14$ & $\begin{array}{c}14 \text { to } \\
64\end{array}$ & $65+$ & & $<14$ & $\begin{array}{c}14 \text { to } \\
64\end{array}$ & $65+$ & \\
\hline Jan-96 & 3 & 4 & 1 & 8 & 2 & 3 & 1 & 6 & 0 & 4 & 0 & 4 & 1 & 8 & 2 & 11 \\
\hline Feb-96 & 3 & 3 & 1 & 7 & 4 & 5 & 0 & 9 & 0 & 3 & 0 & 3 & 0 & 8 & 0 & 8 \\
\hline Mar-96 & 5 & 8 & 2 & 15 & 1 & 0 & 0 & 1 & 2 & 4 & 1 & 7 & 1 & 12 & 6 & 19 \\
\hline Apr-96 & 6 & 6 & 1 & 13 & 2 & 5 & 0 & 7 & 0 & 3 & 3 & 6 & 1 & 5 & 1 & 7 \\
\hline May-96 & 3 & 7 & 1 & 11 & 2 & 2 & 1 & 5 & 3 & 6 & 1 & 10 & 1 & 6 & 1 & 8 \\
\hline Jun-96 & 3 & 8 & 0 & 11 & 1 & 1 & 1 & 3 & 1 & 3 & 2 & 6 & 0 & 2 & 2 & 4 \\
\hline Jul-96 & 2 & 7 & 2 & 11 & 2 & 1 & 0 & 3 & 0 & 9 & 3 & 12 & 1 & 5 & 1 & 7 \\
\hline Aug-96 & 1 & 6 & 3 & 10 & 2 & 2 & 1 & 5 & 0 & 5 & 0 & 5 & 0 & 9 & 1 & 10 \\
\hline Sep-96 & 2 & 5 & 1 & 8 & 3 & 1 & 1 & 5 & 1 & 4 & 1 & 6 & 0 & 5 & 2 & 7 \\
\hline Oct-96 & 3 & 4 & 0 & 7 & 2 & 2 & 0 & 4 & 0 & 5 & 4 & 9 & 1 & 5 & 0 & 6 \\
\hline Nov-96 & 3 & 1 & 0 & 4 & 3 & 4 & 0 & 7 & 1 & 6 & 4 & 11 & 0 & 5 & 3 & 8 \\
\hline Dec-96 & 4 & 8 & 1 & 13 & 1 & 3 & 0 & 4 & 0 & 4 & 1 & 5 & 0 & 6 & 1 & 7 \\
\hline Jan-97 & 3 & 6 & 0 & 9 & 1 & 2 & 0 & 3 & 2 & 3 & 1 & 6 & 0 & 7 & 3 & 10 \\
\hline Feb-97 & 5 & 5 & 1 & 11 & 0 & 3 & 0 & 3 & 0 & 4 & 0 & 4 & 0 & 5 & 2 & 7 \\
\hline Mar-97 & 6 & 8 & 2 & 16 & 2 & 1 & 2 & 5 & 0 & 6 & 2 & 8 & 1 & 7 & 1 & 9 \\
\hline Apr-97 & 3 & 11 & 0 & 14 & 1 & 3 & 0 & 4 & 2 & 2 & 2 & 6 & 1 & 4 & 1 & 6 \\
\hline May-97 & 2 & 4 & 1 & 7 & 1 & 4 & 0 & 5 & 0 & 3 & 2 & 5 & 0 & 6 & 3 & 9 \\
\hline Jun-97 & 6 & 6 & 0 & 12 & 3 & 3 & 0 & 6 & 1 & 3 & 1 & 5 & 0 & 7 & 1 & 8 \\
\hline Jul-97 & 5 & 3 & 1 & 9 & 0 & 2 & 0 & 2 & 0 & 3 & 1 & 4 & 2 & 9 & 0 & 11 \\
\hline Aug-97 & 1 & 8 & 0 & 9 & 1 & 6 & 0 & 7 & 0 & 4 & 4 & 8 & 0 & 7 & 3 & 10 \\
\hline Sep-97 & 2 & 7 & 0 & 9 & 0 & 6 & 1 & 7 & 0 & 2 & 2 & 4 & 0 & 7 & 1 & 8 \\
\hline Oct-97 & 4 & 9 & 0 & 13 & 3 & 1 & 0 & 4 & 0 & 2 & 2 & 4 & 0 & 4 & 1 & 5 \\
\hline Nov-97 & 3 & 7 & 1 & 11 & 1 & 1 & 0 & 2 & 1 & 3 & 0 & 4 & 0 & 8 & 1 & 9 \\
\hline Dec-97 & 3 & 7 & 0 & 10 & 0 & 0 & 0 & 0 & 0 & 1 & 0 & 1 & 1 & 4 & 2 & 7 \\
\hline Jan-98 & 3 & 6 & 0 & 9 & 2 & 3 & 0 & 5 & 1 & 3 & 2 & 6 & 0 & 7 & 1 & 8 \\
\hline Feb-98 & 6 & 5 & 2 & 13 & 3 & 5 & 0 & 8 & 0 & 5 & 4 & 9 & 0 & 7 & 4 & 11 \\
\hline Mar-98 & 10 & 8 & 1 & 19 & 1 & 4 & 1 & 6 & 1 & 8 & 1 & 10 & 0 & 9 & 0 & 9 \\
\hline Apr-98 & 4 & 2 & 1 & 7 & 2 & 2 & 1 & 5 & 2 & 5 & 1 & 8 & 0 & 3 & 1 & 4 \\
\hline May-98 & 2 & 3 & 0 & 5 & 0 & 5 & 0 & 5 & 0 & 9 & 2 & 11 & 1 & 5 & 0 & 6 \\
\hline Jun-98 & 4 & 5 & 0 & 9 & 2 & 2 & 1 & 5 & 1 & 6 & 5 & 12 & 0 & 5 & 3 & 8 \\
\hline Jul-98 & 2 & 4 & 1 & 7 & 0 & 2 & 0 & 2 & 1 & 5 & 1 & 7 & 0 & 6 & 0 & 6 \\
\hline Aug-98 & 1 & 3 & 0 & 4 & 1 & 1 & 1 & 3 & 0 & 0 & 0 & 0 & 0 & 5 & 0 & 5 \\
\hline
\end{tabular}

C-1 


\begin{tabular}{|c|c|c|c|c|c|c|c|c|c|c|c|c|c|c|c|c|}
\hline \multirow[b]{3}{*}{ Date } & \multicolumn{4}{|c|}{ Liberty City } & \multicolumn{4}{|c|}{ Little Haiti } & \multicolumn{4}{|c|}{ Little Havana } & \multicolumn{4}{|c|}{ South Beach } \\
\hline & \multicolumn{3}{|c|}{ Age Group } & \multirow[b]{2}{*}{ Total } & \multicolumn{3}{|c|}{ Age Group } & \multirow[b]{2}{*}{ Total } & \multicolumn{3}{|c|}{ Age Group } & \multirow[b]{2}{*}{ Total } & \multicolumn{3}{|c|}{ Age Group } & \multirow[b]{2}{*}{ Total } \\
\hline & $<14$ & $\begin{array}{c}14 \text { to } \\
64\end{array}$ & $65+$ & & $<14$ & $\begin{array}{c}14 \text { to } \\
64\end{array}$ & $65+$ & & $<14$ & $\begin{array}{c}14 \text { to } \\
64\end{array}$ & $65+$ & & $<14$ & $\begin{array}{c}14 \text { to } \\
64\end{array}$ & $65+$ & \\
\hline Sep-98 & 3 & 4 & 0 & 7 & 1 & 2 & 0 & 3 & 0 & 1 & 1 & 2 & 0 & 3 & 1 & 4 \\
\hline Oct-98 & 5 & 13 & 1 & 19 & 0 & 3 & 2 & 5 & 1 & 2 & 2 & 5 & 0 & 7 & 1 & 8 \\
\hline Nov-98 & 2 & 3 & 0 & 5 & 0 & 1 & 0 & 1 & 1 & 0 & 0 & 1 & 0 & 4 & 1 & 5 \\
\hline Dec-98 & 1 & 8 & 2 & 11 & 0 & 2 & 1 & 3 & 1 & 0 & 2 & 3 & 0 & 6 & 2 & 8 \\
\hline Jan-99 & 1 & 7 & 0 & 8 & 0 & 5 & 0 & 5 & 0 & 3 & 0 & 3 & 0 & 6 & 2 & 8 \\
\hline Feb-99 & 3 & 4 & 0 & 7 & 0 & 4 & 1 & 5 & 2 & 2 & 0 & 4 & 0 & 7 & 3 & 10 \\
\hline Mar-99 & 3 & 5 & 0 & 8 & 1 & 2 & 0 & 3 & 1 & 2 & 0 & 3 & 0 & 7 & 0 & 7 \\
\hline Apr-99 & 1 & 4 & 0 & 5 & 0 & 1 & 0 & 1 & 0 & 2 & 0 & 2 & 0 & 4 & 2 & 6 \\
\hline May-99 & 0 & 6 & 0 & 6 & 1 & 0 & 1 & 2 & 0 & 0 & 4 & 4 & 0 & 7 & 2 & 9 \\
\hline Jun-99 & 0 & 9 & 0 & 9 & 0 & 1 & 0 & 1 & 0 & 4 & 4 & 8 & 0 & 5 & 0 & 5 \\
\hline Jul-99 & 0 & 2 & 0 & 2 & 1 & 4 & 0 & 5 & 0 & 2 & 3 & 5 & 0 & 3 & 2 & 5 \\
\hline Aug-99 & 1 & 7 & 1 & 9 & 2 & 1 & 1 & 4 & 1 & 1 & 1 & 3 & 0 & 4 & 3 & 7 \\
\hline Sep-99 & 3 & 8 & 1 & 12 & 0 & 7 & 1 & 8 & 1 & 4 & 2 & 7 & 0 & 14 & 1 & 15 \\
\hline Oct-99 & 4 & 10 & 2 & 16 & 0 & 6 & 0 & 6 & 0 & 6 & 1 & 7 & 1 & 4 & 2 & 7 \\
\hline Nov-99 & 4 & 3 & 1 & 8 & 3 & 1 & 0 & 4 & 1 & 1 & 1 & 3 & 0 & 8 & 3 & 11 \\
\hline Dec-99 & 1 & 3 & 0 & 4 & 1 & 2 & 0 & 3 & 0 & 0 & 0 & 0 & 0 & 6 & 1 & 7 \\
\hline Jan-00 & 3 & 5 & 2 & 10 & 2 & 2 & 0 & 4 & 0 & 5 & 1 & 6 & 0 & 9 & 2 & 11 \\
\hline Feb-00 & 2 & 8 & 0 & 10 & 0 & 5 & 1 & 6 & 1 & 2 & 5 & 8 & 0 & 11 & 4 & 15 \\
\hline Mar-00 & 2 & 7 & 0 & 9 & 0 & 7 & 1 & 8 & 2 & 8 & 3 & 13 & 0 & 11 & 1 & 12 \\
\hline Apr-00 & 5 & 8 & 0 & 13 & 5 & 2 & 0 & 7 & 2 & 4 & 1 & 7 & 0 & 7 & 0 & 7 \\
\hline May-00 & 2 & 7 & 0 & 9 & 1 & 3 & 0 & 4 & 0 & 5 & 0 & 5 & 0 & 10 & 1 & 11 \\
\hline Jun-00 & 2 & 4 & 0 & 6 & 3 & 2 & 0 & 5 & 1 & 3 & 0 & 4 & 0 & 5 & 0 & 5 \\
\hline Jul-00 & 0 & 3 & 0 & 3 & 2 & 3 & 0 & 5 & 0 & 1 & 1 & 2 & 0 & 4 & 0 & 4 \\
\hline Aug-00 & 3 & 5 & 0 & 8 & 2 & 2 & 0 & 4 & 2 & 5 & 2 & 9 & 0 & 4 & 2 & 6 \\
\hline Sep-00 & 1 & 5 & 1 & 7 & 2 & 2 & 0 & 4 & 1 & 4 & 0 & 5 & 0 & 5 & 1 & 6 \\
\hline Oct-00 & 5 & 7 & 1 & 13 & 2 & 1 & 1 & 4 & 0 & 4 & 0 & 4 & 1 & 6 & 2 & 9 \\
\hline Nov-00 & 2 & 8 & 1 & 11 & 1 & 1 & 1 & 3 & 1 & 5 & 2 & 8 & 0 & 5 & 1 & 6 \\
\hline Dec-00 & 3 & 5 & 1 & 9 & 1 & 2 & 1 & 4 & 0 & 2 & 3 & 5 & 1 & 5 & 3 & 9 \\
\hline Jan-01 & 2 & 5 & 2 & 9 & 0 & 4 & 2 & 6 & 1 & 2 & 3 & 6 & 2 & 7 & 1 & 10 \\
\hline Feb-01 & 2 & 6 & 1 & 9 & 2 & 1 & 1 & 4 & 0 & 3 & 9 & 12 & 0 & 2 & 0 & 2 \\
\hline Mar-01 & 6 & 5 & 0 & 11 & 1 & 3 & 0 & 4 & 0 & 8 & 1 & 9 & 1 & 4 & 1 & 6 \\
\hline Apr-01 & 1 & 7 & 0 & 8 & 0 & 2 & 1 & 3 & 1 & 3 & 1 & 5 & 0 & 13 & 0 & 13 \\
\hline May-01 & 2 & 6 & 0 & 8 & 0 & 2 & 0 & 2 & 0 & 3 & 1 & 4 & 0 & 9 & 0 & 9 \\
\hline Jun-01 & 2 & 6 & 3 & 11 & 2 & 3 & 0 & 5 & 1 & 5 & 7 & 13 & 1 & 6 & 0 & 7 \\
\hline Jul-01 & 4 & 2 & 1 & 7 & 2 & 3 & 0 & 5 & 2 & 4 & 1 & 7 & 1 & 5 & 0 & 6 \\
\hline
\end{tabular}




\begin{tabular}{|c|c|c|c|c|c|c|c|c|c|c|c|c|c|c|c|c|}
\hline \multirow[b]{3}{*}{ Date } & \multicolumn{4}{|c|}{ Liberty City } & \multicolumn{4}{|c|}{ Little Haiti } & \multicolumn{4}{|c|}{ Little Havana } & \multicolumn{4}{|c|}{ South Beach } \\
\hline & \multicolumn{3}{|c|}{ Age Group } & \multirow[b]{2}{*}{ Total } & \multicolumn{3}{|c|}{ Age Group } & \multirow[b]{2}{*}{ Total } & \multicolumn{3}{|c|}{ Age Group } & \multirow[b]{2}{*}{ Total } & \multicolumn{3}{|c|}{ Age Group } & \multirow[b]{2}{*}{ Total } \\
\hline & $<14$ & $\begin{array}{c}14 \text { to } \\
64\end{array}$ & $65+$ & & $<14$ & $\begin{array}{c}14 \text { to } \\
64\end{array}$ & $65+$ & & $<14$ & $\begin{array}{c}14 \text { to } \\
64\end{array}$ & $65+$ & & $<14$ & $\begin{array}{c}14 \text { to } \\
64\end{array}$ & $65+$ & \\
\hline Aug-01 & 3 & 8 & 0 & 11 & 3 & 3 & 0 & 6 & 0 & 5 & 2 & 7 & 0 & 6 & 3 & 9 \\
\hline Sep-01 & 5 & 6 & 1 & 12 & 3 & 3 & 0 & 6 & 1 & 2 & 2 & 5 & 0 & 1 & 1 & 2 \\
\hline Oct-01 & 0 & 9 & 2 & 11 & 1 & 6 & 0 & 7 & 3 & 5 & 3 & 11 & 0 & 4 & 0 & 4 \\
\hline Nov-01 & 2 & 5 & 1 & 8 & 0 & 4 & 0 & 4 & 1 & 4 & 1 & 6 & 0 & 8 & 2 & 10 \\
\hline Dec-01 & 5 & 5 & 1 & 11 & 0 & 2 & 1 & 3 & 1 & 6 & 0 & 7 & 1 & 8 & 0 & 9 \\
\hline Jan-02 & 2 & 9 & 0 & 11 & 1 & 5 & 1 & 7 & 0 & 2 & 2 & 4 & 0 & 9 & 0 & 9 \\
\hline Feb-02 & 6 & 6 & 1 & 13 & 1 & 3 & 1 & 5 & 0 & 1 & 2 & 3 & 0 & 5 & 0 & 5 \\
\hline Mar-02 & 3 & 6 & 1 & 10 & 0 & 2 & 3 & 5 & 0 & 7 & 4 & 11 & 1 & 6 & 1 & 8 \\
\hline Apr-02 & 3 & 5 & 1 & 9 & 0 & 3 & 0 & 3 & 1 & 1 & 1 & 3 & 0 & 4 & 0 & 4 \\
\hline May-02 & 2 & 2 & 0 & 4 & 2 & 4 & 0 & 6 & 1 & 5 & 1 & 7 & 0 & 8 & 4 & 12 \\
\hline Jun-02 & 1 & 2 & 0 & 3 & 2 & 3 & 1 & 6 & 0 & 5 & 2 & 7 & 0 & 3 & 0 & 3 \\
\hline Jul-02 & 0 & 12 & 0 & 12 & 0 & 3 & 1 & 4 & 0 & 3 & 0 & 3 & 0 & 2 & 2 & 4 \\
\hline Aug-02 & 4 & 2 & 1 & 7 & 1 & 5 & 0 & 6 & 0 & 1 & 3 & 4 & 0 & 6 & 1 & 7 \\
\hline Sep-02 & 4 & 5 & 0 & 9 & 0 & 2 & 0 & 2 & 0 & 4 & 2 & 6 & 0 & 5 & 2 & 7 \\
\hline Oct-02 & 3 & 7 & 0 & 10 & 2 & 5 & 0 & 7 & 1 & 4 & 0 & 5 & 0 & 6 & 2 & 8 \\
\hline Nov-02 & 2 & 7 & 0 & 9 & 1 & 7 & 0 & 8 & 2 & 6 & 1 & 9 & 0 & 9 & 1 & 10 \\
\hline Dec-02 & 2 & 6 & 1 & 9 & 0 & 1 & 1 & 2 & 0 & 6 & 2 & 8 & 0 & 5 & 4 & 9 \\
\hline Jan-03 & 2 & 4 & 0 & 6 & 2 & 7 & 0 & 9 & 1 & 2 & 0 & 3 & 0 & 3 & 1 & 4 \\
\hline Feb-03 & 2 & 3 & 0 & 5 & 1 & 1 & 0 & 2 & 0 & 6 & 3 & 9 & 1 & 6 & 0 & 7 \\
\hline Mar-03 & 1 & 5 & 0 & 6 & 1 & 2 & 0 & 3 & 0 & 4 & 2 & 6 & 0 & 7 & 1 & 8 \\
\hline Apr-03 & 3 & 4 & 0 & 7 & 0 & 3 & 0 & 3 & 0 & 3 & 3 & 6 & 0 & 3 & 0 & 3 \\
\hline May-03 & 1 & 2 & 0 & 3 & 0 & 1 & 0 & 1 & 1 & 7 & 2 & 10 & 0 & 3 & 2 & 5 \\
\hline Jun-03 & 0 & 9 & 1 & 10 & 0 & 4 & 0 & 4 & 0 & 4 & 1 & 5 & 1 & 4 & 1 & 6 \\
\hline Jul-03 & 3 & 6 & 0 & 9 & 0 & 0 & 0 & 0 & 1 & 4 & 2 & 7 & 0 & 3 & 0 & 3 \\
\hline Aug-03 & 2 & 3 & 1 & 6 & 1 & 6 & 0 & 7 & 1 & 2 & 1 & 4 & 0 & 6 & 2 & 8 \\
\hline Sep-03 & 1 & 4 & 0 & 5 & 1 & 2 & 0 & 3 & 0 & 4 & 2 & 6 & 0 & 5 & 2 & 7 \\
\hline Oct-03 & 3 & 2 & 0 & 5 & 1 & 1 & 0 & 2 & 0 & 5 & 5 & 10 & 0 & 9 & 3 & 12 \\
\hline Nov-03 & 1 & 5 & 0 & 6 & 0 & 3 & 2 & 5 & 0 & 4 & 4 & 8 & 0 & 1 & 1 & 2 \\
\hline Dec-03 & 3 & 1 & 0 & 4 & 1 & 1 & 0 & 2 & 0 & 4 & 4 & 8 & 1 & 3 & 3 & 7 \\
\hline Jan-04 & 2 & 7 & 0 & 9 & 3 & 5 & 0 & 8 & 0 & 4 & 4 & 8 & 0 & 8 & 4 & 12 \\
\hline Feb-04 & 5 & 4 & 1 & 10 & 0 & 2 & 0 & 2 & 1 & 6 & 2 & 9 & 0 & 1 & 0 & 1 \\
\hline Mar-04 & 1 & 9 & 0 & 10 & 2 & 2 & 1 & 5 & 2 & 5 & 3 & 10 & 0 & 6 & 1 & 7 \\
\hline Apr-04 & 1 & 7 & 2 & 10 & 2 & 4 & 0 & 6 & 0 & 3 & 3 & 6 & 0 & 4 & 1 & 5 \\
\hline May-04 & 1 & 10 & 0 & 11 & 1 & 1 & 0 & 2 & 0 & 5 & 1 & 6 & 0 & 6 & 2 & 8 \\
\hline Jun-04 & 1 & 4 & 2 & 7 & 0 & 0 & 0 & 0 & 0 & 5 & 1 & 6 & 0 & 6 & 1 & 7 \\
\hline
\end{tabular}




\begin{tabular}{|c|c|c|c|c|c|c|c|c|c|c|c|c|c|c|c|c|}
\hline \multirow[b]{3}{*}{ Date } & \multicolumn{4}{|c|}{ Liberty City } & \multicolumn{4}{|c|}{ Little Haiti } & \multicolumn{4}{|c|}{ Little Havana } & \multicolumn{4}{|c|}{ South Beach } \\
\hline & \multicolumn{3}{|c|}{ Age Group } & \multirow[b]{2}{*}{ Total } & \multicolumn{3}{|c|}{ Age Group } & \multirow[b]{2}{*}{ Total } & \multicolumn{3}{|c|}{ Age Group } & \multirow[b]{2}{*}{ Total } & \multicolumn{3}{|c|}{ Age Group } & \multirow[b]{2}{*}{ Total } \\
\hline & $<14$ & $\begin{array}{c}14 \text { to } \\
64\end{array}$ & $65+$ & & $<14$ & $\begin{array}{c}14 \text { to } \\
64 \\
\end{array}$ & $65+$ & & $<14$ & $\begin{array}{c}14 \text { to } \\
64\end{array}$ & $65+$ & & $<14$ & $\begin{array}{c}14 \text { to } \\
64\end{array}$ & $65+$ & \\
\hline Jul-04 & 2 & 4 & 0 & 6 & 0 & 5 & 1 & 6 & 1 & 2 & 1 & 4 & 0 & 5 & 2 & 7 \\
\hline Aug-04 & 2 & 2 & 1 & 5 & 0 & 6 & 0 & 6 & 0 & 5 & 1 & 6 & 0 & 4 & 0 & 4 \\
\hline Sep-04 & 1 & 1 & 1 & 3 & 0 & 0 & 0 & 0 & 0 & 2 & 1 & 3 & 0 & 0 & 0 & 0 \\
\hline Oct-04 & 2 & 7 & 1 & 10 & 2 & 4 & 1 & 7 & 1 & 3 & 2 & 6 & 0 & 4 & 1 & 5 \\
\hline Nov-04 & 1 & 3 & 0 & 4 & 2 & 2 & 0 & 4 & 0 & 3 & 6 & 9 & 0 & 4 & 1 & 5 \\
\hline Dec-04 & 3 & 5 & 0 & 8 & 0 & 5 & 0 & 5 & 1 & 2 & 2 & 5 & 0 & 8 & 1 & 9 \\
\hline Subtotal & 285 & 602 & 65 & 952 & 124 & 305 & 42 & 471 & 65 & 400 & 199 & 664 & 25 & 625 & 146 & 796 \\
\hline Missing & & & & 59 & & & & 42 & & & & 59 & & & & 35 \\
\hline $\begin{array}{l}\text { Total Miss } \\
\text { Total Cras }\end{array}$ & $\begin{array}{l}195 \\
3,078\end{array}$ & & & & & & & & & & & & & & & \\
\hline
\end{tabular}




\section{APPENDIX D: CRASH TYPES EFFECTS}

\section{Liberty City}

In the Liberty City neighborhood, dash/dart-out crashes accounted for the largest share of pedestrian-motor vehicle collisions over the nine years, closely followed by pedestrian crossing roadway, and vehicle not turning (Table 35). The latter crash group involves situations in which pedestrians are crossing the roadway and failed to yield at a midblock or intersection location as well as those in which the motorist failed to yield to pedestrians crossing at these locations. The top seven types of crashes accounted for nearly three-fourths of the pedestrian-involved collisions. A sizable proportion, $18 \%$, lacked sufficient details about the crashes to be classified more specifically and are thought to be difficult to address with specific countermeasures (other, unknown $=18 \%$ ). Unusual circumstances collisions, which accounted for $8 \%$ of pedestrian collisions, are also ones that are difficult to target with specific countermeasures.

Children (age 1 to 13$)$ were over-represented in dash/dart-out crashes $(31.5 \%$ of child crashes over all years compared with $17.1 \%$ for all ages [data not shown]). Children were also somewhat over-represented in unique midblock crashes (4.4\% compared with $1.9 \%$ for all ages), a type that includes going to or from a mailbox, a vendor truck, or entering or exiting parked vehicles; and in working/playing in roadway (4.8\% of child pedestrian crashes compared with $2.7 \%$ for all ages). Children had low involvement in crossing roadway - vehicle turning and were also underrepresented in crossing roadway - vehicle not turning type crashes.

In particular, both number and proportion of child dash/dart-out crashes declined over the study period. Children were involved in an average of 11.2 dash/dart crashes in the before years (1996 -2001 ) of the study period representing 34\% of all child crashes, and an average of 6 such crashes in the three after years representing $25 \%$ of all child crashes for this period $(2002-$ 2004), based on data for which both crash type and age of pedestrian were not missing. (Crash type information was missing for $6.1 \%$ of before years and $6.8 \%$ of after years cases, but it is not thought that this similar proportion of missing cases contributes to any systematic bias in the results discussed above.) Children also demonstrated declining involvement in both unique midblock and working/playing in roadway crashes. Other/unknown crash types also exhibited sizable declines for children, from an average of 5.8 crashes per year in the before years to 2.3 crashes per year in the after years, even though children were not over-represented in these nonspecific types. The decline in other/unknown could possibly be associated with a change in crash typing over the study period that resulted in fewer crashes being assigned to these indefinite types. If real, however, this is good news, since these non-specific types of crashes have not been considered to be very amenable to specific countermeasures. No decline was noted in crossing roadway - vehicle not turning crashes, another crash type that comprised above $10 \%$ of child crashes, but which was less than the proportion for the general population.

The adult cohort 14 to 64 years old demonstrated only slight over-representation in various types of crashes and was under-represented in dash/dart-out crashes as were older adults (data not shown). Those 65 and older were over-involved in crossing roadway - vehicle not turning (32.3\% compared with $17.4 \%$ over all) and in crossing roadway - vehicle turning types of 
crashes $(24.2 \%$ older adult crashes compared with $10.2 \%$ over all ages). Older adults exhibited declines in the yearly frequency of both these crash types, from the before to the after period.

Table 35. Liberty City Overall Crash Group Frequencies and Percentages by Year.

\begin{tabular}{|c|c|c|c|c|c|c|c|c|c|c|}
\hline Crash Group & 1996 & 1997 & 1998 & 1999 & 2000 & 2001 & 2002 & 2003 & 2004 & Total \\
\hline \multirow{2}{*}{ Dash/Dart-Out } & 30 & 31 & 24 & 16 & 18 & 17 & 14 & 6 & 13 & 169 \\
\hline & 22.9 & 24.2 & 20.5 & 15.5 & 17.3 & 16.2 & 13.1 & 8.3 & 16.3 & 17.9 \\
\hline \multirow{2}{*}{$\begin{array}{l}\text { Crossing Roadway } \\
\text { - Vehicle Not } \\
\text { Turning }\end{array}$} & 15 & 15 & 16 & 13 & 32 & 18 & 21 & 18 & 12 & 160 \\
\hline & 11.5 & 11.7 & 13.7 & 12.6 & 30.8 & 17.1 & 19.6 & 25.0 & 15.0 & 16.9 \\
\hline \multirow{2}{*}{$\begin{array}{l}\text { Crossing Roadway } \\
\text { - Vehicle Turning } \\
\end{array}$} & 9 & 13 & 12 & 10 & 13 & 13 & 15 & 6 & 4 & 95 \\
\hline & 6.9 & 10.2 & 10.3 & 9.7 & 12.5 & 12.4 & 14.0 & 8.3 & 5.0 & 10.0 \\
\hline \multirow{2}{*}{\begin{tabular}{|l|} 
Unusual \\
Circumstances \\
\end{tabular}} & 9 & 7 & 6 & 4 & 8 & 12 & 10 & 7 & 10 & 73 \\
\hline & 6.9 & 5.5 & 5.1 & 3.9 & 7.7 & 11.4 & 9.4 & 9.7 & 12.5 & 7.7 \\
\hline \multirow{2}{*}{ Off Roadway } & 7 & 9 & 9 & 6 & 6 & 7 & 7 & 8 & 8 & 67 \\
\hline & 5.3 & 7.0 & 7.7 & 5.8 & 5.8 & 6.7 & 6.5 & 11.1 & 10.0 & 7.1 \\
\hline \multirow{2}{*}{$\begin{array}{l}\text { Pedestrian in } \\
\text { Roadway }\end{array}$} & 5 & 2 & 11 & 7 & 0 & 4 & 2 & 19 & 16 & 66 \\
\hline & 3.8 & 1.6 & 9.4 & 6.8 & 0.0 & 3.8 & 1.9 & 26.4 & 20.0 & 7.0 \\
\hline \multirow{2}{*}{ Backing Vehicle } & 6 & 10 & 6 & 2 & 6 & 8 & 9 & 1 & 2 & 50 \\
\hline & 4.6 & 7.8 & 5.1 & 1.9 & 5.8 & 7.6 & 8.4 & 1.4 & 2.5 & 5.3 \\
\hline \multirow{2}{*}{$\begin{array}{l}\text { Walking Along } \\
\text { Roadway }\end{array}$} & 5 & 1 & 3 & 5 & 4 & 2 & 3 & 2 & 3 & 28 \\
\hline & 3.8 & 0.8 & 2.6 & 4.9 & 3.9 & 1.9 & 2.8 & 2.8 & 3.8 & 3.0 \\
\hline \multirow{2}{*}{$\begin{array}{l}\text { Working/Playing in } \\
\text { Roadway }\end{array}$} & 3 & 5 & 3 & 2 & 2 & 5 & 1 & 3 & 1 & 25 \\
\hline & 2.3 & 3.9 & 2.6 & 1.9 & 1.9 & 4.8 & 0.9 & 4.2 & 1.3 & 2.6 \\
\hline \multirow{2}{*}{$\begin{array}{l}\text { Unique } \\
\text { Midblock }\end{array}$} & 1 & 1 & 5 & 3 & 3 & 0 & 2 & 0 & 2 & 17 \\
\hline & 0.8 & 0.8 & 4.3 & 2.9 & 2.9 & 0.0 & 1.9 & 0.0 & 2.5 & 1.8 \\
\hline \multirow{2}{*}{$\begin{array}{l}\begin{array}{l}\text { Multiple Threat/ } \\
\text { Trapped }\end{array} \\
\end{array}$} & 1 & 1 & 0 & 3 & 2 & 2 & 2 & 0 & 0 & 11 \\
\hline & 0.8 & 0.8 & 0.0 & 2.9 & 1.9 & 1.9 & 1.9 & 0.0 & 0.0 & 1.2 \\
\hline \multirow{2}{*}{ Bus-Related } & 2 & 1 & 1 & 0 & 0 & 2 & 1 & 0 & 1 & 8 \\
\hline & 1.5 & 0.8 & 0.9 & 0.0 & 0.0 & 1.9 & 0.9 & 0.0 & 1.3 & 0.8 \\
\hline \multirow{2}{*}{$\begin{array}{l}\text { Crossing } \\
\text { Driveway/Alley }\end{array}$} & 1 & 1 & 1 & 0 & 1 & 0 & 1 & 0 & 0 & 5 \\
\hline & 0.8 & 0.8 & 0.9 & 0.0 & 1.0 & 0.0 & 0.9 & 0.0 & 0.0 & 0.5 \\
\hline \multirow{2}{*}{\begin{tabular}{|l|} 
Crossing \\
Expressway \\
\end{tabular}} & 0 & 0 & 1 & 0 & 0 & 1 & 1 & 0 & 1 & 4 \\
\hline & 0.0 & 0.0 & 0.9 & 0.0 & 0.0 & 1.0 & 0.9 & 0.0 & 1.3 & 0.4 \\
\hline \multirow{2}{*}{ Waiting to Cross } & 0 & 0 & 0 & 0 & 0 & 0 & 0 & 1 & 0 & 1 \\
\hline & 0.0 & 0.0 & 0.0 & 0.0 & 0.0 & 0.0 & 0.0 & 1.4 & 0.0 & 0.1 \\
\hline \multirow{2}{*}{ Other, Unknown } & 37 & 31 & 19 & 32 & 9 & 14 & 18 & 1 & 7 & 168 \\
\hline & 28.2 & 24.2 & 16.2 & 31.1 & 8.7 & 13.3 & 16.8 & 1.4 & 8.8 & 17.7 \\
\hline Total* & 131 & 128 & 117 & 103 & 104 & 105 & 107 & 72 & 80 & $947 *$ \\
\hline
\end{tabular}

Refer to Appendix A for a list of crash type codes.

\section{Little Haiti}

Pedestrian crossing the roadway and being struck by vehicle not turning accounted for the largest share $(>16 \%)$ of collisions with pedestrians in Little Haiti (Table 36). Dash/dart-out crashes accounted for $12 \%$ with unusual circumstances, crossing roadway - vehicle turning, and backing vehicle crash types each accounting for 8 to $9 \%$. With $6 \%$ off-roadway crashes, the top six types of crashes accounted for over $60 \%$ of pedestrian-motor vehicle collisions in this area. Again, a 
sizable number, about one-fifth of crashes could not be assigned a specific crash type (other/unknown). It is more difficult to detect definite downward trends among the year-to-year fluctuations in crash types, similar to the data over all in Little Haiti. There appears, however, to be a decline in dash/dart-outs from 2002 to 2003 and 2004.

Again, children accounted for over half of the dash/dart-out crashes; almost a fourth of pedestrian crashes involving children younger than 14 in Little Haiti were this type. Children were again over-represented in unique midblock, and in bus-related crashes in this community. Bus-related crashes accounted for less than $2 \%$ of pedestrian crashes in the community, but did not show a decline over the study period. In the before period, children were involved in an average of 3.3 dash/dart-out crashes per year, and in the after period, $2.7 \%$. child involvement declines were seen in unique midblock, from an average of 2 crashes per year to 0.7 per year; and in the other crash types in which children had substantial involvement (more than 10 crashes) including crossing roadway - vehicle not turning, from 2 per year to 1.3; and in the nonspecific other/unknown crashes, from 2.2 to an average 1 crash per year.

Again, patterns are less obvious among adults (14 to 64), perhaps because this group comprised such a large age range and the majority of crashes. Adults tended to be slightly over-involved in backing vehicle crashes (which in most studies tends to involve older and younger ages more frequently), in other/unknown types of crashes, and in crossing roadway - vehicle not turning crashes, but perhaps only because younger and older ages tended to be less involved in these types. There were no notable declines in adult crashes except in the other/unknown category.

There was relatively low crash involvement among older adults in general in this neighborhood. Older adults were, however, over-represented in unusual circumstance crashes, in pedestrian in roadway crashes, and were slightly over-involved in crossing roadway - vehicle not turning crashes. There may be behavioral differences among the different communities since the pedestrian in roadway results suggest that older adults in this community are perhaps more often standing, walking, or even lying in the roadway when struck (as opposed to clearly crossing or walking along the roadway). These percentages are based on only 39 crashes among older pedestrians in total, however, so no obvious conclusions should be reached. As with overall crashes, no declines were seen in the rate of pedestrian crashes among older adults in these types. 
Table 36. Little Haiti Overall Crash Group Frequencies and Percentages by Year.

\begin{tabular}{|c|c|c|c|c|c|c|c|c|c|c|}
\hline Crash Group & 1996 & 1997 & 1998 & 1999 & 2000 & 2001 & 2002 & 2003 & 2004 & Total \\
\hline \multirow{2}{*}{$\begin{array}{l}\text { Crossing Roadway - } \\
\text { Veh. Not Turning }\end{array}$} & 9 & 3 & 6 & 7 & 10 & 11 & 13 & 9 & 10 & 78 \\
\hline & 14.8 & 6.3 & 11.3 & 15.2 & 18.9 & 22.9 & 18.8 & 19.2 & 20.0 & 16.4 \\
\hline \multirow{2}{*}{ Dash/Dart-Out } & 7 & 6 & 8 & 7 & 4 & 7 & 11 & 3 & 4 & 57 \\
\hline & 11.5 & 12.5 & 15.1 & 15.2 & 7.6 & 14.6 & 15.9 & 6.4 & 8.0 & 12.0 \\
\hline \multirow{2}{*}{$\begin{array}{l}\text { Unusual } \\
\text { Circumstances }\end{array}$} & 2 & 4 & 2 & 1 & 10 & 4 & 11 & 4 & 5 & 43 \\
\hline & 3.3 & 8.3 & 3.8 & 2.2 & 18.9 & 8.3 & 15.9 & 8.5 & 10.0 & 9.1 \\
\hline \multirow{2}{*}{$\begin{array}{l}\text { Crossing Roadway - } \\
\text { Vehicle Turning }\end{array}$} & 7 & 2 & 3 & 5 & 4 & 6 & 6 & 3 & 6 & 42 \\
\hline & 11.5 & 4.2 & 5.7 & 10.9 & 7.6 & 12.5 & 8.7 & 6.4 & 12.0 & 8.8 \\
\hline \multirow{2}{*}{ Backing Vehicle } & 2 & 3 & 2 & 3 & 3 & 4 & 7 & 7 & 8 & 39 \\
\hline & 3.3 & 6.3 & 3.8 & 6.6 & 5.7 & 8.3 & 10.1 & 14.9 & 16.0 & 8.2 \\
\hline \multirow{2}{*}{ Off Roadway } & 3 & 3 & 4 & 4 & 5 & 2 & 4 & 4 & 0 & 29 \\
\hline & 4.9 & 6.3 & 7.6 & 8.7 & 9.4 & 4.2 & 5.8 & 8.5 & 0 & 6.1 \\
\hline \multirow{2}{*}{$\begin{array}{l}\text { Working/Playing in } \\
\text { Roadway }\end{array}$} & 2 & 1 & 0 & 0 & 1 & 0 & 0 & 0 & 2 & 6 \\
\hline & 3.3 & 2.1 & 0 & 0 & 1.9 & 0.0 & 0.0 & 0.0 & 4.0 & 1.3 \\
\hline \multirow{2}{*}{$\begin{array}{l}\text { Pedestrian in } \\
\text { Roadway }\end{array}$} & 0 & 2 & 3 & 4 & 1 & 1 & 1 & 8 & 4 & 24 \\
\hline & 0 & 4.2 & 5.7 & 8.7 & 1.9 & 2.1 & 1.5 & 17.0 & 8.0 & 5.1 \\
\hline \multirow{2}{*}{$\begin{array}{l}\text { Walking Along } \\
\text { Roadway }\end{array}$} & 1 & 0 & 4 & 3 & 1 & 2 & 1 & 3 & 4 & 19 \\
\hline & 1.6 & 0.0 & 7.6 & 6.5 & 1.9 & 4.2 & 1.5 & 6.4 & 8.0 & 4.0 \\
\hline \multirow{2}{*}{ Unique Midblock } & 4 & 3 & 1 & 1 & 4 & 1 & 0 & 0 & 2 & 16 \\
\hline & 6.6 & 6.3 & 1.9 & 2.2 & 7.6 & 2.0 & 0.0 & 0.0 & 4.0 & 3.4 \\
\hline \multirow{2}{*}{ Bus-Related } & 0 & 1 & 1 & 0 & 1 & 1 & 1 & 1 & 2 & 8 \\
\hline & 0.0 & 2.1 & 1.9 & 0.0 & 1.9 & 2.1 & 1.5 & 2.1 & 4.0 & 1.7 \\
\hline \multirow{2}{*}{$\begin{array}{l}\text { Multiple Threat/ } \\
\text { Trapped }\end{array}$} & 1 & 2 & 1 & 1 & 0 & 2 & 0 & 1 & 0 & 8 \\
\hline & 1.6 & 4.2 & 1.9 & 2.2 & 0.0 & 4.2 & 0.0 & 2.1 & 0.0 & 1.7 \\
\hline \multirow{2}{*}{$\begin{array}{l}\text { Crossing } \\
\text { Driveway/Alley }\end{array}$} & 0 & 1 & 1 & 0 & 0 & 0 & 1 & 0 & 1 & 4 \\
\hline & 0.0 & 2.1 & 1.9 & 0.0 & 0.0 & 0.0 & 1.5 & 0.0 & 2.0 & 0.8 \\
\hline \multirow{2}{*}{ Waiting to Cross } & 0 & 0 & 1 & 1 & 0 & 0 & 0 & 1 & 0 & 3 \\
\hline & 0.0 & 0.0 & 1.9 & 2.2 & 0.0 & 0.0 & 0.0 & 2.1 & 0.0 & 0.6 \\
\hline \multirow{2}{*}{$\begin{array}{l}\text { Crossing } \\
\text { Expressway }\end{array}$} & 0 & 0 & 0 & 0 & 0 & 0 & 0 & 0 & 0 & 0 \\
\hline & 0.0 & 0.0 & 0.0 & 0.0 & 0.0 & 0.0 & 0.0 & 0.0 & 0.0 & 0.0 \\
\hline \multirow{2}{*}{ Other, Unknown } & 23 & 17 & 16 & 9 & 9 & 7 & 13 & 3 & 2 & 99 \\
\hline & 37.7 & 35.4 & 30.2 & 19.6 & 17.0 & 14.6 & 18.8 & 6.4 & 4.0 & 20.8 \\
\hline Total* & 61 & 48 & 53 & 46 & 53 & 48 & 69 & 47 & 50 & $475^{*}$ \\
\hline
\end{tabular}

\section{Little Havana}

The top seven types of crashes accounted for more than $75 \%$ of crashes in Little Havana; all others represented less than $2 \%$ (Table 37). Pedestrians were most often struck while crossing the roadway, with vehicles either going straight (not turning, 18.4\%) or turning $(17.2 \%)$.

Dash/dart-out crashes accounted for another $12 \%$, backing vehicle crashes $10.3 \%$, and unusual circumstances and pedestrian in roadway $7.5 \%$ and $5.5 \%$. Again, patterns in the most frequently occurring crashes are as, or more likely, to be increasing as decreasing over the study period, consistent with the lack of a significant reduction in all crashes in this community. 
Table 37. Little Havana Overall Crash Group Frequencies and Percentages by Year.

\begin{tabular}{|c|c|c|c|c|c|c|c|c|c|c|}
\hline Crash Group & 1996 & 1997 & 1998 & 1999 & 2000 & 2001 & 2002 & 2003 & 2004 & Total \\
\hline \multirow{2}{*}{$\begin{array}{l}\text { Crossing Roadway - } \\
\text { Vehicle Not Turning }\end{array}$} & 12 & 12 & 11 & 7 & 21 & 16 & 17 & 15 & 12 & 123 \\
\hline & 14.3 & 20.7 & 14.1 & 13.0 & 27.3 & 20.3 & 23.0 & 17.7 & 15.0 & 18.4 \\
\hline \multirow{2}{*}{$\begin{array}{l}\text { Crossing Roadway - } \\
\text { Vehicle Turning }\end{array}$} & 15 & 8 & 14 & 11 & 4 & 7 & 10 & 27 & 19 & 115 \\
\hline & 17.9 & 13.8 & 18.0 & 20.4 & 5.2 & 8.9 & 13.5 & 31.8 & 23.8 & 17.2 \\
\hline \multirow{2}{*}{ Dash/Dart Out } & 11 & 8 & 12 & 10 & 11 & 10 & 6 & 4 & 8 & 80 \\
\hline & 13.1 & 13.8 & 15.4 & 18.5 & 14.3 & 12.7 & 8.1 & 4.7 & 10.0 & 12.0 \\
\hline \multirow{2}{*}{ Backing Vehicle } & 10 & 7 & 7 & 7 & 6 & 10 & 7 & 5 & 10 & 69 \\
\hline & 11.9 & 12.1 & 9.0 & 13.0 & 7.8 & 12.7 & 9.5 & 5.9 & 12.5 & 10.3 \\
\hline \multirow{2}{*}{$\begin{array}{l}\text { Unusual } \\
\text { Circumstances }\end{array}$} & 3 & 6 & 6 & 6 & 8 & 2 & 4 & 7 & 8 & 50 \\
\hline & 3.6 & 10.3 & 7.7 & 11.1 & 10.4 & 2.5 & 5.4 & 8.2 & 10.0 & 7.5 \\
\hline \multirow{2}{*}{$\begin{array}{l}\text { Pedestrian in } \\
\text { Roadway }\end{array}$} & 1 & 4 & 2 & 2 & 4 & 1 & 1 & 15 & 7 & 37 \\
\hline & 1.2 & 6.9 & 2.6 & 3.7 & 5.2 & 1.3 & 1.4 & 17.7 & 8.8 & 5.5 \\
\hline \multirow{2}{*}{ Off Roadway } & 2 & 4 & 2 & 1 & 4 & 5 & 2 & 4 & 6 & 30 \\
\hline & 2.4 & 6.9 & 2.6 & 1.9 & 5.2 & 6.3 & 2.7 & 4.7 & 7.5 & 4.5 \\
\hline \multirow{2}{*}{$\begin{array}{l}\text { Walking Along } \\
\text { Roadway }\end{array}$} & 5 & 0 & 0 & 0 & 2 & 3 & 0 & 2 & 1 & 13 \\
\hline & 5.6 & 0.0 & 0.0 & 0.0 & 2.6 & 3.8 & 0.0 & 2.4 & 1.3 & 1.9 \\
\hline \multirow{2}{*}{$\begin{array}{l}\text { Working/Playing in } \\
\text { Roadway }\end{array}$} & 1 & 0 & 2 & 1 & 3 & 2 & 1 & 0 & 0 & 10 \\
\hline & 1.2 & 0.0 & 2.6 & 1.9 & 3.9 & 2.5 & 1.4 & 0.0 & 0.0 & 1.5 \\
\hline \multirow{2}{*}{$\begin{array}{l}\text { Multiple Threat/ } \\
\text { Trapped }\end{array}$} & 2 & 1 & 1 & 0 & 0 & 2 & 1 & 1 & 1 & 9 \\
\hline & 2.4 & 1.7 & 1.3 & 0.0 & 0.0 & 2.5 & 1.4 & 1.2 & 1.3 & 1.4 \\
\hline \multirow{2}{*}{$\begin{array}{l}\text { Crossing } \\
\text { Driveway/Alley }\end{array}$} & 0 & 0 & 3 & 1 & 2 & 0 & 2 & 0 & 1 & 9 \\
\hline & 0.0 & 0.0 & 3.9 & 1.9 & 2.6 & 0.0 & 2.7 & 0.0 & 1.3 & 1.4 \\
\hline \multirow{2}{*}{ Unique Midblock } & 1 & 0 & 0 & 1 & 1 & 0 & 1 & 2 & 1 & 7 \\
\hline & 1.2 & 0.0 & 0.0 & 1.9 & 1.3 & 0.0 & 1.4 & 2.4 & 1.3 & 1.1 \\
\hline \multirow{2}{*}{ Bus Related } & 1 & 0 & 0 & 0 & 0 & 0 & 1 & 0 & 1 & 3 \\
\hline & 1.2 & 0.0 & 0.0 & 0.0 & 0.0 & 0.0 & 1.4 & 0.0 & 1.3 & 0.5 \\
\hline \multirow{2}{*}{ Waiting to Cross } & 0 & 1 & 0 & 0 & 0 & 1 & 1 & 0 & 0 & 3 \\
\hline & 0.0 & 1.7 & 0.0 & 0.0 & 0.0 & 1.3 & 1.4 & 0.0 & 0.0 & 0.5 \\
\hline \multirow{2}{*}{ Crossing Expressway } & 0 & 0 & 0 & 0 & 0 & 1 & 1 & 0 & 1 & 3 \\
\hline & 0.0 & 0.0 & 0.0 & 0.0 & 0.0 & 1.3 & 1.4 & 0.0 & 1.3 & 0.5 \\
\hline \multirow{2}{*}{ Other/Unknown } & 20 & 7 & 18 & 7 & 11 & 19 & 19 & 3 & 4 & 108 \\
\hline & 23.8 & 12.1 & 23.1 & 13.0 & 14.3 & 24.1 & 25.7 & 3.5 & 5.0 & 16.1 \\
\hline Total* & 84 & 58 & 78 & 54 & 77 & 79 & 74 & 85 & 80 & $669 *$ \\
\hline
\end{tabular}

Children were again highly over-represented in dash/dart-out crashes, which accounted for $40 \%$ of child crashes in Little Havana compared with $12 \%$ of pedestrian crashes for all ages. Children were highly under-represented in the top crash types - crossing the roadway and struck by turning vehicles, and to a lesser extent crossing the roadway and struck by vehicles not turning.

Adult patterns were not pronounced while older adults tended to be somewhat over-represented in crossing roadway - vehicle not turning (25.9\% compared with $17.8 \%$ over all ages) backing vehicle crashes (14.3\% compared with $10.6 \%$ over all), and other/unknown crashes $(19.6 \%$ compared with $16.3 \%$ over all). 
As described earlier, crashes did not decline in this neighborhood for adult and older adult pedestrians, but crashes among children did decrease. Among children, the most significant trend was a decline from an average of 3.2 dash/dart-out crashes per year to an average of 1.7 crashes per year, the type in which children were most involved.

\section{South Beach}

In South Beach, the top seven crash types together accounted for nearly $80 \%$ of the crashes, with crossing roadway - vehicle turning crashes accounting for above $20 \%$. Other crossing roadway with vehicle not turning crashes accounted for $14 \%$, followed by unusual circumstance crashes $(13 \%)$, backing vehicle $(12 \%)$, dash/dart-out $(8 \%)$, and off roadway and pedestrian in roadway crashes at $5 \%$ each. Crash trends for the two most frequent crash types are unclear or even increasing from before to after periods; declines may be occurring in the next three most frequent types (Table 38). 
Table 38. South Beach Overall Crash Group Frequencies and Percentages by Year.

\begin{tabular}{|c|c|c|c|c|c|c|c|c|c|c|}
\hline Crash Group & 1996 & 1997 & 1998 & 1999 & 2000 & 2001 & 2002 & 2003 & 2004 & Total \\
\hline \multirow{2}{*}{$\begin{array}{l}\text { Crossing Roadway - } \\
\text { Vehicle Turning }\end{array}$} & 17 & 13 & 14 & 18 & 28 & 18 & 30 & 18 & 13 & 169 \\
\hline & 16.8 & 13.7 & 16.9 & 18.2 & 28.3 & 22.2 & 36.1 & 24.7 & 18.3 & 21.5 \\
\hline \multirow{2}{*}{$\begin{array}{l}\text { Crossing Roadway - } \\
\text { Vehicle Not Turning }\end{array}$} & 10 & 17 & 10 & 11 & 10 & 11 & 14 & 14 & 13 & 110 \\
\hline & 9.9 & 17.9 & 12.1 & 11.1 & 10.1 & 13.56 & 16.9 & 19.2 & 18.3 & 14.0 \\
\hline \multirow{2}{*}{$\begin{array}{l}\text { Unusual } \\
\text { Circumstances }\end{array}$} & 13 & 13 & 14 & 13 & 11 & 12 & 14 & 7 & 6 & 103 \\
\hline & 12.9 & 13.7 & 16.9 & 13.1 & 11.1 & 14.8 & 16.9 & 9.6 & 8.5 & 13.1 \\
\hline \multirow{2}{*}{ Backing Vehicle } & 13 & 10 & 13 & 14 & 12 & 11 & 11 & 3 & 8 & 95 \\
\hline & 12.9 & 10.5 & 15.7 & 14.1 & 12.1 & 13.6 & 13.3 & 4.1 & 11.3 & 12.1 \\
\hline \multirow{2}{*}{ Dash/Dart-Out } & 13 & 9 & 5 & 8 & 2 & 8 & 2 & 8 & 8 & 63 \\
\hline & 12.9 & 9.5 & 6.0 & 8.1 & 2.0 & 9.9 & 2.4 & 11.0 & 11.3 & 8.0 \\
\hline \multirow{2}{*}{ Off Roadway } & 6 & 4 & 5 & 7 & 4 & 3 & 5 & 2 & 4 & 40 \\
\hline & 5.9 & 4.2 & 6.0 & 7.1 & 4.0 & 3.7 & 6.0 & 2.7 & 5.6 & 5.1 \\
\hline \multirow{2}{*}{$\begin{array}{l}\text { Pedestrian in } \\
\text { Roadway }\end{array}$} & 3 & 3 & 5 & 1 & 9 & 1 & 0 & 10 & 8 & 40 \\
\hline & 3.0 & 3.7 & 6.0 & 1.0 & 9.1 & 1.2 & 0.0 & 13.7 & 11.3 & 5.1 \\
\hline \multirow{2}{*}{ Unique Midblock } & 2 & 4 & 1 & 1 & 4 & 0 & 1 & 2 & 1 & 16 \\
\hline & 2.0 & 4.2 & 1.2 & 1.0 & 4.0 & 0.0 & 1.2 & 2.7 & 1.4 & 2.0 \\
\hline \multirow{2}{*}{$\begin{array}{l}\text { Multiple Threat/ } \\
\text { Trapped }\end{array}$} & 2 & 2 & 0 & 3 & 0 & 1 & 1 & 3 & 1 & 13 \\
\hline & 2.0 & 2.1 & 0.0 & 3.0 & 0.0 & 1.2 & 1.2 & 4.1 & 1.4 & 1.7 \\
\hline \multirow{2}{*}{$\begin{array}{l}\text { Working/Playing in } \\
\text { Roadway }\end{array}$} & 4 & 2 & 1 & 2 & 1 & 2 & 0 & 1 & 0 & 13 \\
\hline & 4.0 & 2.1 & 1.2 & 2.0 & 1.0 & 2.5 & 0.0 & 1.4 & 0.0 & 1.7 \\
\hline \multirow{2}{*}{$\begin{array}{l}\text { Crossing } \\
\text { Driveway/Alley }\end{array}$} & 1 & 1 & 0 & 1 & 0 & 2 & 1 & 0 & 5 & 11 \\
\hline & 1.0 & 1.0 & 0.0 & 1.0 & 0.0 & 2.5 & 1.2 & 0.0 & 7.0 & 1.4 \\
\hline \multirow{2}{*}{$\begin{array}{l}\text { Walking Along } \\
\text { Roadway }\end{array}$} & 1 & 0 & 0 & 2 & 3 & 1 & 1 & 2 & 1 & 11 \\
\hline & 1.0 & 0.0 & 0.0 & 2.0 & 3.0 & 1.2 & 1.2 & 2.7 & 1.4 & 1.4 \\
\hline \multirow{2}{*}{ Bus Related } & 1 & 2 & 0 & 0 & 1 & 0 & 1 & 1 & 0 & 6 \\
\hline & 1.0 & 2.1 & 0.0 & 0.0 & 1.0 & 0.0 & 1.2 & 1.4 & 0.0 & 0.8 \\
\hline \multirow{2}{*}{ Waiting to Cross } & 0 & 1 & 0 & 0 & 0 & 0 & 0 & 0 & 2 & 3 \\
\hline & 0.0 & 1.0 & 0.0 & 0.0 & 0.0 & 0.0 & 0.0 & 0.0 & 2.8 & 0.4 \\
\hline \multirow{2}{*}{ Crossing Expressway } & 0 & 0 & 0 & 0 & 0 & 0 & 0 & 0 & 0 & 0 \\
\hline & 0.0 & 0.0 & 0.0 & 0.0 & 0.0 & 0.0 & 0.0 & 0.0 & 0.0 & 0.0 \\
\hline \multirow{2}{*}{ Other/Unknown } & 15 & 14 & 15 & 18 & 14 & 11 & 2 & 2 & 1 & 92 \\
\hline & 14.9 & 14.7 & 18.1 & 18.2 & 14.1 & 13.6 & 2.4 & 2.7 & 1.4 & 11.7 \\
\hline Total* & 101 & 95 & 83 & 99 & 99 & 81 & 83 & 73 & 71 & 785* \\
\hline
\end{tabular}

There was low crash involvement among children in the South Beach community, with only 24 typed cases clearly involving children under 14 . Of these, $42 \%$ were of the dash/dart-out types of crashes; seniors were correspondingly under-represented in this crash type.

Again, the adult ages which comprise the largest portion of all crashes are fairly representative of the crash type trends in general. Older adults were again over-represented in crossing roadway vehicle turning (37.5\% compared with $21.8 \%$ over all ages) and crossing roadway - vehicle not turning crashes (18.4\% compared with $14.2 \%$, and in backing vehicle crashes $(16.2 \%$ compared 
with $11.8 \%$ over all). Older adults were less represented in pedestrian in roadway crashes, while children had no involvement in this crash type.

Child involvement in dash/dart-out crashes decreased from an average of 1.3 crashes per year to less than 1 crash per year. The adult trends are interesting. Following the general trend, there were no declines, in fact increases, in the most frequent crash types involving roadway crossings among both adults and older adults. There were, however, decreases in the rates of "unusual" circumstances cases (an average of 11.7 per year to 8 per year), Backing Vehicle ( 8 to 5.7 per year), Other/unknown (5.7 to 1.7 per year), and off-roadway crashes (3.5 to 3 per year), types that may reflect the complex resort type environment, with significant numbers of parking lot and other off-roadway crashes and complex interactions of nighttime adult-involved crashes. 
DOT HS 810964

June 2008 